\title{
Tobacco smoke extract modulates activity and expression of monoamine oxidase and $\mu$ opioid receptor in cultured human neuroblastoma cells.
}

\author{
A Thesis \\ Submitted to Victoria University of Wellington \\ in fulfillment of the requirements for the degree of \\ Doctor of Philosophy \\ in Biomedical Science
}

By

Amy Jane Lewis

Victoria University of Wellington 
Tobacco addiction is a major public health concern and is responsible for approximately five million deaths globally each year. Although most current smokers express a desire to quit, few are successful in their attempts. Nicotine is the primary neurobiologically active component in tobacco smoke and acts through the nicotinic acetylcholine receptor (nAChR) to sustain addiction. However, nicotine replacement therapies have proven to be remarkably ineffective at helping smokers quit. This indicates that nicotine alone cannot fully account for the intense and enduring nature of tobacco addiction. Previous research has provided strong evidence that monoamine oxidase (MAO) enzymes and the endogenous opioid system may also play a role in tobacco dependence.

The present study compared and contrasted the influence of nicotine and the nonnicotine components of tobacco smoke on the enzyme activity of MAO-A and MAO-B. Gene expression of MAO and the $\mu$ opioid receptor (MOR) in SH-SY5Y human neuroblastoma and U-118 MG glioma cell lines was also investigated. Using a kynuramine-based enzymatic assay adapted and optimised for this study, the MAO inhibitory activity of tobacco-based samples were tested, including total particulate matter (TPM) extracts from a range of New Zealand tobacco products, Quest ${ }^{\circledR}$ nicotinefree cigarettes, and fluid from the RUYAN ${ }^{\circledR}$ Electronic cigarette. TPM from both standard tobacco and Quest ${ }^{\circledR}$ significantly inhibited MAO-A and MAO-B activity in vitro and in cultured cells. Differences between the types and brands of tobacco products were observed. TPM derived from loose-leaf tobacco inhibited MAO enzymes more potently than samples from manufactured cigarettes. This difference was attributed to a significantly higher tar:nicotine ratio in loose-leaf tobacco. Standard TPM and Quest ${ }^{\circledR}$ TPM also inhibited total MAO activity in SH-SY5Y cells treated for 24 hours; whereas the weak activity in U-118 MG remained unchanged. However, MAO activity was highly dependent on the cell culture conditions, with activity increasing in SH-SY5Y cells when treated with a 5-day exposure regimen. This finding was unique to the present study.

The gene expression of MAO-A, MAO-B, and MOR was examined using a qRT-PCR assay. All three genes were significantly up-regulated by standard and denicotinized TPM extracts after a 5-day treatment regimen. This finding was correlated with an increase in protein abundance for MOR, but not MAO-A or MAO-B, as assayed by Western blot. Up- 
regulation of MAO and MOR gene expression was abolished when cells were treated with TPM extracts in conjunction with the $\mathrm{nAChR}$ antagonist mecamylamine, suggesting that up-regulation of MAO and MOR genes was dependent, at least in part, on nAChR signalling. Both standard TPM and TPM from denicotinized Quest ${ }^{\circledast}$ cigarettes induced inhibition of MAO and up-regulation of MAO and MOR gene expression. This demonstrates that non-nicotine compounds within tobacco smoke can significantly influence the behaviour of cultured neuronal cells. Further research is required to fully elucidate the mechanisms behind the MAO and MOR gene response, and a better understanding of these mechanisms may provide a framework for the development of novel smoking cessation therapies. 


\section{Acknowledgements}

I would like to acknowledge with gratitude and appreciation the help, guidance, and support I have received from the following people in completing this thesis.

Dr John H. Miller, for his endless support, patience, and enthusiasm, and his invaluable help in proof-reading and checking my many drafts. Also, Dr Penny Truman for her helpful ideas, encouraging words of advice, and for seeing potential in places that I didn't think to look.

Dr Donia Macartney-Coxson and Alice Johnstone for their expert knowledge and assistance with the qRT-PCR experiments used in this thesis, and for generously providing training and the use of their laboratory. Thanks also to Danny Kay for his assistance with cell culture, and his ever-sunny disposition.

Dr Rod Lea and Michael Green for their preliminary work in developing and optimizing the MAO-A and MAO-B PCR primers used in this study.

Dr Murray Laugesen, Health New Zealand Ltd, for supplying the RUYAN e-cigarette samples tested in this study.

The academic staff in the School of Biological Sciences at Victoria University. In particular, I would like to thank Dr Bronwyn Kivell and Dr Darren Day for your helpful tips and advice on the real-time PCR experiments used in this thesis. Special thanks to Dr Bill Jordan, who offered many well-timed words of support and encouragement.

Thanks to the technical and administration staff in the School of Biological Sciences, especially Mary Murray, Sandra Taylor, and Patricia Stein, for all your hard work in ensuring I had access to the resources I needed, and for chasing down my six-monthly reports.

The many students who have passed through the $6^{\text {th }}$ floor lab, including Tim Sargeant, Anja Wilmes, Kevin Crume, and Ryan Steel, for your friendship and enthusiasm.

My deepest gratitude for the generosity and support I received from my brothers, Kerry and Casey, and the extraordinary patience and understanding from my mother and step-father.

Thanks especially to all of my friends, for your encouragement, support and understanding. To my dearest friend Dean, and Ellen, for your unfailing friendship and generosity, and for always believing I could do this! To Peter, for being so very understanding and supportive. Thanks also to Andrea, Lisa, Liz, Sarah, Sass, Kelly, Sonja, and Bruce - I promise the neglect is over now l've submitted!

This project was generously funded by research grants from the Wellington Medical Research Foundation and the VUW/ESR Post-graduate Fellowship. 


\section{Table of Contents}

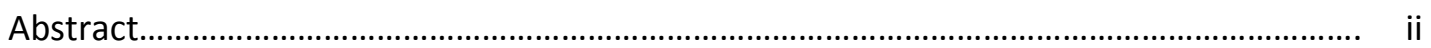

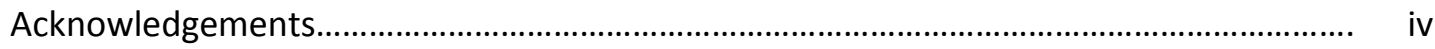

Table of Contents...................................................................................................... v

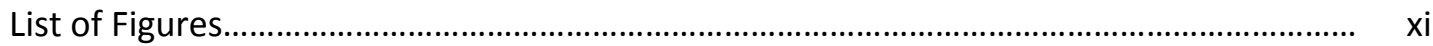

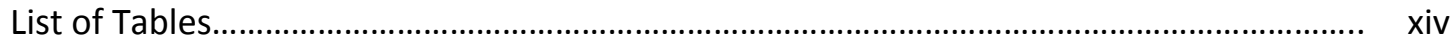

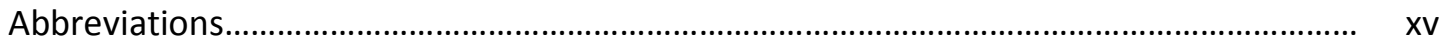

Chapter One: Literature Review 1

1.1 Biochemistry and Mode of Action of Nicotine 3

1.2 Mechanisms of Addiction 9

1.3 Introduction to Monoamine Oxidase 13

1.3.1 General Introduction 13

$\begin{array}{ll}\text { 1.3.2 Enzyme Structure } & 16\end{array}$

$\begin{array}{lll}\text { 1.3.3 Gene Structure } & 17\end{array}$

1.3.4 Localization and Activity of Monoamine Oxidase 19

1.3.4.1 Localization in the Brain 19

$\begin{array}{ll}\text { 1.3.4.2 Peripheral Localization } & 221\end{array}$

1.4 Inhibition of Monoamine Oxidase by Tobacco Smoke 23

1.4.1 MAO Inhibitors in Tobacco Smoke $\quad 27$

1.4.2 MAO Inhibitors in Smoking Cessation 32

$1.5 \quad$ Introduction to the $\boldsymbol{\mu}$ Opioid Receptor (MOR) 342

1.5.1 General Introduction 34

1.5.2 Structure, Function, and Localization 34

1.5.3 MOR and Tobacco 36

$\begin{array}{lll}1.6 & \text { Aims } & 38\end{array}$

Chapter Two: General Methods

2.1 Cell Culture $\quad 41$

2.1.1 SH-SY5Y Human Neuroblastoma $\quad 41$

2.1.2 U-118 MG Human Glioma $\quad 42$

2.1.3 Culture Conditions 43

2.1.4 Cell Passaging 43

2.2 Tobacco Particulate Exposures 44

2.2.1 Tobacco Particulate Matter Sample Collection 44

2.2.2 Cell Treatments and Exposures 45

2.3 Sample Preparation 46 
2.3.1 Protein Lysate Preparation $\quad 46$

2.3.2 TriZol $^{\circledR}$ Total RNA Extraction $\quad 47$

2.3.3 Protein Concentration Assay $\quad 47$

2.4 Monoamine Oxidase Activity Assays 47

2.4.1 Amplex ${ }^{\circledR}$ Red Monoamine Oxidase Activity Assay 48

2.4.2 Kynuramine Monoamine Oxidase Activity Assay 49

2.4.2.1 Kynuramine Microcentrifuge Tube Procedure 49

2.4.2.2 Kynuramine Microplate Procedure 50

2.5 Western Blotting $\quad 51$

2.5.1 Polyacrylamide Gel Electrophoresis $\quad 51$

2.5.2 Immunoblotting Procedure $\quad 51$

2.5.3 Antibodies $\quad 52$

2.6 Quantitative Real-Time PCR

2.6.1 mRNA Extraction $\quad 54$

2.6.2 Reverse Transcription $\quad 55$

2.6.3 Quantitative Real-Time PCR $\quad 56$

2.6.4 PCR Primer Design and Validation $\quad 57$

2.6.4.1 Agarose Gel Electrophoresis $\quad 59$

$\begin{array}{lll}2.7 & \text { Statistical Analysis } & 59\end{array}$

Chapter Three: Development of a fluorimetric MAO enzyme activity

assay 60

3.1.1 Amplex Red Assay 61

3.1.2 Kynuramine Assay $\quad 62$

3.1.3 Other MAO Assays 63

$\begin{array}{lll}3.1 .4 & \text { Objectives } & 63\end{array}$

3.2 Materials and Methods $\quad 64$

3.2.1 Sample Preparation $\quad 64$

3.2.1.1 Cultured Cell Lysates 64

3.2.1.2 Human Blood Platelets 65

32.1.3 Purified Recombinant MAO-A and MAO-B Enzymes 65

3.2.2 Invitrogen Amplex ${ }^{\circledR}$ Red Assay $\quad 65$

3.2.2.1 Using Amplex Red for Differentiated SH-SY5Y Cells 66

$\begin{array}{lll}3.2 .3 & \text { Kynuramine Assay } & 67\end{array}$

3.2.3.1 Assessing Assay Variation 67

3.2.3.2 Measuring MAO Activity in Human Blood Platelets 68

$\begin{array}{lll}3.3 & \text { Results } & 68\end{array}$

3.3.1 Amplex ${ }^{\circledR}$ Red Assay $\quad 68$

3.3.1.1 Resorufin Standard Curve 68

3.3.1.2 Using Amplex Red for Differentiated SH-SY5Y Cells 69

3.3.1.3 Using Amplex Red to Distinguish MAO-A and MAO-B activity $\quad 70$

3.3.1.4 Problems with the Amplex Red Assay 71

3.3.2 Kynuramine Assay $\quad 72$

3.3.2.1 4-Hydroxyquinoline Standard Curve 72

3.3.2.2 Determination of Optimum Substrate Concentration 73 
3.3.2.3 Fluorescence Increases Proportionally with Increased Enzyme Concentrations

3.3.2.4 Kynuramine Reaction is Linear Over Time

3.3.2.5 Kynuramine Assay Variability 75

3.3.2.6 Measuring MAO Enzyme Activity with Kynuramine 76

3.3.2.7 Measuring MAO Activity in Blood Platelets 78

$\begin{array}{lll}3.4 & \text { Discussion } & 79\end{array}$

3.4.1 Amplex Red Assay $\quad 79$

3.4.1.1 Measuring MAO Activity in Differentiated SH-SY5Y Cells $\quad 79$

3.4.1.2 Problems with the Amplex Red Assay 79

3.4.2 Kynuramine Assay $\quad 81$

3.4.2.1 Measuring MAO Activity in Human Blood Platelets 83

3.4.3 Summary 83

Chapter Four: Inhibition of MAO Enzyme Activity by Tobacco Extracts

$\begin{array}{lll}\text { 4.1.2 Objectives } & 87\end{array}$

4.2 Materials and Methods 88

4.2.1 Purified Recombinant MAO Enzymes 88

4.2.2 Kynuramine Assay $\quad 88$

4.2.3 Tobacco Extract Exposures $\quad 89$

4.2.4 Comparison of Nicotine, TPM, and Denicotinised TPM 90

4.2.5 Comparison of MAO Inhibition between Tobacco Products 91

4.2.6 RUYAN $^{\circledR}$ Cartridge Exposures 92

4.3 Results 93

4.3.1 Effect of Nicotine on MAO-A and MAO-B 93

4.3.2 Comparison of Nicotine, TPM, and Denicotinised TPM 93

4.3.3 Comparison of Nicotine Yields Between Products 94

4.3.4 Comparison of MAO Inhibition Between Tobacco Products 96

4.3.4.1 MAO Concentration-Response for TPM Samples 96

4.3.5 RUYAN ${ }^{\circledR}$ Electronic Cigarette Exposures 102

$\begin{array}{lll}4.4 & \text { Discussion } & 104\end{array}$

4.4.1 Effects of Nicotine and TPM on Monoamine Oxidase Enzymes 104

4.4.2 Comparing Nicotine and Tar Yields Between Tobacco Products 106

4.4.3 Comparing MAO Inhibition Between Tobacco Products 107

4.4.4 The RUYAN ${ }^{\circledR}$ Electronic Cigarette Does Not Inhibit MAO 109

\section{Chapter Five: MAO inhibition in cultured cells exposed to tobacco} extracts 111

5.1.1 SH-SY5Y Neuroblastoma as a Model of Human Neuronal Function 111

5.1.2 Glial Cells and Tobacco Addiction 113

$\begin{array}{lll}5.1 .3 & \text { Objectives } & 114\end{array}$

5.2 Materials and Methods 115

5.2.1 Exposure to Tobacco Particulate Matter 115

5.2.1.1 Total MAO Activity in SH-SY5Y Cells 115

5.2.1.2 Total MAO Activity in U-118 MG Cells 116

5.2.2 Kynuramine MAO Activity Assay 116

5.2.2.1 Kynuramine Microcentrifuge Tube Assay 116 
5.3 Results

5.3.1 Effects of Ethanol Exposure on Total MAO Activity in SH-SY5Y Cells 118

5.3.2 MAO Inhibition in SH-SY5Y Cells Treated with Tobacco Extracts 118

5.3.3 Tobacco Extract Exposure and Total MAO Activity in SH-SY5Y Cells Over Time

5.3.4 Effects of Tobacco Extract Exposure on Total MAO Activity in U-118 MG Cells

5.4 Discussion

5.4.1 Effects of Ethanol Exposure on Total MAO Activity in SH-SY5Y Cells 126

5.4.2 Standard and De-nicotinized Tobacco Extracts Inhibit MAO in SH-SY5Y 126

5.4.3 Changes in Total MAO Activity in SH-SY5Y Cells Exposed to Tobacco Compounds

5.4.4 Effects of Tobacco Extract Exposures on Total MAO Activity in U-118 MG Cells

5.4.5 Summary

Chapter Six: Tobacco extract exposure alters MAO gene expression

6.1.1 MAO Gene Studies and Smoking 133

6.1.2 Nicotine and Gene Expression $\quad 135$

6.1.3 Quantitative Real-Time PCR 137

6.1.3.1 Data Manipulations and Calculations $\quad 140$

$\begin{array}{lll}6.1 .4 & \text { Objectives } & 142\end{array}$

6.2 Materials and Methods $\quad 143$

6.2.1 Tobacco Particulate Matter Exposures 143

6.2.1.1 MAO Gene Expression in SH-SY5Y Cells 143

6.2.1.2 MAO Gene Expression in U-118 MG Cells 144

6.2.1.3 Gene Expression in SH-SY5Y Cells Treated with Refreshed Media 144

6.2.1.4 Mecamylamine Exposures 145

6.2.2 Quantitative Real-Time PCR 145

6.2.2.1 Reference Genes $\quad 145$

$\begin{array}{ll}\text { 6.2.2.2 Primer Efficiency } & 145\end{array}$

6.2.2.3 Data Manipulation 146

6.2.3 Western Blotting 146

$\begin{array}{llr}6.3 & \text { Results } & 147\end{array}$

6.3.1 Quantitative Real-Time PCR Validation 147

$\begin{array}{lll}\text { 6.3.1.1 Primer Efficiency } & 147\end{array}$

$\begin{array}{lll}\text { 6.3.1.2 Primer Specificity } & 148\end{array}$

6.3.1.2 Reference Genes 149

6.3.2 Effects of Ethanol Exposure on MAO-A and MAO-B Gene Expression 151

6.3.3 Effects of Tobacco Extract Exposure on MAO-A Gene Expression 152

6.3.4 Effects of Tobacco Extract Exposure on MAO-B Gene Expression 155

6.3.5 Gene Expression in U-118 MG Cells Treated with Tobacco Compounds 158

6.3.6 Mecamylamine Treatment 161

6.3.6.1 Effects of Mecamylamine 161

6.3.6.2 Validation of Mecamylamine Assay 163

6.3.6 Western Blotting 164

$\begin{array}{llr}6.4 & \text { Discussion } & 166\end{array}$ 
6.4.1 Effects of Ethanol on MAO-A and MAO-B Expression

6.4.2 Changes in MAO-A and -B Gene Expression Following Exposure to Tobacco Compounds

6.4.3 Effects of Mecamylamine Treatment on MAO-A Gene Expression 169

6.4.4 MAO-A and MAO-B Gene Expression in U-118 MG Cells 170

6.4.5 Summary

Chapter Seven: Up-regulation of MOR gene expression following tobacco exposure

7.1.2 Drugs of Abuse

7.1.3 Objectives

7.2 Materials and Methods

7.2.1 Tobacco Particulate Exposures

7.2.1.2 5-day Refreshed Medium Exposure Groups 181

7.2.1.3 Mecamylamine Exposure Groups 181

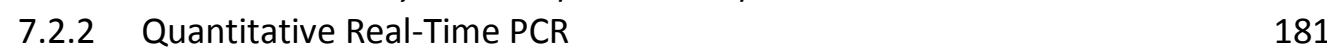

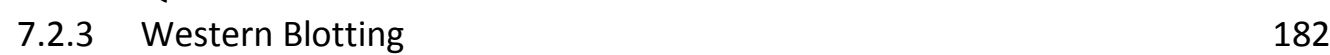

$\begin{array}{ll}7.2 .4 & \text { Data Analysis } \\ \end{array}$

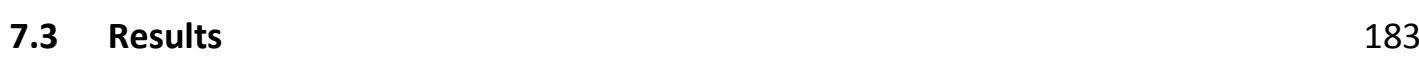

7.3.1 Validation of the qRT-PCR Assay 183

7.3.2 Effects of Ethanol Exposure on MOR Gene Expression 184

7.3.3 Effects of Tobacco Extract Exposure on MOR Gene Expression 185 7.3.3.1 Summary of Chnages in MOR Expression 187

$\begin{array}{lll}\text { 7.3.4 Effects of Mecamylamine } & 188\end{array}$

$\begin{array}{lll}\text { 7.3.5 Western Blotting } & 189\end{array}$

$\begin{array}{lll}7.4 & \text { Discussion } & 190\end{array}$

7.4.1 Effects of Ethanol on MOR Gene Expression 190

7.4.2 Tobacco Extract Exposure Increases MOR Gene Expression in SH-SY5Y 191

7.4.3 MOR Up-regulation Following Tobacco Exposure is Dependent on nAChR activation 193

$\begin{array}{lll}\text { 7.4.4 Summary } & 195\end{array}$

Chapter Eight: General Discussion and Future Directions 196

8.1 Project Summary 196

$\begin{array}{ll}\text { 8.2 Future Experiments } & 199\end{array}$

8.2.1 Investigate a Continuous Cell Culture Treatment Regimen 199

8.2.2 Identify the Neurobiologically Active Compounds in Tobacco Smoke 200

8.2.3 Elucidate the Mechanism of MAO Up-Regulation 200

8.2.4 Elucidate the Mechanism of MOR Up-Regulation 201

8.2.5 Do Tobacco Smoke Constituents Bind to or Activate MOR? 201

8.2.6 Remodel in vitro Experiments for Animal Studies 202

8.3 Towards Improved Smoking Cessation Therapies 203

$\begin{array}{ll}\text { References } & 205\end{array}$ 
Chapter Nine: Appendix

9.1 Buffers \& Solutions 220

9.2 TPM Numbering Scheme 223

9.3 RUYAN ${ }^{\circledR}$ Cartridge Ingredients List 224

9.4 PCR Primer Efficiency Data 225 


\section{Index of Figures}

\section{Chapter One}

Fig. 1(a) Nicotinic acetylcholine receptor localization in the plasma membrane................... 4

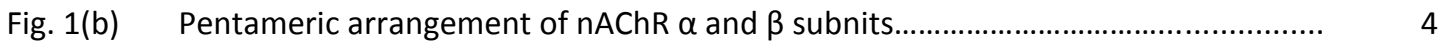

Fig. 2 The effects of nicotine binding in neuronal cells........................................................ 8

Fig. $3 \quad$ The reaction catalyzed by monoamine oxidase......................................................... 14

Fig. 4(a) Molecular schematic of monoamine oxidase A enzyme............................................ 18

Fig. 4(b) Molecular schematic of monoamine oxidase B enzyme................................................. 18

Fig. $5 \quad$ Whole body PET scan of MAO-B activity in a smoker and non-smoker..................... 24

\section{Chapter Two}

Fig. 6(a) Magnification of SH-SY5Y human neuroblastoma cells

Fig. 6(b) Magnification of U-118 MG human glioma cells

\section{Chapter Three}

Fig. 7 Resorufin standard curve

Fig. $8 \quad$ Total MAO activity in SH-SY5Y cells differentiated with retinoic acid.......................... 69

Fig. 9(a) Inhibition of MAO subtypes in SH-SY5Y cells by Amplex Red inhibitors...................... 70

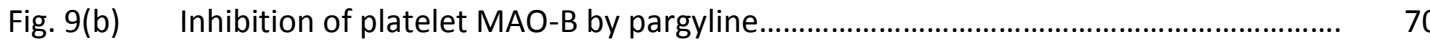

Fig. $10 \quad$ MAO activity measured in SH-SY5Y cell lysate by Amplex Red assay........................... 71

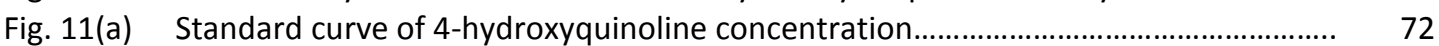

Fig. 11(b) Standard curve of 4-hydroxyquinoline concentration................................................... 72

Fig. 12(a) MAO-A activity versus substrate concentration for purified MAO-A enzyme............ 73

Fig. 12(b) MAO-A activity versus substrate concentration for SH-SY5Y cell lysate..................... 73

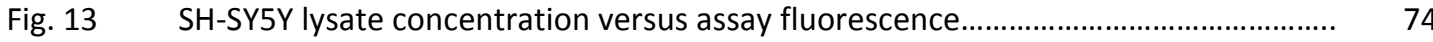

Fig. $14 \quad$ Time course of MAO-A activity measured by kynuramine assay................................ $\quad 75$

Fig. 15(a) Between sample variation for untreated SH-SY5Y cell lysates........................................ 76

Fig. 15(b) Total MAO activity in SH-SY5Y cell lysates measured on consecutive days................. 76

Fig. 16(a) Inhibition of MAO-A by clorgyline.................................................................................. 77

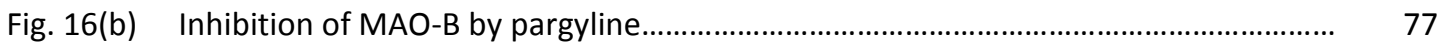

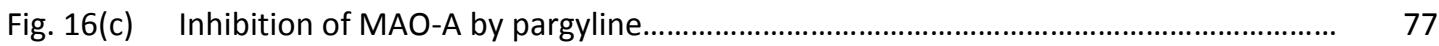

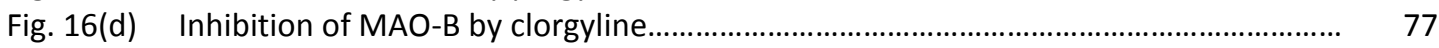

Fig. 17 Total platelet MAO-B activity in human smokers and non-smokers............................ 78

\section{Chapter Four}

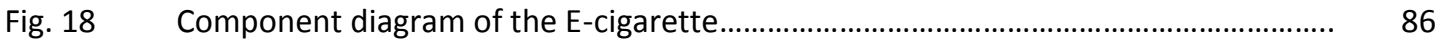

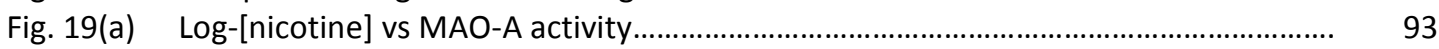

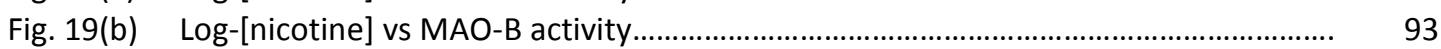

Fig. 20(a) MAO-A activity following treatment with tobacco extracts......................................... 94

Fig. 20(b) MAO-B activity following treatment with tobacco extracts....................................... 94

Fig. 21(a) Nicotine yield per cigarette for TPM derived from manufactured cigarettes and hand-rolled tobacco cigarettes.

Fig. 21(b) Tar yield for TPM derived from manufactured cigarettes and hand-rolled cigarettes.

Fig. 22(a) Comparison of dose:response curves for TPM samples derived from standard and denicotinized cigarettes for MAO-A...

Fig. 22(b) Comparison of dose:response curves for TPM samples derived from cigarettes and 
loose-leaf tobacco for MAO-A.

Fig. 23 MAO-A enzyme activity following exposure to TPM extracts ( $4 \mu \mathrm{M}$ nicotine).............

Fig. 24 MAO-A inhibition following exposure to TPM samples normalized to $0.01 \mathrm{mg} / \mathrm{mL}$ tar...

Fig. 25

Fig. 26(a)

MAO-A enzyme activity following exposure to TPM extracts $(0.1 \mathrm{mg} / \mathrm{mL}$ tar $)$...

Fig. 26(b)

MAO-A activity following 15 mins exposure to RUYAN samples.

MAO-A activity following 15 mins exposure to RUYAN samples....

Fig. 27(b) MAO-B activity following 60 mins exposure to RUYAN samples.

\section{Chapter Five}

Fig. 28 Total MAO activity in SH-SY5Y cells treated with ethanol......

Fig. 29(a) Total MAO activity Vs. substrate concentration in SH-SY5Y cells treated for 3 days..

Fig. 29(b) Total MAO activity Vs. substrate concentration in SH-SY5Y cells treated for 5 days..

Fig. 30(a) Lineweaver-Burke plot of MAO activity in SH-SY5Y cells treated with tobacco extracts for 5 days.

\section{Chapter Six}

Fig. 38(a)

Fig. 38(b)

Fig. 39

Fig. 40(a)

Fig. 40(b)

Fig. 40(c)

Fig. 41(a)

Fig. 41(b)

Fig. 42(a)

Fig. 42(b)

Fig. 43

Fig. 44(a)

Fig. 44(b)

Fig. 44(c)

Fig. 44(d)

Fig. 45

Fig. 46
SH-SY5Y dilution sequence for POLR2F RT-PCR primers

POLR2F primer amplification efficiency plot.....

Representative RT-PCR dissociation curves for POLR2F, MAO-A, and MAO-B..

Electrophoretic bands of MAO-A PCR products.

Electrophoretic bands of MAO-B PCR products.

Electrophoretic bands of POLR2F PCR products.

Comparison of reference gene expression in $\mathrm{SH}-\mathrm{SY} 5 \mathrm{Y}$ cells

Changes in MAO-A expression in SH-SY5Y following treatment with tobacco compounds for 5 days continuously. regimen. regimen. 
Fig. 47(a) MAO-B expression following treatment with nicotine by the refreshed regimen......

Fig. 47(b) MAO-B expression following treatment with HTPM by the refreshed regimen.........

Fig. 47(c) MAO-B expression following treatment with QTPM by the refreshed regimen..........

Fig. 47(d) Summary of changes in MAO-B expression following treatment with the refreshed regimen.

Fig. 48 Comparison of changes in MAO-B expression in cells treated continuously or by the refreshed regimen.

Fig. 49(a) Gene expression levels of MAO-A in SH-SY5Y and U-118 MG cells............................. 158

Fig. 49(b) Gene expression levels of MAO-B in SH-SY5Y and U-118 MG cells........................... 158

Fig. 50(a) Expression of MAO-A in U-118 MG cells following ethanol exposure.......................... 159

Fig. 50(b) Expression of MAO-B in U-118 MG cells following ethanol exposure.......................... 159

Fig. 51(a) MAO-A Expression in U-118 MG cells following exposure to tobacco compounds.... 160

Fig. 51(b) MAO-B Expression in U-118 MG cells following exposure to tobacco compounds.... 160

Fig. 52 Changes in MAO-A expression following treatment with mecamylamine and ethanol.

Fig. 53 Changes in MAO-A gene expression following treatment with tobacco compounds in conjunction with mecamylamine.

Fig. 54(a) elF4A2 gene expression in cells treated with ethanol and mecamylamine............

Fig. 55(a) Western blot of MAO-A staining from SH-SY5Y cells......

Fig. 55(b) Western blot of MAO-B staining from SH-SY5Y cells..

Fig. 55(c) Relative band densities on MAO-A Western blots...

Fig. 55(d) Relative band densities on MAO-B Western blots

\section{Chapter Seven}

Fig. 56 Schematic diagram of dopamine and opioid interactions

Fig. 57(a) Representative RT-PCR melt-curve for MOR and POLR2F amplicons........................... 183

Fig. 57(b) MOR PCR products identified by agarose gel electrophoresis...................................... 183

Fig. 58 Changes in MOR gene expression in SH-SY5Y cells following ethanol treatment.......

Fig. 59 Changes in MOR gene expression in SH-SY5Y cells treated with tobacco extract for up to 5 days.

Fig. 60(a) Effect of nicotine exposure on MOR gene expression in SH-SY5Y cells.

Fig. 60(b) Effect of HTPM exposure on MOR gene expression in SH-SY5Y cells.......................... 186

Fig. 60(c) Effect of QTPM exposure on MOR gene expression in SH-SY5Y cells......................... 186

Fig. 60(d) Summary of changes in MOR gene expression in SH-SY5Y cells treated with tobacco extracts........................................................................................................ 186

Fig. 61 MOR expression in cells treated for 5 days continuously or by refreshed regimen...

Fig. 62(a) Changes in MOR gene expression following treatment with mecamylamine..............

Fig. 62(b) MOR gene expression following treatment with mecamylamine and tobacco extracts.

\section{Chapter Nine}

Fig. 64(a) PCR Efficiency plot for MAO-A primers.......

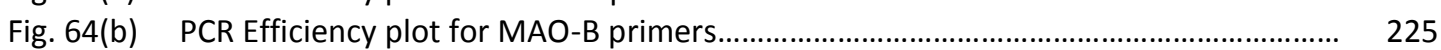

Fig. 64(c) PCR Efficiency plot for POLR2F primers................................................................... 226

Fig. 64(d) PCR Efficiency plot for M-RIP primers......................................................................... 226

Fig. 64(e) PCR Efficiency plot for GAPDH primers..................................................................... 226

Fig. 64(f) PCR Efficiency plot for elF4A2 primers.................................................................. 226

Fig. 64(g) PCR Efficiency plot for MOR primers......................................................................... 226 
- xiv - 


\section{Index of Tables}

Table $1 \quad$ Therapeutic monoamine oxidase inhibitors.............................................................. 15

Table 2 Monoamine oxidase inhibitors identified in tobacco smoke....................................... 31

Table $3 \quad$ Primary antibodies used for Western blotting............................................................. 53

Table 4 Secondary antibodies used for Western blotting......................................................... 53

Table $5 \quad$ Primer pairs used for qRT-PCR............................................................................... 58

Table $6 \quad I_{50}$ figures for clorgyline and pargyline inhibition............................................................ 78

Table $7 \quad$ Nicotine and tar data for cigarette brands tested.......................................................... 95

Table $8 \quad$ Nicotine and tar data for loose-leaf tobacco brands tested......................................... 96

Table 9 MAO-A IC 50 values of TPM samples and corresponding nicotine concentrations...... 98

Table 10 MAO-B IC 50 values of TPM samples and corresponding nicotine concentrations...... 99

Table 11 Tar concentrations of TPM samples.............................................................................. 100

Table 12 Enzyme reaction parameters for treated SH-SY5Y cells................................................. 121

Table 13 Efficiency of primer pairs used for qRT-PCR................................................................. 149

Table 14 MAO-A and MAO-B gene expression in ethanol treated SH-SY5Y cells....................... 152

Table 15 Summary of changes in MAO-A gene expression in SH-SY5Y cells.............................. 155

Table 16 Summary of changes in MAO-B gene expression in SH-SY5Y cells.............................. 157

Table 17 Comparison of MAO-A and MAO-B gene expression in U-118 MG and SH-SY5Y cells.

Table 18 MAO-A expression in SH-SY5Y cells treated with tobacco extracts and mecamylamine.

Table 19 Summary of MOR expression in ethanol treated SH-SY5Y cells................................... 184

Table 20 Summary of MOR gene expression in TPM treated SH-SY5Y cells............................... 187

Table 21 TPM sample numbering scheme and abbreviations................................................... 223 


\section{Abbreviations}

\begin{tabular}{|c|c|}
\hline BSA & Bovine serum albumin \\
\hline CNS & Central nervous system \\
\hline DA & Dopamine \\
\hline Da & Dalton \\
\hline DAT & Dopamine transporter \\
\hline $\mathrm{ddH}_{2} \mathrm{O}$ & Double-distilled water \\
\hline DEPC & Diethyl-pyro carbonate \\
\hline DOR & Delta Opioid Receptor \\
\hline elF4A2 & Eukaryotic initiation factor $4 \mathrm{~A}$ isoform 2 \\
\hline EtOH & Ethanol \\
\hline FCS & Fetal Calf Serum \\
\hline GABA & Gamma-aminobutyric acid \\
\hline GAPDH & Glyceraldehyde-3-phosphate dehydrogenase \\
\hline GC-MS/MS & Gas chromatography with tandem mass spectrometry \\
\hline HPLC & High performance liquid chromatography \\
\hline HTPM & Total Particulate Matter extract from Holiday brand cigarettes \\
\hline kb & Kilobases \\
\hline MAO & Monoamine oxidase \\
\hline MAO-A & Monoamine oxidase $\mathrm{A}$ \\
\hline MAO-B & Monoamine oxidase B \\
\hline MOR & Mu Opioid Receptor \\
\hline M-RIP & Myosin phosphatase-Rho interacting protein \\
\hline NAcc & Nucleus Accumbens \\
\hline nAChR & Nicotinic Acetylcholine Receptor \\
\hline NET & Norepinephrine transporter \\
\hline Nic & Nicotine \\
\hline Oprm & Mu Opioid Receptor gene \\
\hline PBS & Phosphate Buffered Saline (refer Appendix I) \\
\hline PCR & Polymerase Chain Reaction \\
\hline POLR2F & Polymerase (RNA) II (DNA directed) polypeptide F \\
\hline qRT-PCR & Quantitative real-time polymerase chain reaction \\
\hline QTPM & Total Particulate Matter extract from Quest ${ }^{\circledR}$ denicotinized cigarettes \\
\hline RPMI-1640 & Roswell Park Memorial Institute medium solution-1640 \\
\hline RT & Reverse Transcription \\
\hline RT & Room temperature \\
\hline RT-PCR & Reverse transcription - polymerase chain reaction \\
\hline SDS & Sodium Diodecyl Sulphate \\
\hline TBS & Tris Buffered Saline (refer Appendix I) \\
\hline TPM & Total Particulate Matter \\
\hline VTA & Ventral Tegmental Area \\
\hline
\end{tabular}




\section{Chapter One: Literature Review}

Smoking is the leading cause of preventable illness in the world today. It is well known that cigarette smoking increases the risks of developing numerous disorders, including cardiovascular disease, stroke, hypertension, cancer, and respiratory disease. These and other smoking-related illnesses are responsible for approximately five million deaths globally each year (N. L. Benowitz, 2008).

Effective smoking cessation aids are essential to assist in reducing the prevalence of cigarette smoking and smoking-related illness. Nicotine replacement therapies (NRT) are the most widely used form of pharmacological intervention, but have proven to be remarkably unsuccessful (Balfour \& Fagerstrom, 1996). Some studies report only 7\% of participants successfully quit smoking after 6 months treatment with NRT (Jarvik et al., 2000). This is indicative of the complex nature of tobacco dependence and suggests that factors other than addiction to nicotine are responsible for the establishment and maintenance of tobacco addiction.

Anecdotal evidence suggests that tobacco smoking is one of the hardest addictions to break. Some studies have reported that of those individuals who have smoked at least one cigarette in their lifetime, almost one third will develop a dependence on tobacco (Okuyemi et al., 2000). Although the majority of smokers have expressed a desire to give up smoking - surveys show that up to $70 \%$ of smokers have made attempts to quit - few are successful in their attempts. Nicotine is accepted as the principal neurobiologically active compound in a cocktail of over 4000 chemical compounds found in tobacco smoke (Fowles \& Dybing, 2003). However; a number of the non-nicotinic compounds in tobacco smoke have been identified, and show psychopharmacologically relevant activity in the brain.

Although little information is known about how these compounds may interact to influence tobacco addition, it is well known that the activity of monoamine oxidase (MAO), a key player in the neurochemistry of many behaviors, is significantly inhibited in tobacco smokers (J. S. Fowler et al., 1996a; J. S. Fowler et al., 1996b). Cigarette smokers show lowered levels of MAO activity in the brain and peripheral organs compared to ex-smokers and non-smokers. 
Since in vitro studies have shown that nicotine alone does not inhibit MAO activity, other neurobiologically active compounds in tobacco smoke must inhibit MAO. Investigations into this phenomenon have identified several compounds within cigarette smoke that reversibly or irreversibly inhibit MAOs, and it has been proposed that this inhibition of MAO activity may contribute to the reinforcement of tobacco addiction. If this is indeed the case, MAO inhibitors show potential as effective pharmaceutical aids to assist with smoking cessation.

Additionally, recent evidence has suggested an indirect role for the $\mu$ opioid receptor (MOR) in reinforcing the addictive properties of nicotine and tobacco. MOR is directly involved in the establishment and maintenance of dependence to many drugs of abuse, including cocaine, and morphine, and may also contribute to tobacco dependence. This may occur through MOR's influence on the endogenous opioid system, which is known to be involved in addiction reinforcement and reward.

The mechanisms by which tobacco dependence is established and reinforced are not yet well understood, but may involve addictive compounds within cigarette smoke in addition to nicotine, or compounds that augment the addiction potential of nicotine directly or indirectly, such as through the inhibition of MAO. Further investigation into the effects of the nonnicotinic components of tobacco smoke on addictive behaviors and the contribution of these compounds to relapse following successful smoking cessation may in turn lead to the development of more successful smoking cessation therapies. 


\section{1 - BIOCHEMISTRY AND MODE OF ACTION OF NICOTINE}

Nicotine is a tertiary aminyl alkaloid that is inhaled from burning tobacco in small droplets mixed with tobacco particulate. These droplets are inhaled into the small airways and alveoli of the lung and are rapidly absorbed directly into the pulmonary blood stream, and from there, the arterial blood supply, quickly reaching the brain (N. L. Benowitz, 2008). Peak arterial plasma nicotine concentrations reach approximately $20-40 \mathrm{ng} / \mathrm{mL}(0.12-0.25 \mu \mathrm{M})$ in smokers very quickly after smoke inhalation, with the nicotine from a bolus of cigarette smoke quickly crossing the blood-brain barrier and reaching the brain in approximately 7 to 10 seconds (Balfour et al., 2000a).

When nicotine reaches the brain it binds to and stimulates nicotinic acetylcholine receptors (nAChRs). These are pentameric ligand-gated ion channels that normally bind acetylcholine and play a neuromodulatory role in the central nervous system (Balfour \& Fagerstrom, 1996). These receptors are expressed throughout the CNS, and are usually found on neurons presynaptically (Berrendero et al., 2002). They are known to regulate processes such as cell excitability and neurotransmitter release, and modulate physiological functions such as arousal, anxiety, pain sensation, appetite, and cognitive functioning (Gotti \& Clementi, 2004). Nicotintic AChR activation has been demonstrated to increase the release of dopamine (Pontieri et al., 1996), glutamate (McGehee et al., 1995), GABA, noradrenaline, and acetylcholine (Berrendero et al., 2002). Nicotinic AChRs are composed of 5 subunits, $2 \alpha$ and $3 \beta$-subunits, which are arranged to form a pore (Fig. 1a, 1b). Nine genes have been identified that code for nAChR subunits, designated $\alpha 2-\alpha 7$ and $\beta 2-\beta 4$, the products of which combine to form the nAChR ion channel (Laviolette \& van der Kooy, 2004; Wonnacott et al., 2005). A number of homo- and heteromeric receptor subtypes have been described, including the $\alpha 4 \beta 2$ receptor, the $\alpha 4 \beta 3$ receptor, and the $\alpha 7$ homomeric receptor subtype. The $\alpha 4 \beta 2$ receptor subtype shows a high affinity for nicotine and has a high relative abundance in the brain (Wonnacott et al., 2005). It is assumed, therefore, that this is the major receptor subtype involved in the action of nicotine in the central nervous system. Receptors containing the $\beta 2$ subunit are required for dopamine release, which is critical for the reinforcing effects of nicotine, while the $\alpha 4$ subunit modulates nicotine sensitivity (N. L. Benowitz, 2008).

Post-mortem studies of human brain have found that smokers show widespread up-regulation of nAChRs, particularly the $\alpha 4 \beta 2$ receptor subtype, compared to non-smokers. This is thought to be a consequence of receptor desensitization due to chronic exposure to nicotine (Wonnacott et al., 2005). The nAChR exists in three possible conformations: the resting state, 
the active state and the desensitized state. The resting state receptor shows a low agonist affinity and requires relatively high concentrations of nicotine to cause opening of the ion channel. However, chronic stimulation of nAChRs with low concentrations of nicotine or other agonists results in receptor desensitization, a state in which the receptor will no longer respond to agonist binding. Desensitized receptors show a much higher affinity for nicotine than resting receptors, and as a result, nicotine binding stabilizes the receptor in the desensitized state (Quick \& Lester, 2002). It has been proposed that receptor desensitization on noradrenergic neurons may reduce the amount of noradrenaline released, and play a role in the 'tranquilizing' effects of cigarette smoke reported by many smokers. Desensitization is accompanied by an increase in receptor density due to a decreased rate of internalization and degradation of the receptor. Studies in animals have confirmed that chronic nicotine exposure causes desensitization of nAChRs (Wonnacott et al., 2005). It is thought that nAChR desensitization and up-regulation may play a role in synaptic plasticity and learning, and likely contributes to nicotine-mediated reward systems (Mansvelder et al., 2002; Quick \& Lester, 2002).

(a)

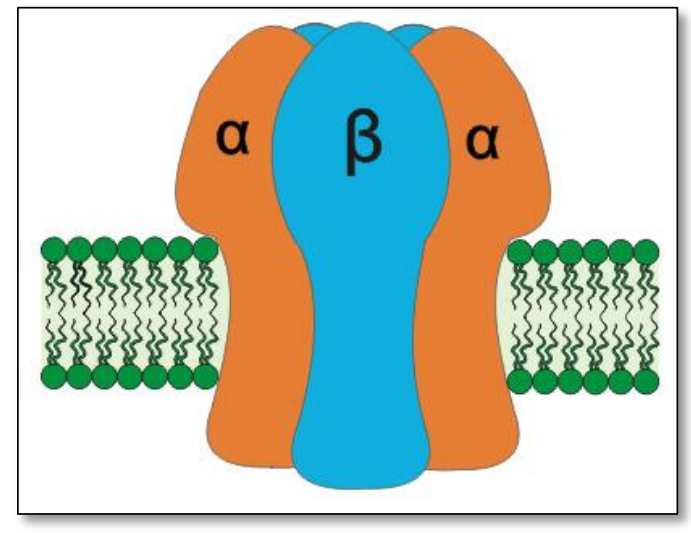

(b)

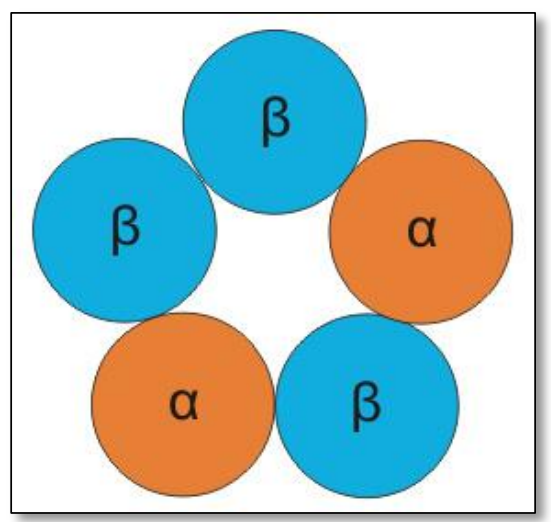

Figure 1: (a) Diagrammatic representation of the nicotinic acetylcholine receptor subtype localization in the plasma membrane. (b) $\alpha$ and $\beta$ subunits of nicotinic acetylcholine receptors are arranged in pentameric fashion to form a pore.

Nicotinic AChRs are found on the pre-synaptic cell membrane of many neurons, including glutamatergic, dopaminergic, and noradrenergic neurons in the mesolimbic areas of the brain (Laviolette \& van der Kooy, 2004). Nicotine binds to pre-synaptic nAChRs through the $\alpha$ subunit of the receptor, which leads to a conformational change that opens the cation channel. This causes the influx of $\mathrm{Na}^{+}, \mathrm{K}^{+}$, and $\mathrm{Ca}^{2+}$ ions (Wonnacott et al., 2005), which in turn leads to activation of voltage-dependent calcium channels, and further influx of $\mathrm{Ca}^{2+}$ ions 
(N. L. Benowitz, 2008). Activation of neuronal nAChRs leads to the release of a host of neurotransmitters, including dopamine, norepinephrine, serotonin, GABA, acetylcholine, and $\beta$-endorphin. The various psychological and physiological effects of nicotine are mediated by the downstream actions of these neurotransmitters. Physiologically, nicotine induces acute increases in heart rate and blood pressure, vasoconstriction in the skin and coronary arteries, and vasodilation in other areas, including skeletal muscle (Balfour et al., 2000a). However, chronic nicotine use builds considerable tolerance to these physiological effects. Psychologically, nicotine is known to elicit a pleasurable response, caused by increased dopamine release. Additionally, nicotine increases arousal and cognitive enhancement, through increases in norepinephrine and acetylcholine concentrations, and elevates mood, and suppresses appetite due to increased serotonin release. It also reduces anxiety and pain through GABA ( $\gamma$-aminobutyric acid) and $\beta$-endorphin release (N. L. Benowitz, 2008).

Although nicotine causes complex changes in the concentrations of a number of neurotransmitters in the brain, the dopaminergic system has been studied extensively because of its proposed role in addictive behaviors. Nicotine is known to increase the concentration of dopamine in the brain. Positron emission tomography (PET) scanning has confirmed that dopamine activity is increased in the basal ganglia of smokers compared to non-smokers (Salokangas et al., 2000), and that cigarette smoking elevates dopamine concentrations in these mesolimbic brain areas (Barrett et al., 2004; Brody et al., 2004). This has also been confirmed by microdialysis studies in animals, which have found that physiologically relevant concentrations of nicotine increase extracellular dopamine concentrations in the nucleus accumbens by two-fold (Di Chiara \& Imperato, 1988; Pontieri et al., 1996).

The mesolimbic areas of the brain include dopaminergic neurons projecting from the ventral tegmental area (VTA) in the brainstem to the nucleus accumbens and caudate putamen (in the striatum), prefrontal cortex, and amydala (Betz et al., 2000; Contet et al., 2004). Both high and low affinity nAChRs have been identified on the cell bodies and terminals of dopaminergic neurons in these regions (Balfour, 2002; Pontieri et al., 1996), as well as on inhibitory GABAergic neurons and excitatory glutamatergic afferents that project to the VTA (Mansvelder et al., 2002; Pidoplichko et al., 2004). Nicotine stimulates pre-synaptic nAChRs on dopaminergic neurons in the ventral tegmental area (VTA) (Pidoplichko et al., 2004) - an area of the brain known to be involved in addiction and reward mechanisms. These neurons project to the shell of the nucleus accumbens, and their activation releases dopamine in that region 
(Corrigall et al., 2000). The ability to cause dopamine overflow in the nucleus accumbens has been linked to many drugs of abuse, including nicotine, amphetamine, and cocaine (Pontieri et al., 1996), and is critical to the reinforcing properties of these drugs. It has been proposed that this mechanism confers the addictive potential of the drugs, and these mechanisms will be discussed in greater detail in section 1.2 of this thesis.

Nicotine activation of dopamine neurons in the VTA is enhanced by glutamatergic afferents, and inhibited by GABA-ergic projections to this area (N. L. Benowitz, 2008). These glutamatergic neurons express nAChRs that bind nicotine, and do not readily desensitize at physiologically relevant concentrations, thus enhancing dopaminergic excitation. GABA-ergic neurons projecting to the VTA inhibit dopamine excitation in this area; however, nicotine also binds to nAChRs on these GABA-ergic neurons. These receptors are quickly desensitized by nicotine, decreasing the GABA inhibition of dopamine neurons. These two mechanisms work together to prolong the firing of dopaminergic neurons in response to nicotine (Pidoplichko et al., 2004), potentially increasing nicotine's addictive potential.

Chronic exposure to nicotine also causes a reduction in serotonin concentration in the hippocampus (Balfour \& Ridley, 2000). This reduction is limited strictly to the hippocampus and is associated with reduced rates of serotonin synthesis. The consequences of this localized serotonin reduction have yet to be elucidated; however, it has been proposed that reduced serotonin may be associated with the reduced anxiety felt after smoking a cigarette, as reported by smokers. Chronic reduction of serotonin release in the hippocampus causes up-regulation of $5-\mathrm{HT} 1_{\mathrm{A}}$ receptors in this region, and stimulation of these receptors has been implicated in the onset of anxiety. It has been proposed that upon cessation of smoking, serotonin release is no longer suppressed by cigarette smoke. Thus, increased serotonin levels stimulate $5 \mathrm{HT} 1_{\mathrm{A}}$ receptors that are present at high density in the hippocampus (Balfour \& Ridley, 2000), causing the smoker to experience increased anxiety due to nicotine withdrawal.

While nicotine binds to nAChRs, stimulating dopaminergic, serotonergic and noradrenergic neurons to fire, and increases the concentrations of monoaminergic neurotransmitters in areas of the brain associated with mood, pleasure, and reward, inhibition of the enzyme MAO by compounds within cigarette smoke leads to further increases in the concentrations of these neurotransmitters. Upon stimulation of $\mathrm{nAChRs}$ by nicotine, catecholamine neurotransmitters such as dopamine and norepinephrine are released from the terminal bouton of the neuron into the synaptic cleft (Salminen et al., 2004; Wonnacott et al., 2005). 
The neurotransmitters bind to post-synaptic receptors, stimulating the neuron to fire (Elhwuegi, 2004). The action of catecholamines in the synaptic cleft are terminated by selective re-uptake of the neurotransmitter into the pre-synaptic terminal via $\mathrm{Na}^{+}$dependent transporters such as the dopamine transporter (DAT) and the norepinephrine transporter (NET). Once inside the terminal these catecholamines are largely re-loaded into synaptic vesicles, but may also be degraded by MAO (Figure 2). Astrocytes envelop the synaptic cleft, providing support to the neurons and preventing leakage of neurotransmitter into surrounding areas. Dopamine is also transported into astrocytes and glial cells through the actions of NET and the extraneuronal monoamine transporter (EMT) (Takeda et al., 2002). Since astrocytes also demonstrate high levels of MAO activity, it has been proposed that they assist in terminating the actions of neurotransmitters at the synaptic cleft by selective uptake and intracellular degradation.

Thus, the nicotine in cigarette smoke enhances the release of catecholaminergic neurotransmitters, and in particular, augments dopamine release and availability in the nucleus accumbens, a brain area which has been implicated in pathways of reward, reinforcement, and addiction. MAO inhibitors in tobacco smoke may act in synergy with nicotine to maintain elevated concentrations of catecholamines and dopamine at their sites of action by reducing the rate of degradation by MAO. 


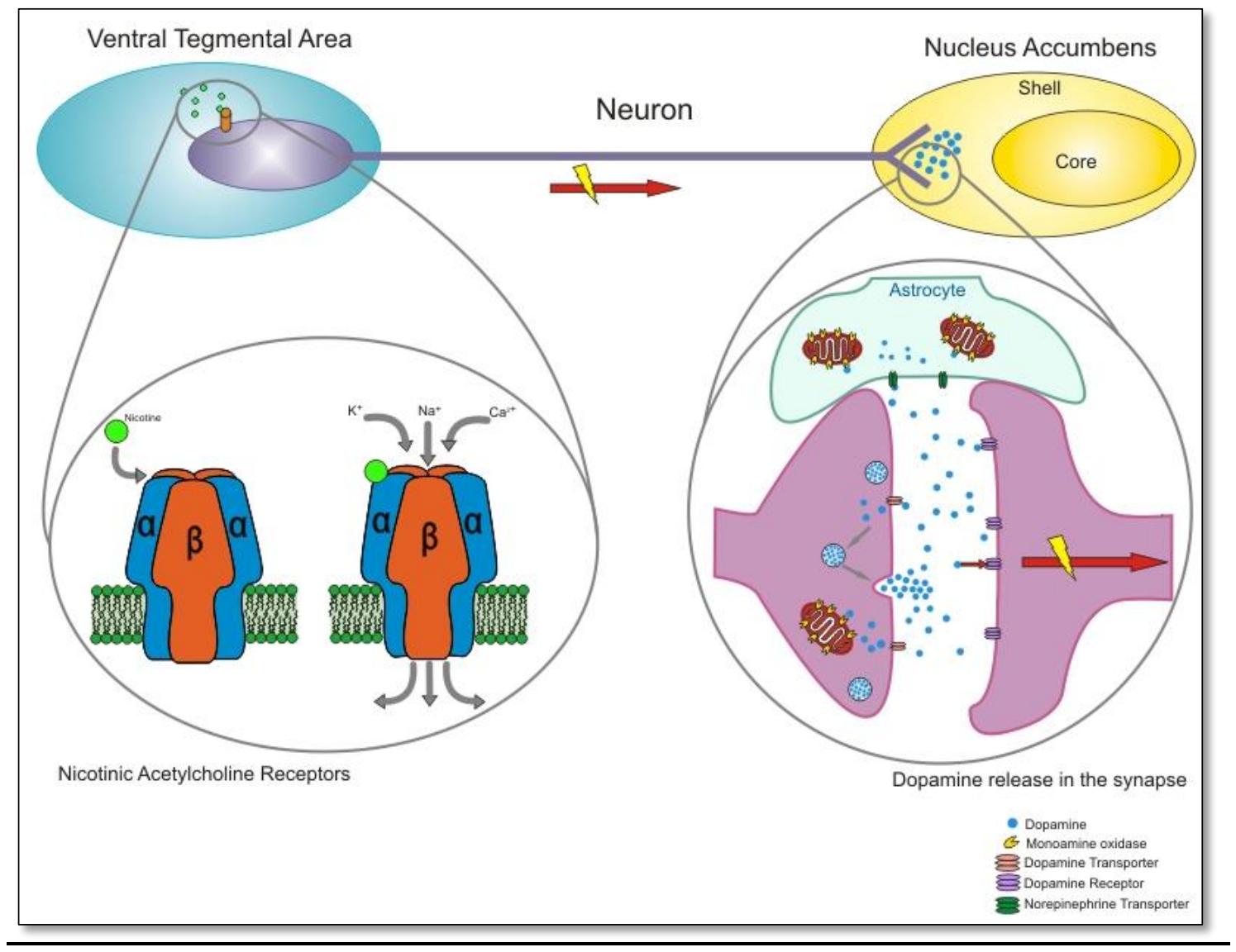

Figure 2: Nicotine binds to nAChRs, causing a conformational change and opening of the cation channel. Sodium, potassium and calcium cations flow into the neuron, causing depolarization and neuronal firing. Dopamine released at the synapse transmits the signal post-synaptically before reuptake by DAT. MAO acts to degrade dopamine in the neuron or astrocytes which surround the synapse. 


\section{2 - MECHANISMS OF ADDICTION}

Nicotine displays a number of psychopharmacological characteristics in common with other addictive drugs, such as cocaine and amphetamines. Nicotine administration causes arousal and increased locomotor activity and elicits pleasant or rewarding effects in the brain that reinforce self-administration behavior. Following chronic exposure to nicotine, cessation of administration causes a withdrawal syndrome that an addict seeks to avoid by continued administration of the drug (Balfour, 2002; N. L. Benowitz, 2008). Interestingly, nicotine alone is a relatively weak reinforcer in animal models of addiction compared to other drugs of abuse such as amphetamine and cocaine (Benwell \& Balfour, 1992). The neurobiological mechanisms underpinning drug addiction and the psychoactive properties of nicotine are very complex and knowledge of these mechanisms is still developing.

Drug addiction is best described as a chronic relapsing disorder of compulsive drug use without a medical reason that results from gradual adaptations of the brain to repeated drug exposure (Betz et al., 2000; Contet et al., 2004). Current understanding suggests that drug addiction arises from repeated and abnormal stimulation of the brain's natural reward pathways by exogenous compounds, leading to deregulation of these reward pathways. This manifests as drug dependence, tolerance, drug-seeking behaviour, drug-craving, and withdrawal. The functioning of reward pathways in both natural and addiction paradigms involves many neurotransmitter systems, including the dopaminergic system and the endogenous opioid system - both of which have been studied extensively for their roles in establishing and maintaining addiction.

Naturally reinforcing stimuli such as food, water, and sex have the ability to motivate complex behaviours and increase dopamine transmission in the nucleus accumbens (Tanda \& Di Chiara, 1998), an area of the brain that is intrinsically linked with mechanisms of reward and reinforcement. Exogenous drugs abused by humans, including nicotine also induce dopamine release in the nucleus accumbens (Balfour et al., 2000b; Pidoplichko et al., 1997; Pontieri et al., 1996). It is widely held that drugs of abuse activate the same reward pathways and neurotransmitter systems as natural reinforcers, but while natural reinforcers activate these pathways through indirect peripheral sensory mechanisms, drugs of abuse activate these pathways in the brain by direct neurochemical means (Tanda \& Di Chiara, 1998). The direct neurochemical activation of these pathways could explain why drugs like morphine, cocaine, and nicotine are so addictive but food generally is not. These neurobiological mechanisms of 
addiction, reward, and reinforcement have been studied by many methods, including direct monitoring of the neurotransmitter systems involved, usually through microdialysis and electrophysiology studies. Other types of investigation involve pharmacologic manipulation of receptor systems, by careful application and comparison of the effects of agonists and antagonists, as well as behavioural studies in animal models of addiction.

Although drugs abused by humans have diverse pharmacological properties (for example CNS depressants, psychostimulants, and analgesics), and act primarily through varied mechanisms, there is strong evidence that they all activate a common dopaminergic pathway that mediates the rewarding properties of the drug, and establishes drug reinforcement and dependence. Drugs of abuse elicit increased dopamine transmission in the nucleus accumbens, activating the mesolimbic dopamine pathway. The mesolimbic areas of the brain include the nucleus accumbens, caudate putamen, ventral tegmental area, substantia nigra, pre-frontal cortex, and the basolateral amygdala (McGehee, 2006). Specifically, the dopamine pathway links the ventral tegmental area in the midbrain with the nucleus accumbens in the striatum. These brain regions are thought to mediate pleasure, motivation, and reward and facilitate behavioural responses to rewarding stimuli (Balfour, 2002).

Dopamine plays a neuromodulatory role in the central nervous system and exerts its effects through binding to the G-protein-coupled dopamine receptors D1 and D2. This important neurotransmitter is thought to play different roles in mediating behaviour depending on the brain area where it is released. The nucleus accumbens is an area of the midbrain that has repeatedly been shown to be involved in mechanisms of reward and addiction. Natural stimuli are known to increase dopamine transmission in this region, but drugs of abuse have been shown to elicit larger and more prolonged increases in dopamine release. It is thought that this promotes the acquisition of behaviours that result in drug administration and establishment of dependence (Balfour, 2002).

Electrophysiological studies have shown that resting midbrain dopamine neurons fire at low frequency $(\sim 4 \mathrm{~Hz}$ ), maintaining dopaminergic tone (Britt \& McGehee, 2008), but change to a burst firing pattern in response to rewarding stimuli. This change to a rapid pattern of neuronal firing overwhelms dopamine transporters (DAT) in the area, leading to increased extracellular dopamine concentrations and dopamine overflow. In the striatum, nicotine has been demonstrated to inhibit dopamine overflow induced by low-frequency neuronal firing but elicits dopamine overflow by stimulating burst firing (Britt \& McGehee, 2008). Behavioural studies have shown a relationship between rapid burst firing in dopamine 
neurons and discrete learning events. It is thought that burst firing and dopamine overflow in the nucleus accumbens may contribute to the acquisition and establishment of drug dependence.

The nucleus accumbens (NAcc) can be divided into two areas, the shell and the core, which are anatomically and functionally distinct (Balfour, 2002). The shell is an extension of the amygdala, which is associated with pathways of motivation and reward; whereas, the core is related to the striatum and has neuronal projections to areas of the brain involved in motor function control (Balfour et al., 2000b). It has been reported that drugs of abuse preferentially increase dopamine transmission in the outer shell of the NAcc (Di Chiara et al., 2004; Pontieri et al., 1996), and this is associated with the reinforcing potential of the drug. In contrast, dopamine release in the core of the accumbens has been associated with the behavioural sensitization of the locomotor effects induced by drugs of abuse. In common with other psychostimulant drugs, nicotine causes increased locomotor activity in laboratory animals at doses relevant to human use (Benwell \& Balfour, 1992; Cadoni \& Di Chiara, 2000). Repeated exposures to nicotine progressively and significantly increase locomotor activity, referred to as behavioral sensitization. Benwell \& Balfour (1992) observed a dose-dependent increase in locomotor activity in rats treated acutely with nicotine, increasing activity by 100 $150 \%$. Following 5 days pre-treatment with nicotine, locomotor activity increased $300 \%$ in rats given a challenge dose of nicotine, and extracellular dopamine concentrations in the accumbens were measured at $200 \%$ of baseline by microdialysis. Cadoni \& Di Chiara (2000) later observed increased locomotor activity in nicotine pre-treated rats compared to saline pre-treated animals, when given a challenge dose of nicotine. This was correlated with significantly increased dopamine release in the accumbal core, when compared to salinepretreated rats. However, nicotine sensitized animals showed lower levels of dopamine release in the shell of the accumbens compared to their saline treated counterparts (Cadoni \& Di Chiara, 2000).

While dopamine release in the shell of the accumbens has been correlated with selfadministration and reinforcement of drugs of abuse, it is still unclear what role this mechanism might play in establishing addiction. It has previously been widely assumed that this dopamine release mediates the pleasurable or euphoriant properties of psychostimulant drugs; however, this view is changing (Balfour et al., 2000b). It has been suggested that the primary role of dopamine increase in the accumbal shell is to facilitate stimulus-reward associations and the attribution of positive incentive salience to neutral cues associated with 
drug reward. That is, increased drug administration results in greater motivation and desire to receive more drug reward. Balfour (2000b) and Di Chiara (1998) propose that addiction to psychostimulant drugs is linked to overflow of dopamine in the nucleus accumbens that is greater and more prolonged than that elicited by natural rewards, and as a result, cues and activities associated with repeated drug-taking achieve abnormally strong salience. Additionally, in a study conducted by Tanda and Di Chiara (1998) into the stimulation of dopamine transmission in the rat nucleus accumbens it was postulated that dopamine release in the nucleus accumbens was associated with relative novelty of the stimulus. This study found dopamine release in the accumbens was elicited by nicotine administration, but also by a novel palatable food source. It was noted that the dopamine response elicited by food showed rapid habituation; whereas, the dopamine response to nicotine did not. Therefore, Tanda \& Di Chiara (1998) hypothesized that accumbal dopamine release was associated with acquisition of reward motivation and positive motivational learning. This leads to otherwise neutral stimuli becoming incentives for motivated behaviours as a result of their association with the rewarding stimuli. That is, dopamine release in the accumbens may facilitate the acquisition and maintenance of drug-seeking behaviours, in agreement with the previous hypothesis stated by Balfour (2000b) and Di Chiara (1998). Tanda \& Di Chiara (1998) suggest that food is not generally an addictive stimulus because of the rapid habituation to this stimulus, but in contrast, drugs of abuse activate dopamine transmission, causing greater and more sustained overflow with repeated administration. This is thought to result in strengthening of abnormal motivational learning processes resulting in drug-seeking behavior.

In addition to the dopamine hypothesis of dependence, it has been proposed that inhibition of MAO by compounds in cigarette smoke contributes synergistically with nicotine to reinforce addiction by maintaining increased dopamine concentrations in brain regions associated with addiction and reinforcement. Recent in vivo research in rats has supported this hypothesis (A. S. Villegier et al., 2003). In this study, behavioral sensitization was reported in rats treated with D-amphetamine, nicotine, or nicotine in conjunction with the irreversible MAO-B inhibitors pargyline or tranylcypromine. As discussed above, increased dopamine release in the accumbens induced by $\mathrm{D}$-amphetamine, nicotine, and other drugs of abuse causes locomotor hyperactivity in rats, and repeated exposure causes behavioural sensitization. Villegier et al. showed that a challenge dose of D-amphetamine induced behavioral sensitization in pre-treated rats after up to 30 days withdrawal. However, 30 days of withdrawal abolished behavioral sensitization in nicotine-treated rats. It was noted that nicotine alone induced only a transient behavioral sensitization, but when nicotine was co- 
administered with the MAO inhibitors tranylcypromine or pargyline, behavioral sensitization to nicotine was maintained for up to 90 days. This suggests that MAO inhibitors may contribute to the addictive potential of nicotine in tobacco dependence.

More recently, it was reported that inhibition of MAO significantly increased the motivation of rats to self-administer nicotine (Guillem et al., 2005). This study utilized an animal selfadministration model in which rats are trained to perform a task (usually pressing a lever or poking their nose through a hole) to receive a dose of drug through an indwelling catheter. This model can be used to assess the relative reinforcing properties of drugs of abuse by recording the number of lever presses the animal will perform in order to receive a drug reward (Roth-Deri et al., 2008). Rats that were pre-treated with tranylcypromine and phenelzine freely self-administered more nicotine than placebo-treated animals. It was also noted that rats treated with MAO inhibitors were willing to work harder by performing more lever presses to obtain a dose of nicotine than control rats. This study confirmed that MAO inhibitors did not alter the pharmacodynamic properties of nicotine and did not interfere with the psychostimulant action of nicotine, but they dramatically increased nicotine's reinforcing properties (Guillem et al., 2005). These results suggest that the MAO inhibitory action of nonnicotinic compounds in tobacco smoke may act synergistically with nicotine to establish and maintain tobacco dependence by increasing nicotine's reinforcement potential.

\section{3 - INTRODUCTION TO MONOAMINE OXIDASE}

\subsection{1-General Introduction}

Since the discovery that inhibitors of MAO have an anti-depressive effect (Edmondson et al., 2004b; Pare \& Sandler, 1959; Sainz, 1960), extensive research has been conducted into the function and influence of these enzymes in the mammalian central nervous system. Monoamine oxidases are flavin adenosine dinucleotide (FAD)-containing enzymes that are integral proteins found in the mitochondrial outer membrane (Abell \& Kwan, 2001; Binda et al., 2002). MAO catalyses the oxidative deamination of many biogenic amines and plays a vital role in the regulation of neurotransmitters in the mammalian central nervous system, including regulation of synaptic concentrations of serotonin, dopamine, norepinephrine and other catecholaminergic neurotransmitters. The concentrations of these neurotransmitters 
contribute to the regulation of mood, movement, memory and arousal (Nagatsu, 2004). MAO also has an important function in breaking down exogenous sympathomimetic amines ingested in the diet, including tyramine, tryptamine and phenylethylamine (Abell \& Kwan, 2001; Berry et al., 1994; Weyler et al., 1990).

In the reaction catalyzed by MAO the monoamine substrate binds to the active site on the enzyme and is oxidised to an imine intermediate, with the covalently bound FAD being reduced to $\mathrm{FADH}_{2}$. $\mathrm{FADH}$ 2 then reacts with molecular oxygen and converted to FAD, producing hydrogen peroxide as a by-product (Fig. 3). The imine dissociates from MAO and is hydrolysed to form an aldehyde intermediate and $\mathrm{NH}_{4}{ }^{+}$(Edmondson et al., 2009). The aldehyde is then rapidly oxidized by aldehyde dehydrogenase or aldehyde reductase.

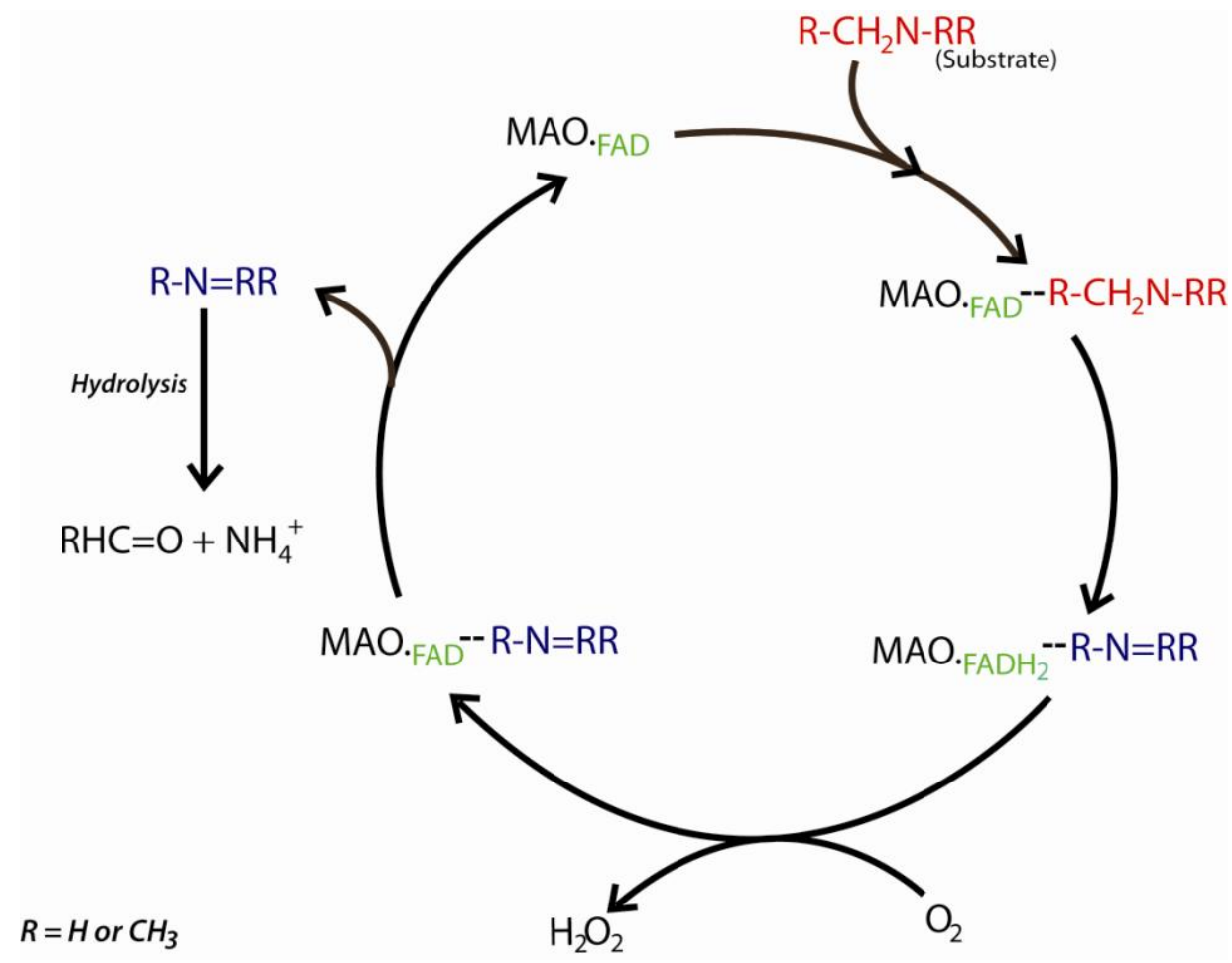

Figure 3: The reaction catalyzed by monoamine oxidase. A monoamine substrate is oxidatively deaminated to form an aldehyde product with hydrogen peroxide as a by-product.

Two isoforms of MAO are known to exist in mammals, designated MAO-A and MAO-B. These isoforms were originally distinguished by their differences in substrate and inhibitor specificities. MAO-A preferentially deaminates serotonin and norepinephrine; whereas, MAOB catabolises phenylethylamine and benzylamine. Dopamine, tyramine and tryptamine are 
metabolized by both isozymes, depending on enzyme and substrate concentrations (O'Carroll et al., 1983). These substrate specificities are not absolute but are relative to the concentration of substrate; thus, MAO-A and B substrate and inhibitor specificities overlap at high substrate concentrations under favourable conditions (Berry et al., 1994; Konradi et al., 1989). No compound has yet been identified that shows absolute specificity for either isozyme. However, MAO-A is selectively and irreversibly inhibited by clorgyline and moclobemide at low concentrations; whereas, MAO-B is specifically inactivated by deprenyl and pargyline at low concentrations (Yamada \& Yasuhara, 2004) (Table 1). Despite the limitation of non-specificity at high concentrations, these inhibitors have been used successfully to distinguish the activity of each enzyme in vivo and in vitro.

Dysfunction of MAO is thought to be associated with the etiology of a number of neurological disorders, including Parkinson's disease (Baron, 1986; Checkoway et al., 1998; Morens et al., 1995), Alzheimer's disease (Emilsson et al., 2002; Riederer et al., 2004), Huntington's disease (Sandler et al., 1981), major depression (Covey et al., 1997; J. E. Rose et al., 2001), and schizophrenia (Simpson et al., 1999). MAO inhibitors are used therapeutically in humans for treatment of severe depression and treatment of the symptoms and progression of Parkinson's and Alzheimer's disease (Yamada \& Yasuhara, 2004).

Table 1: Therapeutic monoamine oxidase inhibitors

\begin{tabular}{|c|c|c|c|}
\hline Inhibitor & Inhibition & Clinical Application & Reference \\
\hline Phenelzine & $\begin{array}{l}\text { Non-selective, } \\
\text { irreversible }\end{array}$ & Antidepressant & (Guillem et al., 2005) \\
\hline Tranylcypromine & $\begin{array}{l}\text { Non-selective, } \\
\text { irreversible }\end{array}$ & Antidepressant & (Youdim \& Finberg, 1987) \\
\hline Clorgyline & $\begin{array}{l}\text { MAO-A, } \\
\text { irreversible }\end{array}$ & & (Yamada \& Yasuhara, 2004) \\
\hline Moclobemide & MAO-A, reversible & $\begin{array}{l}\text { Antidepressant; } \\
\text { Alzheimer's disease; Smoking } \\
\text { cessation? }\end{array}$ & $\begin{array}{l}\text { (Berlin et al., 1995a; } \\
\text { Yamada \& Yasuhara, 2004) }\end{array}$ \\
\hline $\begin{array}{l}\text { Deprenyl } \\
\text { (selegiline) }\end{array}$ & $\begin{array}{l}\text { MAO-B, } \\
\text { irreversible }\end{array}$ & $\begin{array}{l}\text { Parkinson's disease; } \\
\text { Alzheimer's disease; Smoking } \\
\text { cessation? }\end{array}$ & $\begin{array}{l}\text { (Biberman et al., 2003; Sano } \\
\text { et al., 1997; Yamada \& } \\
\text { Yasuhara, 2004; P. H. Yu et } \\
\text { al., 1992) }\end{array}$ \\
\hline Pargyline & $\begin{array}{l}\text { MAO-B, } \\
\text { irreversible }\end{array}$ & & (Yamada \& Yasuhara, 2004) \\
\hline
\end{tabular}




\subsection{2 - Enzyme Structure}

The primary protein structures of MAO-A and B have been deduced from sequence data following cDNA cloning. Both isozymes are highly conserved, with the amino acid sequences of the two isoforms showing 70\% sequence identity (Abell \& Kwan, 2001; Bach et al., 1988). Functional MAO-A and B proteins are thought to consist of two identical subunits each, with molecular weights of $59 \mathrm{kDa}$ and $58 \mathrm{kDa}$, respectively (Shih et al., 1999). FAD is covalently bound to the enzyme, and is essential for enzyme function. Each subunit is bound to one FAD molecule at a conserved pentapeptide sequence to form the active enzyme. The pentapeptide sequence comprises the residues Ser-Gly-Gly-Cys-Tyr, with the FAD molecule linked through the cysteine residue by a sulfhydryl bond. The FAD linkage site is located at position 406 in MAO-A and 397 in MAO-B (Bach et al., 1988; Z. Y. Chen et al., 1991; Edmondson et al., 2004a). This pentapeptide sequence is highly conserved in MAO proteins between species.

Studies using site-directed mutagenesis have identified four highly conserved regions believed to be vital for enzyme function (Shih et al., 1999). These comprise the FAD attachment site described above, a putative substrate binding domain, an ADP binding $\beta-\alpha-\beta$ site, and a Cterminal membrane-associated $\alpha$-helix (Bach et al., 1988; Shih et al., 1999). The crystal structure of MAO-B reveals that the C-terminal membrane-spanning $\alpha$-helix anchors the protein to the mitochondrial outer membrane (Binda et al., 2004).

The substrate binding domain is thought to be located at residues 178-221, and replacement of residues 161-375 of MAO-A with those of MAO-B changes the substrate and inhibitor specificity of the enzyme to those of the B isoform (Shih et al., 1999). Using rat MAO it was found that substitution of Phe-208 in MAO-A with Ile, as found at this position in MAO-B, was also sufficient to change the substrate selectivity of MAO-A to that of $B$, and the selectivity of MAO-B to A if lle was replaced with Phe at this position.

Although monoamine oxidases are integral membrane proteins found in the mitochondria, they are coded for by two distinct genes on the X chromosome (Grimsby et al., 1991). It has been confirmed that MAO is synthesized in the cytoplasm of the cell rather than in the mitochondrion. The synthesized protein contains a C-terminal non-cleavable targeting sequence that is inserted into the mitochondrial membrane by ubiquitin in an ATP-dependent process (Mitoma \& Ito, 1992; Zhuang et al., 1992). This 32-amino acid sequence forms the $\alpha$ helix that anchors the MAO protein to the mitochondrial membrane. Because of its vital 
importance in enzyme function, this sequence is highly conserved between $A$ and $B$ isoforms and between species (Binda et al., 2004; Edmondson et al., 2004b).

\subsection{3 - Gene Structure}

Human MAO-A and MAO-B are both located on the short arm of the $X$ chromosome at positions Xp11.23 and Xp22.1, respectively (K. Chen, 2004). The genes are arranged in tail-totail orientation and show identical intron-exon organization, comprising 15 exons each (Grimsby et al., 1991). This suggests that the MAO-A and B genes were derived by duplication of a single ancestral gene. Exon 12, which codes for the FAD-binding region, has been identified as the most highly conserved exon, showing $93.9 \%$ sequence identity between MAO-A and B.

The MAO-A and B gene promoter regions are also highly conserved, sharing approximately $60 \%$ sequence homology. Both promoters consist of GC-rich regions; however, organization of transcription elements between these two regions is notably different. The MAO-A promoter consists of three Sp1 elements, shows bi-directional promoter activity, and lacks a TATA box (Shih et al., 1999; Zhu et al., 1992). Three putative glucocorticoid response elements have also been identified, explaining why MAO-A expression has been found to increase after dexamethasone treatment. The MAO-B promoter contains two clusters of Sp1 elements separated by a CACCC motif (Zhu et al., 1992). It has been proposed that the different organization of MAO-A and B promoter regions may underlie the observed differences in tissue- and cell-specific expression (K. Chen, 2004; Zhu et al., 1992). 
(a)

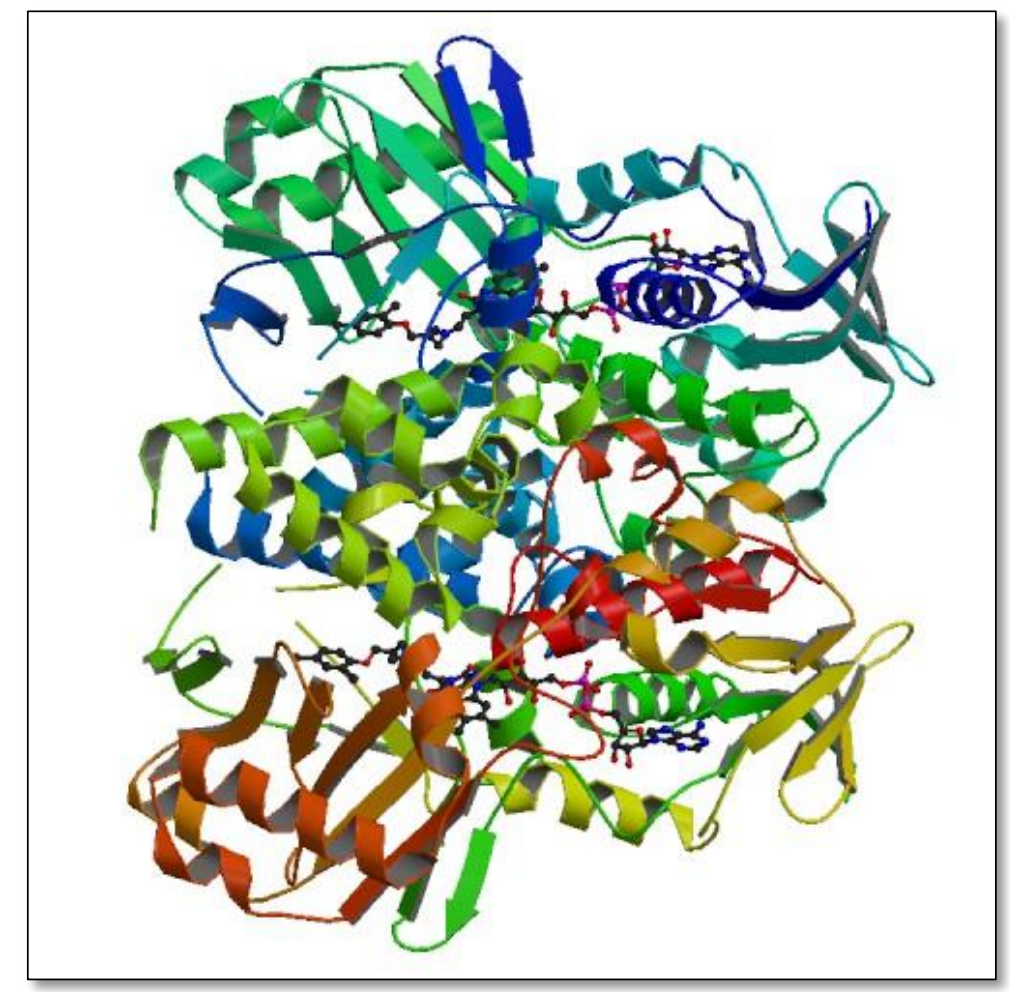

Figure 4(a): Molecular schematic of monoamine oxidase A. Two MAO$A$ units are positioned on top of each other, each with one molecule of FAD bound.

(Reproduced from De Colibus et al. (2005) Three dimensional structure of monoamine oxidase $A$ (MAOA): relations to the structures of rat MAOA and human MAOB. Proceedings of the National Academy of Sciences, USA, 102(36), 12684-12689.) (b)

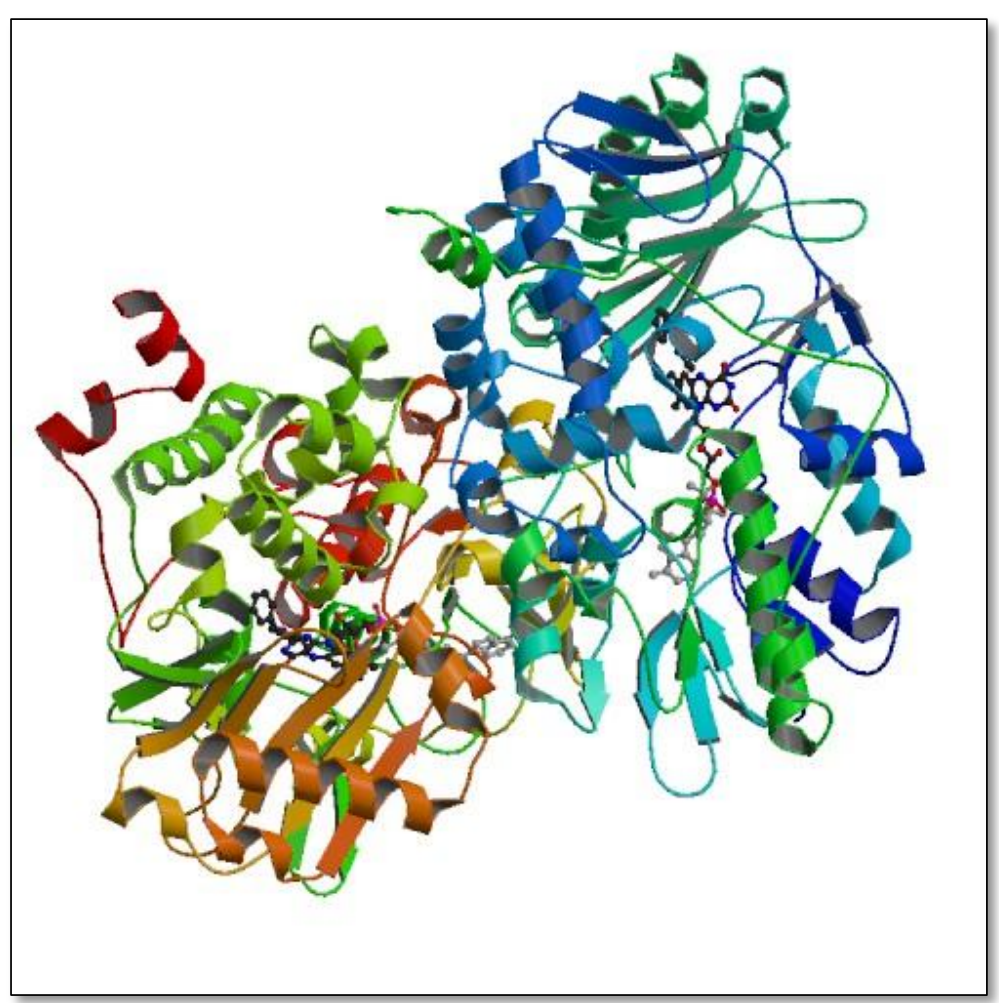

Figure 4(b): Molecular schematic of monoamine oxidase B. Two MAO $B$ molecules are positioned side by side here, each with one molecule of FAD bound.

(Reproduced from Binda et al. (2002) Structure of human monoamine oxidase $\mathrm{B}$, a drug target for the treatment of neurological disorders. Nature Structural Biology, 9(1), 22-26.) 


\subsection{4 - Localization and Activity of Monoamine Oxidase}

Monoamine oxidase activity has been found in the brain and peripheral tissues of all mammalian species that have been investigated to date (Weyler et al., 1990); however, expression of the two MAO isoforms shows species- and tissue-specific differences in their activity and localization. An understanding of the localization of MAO-A and B is important, since tissue and cellular compartmentalization of these enzymes determines to some degree the substrates to which they are exposed and therefore their action in vivo.

The activities and distributions of MAO isoforms in the central nervous system show regional and species-specific differences. Studies of MAO in rodents are characterized by differences in the distribution, abundance, and substrate binding affinity of each MAO isoform relative to those seen in humans (Berry et al., 1994; Weyler et al., 1990). Rodents have slightly higher levels of MAO-A than MAO-B protein in the brain; whereas, in human brain MAO-B is more abundant than MAO-A (Saura et al., 1992; Van Amsterdam et al., 2006). Because of these species-specific differences, caution should be exercised in extrapolating the results of studies in animal models to the human brain.

\subsubsection{1 - Localization in the Brain}

MAO-A and MAO-B show differential localization in the brain. This is thought to arise as a consequence of the differential distribution of the substrates for each enzyme. Although both MAO isoforms have been observed in almost all areas of the human brain, the relative abundance of each isoform shows region to region variability. Investigations into the distribution of MAO isoforms in human brain have been conducted using autoradiography (Richards et al., 1992; Saura et al., 1996a; Saura et al., 1992), immunohistochemistry (Thorpe et al., 1987; Westlund et al., 1988), enzyme histochemistry (Konradi et al., 1989), and in situ hybridization (Richards et al., 1992; Saura et al., 1996a), and there is generally good agreement in results between studies.

Several investigations using immunohistochemistry have identified the presence of MAO-A protein in catecholaminergic neurons in the human brain, in particular, adrenergic and noradrenergic neurons. Areas showing dense immunostaining for MAO-A include the locus coeruleus and subcoeruleus, nucleus accumbens, substantia nigra, ventral tegmentum, 
mammillary complex, and superior cervical ganglion (Westlund et al., 1988). Histochemical and in situ hybridization techniques have confirmed these distributions (Konradi et al., 1989; Saura et al., 1996a). Low levels of staining are generally observed in all white matter areas, with the lowest levels of MAO-A expression being reported in the cerebellum and frontal cortex.

MAO-B specific histochemical and hybridization staining is localized to serotonergic and histaminergic neurons (Konradi et al., 1989; Saura et al., 1996a). MAO-B activity and expression is highest in the dorsal and medial raphe complex, substantia nigra, and hypothalamus, and moderate levels have been detected in the reticular formation, nucleus centralis, caudate putamen, and granule cells of the dentate gyrus. As observed with MAO-A, the lowest levels of activity of MAO-B are found in the white matter areas of the brain, as well as the hippocampus and occipital cortex.

While MAO-B is known to be localized to neuronal cells in the substantia nigra, raphe complex and hypothalamus, very high levels of MAO-B activity have also been found in astrocytes, glia, and the ependymal cells lining all ventricles (Levitt et al., 1982; Westlund et al., 1985). This is significant, because dopamine is known to be released into the extracellular space and is cleared by uptake into astrocytes and glia that surround the synaptic cleft. Though it has not yet been confirmed, MAO-B may play a vital role in degrading dopamine in the astrocytes and glial cells that envelop the synaptic cleft. MAO-A enzyme activity has also been identified in glial cells, but this activity is much lower than that observed for MAO-B.

It has been reported that glial cells show no expression of MAO mRNA (Saura et al., 1996a). Given the high levels of particularly MAO-B enzyme activity, this seems unusual. This may be due to sensitivity limitations of the in situ hybridization technique used in the study by Saura et al., or perhaps MAO protein may be synthesized in neurons and transported to nonneuronal cells. The disparity may also be due to a lower turn-over rate of MAO mRNA in glial cells than in neurons, or it is also possible that glial MAO may differ from neuronal MAO due to the use of an alternate transcript site.

The distribution of these enzymes in itself is curious, since MAO-A is expressed primarily in noradrenergic neurons but shows the highest substrate affinity for serotonin; whereas, MAO$B$ is expressed in serotonergic neurons but primarily oxidizes dopamine and phenylethylamine. It was initially proposed that each enzyme is compartmentalized with its 
preferred substrate in specific regions of the brain; however, evidence gathered to date contradicts this hypothesis (Konradi et al., 1989).

MAO has been proposed to play two vital physiological roles in the brain. First, MAO may facilitate uptake of monoamine neurotransmitters from the synaptic cleft into the neuron by maintaining a low cytosolic concentration of these monoamines in neurons (Fig. 2); and second, MAO may play a role in reducing the effects of neurotransmitters and other amines that gain inappropriate entry into neurons. MAO may act to protect the neuron from stimulation by extraneous amines that may interfere with uptake and storage of the natural neurotransmitters (Richards et al., 1992; Thorpe et al., 1987). These mechanisms may also ensure that dopaminergic neurons use only dopamine for neural transmission, and serotonergic neurons only serotonin.

The role played by MAO in dopaminergic neural transmission has not been clearly defined, and there is some contention over which MAO isoform degrades this important neurotransmitter. The dopaminergic system mediates many vital neurological systems and is involved in mechanisms of reward and addiction (Di Chiara et al., 2004; Pontieri et al., 1996). In vitro studies have confirmed that dopamine is a substrate for both MAO-A and MAO-B, with $\mathrm{K}_{\mathrm{m}}$ values of approximately $240 \mu \mathrm{M}$ and $128 \mu \mathrm{M}$, respectively (Edmondson et al., 2009). While MAO-A preferentially degrades dopamine in the rodent, it is generally accepted that dopamine is metabolized by both enzymes in the human brain (O'Carroll et al., 1983) and that both isoforms will oxidize dopamine in proportion to their enzyme concentrations (Oreland, 1993).

While moderate levels of MAO-A mRNA have been detected in dopaminergic neurons of the substantia nigra and ventral tegmentum in rodents (Jahng et al., 1997), this distribution has yet to be confirmed in human brain. A number of histochemical studies have failed to find MAO activity of either isoform localized within dopaminergic neurons in humans (Konradi et al., 1989; Saura et al., 1996a). Another study detected very small amounts of MAO-A in dopaminergic neurons of the substantia nigra; however, the concentration of MAO-A was so small as to be unlikely to influence dopamine concentrations in these neurons (Westlund et al., 1988).

Because of its localization to the ventricular surfaces, blood vessels and capillaries where the blood brain barrier is known to be located, it has also been suggested that MAO plays an important role in the maintenance of the blood-brain barrier (Konradi et al., 1989; Westlund 
et al., 1988). MAO has been shown to play a significant role in the degradation of dietary amines in peripheral organs, and the high levels of MAO activity in the brain may reflect a mechanism for breaking down potentially harmful amines before they can damage neurons or interfere with neuronal signalling.

\subsubsection{2 - Peripheral Localization}

Extensive study into the peripheral localization of MAO enzymes in a number of animal systems and in humans has found MAO activity in all cell types except erythrocytes. Most tissues show expression of both MAO-A and MAO-B protein and MRNA; however, the relative abundance of each isozyme varies on a tissue to tissue basis (Ramonet et al., 2003; Rodriguez et al., 2001; Sivasubramaniam et al., 2003). For example, human blood platelets and lymphocytes express only MAO-B (Thorpe et al., 1987); whereas, human placenta and intestine express predominantly MAO-A (Grimsby et al., 1990). High expression of both MAOA and B has been observed in the stomach, liver, kidney, and heart (Rodriguez et al., 2001; Saura et al., 1996b) where it is thought the enzyme plays a role in degrading potentially harmful dietary amines (Sandler et al., 1981; Thorpe et al., 1987). This theory is supported by the so-called 'Cheese effect' experienced by patients initially trialing irreversible MAO inhibitors such as tranylcypromine. Tranylcypromine was the first MAO-inhibitor antidepressant used clinically, and while it showed some efficacy as an antidepressant, the irreversible inhibition of both MAO isoforms led to severe hypertensive crisis due to tyramine accumulation following ingestion of tyramine-rich foods (Holschneider \& Shih, 2000; Weyler et al., 1990; Youdim \& Finberg, 1987). This strongly suggests that intestinal MAO plays a vital role in protecting the body against sympathomimetic dietary amines.

Immunocytochemical investigation has also found extensive staining for MAO-A protein in the human placenta, in contrast to MAO-B which showed little activity above baseline (Thorpe et al., 1987). Northern blot analysis has confirmed these high levels of MAO-A mRNA in human placenta, but no MAO-B mRNA was detected (Grimsby et al., 1990). However, immunoprecipitation of the enzyme has provided evidence of very low levels of MAO-B enzyme in human placenta (Thorpe et al., 1987). Placental MAO-A activity is localized to the syncytiotrophoblast layer, between the maternal and fetal circulatory systems, and it has been proposed that these high concentrations of MAO-A serve to protect the fetus from toxic amines in the maternal blood supply. MAO activity may indeed be vital to survival of the fetus 
in mammals, since the MAO inhibitor pargyline causes abortion in both rats and humans when infused into amniotic fluid at high concentrations (Thorpe et al., 1987). Human liver also shows high levels of MAO-A and MAO-B activity. In the liver, MAO detoxifies circulating amines that may be harmful to the brain or that may perturb normal physiology (e.g. sympathomimetic amines) (Thorpe et al., 1987).

Thus, in addition to its vital role in mediating neurotransmitter concentrations in the brain, MAO also appears to be essential in many peripheral organs, providing defense against potentially toxic amine compounds ingested in the diet and produced by normal metabolism (Sandler et al., 1981).

\section{4 - INHIBITION OF MAO BY TOBACCO SMOKE}

Many studies have reported an association between tobacco smoke and reduced MAO activity in the brain and peripheral organs. In vivo and in vitro studies have confirmed that cigarette smoke inhibits MAO activity (Berlin et al., 1995b; J. S. Fowler et al., 2003; Oreland et al., 1981; J. E. Rose et al., 2001), although the mechanism of this inhibition has not yet been elucidated. Chronic exposure to physiological concentrations of nicotine has no effect on the activity of MAO-A or MAO-B in rat brain, suggesting that the inhibition of MAO activity is not nicotine related (Castagnoli et al., 2002). Although nicotine shows some weak inhibition of MAO, this occurs only at concentrations 2000 times those found in chronic heavy smokers and is therefore unlikely to be physiologically relevant (Oreland et al., 1981). Although nicotine itself does not inhibit the action of MAO, a number of other compounds in tobacco smoke show potent inhibitory activity toward MAO-A and B.

The activity of MAO-A and B was investigated in the brain and peripheral organs of human smokers and non-smokers in several comprehensive studies by Fowler et al. (1996a, b; 2000; 2003a, b). Radio-labeled clorgyline was administered to smokers and non-smokers to visualize the activity of MAO-A in the brain by positron emission tomography (PET) scanning (J. S. Fowler et al., 1996b). It was found that smokers showed between $22 \%$ and $38 \%$ less MAO-A activity in some brain regions than non-smokers. The occipital cortex showed the greatest reduction in MAO-A activity with $38 \%$ inhibition, followed by the temporal cortex and cerebellum, each inhibited by $29 \%$. The basal ganglia showed the least inhibition at $22 \%$. The 
average level of MAO-A inhibition over all brain regions in smokers was $28 \%$. Although activity levels of MAO can vary up to 50 -fold between individuals, a consistent and significant reduction in MAO-A activity was observed in smokers compared to non-smokers in the above PET study. This reduction in activity was statistically significant, yet it is uncertain whether this is a physiologically significant reduction. MAO-A inhibitors are used successfully as antidepressants, although estimates of the MAO-A inhibition required for a clinical improvement vary considerably from between $20 \%$ to $80 \%$ (Berlin et al., 1995b). The average inhibition of $28 \%$ due to cigarette smoke is in the low range of these estimates, and Berlin et al. conceded that further studies were required to clarify whether MAO-A inhibition due to chronic cigarette smoke exposure has a physiologically relevant anti-depressant effect.

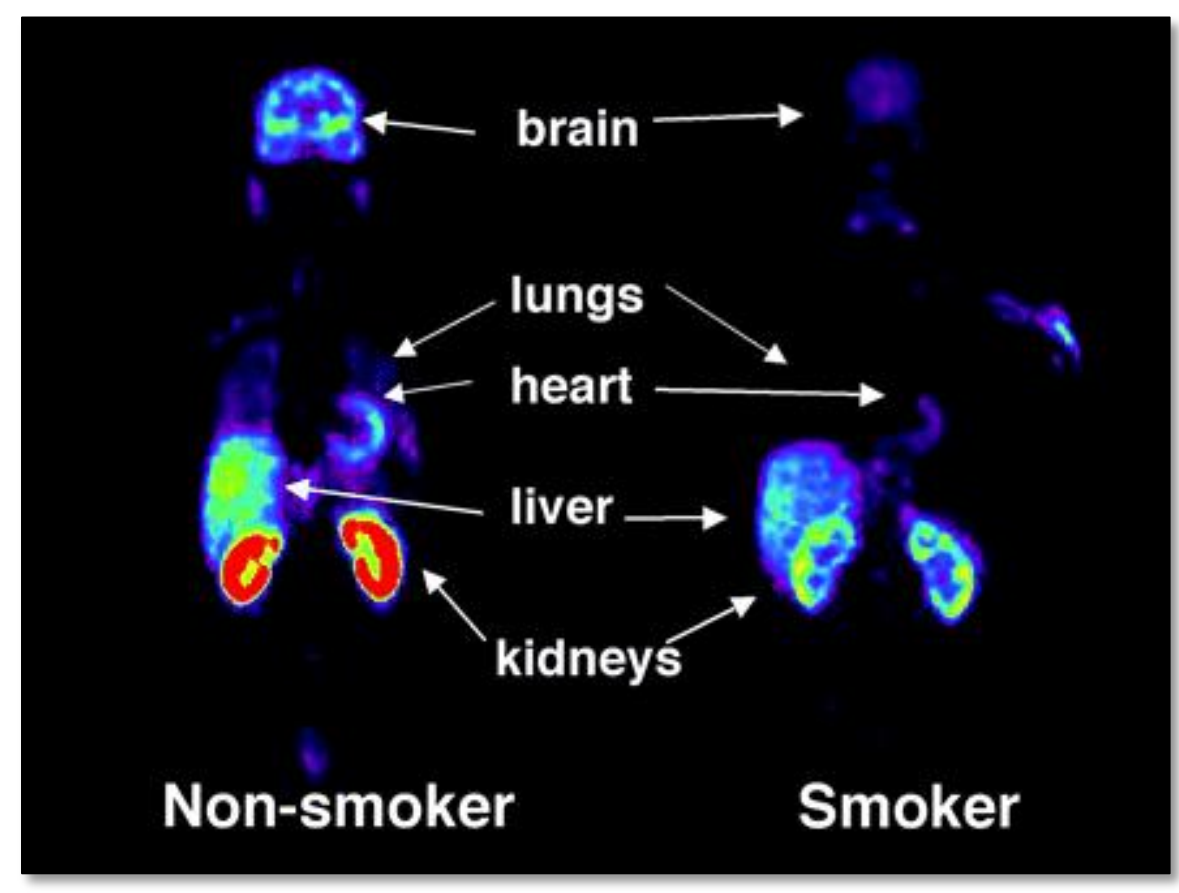

Figure 5: Whole body imaging of radiotracer distribution, bound to MAO-B, in a nonsmoker compared to a smoker. Red is the highest radiotracer concentration on the colour scale.

(Reproduced with permission, from Fowler et al. (2003b) Low monoamine oxidase B in peripheral organs in smokers. Proceedings of the National Academy of Science USA, 100, 11600-11605.)

In another study using PET scanning with radio-labeled deprenyl, MAO-B activity was examined in the brains and peripheral tissues of smokers, non-smokers and ex-smokers (J. S. Fowler et al., 1996a). On average, MAO-B activity was inhibited by $40 \%$ in the brains of chronic smokers compared to non-smokers and ex-smokers. No significant difference in enzyme activity was found between the brains of non-smokers and ex-smokers. From these 
results it was concluded that chronic cigarette smoking causes inhibition of MAO-B in the human brain, but that MAO-B enzyme activity returns to normal levels after smoking cessation. Genetic differences in MAO-B activity were ruled out, since no difference in enzyme activity was observed between non- and ex-smokers. The degree of MAO-B inhibition by cigarette smoke was also measured and compared to the inhibition caused by a therapeutic dose of deprenyl. Deprenyl treatment inhibited MAO-B activity by more than $90 \%$ compared with $40 \%$ MAO-B inhibition observed in the brains of smokers.

In an extension of the above experiment, MAO-B inhibition was assayed in smokers to investigate whether MAO-B levels recovered to the levels seen in non-smokers after overnight abstinence, and whether inhibition could be observed $10 \mathrm{~min}$ after a cigarette (J. S. Fowler et al., 2000). Once again, smokers were found to have an overall $39 \%$ inhibition in MAO-B levels compared to non-smokers. No significant difference was observed between MAO levels in smokers after an 11-hour abstinence or $10 \mathrm{~min}$ after smoking. It was also noted that there was no significant correlation between plasma cotinine (the major metabolite of nicotine) and nicotine concentration, MAO-B inhibition, and the number of cigarettes smoked. The results of this study suggest that the degree of MAO-B inhibition associated with chronic cigarette use is stable over time and is not dependent on the amount of tobacco smoked.

This hypothesis was further tested by Gilbert et al. (2003) in a study that measured platelet MAO-B inhibition in quitting smokers. Blood samples were obtained from smokers in four interviews over the first 31 days of smoking abstinence. Platelet MAO-B levels showed an increase of $22 \%$ above baseline activity after three days of smoking abstinence in quitters relative to active smokers (Gilbert et al., 2003). After 10 days of abstinence the quitting group of participants showed $54 \%$ more MAO-B activity than smokers, and this level of recovery was maintained over days $10-31$ of the experiment. The slow recovery of MAO-B activity suggests that the enzyme is irreversibly inhibited by compounds in cigarette smoke, and that after smoking cessation, MAO levels return to normal via de novo synthesis of the enzyme.

The effect of cigarette smoke was also tested by Fowler et al. (2003b) in peripheral organs, since MAO has important functions in many tissues, and a large number of organs within the body are exposed to compounds in tobacco smoke after inhalation (J. S. Fowler et al., 2003). PET scanning with radio-labeled deprenyl was again used to measure levels of MAO-B in both smokers and non-smokers. It was found that MAO-B activity was significantly reduced in the heart, lungs, kidneys, and spleen of smokers (Fig. 4), with overall inhibition of $45 \%$ in the peripheral organs of smokers relative to non-smokers. 
A recent study on the peripheral activity of MAO-A found that smokers showed a $50 \%$ decrease in lung MAO-A compared to non-smokers (J. S. Fowler et al., 2005). This is a significant change, since MAO-A plays a protective role in the lung by degrading locally released norepinephrine and other circulating amines. Fowler et al. proposed that lung MAOA inhibition may augment nicotine-induced increases in norepinephrine concentration, contributing to some of the peripheral physiological effects of tobacco smoking.

It is not yet known what effect MAO-B inhibition has on the functioning of peripheral organs; however, it is expected that inhibition of these enzymes would have a profound effect on normal function, as seen with the 'Cheese effect' described earlier. Catecholamines are major signalling molecules, playing vital roles in the physiology of the heart, peripheral circulation, and kidneys. In addition, inhibition of MAO in the gut is thought to cause accumulation of tyramine, which has a strong sympathomimetic action.

The long-term consequences of MAO inhibition by cigarette smoke are not known; however, epidemiological studies have shown a strong inverse correlation between cigarette smoking and Parkinson's disease (PD) (Morens et al., 1995). Treatment with the MAO-B inhibitor deprenyl slows the progression of PD and improves many of the symptoms associated with this neurological disorder. This suggests that compounds in cigarette smoke may have a protective effect against the development of PD. Therapeutic MAO inhibitors have proven to be effective in preventing onset of Parkinsonian symptoms in mouse models and are being trialed as human therapeutics to alleviate symptoms in Parkinson's sufferers. Thus, identification of the MAO inhibitory compounds in cigarette smoke may not only aid in the development of more effective smoking cessation therapies but may also lead to more effective treatments for PD.

The evidence gathered to date convincingly suggests that MAO-A and MAO-B are inhibited by compounds in cigarette smoke in both a reversible and irreversible fashion (Table 2). Studies into the significance of this inhibition are currently being undertaken in a number of laboratories, and MAO inhibition could prove to be an important factor in the establishment and reinforcement of tobacco addiction. Identification of the non-nicotine compounds in tobacco smoke that inhibit MAO activity is a complex task and is still under investigation. 


\subsection{1 - MAO Inhibitors in Tobacco Smoke}

Although plasma concentrations of nicotine, cotinine and cyanide and their metabolites are elevated in cigarette smokers, none of these compounds has been linked with any MAO inhibitory action. As previously discussed, nicotine is a poor MAO inhibitor, especially at physiological concentrations (Oreland et al., 1981). A number of studies have identified and characterized several compounds in cigarette smoke that inhibit both MAO-A and MAO-B in a reversible or irreversible manner (Table 2). Characterization of these compounds is important, as their identification will further our understanding of the neurobiology of tobacco addiction and may eventually lead to the development of improved smoking cessation strategies.

In an early study, the MAO inhibitory action of tobacco smoke and tobacco leaf extracts was tested in preparations of rat lung MAO (P. H. Yu \& Boulton, 1987). It was found that unidentified compounds within cigarette smoke and leaf extracts irreversibly inhibited the action of MAO-A and MAO-B. The tobacco smoke extract showed greater inhibitory action on MAO than leaf extracts, and total inactivation of MAO was achieved using high concentrations of tobacco smoke extract. It was deduced from kinetic studies that these compounds displayed a mixed type of inhibition of MAO, that is, that MAO was inhibited both competitively and non-competitively.

More recent investigations by other research groups attempting to identify MAO inhibitors in cigarette smoke have met with some success. Castagnoli et al. (2003) identified 2,3,6trimethyl-1,4-naphthoquinone (TMN) in tobacco smoke, and this compound was found to inhibit both MAO-A and B activity in a mouse model. However, high concentrations of TMN were required to induce this inhibition. While this compound may contribute to some of the effects of tobacco smoke on MAO activity and dopamine concentrations, it is likely that there are also other compounds that inhibit MAO activity in tobacco smoke.

The inhibitory properties of tobacco leaf and smoke extracts in vitro were also investigated in rat mitochondrial preparations (Castagnoli et al., 2002). Extracts of tobacco leaf were found to inhibit MAO-B by $34 \%$; whereas, treatment with tobacco smoke extracts inhibited MAO-B by $93 \%$. Although it is difficult to directly relate a leaf extract to a smoke extract, these results suggest that combustion of tobacco leaf may activate some inert compounds in the tobacco, increasing their capacity to inhibit MAO, or that some MAO inhibitors may be more active in the gaseous phase. This study also identified three inhibitory compounds within cigarette smoke following extraction, fractionation, and electron impact gas chromatography mass 
spectrometry (GC-EIMS) analysis. These compounds included farnesylacetone, and two others not yet fully characterized (Castagnoli et al., 2002). Further fractionation of tobacco smoke components by Khalil et al. (2006) identified farnesol as a significant inhibitor of MAO enzyme activity. Farnesol was found to potently inhibit human liver MAO-B with a $K_{i}$ of 800 $\mathrm{nM}$, but had no activity against MAO-A at concentrations up to $10 \mu \mathrm{M}$ (A. A. Khalil et al., 2006).

2-Naphthylamine is a well-known carcinogen found in cigarette smoke (Hauptmann \& Shih, 2001) that also inhibits brain MAO activity. Mitochondria isolated from mouse brain were pre-treated with 2-naphthylamine before incubation with a MAO-A or B selective substrate. A concentration-dependent inhibition of both MAO-A and B was observed after treatment with this compound. It was also noted that 2-naphthylamine preferentially inhibited MAO-B. The authors conceded that it was uncertain whether 2-naphthylamine was able to cross the bloodbrain barrier to inhibit MAO in the brain, and therefore questioned whether the inhibition induced by this compound was physiologically relevant.

There is convincing evidence that acetaldehyde, one of the major constituents of tobacco smoke, may play a number of pro-addictive roles in the etiology of tobacco dependence (Talhout et al., 2007). Acetaldehyde is produced by the combustion of polysaccharides in tobacco and has been shown to have potent reinforcing properties, particularly on dopamine neurons in the ventral tegmental area. The primary metabolite of ethanol, acetaldehyde, has been studied extensively for its contribution to the reinforcement of ethanol administration, and more recently acetaldehyde has been examined for a potential role in tobacco dependence. While acetaldehyde has been shown to have reinforcing effects on its own, it is also involved in the formation of a number of biologically active compounds produced from the condensation between acetaldehyde and biogenic amines. Significantly, a number of these compounds have been demonstrated to have MAO inhibitory activity.

$\beta$-carbolines are one such group of compounds, formed from the reaction between acetaldehyde and the indoleamines, serotonin, tryptamine, and tryptophan (Talhout et al., 2007). A recent study has identified two of these $\beta$-carbolines, harman and norharman, as potent inhibitors of MAO activity (Herraiz \& Chaparro, 2005). These compounds were isolated from tobacco smoke by HPLC and identified using GC-MS. Norharman was found to competitively inhibit MAO-B in vitro; whereas, harman showed no inhibition of MAO-B. Both $\beta$-carbolines proved to be potent competitive inhibitors of MAO-A activity with $\mathrm{IC}_{50}$ values in the nanomolar range. Harman proved to be the more potent of the two (Herraiz \& Chaparro, 
2005). Following incubation with these inhibitors, enzyme activity could be fully restored, demonstrating that the inhibitory effects of both compounds were reversible.

Another study by Rommelspacher et al. (2002) measured a significant increase in plasma and platelet concentrations of both harman and norharman in smokers following cigarette smoking. This study estimated plasma $t_{1 / 2}$ as $51 \mathrm{~min}$ and $68 \mathrm{~min}$ for norharman and harman respectively, but found $t_{1 / 2}$ in platelets to be much longer, up to 3 hours. As norharman at least is known to be lipophilic, they proposed that cigarette smoking leads to build up of norharman and harman levels in the platelets, and presumably brains of tobacco smokers (Rommelspacher et al., 2002). This study estimated that given the possible cellular accumulation of these compounds and the estimated $t_{1 / 2}$, tobacco contained sufficient harman and norharman for these compounds to reach concentrations that would inhibit MAO-A and MAO-B in the brains of cigarette smokers. The findings of these studies agree with earlier studies that suggested that tobacco smoke extracts contained both reversible and irreversible inhibitors of MAO enzymes.

Tetrahydroisoquinolines (TIQS) are produced from the reaction between acetaldehyde and the catecholamines dopamine, noradrenaline, and adrenaline. Mendez-Alvarez et al. (1997) found that one of these TIQ compounds, 1,2,3,4-tetrahydroisoquinoline (TIQ), (also present in certain foods and beverages) interacts with components of tobacco smoke to form a number of cyanoamine compounds. These cyanoamines were tested for their effects on MAO activity, and many of these adducts showed competitive inhibition of both MAO-A and MAO-B. Of the adducts produced from the interaction between TIQ and tobacco smoke, 1-cyano-TIQ (1CTIQ), $\mathrm{N}$-(1'-cyanoethyl)-TIQ (CETIQ), N-(1'-cyanopropyl)-TIQ (CPTIQ), and N-(1'-cyanobutyl)-TIQ (CBTIQ) were all found to reversibly inhibit MAO activity. It was noted, however, that these cyanoamines were comparatively weak inhibitors of MAO activity and were more potent inhibitors of MAO-B than A. Based on these results, this group proposed that the MAO inhibitory activity of cigarette smoke was not a direct result of inhibitory compounds within the smoke stream but may be due to interactions between tobacco smoke constituents and endogenous or exogenous compounds in the brain that are activated to become MAO inhibitors (Mendez-Alvarez et al., 1997a). However, cigarette smoke is known to irreversibly inhibit the activity of MAO, and therefore the reversible inhibition by cyanoamine adducts observed in this study cannot fully account for all the MAO inhibition reported for cigarette smoke. It is possible that MAO inhibitors are formed as pyrolysis products of the combustion of tobacco plant matter. To date no studies have investigated the MAO inhibitory properties 
of other plants smoked by humans. Some early studies have reported cannabis leaf extract is capable of significantly inhibiting MAO; however, these studies utilized unburnt leaf extracts (Schurr et al., 1978; Schurr \& Rigor, 1984).

Salsolinol is another such TIQ compound, produced from the condensation of acetaldehyde and dopamine (Talhout et al., 2007). While salsolinol has been identified as a MAO-A and MAO-B inhibitor in the micromolar range, the maximum possible concentration of salsolinol produced from smoke-derived acetaldehyde is only $20 \mathrm{nM}$, and this is not high enough to significantly inhibit the activity of MAO enzymes. Interestingly, salsolinol has also been found to be a significant reinforcer in rodent self-administration studies, and further investigation indicated that salsolinol exerts these effects through the $\mu$ opioid receptor (MOR) but by an unknown mechanism (Matsuzawa et al., 2000). As recent evidence suggests a role for MOR in tobacco addiction, salsolinol may influence the mechanisms of tobacco dependence through its actions on MOR.

Although a number of different compounds in tobacco smoke have been identified as having MAO inhibitory properties (Table 2), it would appear that these compounds cannot fully explain the inhibition observed in the human brain and peripheral tissues associated with cigarette smoking. Tobacco smoke inhibits both isozymes of MAO, and studies indicate this happens in both a reversible and irreversible fashion (P. H. Yu \& Boulton, 1987), implying that there are multiple compounds within cigarette smoke that are inhibitory to MAO. Identifying and characterizing these compounds is an important goal of future research as this may lead to more effective smoking cessation strategies and will contribute to a better understanding of the neurobiology of tobacco addiction. 
Table 2: Monoamine oxidase inhibitors identified in tobacco smoke

\begin{tabular}{|c|c|c|c|}
\hline Compound & Inhibition & Reference & Structure \\
\hline $\begin{array}{l}\text { Trans-trans- } \\
\text { Farnesol }\end{array}$ & $\begin{array}{l}\text { Selective inhibitor of MAO-B } \\
\mathrm{K}_{\mathrm{i}}=0.8 \mu \mathrm{M}\end{array}$ & (A. Khalil et al., 2006) & \\
\hline Farnesylacetone & & (Castagnoli et al., 2002) & \\
\hline $\begin{array}{l}\text { 2,3,6-trimethyl-1,4- } \\
\text { naphthoquinone }\end{array}$ & Inhibits MAO-A and B. & (Castagnoli et al., 2002) & \\
\hline TIQ adducts & $\begin{array}{l}\text { Competitively inhibit MAO-A and } B \text { at } \mu \mathrm{M} \\
\text { conc. Reversible inhibition. }\end{array}$ & $\begin{array}{l}\text { (Mendez-Alvarez et al., } \\
\text { 1997a) }\end{array}$ & \\
\hline 2-Naphthylamine & $\begin{array}{l}\text { Potent inhibition of MAO-B. } \\
\mathrm{K}_{\mathrm{i}}=40 \mu \mathrm{M}\end{array}$ & (Hauptmann \& Shih, 2001) & \\
\hline Harman & $\begin{array}{l}\text { Competitive inhibitor of MAO-A } \\
\mathrm{IC}_{50}=0.34 \mu \mathrm{M}\end{array}$ & (Herraiz \& Chaparro, 2005) & \\
\hline Norharman & $\begin{array}{l}\text { Competitive inhibitor of MAO-A and B. } \\
\text { MAO-A IC } C_{50}=6.5 \mu \mathrm{M} \\
\text { MAO-B IC } C_{50}=4.7 \mu \mathrm{M}\end{array}$ & (Herraiz \& Chaparro, 2005) & \\
\hline
\end{tabular}




\subsection{2 - MAO Inhibitors in Smoking Cessation Therapy}

The majority of cigarette smokers express a desire to quit smoking, but few smokers are successful in their attempts to quit, and most quitters show a high rate of relapse. Smokers who have tried repeatedly to quit and failed commonly turn to pharmacotherapy for assistance. At present pharmacotherapy for treating tobacco addiction is relatively limited, and these treatments predominantly involve different forms of nicotine replacement therapy (NRT). NRT is available as a gum, transdermal patch, nasal spray, or inhaler. These products are designed to pharmacologically replace the nicotine derived from cigarette smoke while the smoker breaks the behavioural cues which accompany smoking. These forms of NRT show differing levels of efficacy; however, their efficacy is thought to be limited by the mechanism of nicotine delivery. Nicotine inhaled in a bolus of cigarette smoke can reach the brain in approximately $10 \mathrm{sec}$. Drug delivery speed is thought to be a crucial factor in the effectiveness of NRT, yet currently no form of NRT can match the delivery speed of the cigarette, nor the rapid pharmacological effect of smoke administration. Most smokers report NRT to be much less satisfying than cigarette smoke.

In the United States, bupropion is presently the only non-nicotine drug approved for smoking cessation treatment by the FDA and is marketed under the name 'Zyban'. Bupropion is administered to the smoker as a sustained-release $300 \mathrm{mg}$ per day tablet, and therapy is started 7 days prior to quitting (George \& O'Malley, 2004). Bupropion is thought to inhibit dopamine and noradrenaline transport and reuptake by competing with neuronal transporters (George \& O'Malley, 2004; Xue \& Domino, 2007) and thus increase synaptic dopamine concentrations. The efficacy of bupropion as a smoking cessation aid has been demonstrated in clinical laboratory studies; however, it is expensive and only shows limited success in many smokers.

The lack of success with current pharmacological treatments indicates that the development of more effective strategies to treat nicotine dependence is required. MAO inhibitors administered alone or as adjunct therapy with nicotine replacement regimes are being trialed as aids to smoking cessation (Berlin et al., 1995a; George et al., 2003). Since tobacco smoke irreversibly inhibits MAO, therapeutic administration of MAO inhibitors may assist smoking cessation by mimicking the effect of cigarette smoke on MAO, thus, diminishing the severity of withdrawal symptoms.

Moclobemide, which has previously been used to treat depression, is a reversible inhibitor of MAO-A. It is currently being trialed alone and in conjunction with NRT in dependent smokers 
to assist quitting. In a randomized double-blind study, moclobemide was administered to heavily dependent smokers to assist with smoking cessation (Berlin et al., 1995a). Moclobemide treatment was maintained for three months after the quit day, and it was found that $25 \%$ of dependent smokers using moclobemide reported that they remained abstinent after a year compared with $16 \%$ abstinence in a group of smokers who received placebo (Berlin et al., 1995a). This abstinence rate did not drop significantly, even after moclobemide treatment was stopped. It was proposed that the effectiveness of moclobemide in assisting smoking cessation was due to its ability to manage withdrawal symptoms, including depression and dysphoria.

MAO-B inhibitors have also been trialed as smoking cessation aids, including selegiline hydrochloride (deprenyl), an irreversible MAO-B inhibitor that has been used in the treatment of PD and Alzheimer's disease (Sano et al., 1997). In a double-blind placebo controlled trial, selegiline was found to increase smoking cessation success rates at least as effectively as NRT (George et al., 2003). Selegiline was administered to dependent smokers attempting to quit for 2 weeks prior and 6 weeks after their nominated quit date. Smoking abstinence after 6 months was reported as $20 \%$ for participants treated with selegiline and only $5 \%$ for those receiving placebo. The authors reported that while the rates of smoking cessation were lower than those seen in trials of bupropion, they were comparable to the results seen with NRT.

Additionally, a randomized double-blind study trialing selegiline in conjunction with NRT patches found that smoking cessation rates after one year in the group that had received selegiline were double the cessation rates seen in the group receiving NRT and placebo (Biberman et al., 2003). Continued abstinence in the group receiving NRT and selegiline and the group receiving NRT and placebo were $25 \%$ and $11 \%$, respectively. Although this difference was not found to be statistically significant, it showed a trend that may be exploited for future smoking cessation therapies. Administration of MAO inhibitors in conjunction with NRT could dramatically increase smoking quit rates once an appropriate dosage and treatment regimen is established. 


\section{5 - INTRODUCTION TO THE MU OPIOID RECEPTOR (MOR)}

\subsection{1-General Introduction}

Research into the mode of action of morphine in the early 1970's led to the discovery and classification of endogenous opioid receptors in the central nervous system. These receptors were classified according to their pharmacological properties with respect to morphine, nalorphine, and a number of other opiate compounds, and they were termed the $\mu, \delta$, and $\mathrm{k}$ opioid receptors. These receptors were later found to have intrinsic roles binding endogenous opioid ligands, identified as the enkephalins, endorphins, and endomorphins. These endogenous opioids and the $\mu, \delta$, and $\mathrm{k}$ opioid receptors are expressed throughout the central nervous system. The endogenous opioid system plays a number of crucial roles in regulating mood, memory, learning, analgesia, the immune system, and autonomic functions such as gastrointestinal mobility, respiration, and thermoregulation (Kieffer, 1999; Mansour et al., 1995). Significantly, the endogenous opioidergic system, and MOR in particular, has long been associated with mechanisms of addiction to drugs of abuse, including morphine, and cocaine. Recent evidence also convincingly suggests an indirect role for MOR and the opioid system in the rewarding effects of nicotine and tobacco.

\subsection{2 - Structure, Function, \& Localization}

MOR is expressed on the cell surface of neurons throughout the CNS. Significantly, MOR is widely expressed in areas of the brain associated with addiction and reward pathways, including the mesolimbic dopamine system (Corrigall et al., 2000; Mansour et al., 1995) discussed in detail in section 1.2 of this thesis. In these regions, MOR expression has been identified pre- and post-synaptically on glutamatergic, GABAergic, cholinergic and dopaminergic neurons (Britt \& McGehee, 2008). MOR has also been found in high density in the brainstem, where it is involved in modulation of physiological functions such as respiratory rhythm and antinociception.

Analysis of the cloned MOR-1 receptor has indicated that MOR is a membrane-associated glycoprotein belonging to the class A Rhodopsin G-protein coupled receptor superfamily (Zaki et al., 1996). The receptor is comprised of seven transmembrane domains linked by three intracellular and three extracellular loops, and conforms to the typical 3-D structure seen in the Rhodopsin family. That is, the seven transmembrane domains are arranged anti-clockwise to form a tight bundle of seven helices. The receptor also comprises an extracellular amino 
terminus that exhibits five putative sites of $\mathrm{N}$-linked glycosylation and an intracellular $\mathrm{C}$ terminus. The cloned human MOR-1 receptor cDNA predicts a protein of 400 amino acids with a molecular weight of approximately $58 \mathrm{kDa}$ before post-translational modification (Minami \& Satoh, 1995). MOR shares approximately $60 \%$ amino acid sequence identity with the $\delta$ and $\mathrm{k}$ receptors, being most conserved through the membrane-spanning regions; while, the extracellular regions show the most divergence (Waldhoer et al., 2004). In humans, the MOR gene, Oprm, is located on the long arm of chromosome 6 . The gene comprises 8 introns, that are alternatively spliced to produce at least 10 identified splice variants (Pan et al., 2005). The longest splice variant, MOR-1, encodes the main MOR isoform. All the splice variants described to date contain exons 1, 2 and 3 which encode the amino-terminus and all seven transmembrane domains. As the amino-terminus and transmembrane domains comprise the ligand binding pocket of MOR, the splice variants show similar ligand specificities and binding affinities (Pan et al., 2005).

Opioid substrates bind to MOR on the extracellular side, causing receptor activation and coupling with G-proteins on the intracellular surface of the plasma membrane. Activated Gproteins regulate the actions of a number of second messenger intracellular signaling molecules, including adenylyl cyclase, phosphatidylinositol 3-kinase, MAP kinase, and calcium channels (Christoffers et al., 2003; Law et al., 2000). Acute exposure to MOR agonists causes receptor activation, followed by receptor desensitization, the latter caused by uncoupling of the receptor to the G-protein by receptor phosphorylation (Waldhoer et al., 2004). Chronic stimulation of MOR by opioid agonists generally results in internalization of the receptor via clathrin-coated pits and proteolytic degradation, causing an observed down-regulation of MOR. Binding of opioid peptides to MOR results in a number of physiological effects, including antinociception, euphoria, respiratory depression, and inhibition of gastrointestinal mobility. The binding of exogenous opioid receptor agonists, such as morphine, induces these same effects, with constipation and respiratory depression being well documented side effects of opiate drug abuse.

The natural substrates for the opioid receptors are families of opioid peptides, the enkephalins, dynorphins, endorphins, and endomorphins. The most likely natural ligands for MOR are $\beta$-endorphin, and endomorphin which show high affinity for the receptor (Kieffer, 1999; Waldhoer et al., 2004; Zadina et al., 1997). Endomorphin is found in high concentrations in the amygdala and nucleus accumbens (Fichna et al., 2007); while, $\beta$ endorphin neurons are located mainly in the pituitary gland and hypothalamus and project to 
the ventral tegmental area and nucleus accumbens (Roth-Deri et al., 2008). As MOR agonists, these peptides display behavioural and physiological effects similar to morphine. They are readily self-administered and elicit place preference when used in animal experiments (RothDeri et al., 2008). Many drugs of dependence have been shown to induce release of $\beta$ endorphin following administration. It is through mediating the actions of these opioid peptides that MOR is presumed to influence motivation and reward pathways and the mechanisms of drug dependence.

\section{$\underline{1.5 .3-M O R \text { and Tobacco }}$}

Several studies have reported associations between the endogenous opioid system and nicotine; however, the mechanisms underlying this association are not yet well characterized (Walters et al., 2005). An opioid link in tobacco addiction was first postulated when it was noted that the MOR antagonist naloxone reduced the smoking behavior and tobacco craving in human smokers (Karras \& Kane, 1980). Wewers et al. (1998) also reported that human smokers receiving a dose of the MOR antagonist naltrexone smoked less cigarettes over a 3 day period than a group receiving placebo treatment (Wewers et al., 1998). Animal studies have reported that naloxone blocks the antinociceptive effect of nicotine observed in mice and also attenuates nicotine withdrawal symptoms such as chattering teeth, gasping, and tremors in nicotine-dependent animals. Naloxonazine, another MOR antagonist, has also been shown to block nicotine-induced antinociception in mice (Simons et al., 2005). These results strongly suggest the involvement of MOR in mediating the antinociceptive and other physiological effects of nicotine that have been reported previously.

Extensive pharmacological and molecular studies have been conducted into the function and influence of MOR and the opioid system, which was summarized in section 1.5.2. These studies have confirmed that the endogenous opioid system influences the reinforcement of morphine, heroin, and many other drugs of abuse (Contet et al., 2004). Importantly, it has been demonstrated that reinforcement and reward of ethanol, cannabis, and nicotine is also abolished or significantly attenuated in knock-out mice lacking MOR. Studies utilizing MOR knock-out mice have reported that these mutant mice show no increase in pain tolerance following administration of nicotine at doses that induce antinociception in controls. This confirms the previous finding that MOR is linked to nicotine antinociception in animals. MOR knock-out mice also show no conditioned place preference to nicotine reward, nor do they 
learn to self-administer nicotine (Berrendero et al., 2002). This suggests that MOR is required not only for nicotine antinociception, but also to mediate the rewarding properties of nicotine. The mechanism by which MOR influences nicotine reward has not been elucidated, but a role for endogenous opioid peptides in this process has been proposed.

Tanda \& Di Chiara (1998) demonstrated that MOR activation in the ventral tegmental area is required for nicotine-induced dopamine overflow in the nucleus accumbens. They showed a significant increase in dopamine release in the accumbens following administration of nicotine in rats. This dopamine increase was abolished in rats pre-treated with the selective MOR antagonist naloxonazine, applied directly to the ventral tegmental area (Tanda \& Di Chiara, 1998). This study hypothesized that endogenous $\mu$-opioid tone modulates the activation of dopamine under basal conditions, and that drugs of abuse increase opioid tone, leading to disinhibition of mesolimbic neurons and resulting in dopamine release. The suggestion that MOR may be modulating the various effects of nicotine by mediating the action of $\beta$ endorphin and other opioid peptides is an appealing one, as nicotine has been shown to evoke $\beta$-endorphin release in humans and laboratory animals. B-endorphin is an agonist for MOR and shares a number of properties in common with other MOR agonists discussed here. That is, $\beta$-endorphin is readily self-administered by laboratory animals and induces a conditioned place preference. In humans, a significant increase in plasma $\beta$-endorphin concentrations was recorded following nicotine treatment (Pomerleau et al., 1983b). Davenport et al. (1990) provided convincing evidence that nicotine administration caused endogenous MOR peptide release. Mice were treated with nicotine before receiving a dose of $\beta$-funaltrexamine, an irreversible MOR antagonist. Nicotine pre-treatment attenuated $\beta$ funaltrexamine antagonism, suggesting that nicotine administration had caused the release of endogenous opioid peptides that had bound and activated MOR and blocked $\beta$ funaltrexamine binding (Davenport et al., 1990). Additionally, Boyadjieva \& Sarkar (1997) demonstrated the secretion of $\beta$-endorphin from primary cultured rat hypothalamic neurons stimulated with nicotine. Acute nicotine administration induced increased $\beta$-endorphin release of up to $500 \%$ of control. However; this effect showed rapid desensitization to chronic nicotine stimulation, as $\beta$-endorphin release returned to baseline levels after 24 hours stimulation (Boyadjieva \& Sarkar, 1997). Spanagel et al. (1990) showed that $\beta$-endorphin administration caused an increase in dopamine release and metabolism in the nucleus accumbens of rats in a dose-dependent manner. This effect was abolished in rats pre-treated with the MOR specific antagonist D-Pen-Cys-Tyr-D-Trp-Orn-Thr-Pen-Thr- $\mathrm{NH}_{2}$ (CTOP). Thus, they confirmed that $\beta$-endorphin induces dopamine overflow in the nucleus accumbens and 
exerts this effect through MOR (Spanagel et al., 1990). To summarise, MOR is thought to be involved in nicotine reward through its role in mediating the actions of endogenous opioid peptides. Nicotine binding to nAChRs causes release of $\beta$-endorphin and other opioid peptides. These bind to MOR located on neurons in the VTA, which in turn leads to dopamine release in the nucleus accumbens.

Non-nicotine compounds in tobacco smoke may also influence the brain reward pathways mediated by MOR. Salsolinol is a condensation product of the reaction between dopamine and acetaldehyde, large quantities of which are found in tobacco smoke. It induces naloxonesensitive antinociception in rodents and shows rewarding properties in conditioned place preference studies and self-administration paradigms (Matsuzawa et al., 2000). It also induces an increase in locomotor activity, thought to be associated with the rewarding properties of drugs of abuse. Salsolinol has been studied as a potential neurochemical factor in alcoholism and has been reported to activate the opioid system, although its potential role in tobacco dependence has yet to be determined (Talhout et al., 2007).

The evidence reviewed above strongly suggests a critical role for MOR and endogenous opioid signalling in mediating the rewarding and reinforcing properties of nicotine. There is also reason to believe that the non-nicotine components of tobacco smoke may modulate the influence of the opioid system on nicotine reward and reinforcement. However, the details and mechanisms of action of the endogenous opioid system and the role of MOR in tobacco dependence have yet to be elucidated, and more research in this area is warranted.

\section{6- AIMS}

Nicotine has long been held as the principal neurobiologically active compound in tobacco smoke and has been studied extensively as the primary biological influence on tobacco addiction. However, there is a growing body of evidence that suggests that other compounds within tobacco smoke may also influence addiction in direct and indirect ways. Changes in MAO enzyme activity have been linked to tobacco addiction. Cigarette smoking reduces MAO activity throughout the body, in particular in regions of the brain associated with rewardoriented behaviors. Many studies are currently attempting to identify MAO inhibitory 
compounds in tobacco smoke, and these MAO inhibitors may provide promising options for a new generation of pharmacotherapies for tobacco dependence. Ongoing research into the role of MAO in the CNS and the influence of monoamine oxidase inhibitors is required for a more complete understanding of the mechanisms of tobacco dependence. Recent evidence also suggests the indirect involvement of the endogenous opioid system in mediating the rewarding properties of tobacco, potentially through the action of MOR. Further research is required to elucidate the role that endogenous opioid peptides and MOR play following exposure to tobacco smoke.

The present study aimed to investigate, compare, and contrast the influence of tobacco and the non-nicotine components of tobacco with nicotine on the function and gene expression of several neurobiologically relevant drug targets in neuronal and glial cell lines in an attempt to identify non-nicotine effects on these cells that may influence tobacco addiction. In particular, this research hoped to identify changes in monoamine oxidase enzyme activity following in vitro exposure to nicotine, standard tobacco particulates, and denicotinized tobacco particulates after several different treatment regimens. It was proposed that a detailed knowledge of the differences in MAO response to nicotine, tobacco extracts and denicotinized tobacco extracts may help elucidate the role that MAO inhibitors play in tobacco addiction.

MAO gene expression in response to nicotine and tobacco exposures was also investigated in vitro. Much research has been conducted into the effects of tobacco smoke on the activity of MAO-A and MAO-B, but few studies have investigated gene expression following exposure to these compounds. This project investigated whether there was an effect on MAO-A and MAO-B gene expression in vitro following exposure to nicotine and tobacco particulate extracts. Additionally, this study aimed to investigate differences between neuronal cells and glial cells in their responses to nicotine and tobacco exposure. Primarily, differences in MAO activity and gene expression between neuronal and glial cells were examined. Because glial cells are known to display strong MAO activity in vivo, and recent evidence suggests a putative role for glia in establishment of drug dependence, this study hoped to determine whether an in vitro model of glial cell function (U-118 MG) responded differently to a neuronal cell model (SH-SY5Y) when exposed to tobacco extracts.

Recent evidence confirmed that opioid mechanisms play a role in the neuronal systems mediating reward and reinforcement. Research has also suggested involvement of the endogenous opioid system and MOR in contributing to the addictive properties of tobacco 
smoke. The present study intended to characterize gene expression of MOR in response to nicotine and tobacco extract exposure with the aim of providing insights into the role of MOR in establishing and maintaining tobacco addiction.

Ultimately, the aims of this thesis were to examine the non-nicotine-mediated effects of tobacco smoke on important neural pathways implicated in reward and reinforcement and to determine how these may pertain to tobacco addiction. It was thought that a deeper understanding of the non-nicotine effects of tobacco smoke will in turn lead to promising developments and improvements in tobacco cessation therapies. 


\section{Chapter Two: General Methods}

\section{1-Cell CULture}

This thesis describes studied designed to examine the effects of tobacco compounds in vitro. The majority of experiments that make up the body of this thesis were conducted on cultured human cells, which were selected as models of human neuronal and glial cell functioning. The methods used to culture and maintain these cell lines are outlined below.

\subsection{1 - SH-SY5Y Human Neuroblastoma}

SH-SY5Y cells are a human neuroblastoma cell sub-line cloned from the SK-N-SH neuroblastoma cell line. The SK-N-SH neuroblastomacell line was established in 1970 from a metastatic bone tumour isolated from a 4-year old female patient. The SH-SY5Y cells grow as a mixture of adherent and free-floating cells, with an epithelial morphology. Adherent cells grow in clusters of neuroblastic cells which are stellate in appearance, and display short, fine processes.

The SH-SY5Y cell line was selected for this study as it is a commonly used model of neuronal cell behavior and physiology. Many studies have been conducted previously examining the response of SH-SY5Y cells to nicotine and tobacco extracts (Ambrose et al., 2007; Dunckley \& Lukas, 2006; Sokolova et al., 2005), and using this cell line as the primary experimental organism for this study allows direct comparison with these previously published experiments. SH-SY5Y cells were not treated with retinoic acid in this study, and undifferentiated cells were used for all experiments.

Frozen stocks of SH-SY5Y were obtained from the American Type Tissue Collection facility (Catalogue no. CRL 2266). These cells were grown in Roswell Park Memorial Institute 1640 medium (RPMI-1640, GIBCO, Invitrogen, CA, USA) supplemented with 10\% heat-inactivated fetal calf serum (FCS, v/v), $50 \mathrm{U} / \mathrm{mL}$ penicillin $\mathrm{G}$, and $50 \mu \mathrm{g} / \mathrm{mL}$ streptomycin sulphate (all sourced from GibcoBRL, Invitrogen Life Sciences Inc. CA, USA). Culture and passage conditions are described in detail in section 2.1.3. 
(a)

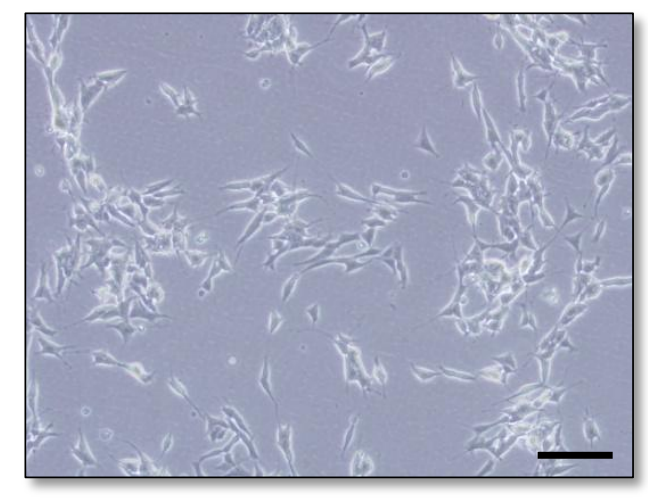

(b)

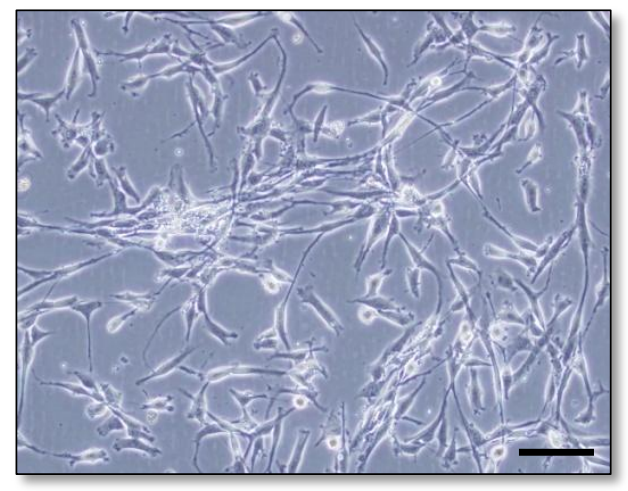

Figure 6: (a) SH-SY5Y human neuroblastoma cells grown in culture, at low density. Viewed at 400x magnification. (b) U-118 MG human glioma cells grown at moderate density. Viewed at 400x magnification. Scale bars represent $100 \mu \mathrm{m}$.

\subsection{2 - U-118 MG Human Glioma}

U-118 MG cells are derived from a human glioblastoma cell line obtained from a malignant primary glioma isolated from the brain of a 50 year old male patient. The cells grow densely and adherently with a mixed morphology, and display long, fine processes.

Recently, research has been conducted toward understanding the role of glial cells in brain function, addiction mechanisms and the impact of drugs of abuse. To date, most studies of the effects of nicotine and tobacco on glial cells have examined their in vivo activity. A human in vitro model of glial cell functioning was sought to compare with the neuronal SH-SY5Y cells in this study. The U-118 MG cell line was chosen from the American Type Tissue Collection facility (Catalogue no. HTB-15) as it showed only minor cytogenetic abnormalities in comparison with other glioma cell lines, and could be cultured under the same conditions as the SH-SY5Y cell line. Additionally, studies had reported that the U-118 MG cell line expresses acidic glial fibrillary protein (Feindt et al., 1995) - an accepted marker for glial and astrocytic cells - and so it was thought that U-118 MG may provide a useful in vitro model of glial cell function to investigate the effects of tobacco and nicotine on monoamine oxidase enzymes and opioid receptors.

U-118 MG cells were cultured in RPMI-1640 medium, supplemented with $10 \%$ heatinactivated FCS, $50 \mathrm{U} / \mathrm{mL}$ penicillin, and $50 \mu \mathrm{g} / \mathrm{mL}$ streptomycin sulphate, as described below. 


\section{$\underline{2.1 .3-C u l t u r e ~ C o n d i t i o n s}$}

Cells frozen in RPMI-1640 growth medium containing 10\% DMSO (Sigma-Aldrich Co. St Louis, USA) were stored in liquid nitrogen. When required, frozen cells were removed from liquid nitrogen storage and thawed rapidly in a $37^{\circ} \mathrm{C}$ water bath. Thawed cells were then added to 5 $\mathrm{mL}$ of RPMI-1640 culture medium (GIBCO, Invitrogen Ltd, CA, USA), supplemented with $10 \%$ $(\mathrm{v} / \mathrm{v})$ heat-inactivated fetal calf serum (FCS), 50 units $/ \mathrm{mL}$ penicillin $\mathrm{G}$ sodium, and $50 \mu \mathrm{g} / \mathrm{mL}$ streptomycin sulphate (all sourced from GibcoBRL, Invitrogen Life Sciences Inc.). Cells suspended in media were then centrifuged at room temperature (RT) for $5 \mathrm{~min}$ at $300 \mathrm{xg}$ using a Sigma 3-16 benchtop centrifuge. The medium supernatant was removed from the cell pellet by aspiration, and the pellet was resuspended in fresh pre-warmed feeding medium.

SH-SY5Y and U-118 MG cells were seeded initially in $50 \mathrm{~mL}$ filter-capped cell culture flasks (Greiner Bio-one) and grown directly on the polystyrene inner surface of the flasks. SH-SY5Y cells displayed a doubling time of approximately 24 hours, while U-118 MG cells multiplied slightly faster. The U-118 MG cells could also grow to much higher densities than SH-SY5Y. When grown at high density U-118 cells displayed a long, thin morphology, but growing at high density was avoided, as the cells became difficult to detach from the polystyrene substrate. When cell numbers had increased to approximately $90 \%$ confluence, cultures were passaged, and the cells seeded in larger $250 \mathrm{~mL}$ or $650 \mathrm{~mL}$ filter-capped polystyrene culture flasks (Greiner Bio-one). Flasks of cells were incubated at $37^{\circ} \mathrm{C}$ in a humidified, $5 \% \mathrm{CO}_{2}$ atmosphere in a Heraeus incubator (model HeraCell), and the cells were passaged every 3-7 days, or when they reached $90 \%$ confluence. The RPMI 1640 medium was replaced in flasks of growing cells every 5 days, or as required.

\subsection{4-Cell Passaging}

Cell cultures were fed and passaged in a horizontal laminar flow cabinet (Biological safety cabinet class II, AES Environmental Inc.). Before use, the laminar flow cabinet was sterilized with UV light for $15 \mathrm{~min}$, and the inner surfaces wiped with 70\% ethanol. Culture medium was removed from flasks of near-confluent cells by aspiration, and the cells were rinsed with 250 $\mu \mathrm{L}$ of $0.05 \%$ trypsin/0.53 mM EDTA solution (Invitrogen Life Sciences, Ltd. CA, USA) for $30 \mathrm{sec}$. The trypsin solution was removed by aspiration, and a further $450 \mu \mathrm{L}$ of fresh trypsin solution was added to the culture flasks. Cultures were incubated with the trypsin solution for $3-10$ min, or until most cells had detached from the flask surface. The inner flask surface was then 
rinsed with $5 \mathrm{~mL}$ of fresh, pre-warmed medium, and any remaining adherent cells were detached by tituration with a pipette, or very gentle agitation with a plastic cell scraper (Greiner Bio-one). The cell suspension was then transferred into a $15 \mathrm{~mL}$ polystyrene centrifuge tube (Greiner Bio-one, GmbH. Germany) and centrifuged at $300 \times \mathrm{g}$ in a Sigma 3-16 benchtop centrifuge for $5 \mathrm{~min}$ to pellet the cells. The medium supernatant was removed by aspiration, and the cell pellet resuspended in fresh pre-warmed feeding medium. Cells were then reseeded in sterile $600 \mathrm{~mL}$ polystyrene culture flasks at a ratio of approximately 1:5 $-1: 8$ dilution.

\section{2 - TOBACCO PARTICULATE EXPOSURES}

Cultured SH-SY5Y and U-118 MG cells were exposed to tobacco particulate extracts over a number of different treatment regimens. These specific treatment regimens will be discussed in detail in the experimental chapters of this thesis, but the general methods used for the preparation of the TPM and exposure of the cells are detailed below.

\subsection{1 - Tobacco Particulate Matter Sample Collection}

Several different tobacco types and brands were selected for analysis in this study. Tobacco particulate matter extracts from these cigarette and loose tobacco samples were kindly supplied by the Population and Environmental Health group at Environmental Science and Research (ESR), Kenepuru Science Centre, Porirua, New Zealand.

The majority of the experiments in this thesis compare the effects of two brands of cigarette. These are Holiday Special Filter ${ }^{\circledR}$ - a leading brand of cigarette sold in New Zealand and Quest ${ }^{\circledR}$ 3 Nicotine-Free cigarettes (Vector Tobacco Inc. NC, USA) - marketed as a low-nicotine alternative to tobacco in several states of the US. Holiday ${ }^{\circledR}$ brand cigarettes yield less than 1.2 $\mathrm{mg}$ nicotine and less than $16 \mathrm{mg}$ of tar in each cigarette and the Quest ${ }^{\circledR}$ cigarettes yield no more than $0.05 \mathrm{mg}$ of nicotine and $10 \mathrm{mg}$ tar per cigarette, as reported on the cardboard cigarette packets.

The collection and extraction of all tobacco particulate samples was conducted by ESR Kenepuru Science Centre as follows. 
Cigarette and tobacco samples were sent to Labstat International, Inc. (Kitchener, Canada) where they were burnt and the smoke collected and extracted by smoking machines. The total particulate matter (TPM), which includes nicotine, represents the solid combustion products of tobacco that are inhaled by a smoker. This TPM was collected onto $\varnothing 92 \mathrm{~mm}$ Cambridge paper filter pads using the ISO 3308 method (Ambrose et al., 2007). Each filter typically comprised the TPM collected from twenty cigarettes, and was accurately weighed before and after TPM collection. This data was used by Labstat to report on the amount of tar collected on each filter. The filters containing the TPM were sent back to the ESR Kenepuru Science Centre and stored at $-20^{\circ} \mathrm{C}$ until extraction.

To extract the TPM from the Cambridge filters, the paper filters were cut into quarters and placed in glass extraction tubes. Absolute ethanol was added $(4 \mathrm{~mL})$ to each filter, and the tubes were then vortexed and sonicated to allow thorough penetration of the filter paper by the ethanol. The solvent was decanted into a sterile $15 \mathrm{~mL}$ polypropylene reaction tube (Greiner Bio-one, GmbH. Germany), and this process was repeated a further 5 times, using 2 $\mathrm{mL}$ aliquots of ethanol. Ethanol was chosen as the extraction solvent to allow capture of as many volatile compounds from the tar as possible. The solvent fractions were pooled and assayed for nicotine concentration by ESR Kenepuru, using an Agilent 5973N gas chromatograph. An HP-5MS (30 m, $0.32 \mathrm{~mm}$ I.D., $0.25 \mathrm{~mm}$ film thickness) gas chromatograph column was used, and equipped with a post-column split to a mass spectrometer and nitrogen phosphorous detector. The mass spectrometer confirmed the presence of nicotine and the nitrogen phosphorous detector was used for quantitation. The nicotine concentration of the Holiday ${ }^{\circledR}$ Special Filter TPM (denoted HTPM) was measured to be $1.058 \mathrm{mg} / \mathrm{mL}(6.523 \mathrm{mM})$; and the Quest ${ }^{\circledR}$ Nicotine Free TPM (denoted QTPM) contained $0.056 \mathrm{mg} / \mathrm{mL}$ of nicotine $(0.271$ $\mathrm{mM}$ ). Quantification of the nicotine concentration in each TPM extract enabled direct comparison of TPM effects with a nicotine standard, prepared at the same nicotine concentration (Ambrose et al., 2007).

\subsection{2 - Cell Treatments and Exposures}

Batches of $650 \mathrm{~mL}$ or $250 \mathrm{~mL}$ culture flasks of SH-SY5Y and U-118 MG cells were grown to near confluence in the presence of added volumes of ethanol, purified nicotine, and TPM, from both Holiday ${ }^{\circledR}$ and Quest ${ }^{\circledR}$ brand cigarettes. 
Cell flasks were treated with appropriate volumes of nicotine and TPM to give a final concentration of $0.2 \mu \mathrm{M}$ nicotine in the medium. As the QTPM and ethanol could not be titrated to a known nicotine concentration, volumes of QTPM and ethanol equal to the volumes of nicotine and TPM used were added to these treatment flasks. The volume of ethanol added to each flask was kept constant to control for any cellular effects induced by ethanol. Flasks of untreated SH-SY5Y and U-118 MG cells were prepared as controls as well, to determine the effect of ethanol exposure on the cells. Cells were exposed to concentrations of these extracts for 1,3 , or 5 days, as described in the individual experimental chapters of this thesis. The cells were then harvested and either lysed for enzyme activity measurements and Western blotting analysis, or treated with TRIzol ${ }^{\circledR}$ reagent for PCR analysis.

\section{3-SAMPLE PREPARATION}

Many of the experiments described in this thesis aimed to compare the protein activity and gene expression profile of treated cells with respect to MAO enzymes or MOR. In order to maintain the relationship between protein and gene expression between samples and reduce the sample variation, the samples used for these experiments were prepared from the same flasks of treated cells. That is, flasks of cells were grown and exposed to tobacco compounds and upon harvesting, cells were divided so that some were prepared for RNA extraction and the rest lysed for protein analysis. The methods used for these preparations are detailed below.

\subsection{1 - Protein Lysate Preparation}

Flasks of SH-SY5Y and U-118 MG cells previously treated with tobacco compounds were detached from the flask surface with trypsin and centrifuged, as described above. The cell pellet was then resuspended in complete medium (RPMI 1640, 10\% foetal calf serum, $1 \%$ Penstrep) in $15 \mathrm{~mL}$ plastic centrifuge tubes (Greiner Bio-one $\mathrm{GmbH}$. Germany), and cell numbers were assessed by haemocytometer to determine the concentration of cells in suspension. 
The cell suspension was centrifuged at $300 \times \mathrm{g}$ as before for $5 \mathrm{~min}$ and the medium supernatant removed by aspiration. The cell pellet was then resuspended in $50-200 \mu \mathrm{L}$ of lysis buffer ( $1 \mathrm{mM} \mathrm{MgCl2}, 2 \mathrm{mM}$ EGTA, 1\% Nonidet P40, $50 \mathrm{mM}$ Tris) and homogenized by tituration with a pipette. The cell lysate solution was removed to a clean, sterile $1.5 \mathrm{~mL}$ microcentrifuge tube and centrifuged at $13,000 \mathrm{~g}$ for $10 \mathrm{~min}$ at $4^{\circ} \mathrm{C}$ in an Eppendorf benchtop centrifuge to pellet any insoluble components. Lysates were immediately frozen and stored at $-80^{\circ} \mathrm{C}$ until assayed for MAO-A and MAO-B enzyme activity or used for Western blotting.

\section{$\underline{2.3 .2-\text { TriZol }^{\circledR} \text { Total RNA Extraction }}$}

After assessing the cell concentration by haemocytometer, appropriate aliquots of the cell suspension were transferred to sterile $1.5 \mathrm{~mL}$ microcentrifuge tubes to give approximately $1 \mathrm{x}$ $10^{6}$ cells per tube. The microcentrifuge tubes were then centrifuged at room temperature (RT) for $5 \mathrm{~min}$ at $300 \mathrm{~g}$ in a Sigma 1-14 benchtop centrifuge. The medium supernatant was removed by aspiration, and TRIzol ${ }^{\circledR}$ reagent (Invitrogen Ltd., CA, USA) was added to the cell pellet. Volumes of $1 \mathrm{~mL}$ of TRIzol ${ }^{\circledR}$ per $1 \times 10^{6}$ cells were used, and the cell pellet was lysed and dispersed with repeated pipetting to give a homogeneous solution. Cell lysates prepared with TRIzol ${ }^{\oplus}$ were then frozen at $-80^{\circ} \mathrm{C}$ for up to 3 months, until required for RNA extraction.

\subsection{3 - Protein Concentration Assay}

Cell lysates prepared with lysis buffer and stored at $-80^{\circ} \mathrm{C}$ were thawed and assayed for total protein concentration using a BCA protein assay kit (Pierce. Rockford, IL, USA.). The kit instructions were followed exactly for the microwell plate procedure. Briefly, a small aliquot of lysate was diluted 1:10 with sodium phosphate buffer (50 mM sodium phosphate, $150 \mathrm{mM}$ sodium chloride, $\mathrm{pH} 7.4$ ), and $25 \mu \mathrm{L}$ samples of the diluted lysate were assayed in triplicate. Bovine serum albumin (BSA) protein standards were prepared at concentrations of 0 - 2000 $\mu \mathrm{g} / \mathrm{mL}$. The protein standards and samples $(25 \mu \mathrm{L})$ were incubated with $200 \mu \mathrm{L}$ of working $\mathrm{BCA}$ reagent each at $37^{\circ} \mathrm{C}$ for $30-60 \mathrm{~min}$ and allowed to cool to room temperature before the absorbance was recorded at $562 \mathrm{~nm}$ with a Versamax microplate reader (Molecular Devices, Sunnyvale, CA, USA). The absorbance of the protein standards was used to construct a standard curve for estimation of the total protein concentration of the lysate samples. 


\section{4-MONOAMINE OXIDASE ACTIVITY ASSAYS}

Measurement of the monoamine oxidase enzyme activity in cultured human cells comprised a major part of the experimental work for this project. Two different assays were tested and compared for their reliability, sensitivity, and appropriateness for the experiments required. The first of these was the Amplex $\operatorname{Red}^{\circledR}$ monoamine oxidase enzyme assay, obtained commercially from Molecular Probes, Ltd (Eugene, OR, USA). Additionally, a modified method of the monoamine oxidase enzyme assay utilizing the fluorescent MAO substrate kynuramine as reported by Morinan and Garrett (1985) was developed and optimized. The kynuramine assay was used for the bulk of the MAO enzyme experiments reported here, and further adapted and refined as required. These reactions were used to assess the MAO enzyme activity in total cell lysates, and in purified recombinant MAO-A and MAO-B enzymes, obtained commercially from Sigma-Aldrich, Inc (St Louis, MO, USA). The procedures used to conduct the Amplex $\operatorname{Red}^{\circledR}$ and Kynurmaine assays are described below.

\subsection{1 - Amplex ${ }^{\circledast}$ Red Monoamine Oxidase Activity Assay}

The Amplex ${ }^{\circledR}$ Red Monoamine Oxidase Assay kit (Molecular Probes, A12214) was trialed for measuring MAO-A and MAO-B enzyme activity in cultured cells. The manufacturer's instructions were followed exactly. Briefly, cell lysates were prepared as described above (Section 2.3.1), and assayed for total protein concentration using the BCA assay kit. Protein lysate solutions were diluted to a known standard concentration with 1X Reaction Buffer, and $100 \mu \mathrm{L}$ aliquots of diluted lysate were added to the wells of a black 96-well microplate (Greiner BioOne $\mathrm{GmbH}$, Germany). A positive control was prepared by diluting a 3\% hydrogen peroxide solution to $10 \mu \mathrm{M}$ in reaction buffer, and $100 \mu \mathrm{L}$ of this solution was added to a well in the microplate. The Amplex ${ }^{\circledR}$ Red working solution was prepared (400 $\mu \mathrm{M}$ Amplex ${ }^{\circledR}$ Red reagent, $2 \mathrm{U} / \mathrm{mL}$ horseradish peroxidase, $2 \mathrm{mM} p$-tyramine substrate), and the reaction was initiated by addition of $100 \mu \mathrm{L}$ of Amplex ${ }^{\circledR}$ Red working solution to each $100 \mu \mathrm{L}$ sample (a total volume of $200 \mu \mathrm{L}$ per well). Reactions were incubated in the dark at ambient RT for between 30 min and 2 hours.

The Amplex Red ${ }^{\circledR}$ enzyme assay allowed differentiation of MAO-A and MAO-B activity through the inclusion of different enzyme inhibitors in the assay working solution. Depending on 
which MAO enzyme was being measured, the Amplex Red ${ }^{\circledR}$ working solution also contained $0.5 \mu \mathrm{M}$ pargyline for measuring MAO-A activity, or $0.5 \mu \mathrm{M}$ clorgyline when measuring MAO-B activity.

A standard curve was prepared by diluting a resorufin solution to appropriate concentrations, and $200 \mu \mathrm{L}$ of each resorufin standard was added to the micro-well plate prior to measuring fluorescence. Fluorescence was measured in a SpectraMax Gemini fluorescence multiplate reader (Molecular Devices Ltd., CA, USA.) using an excitation wavelength of $560 \mathrm{nM}$ and emission detection at $590 \mathrm{~nm}$. Measurements were taken either at one final time point (endpoint measurement), or once every 2 min over the course of the total reaction incubation time (up to 2 hrs, for kinetic measurements).

\subsection{2 - Kynuramine Monoamine Oxidase Activity Assay}

The kynuramine monoamine oxidase activity assay employed in this study adapted a method previously described by Morinan and Garrett (1985). Modifications to the reported protocol were made to streamline the assay and allow for higher throughput of test samples. This involved adapting the assay for use with smaller volumes and for analysis in a 96-well plate. The different formats of the kynuramine assay used in this study are described below.

\subsubsection{1 - Kynuramine Microcentrifuge Tube Procedure}

Previously prepared and quantified cell lysates or purified MAO-A or MAO-B human recombinant enzyme (Sigma) were thawed at room temperature and maintained on ice until required for testing. Aliquots of $100 \mu \mathrm{L}$ of cell lysate or MAO enzyme were added to $870 \mu \mathrm{L}$ of sodium phosphate buffer (50 mM sodium phosphate, $150 \mathrm{mM}$ sodium chloride, $\mathrm{pH}$ 7.4) in a $1.5 \mathrm{~mL}$ plastic microcentrifuge tube (Eppendorf, GmbH. Hamburg, Germany). The solutions were incubated in a $37^{\circ} \mathrm{C}$ water bath for $5 \mathrm{~min}$ to equilibrate the reaction temperatures. The assay reaction was started with addition of a $30 \mu \mathrm{L}$ aliquot of $3.07 \mathrm{mM}$ kynuramine hydrobromide (Sigma-Aldrich, Inc. St Louis, MO, USA) to each reaction tube (a total reaction volume of $1 \mathrm{~mL}$ ). Addition of the kynuramine substrate was carefully timed, and the reaction tubes were returned to the water bath and incubated at $37^{\circ} \mathrm{C}$ for $15-60 \mathrm{~min}$. The reaction was terminated by addition of $300 \mu \mathrm{L}$ of $0.4 \mathrm{M}$ perchloric acid (Sigma-Aldrich, Inc. St Louis, MO, USA.), to deactivate and precipitate the proteins. The reaction tubes were then 
centrifuged at $13,000 \mathrm{~g}$ for $30 \mathrm{sec}$ at $4^{\circ} \mathrm{C}$ in an Eppendorf refrigerated benchtop centrifuge to pellet any precipitated proteins, as insoluble proteins interfere with the fluorescence measurement.

Between three and five $100 \mu \mathrm{L}$ aliquots of each reaction solution were added to the wells of a sterile black polystyrene 96-well microwell plate (Greiner Bio-one $\mathrm{GmbH}$, Germany). Dilutions of a 4-hydroxyquinoline reference standard were prepared $(0-5000 \mathrm{nM})$ and added in triplicate to the micro-well plate to construct a standard curve. $\mathrm{NaOH}(200 \mu \mathrm{L}$ of $1 \mathrm{M})$ solution was added to each well to cause the 4-hydroxyquinoline product to fluoresce. The micro-well plate was tapped gently several times to ensure adequate mixing of samples before recording fluorescence. Fluorescence was measured immediately using a SpectraMax Gemini spectrophotometer, at $318 \mathrm{~nm}$ excitation and $380 \mathrm{~nm}$ emission wavelengths, respectively. The 4-hydroxyquinoline standard curve was used to quantitate the concentration of product present in each well, and these data were used to calculate the MAO activity of each sample.

\subsubsection{2 - Kynuramine Microplate Procedure}

The micro-tube assay described above was further refined and adapted to be carried out entirely in a 96-well plate format. This allowed higher-throughput of TPM samples to test for MAO inhibition. This method was used as a bio-assay to test TPM samples from different tobacco sources for their effects on the activity of human recombinant MAO-A (Cat. M7316) and MAO-B (Cat. M7441) enzymes, sourced commercially (Sigma-Aldrich, Inc. St Louis, MO, USA).

Previously prepared TPM extracts were diluted to working concentrations with sodium phosphate buffer $(\mathrm{pH} 7.4)$ in sterile plastic microcentrifuge tubes. A constant ethanol concentration of $0.05 \%$ was maintained in these solutions to control for the effects of ethanol on the activity of MAO enzymes. TPM dilutions were added in triplicate to the wells of a black polystyrene 96-well micro-plate (Greiner BioOne, GmbH. Germany) in $100 \mu \mathrm{L}$ aliquots. A 4hydroxyquinoline reference standard was diluted over a range of concentrations to generate a standard curve, and $200 \mu \mathrm{L}$ aliquots of each standard were added to the micro-well plate in triplicate by pipette. A $0.307 \mathrm{mM}$ working solution of kynuramine dihydrobromide was prepared in sodium phosphate buffer (50 mM sodium phosphate, $150 \mathrm{mM}$ sodium chloride, $\mathrm{pH}$ 7.4), and $60 \mu \mathrm{L}$ of this solution was added to each reaction well on the plate. Frozen aliquots of human recombinant MAO-A or MAO-B (Sigma-Aldrich) were reconstituted to 125 $\mu \mathrm{g} / \mathrm{mL}$ by addition of sodium phosphate buffer. The micro-plate reactions were started by 
addition of $40 \mu \mathrm{L}$ of diluted MAO-A or MAO-B enzyme to each reaction well. To allow for auto-fluorescence of the TPM samples, reaction wells containing diluted TPM, kynuramine working solution, and sodium phosphate buffer in place of the MAO enzyme were also prepared. The microwell plate was then covered with aluminium foil to protect it from light, and the plate was incubated at $37^{\circ} \mathrm{C}$ in a Hereaus Christ Hera-Cell incubator for 30 min.

The reaction was terminated by addition of $75 \mu \mathrm{L}$ of $2.5 \mathrm{M} \mathrm{NaOH}$ to each reaction well and reference standard well. This had the dual effect of inactivating the enzyme and causing the 4-hydroxyquinoline product to fluoresce in excess alkali solution. The 96 -well plate was then analysed for fluorescence immediately with an Optima ${ }^{\circledR}$ fluorescent spectrophotometer using excitation and emission wavelengths of $320 \mathrm{~nm}$ and $380 \mathrm{~nm}$, respectively. The 4hydroxyquinoline standards were used to construct a standard curve that was used to quantitate the concentration of product present in each well. Data from reactions that included dilutions of TPM were compared to a control reaction performed with $0.05 \%$ ethanol in place of TPM, to determine the percentage inhibition of MAO enzyme by the TPM extract.

\section{5- WESTERN BLOTTING}

\section{$\underline{2.5 .1 \text { - Polyacrylamide Gel Electrophoresis }}$}

Protein samples of $20-100 \mu \mathrm{g}$ were prepared for electrophoresis by adding appropriate volumes of lysate to $2.5 \mu \mathrm{L} 4 \mathrm{x}$ LDS sample buffer, and $1 \mu \mathrm{L}$ of $10 \mathrm{x}$ reducing agent (both NuPAGE ${ }^{\circledR}$ products from Invitrogen Co., Carlsbad CA, USA). Protein samples were then heated at $70^{\circ} \mathrm{C}$ for $10 \mathrm{~min}$ on a dry heating block and allowed to cool. Prepared samples and a $7 \mu \mathrm{L}$ aliquot of Precision Plus ${ }^{\circledR}$ (BioRad, Hercules CA., USA) molecular weight protein standard were loaded into the wells of a $1.0 \mathrm{~mm} 8-14 \%$ polyacrylamide NuPAGE ${ }^{\circledR}$ protein gel (Invitrogen Co). Gels and loaded proteins were electrophoresed for $1 \mathrm{hr}$ at $200 \mathrm{~V}$ in an Invitrogen XCell Minigel tank filled with electrophoresis tank buffer (NUPAGE ${ }^{\circledR}$ MOPS SDS Running Buffer, Invitrogen Co. CA, USA). A $500 \mu \mathrm{L}$ aliquot of NuPAGE antioxidant was added to the centre chamber of the tank, as per the manufacturer's instructions.

\subsection{2 - Immunoblotting Procedure}


Immobilon FL polyvinylidene difluoride (PVDF) membrane (Millipore Corp, Billerica, MA, USA) was cut to the same dimensions as the Invitrogen mini-gel. The PVDF was then soaked for 5 min in $100 \%$ methanol and rinsed with $\mathrm{ddH}_{2} \mathrm{O}$ before use. The membrane, blotting paper ( 3 $\mathrm{mm}$ thickness), and blotting pads were soaked in Western transfer buffer (refer to Appendix I) for $15 \mathrm{~min}$, and the blotting apparatus (TransBlot ${ }^{\circledR}$ cell, BioRad, Hercules CA, USA) was assembled (see Appendix).

An icepack and a magnetic stirrer bar were added to the $\operatorname{TransBlot}^{\circledR} \operatorname{tank}$, before the tank was filled with Western transfer buffer, to ensure adequate cooling of the apparatus. Proteins present in the mini-gel were transferred to the membrane at $4^{\circ} \mathrm{C}$ for $3 \mathrm{hr}$ at $400 \mathrm{~mA}$. On completion of the transfer, the apparatus was disassembled and the polyacrylamide gel stained in $0.025 \%$ R-250 Coomassie brilliant blue overnight to check for efficient protein transfer. The PVDF membrane was rinsed with $\mathrm{ddH}_{2} \mathrm{O}$, soaked for $5 \mathrm{~min}$ in T-TBS (refer to Appendix I), and blocked overnight in a 5\% non-fat milk powder solution (Anchor Milk, NZ) in T-TBS at $4^{\circ} \mathrm{C}$ on a rocker platform.

After overnight blocking, the membrane was rinsed and washed in T-TBS in triplicate for $5 \mathrm{~min}$ each wash. The membrane was then incubated with $2 \mathrm{~mL}$ of primary antibody solution (see section 2.5.5) diluted in T-TBS. Antibodies for the house-keeping protein $\beta$-tubulin were included in the primary antibody solutions also. The PVDF membrane was exposed to the primary antibody solution for $4 \mathrm{hr}$ on a rocker platform at ambient RT before rinsing and washing three times with T-TBS. The membrane was then incubated with the appropriate secondary antibody conjugated to a $\mathrm{Cy}^{\mathrm{TM}}-5$ fluorophore, as described in section 2.5.5. The secondary antibodies were diluted in T-TBS and applied to the PVDF membrane, before incubating on a rocker platform for one hr at RT.

Immunostained PVDF membranes were then washed 4 times with T-TBS before being visualized on a Fuji-Film FLA-5100 fluorescent scanner. The fluorescence generated by the $\mathrm{Cy}^{\mathrm{TM}}-5$ conjugated secondary antibodies was recorded using the red $635 \mathrm{~nm}$ filter, and $\mathrm{Cy}^{\mathrm{TM}}-3$ stained blots were visualized with the green $532 \mathrm{~nm}$ filter, each producing a 16-bit grayscale TIFF image file. The images obtained from the fluorescent scanner were then used for densitometry analysis with ImageJ software (ImageJ v1.34s, Wayne Rasband, National Institutes of Health, USA), using the integrated pixel density method. The molecular weights of resolved bands were estimated by comparison with the known sizes of the molecular weight markers. 


\section{$\underline{2.5 .3-\text { Antibodies }}$}

Commercially available polyclonal antibodies raised against the MAO-A, MAO-B, and MOR epitopes were sourced, and used to immunostain the PVDF membranes used in the Western blotting experiments. A polyclonal antibody to $\beta$-tubulin, raised in mouse was used to immunostain the blot as a protein loading control.

Secondary antibodies raised against rabbit, goat or mouse IgG epitopes from a donkey host were sourced from Jackson ImmunoResearch Laboratories, Inc (West Grove, PSA, USA). Antibodies raised in donkey were selected to give minimal cross-reaction with the other antibodies used.

Table 3: Primary antibodies used for Western blotting

\begin{tabular}{llll}
\hline Epitope & Host & Supplier & Dilution \\
\hline MAO-A & Rabbit & $\begin{array}{l}\text { Santa Cruz Biotechnology Inc. (Santa Cruz, CA, USA) } \\
\text { (sc-20156) }\end{array}$ & $1: 200$ \\
MAO-B & Goat & Santa Cruz Biotechnology Inc. (sc-18401) & $1: 200$ \\
MOR & Rabbit & Chemicon Intl. (Billerica, MA, USA) (AB5511) & $1: 1000$ \\
B-Tubulin & Mouse & BD Pharmingen (Franklin Lakes, NJ, USA) & $1: 1000$ \\
\hline
\end{tabular}

Table 4: Secondary antibodies used for Western blotting

\begin{tabular}{|c|c|c|c|}
\hline Epitope & Conjugate & Supplier & Dilution \\
\hline Rabbit IgG & $C y^{T M}-5$ & $\begin{array}{l}\text { Jackson ImmunoResearch Laboratories, Inc. } \\
\text { (711-175-152) }\end{array}$ & $1: 500$ \\
\hline Goat IgG & $\mathrm{Cy}^{\mathrm{TM}}-5$ & $\begin{array}{l}\text { Jackson ImmunoResearch Laboratories, Inc. } \\
\text { (705-175-147) }\end{array}$ & $1: 500$ \\
\hline Mouse IgG & $C y^{T M}-5$ & $\begin{array}{l}\text { Jackson ImmunoResearch Laboratories, Inc. } \\
(715-175-150)\end{array}$ & $1: 500$ \\
\hline Mouse IgG & $C y^{T M}-3$ & $\begin{array}{l}\text { Amersham Biosciences, GE Healthcare Ltd. (UK) } \\
\text { (cat PA43009) }\end{array}$ & $1: 500$ \\
\hline
\end{tabular}


The secondary antibodies were conjugated to $\mathrm{Cy}^{\mathrm{TM}}-5$ fluorophores, with the exception of a $\mathrm{Cy}^{\mathrm{TM}}-3$ conjugated anti-mouse secondary antibody which was used to immune-stain the $\beta$ tubulin loading control on the MOR stained blot. Because both the MOR target and $\beta$-tubulin loading control gave immuno-specific bands at approximately $50 \mathrm{kDa}$, staining with different fluorophores and scanning at different wavelengths allowed measurement of both targets without needing to strip the PVDF membrane. The fluorescence generated by the fluorophore-tagged secondary antibodies was visualized with a Fujifilm FLA-5100 Fluorescent image scanner (Fujifilm Global, Tokyo, Japan). This method of Western blot visualization is a more cost and time-efficient alternative to the commonly used luminescent substrate method.

\section{6 - QuANTITATIVE REAL-TIME PCR}

Analysis of mRNA expression of the MAO-A, MAO-B, and MOR genes was performed utilizing the techniques of real-time reverse transcriptase PCR. This allowed relative quantitation of the changes in expression of these genes of interest following exposure to tobacco extracts. These experiments were carried out at the School of Biological Sciences, Victoria University, and the Kenepuru Science Centre, ESR, using a BioRad ${ }^{\circledR}$ iCycler, or a Stratagene MX3005 realtime PCR machine, respectively. The methods used to conduct these experiments are detailed below.

\section{$\underline{2.6 .1-m R N A \text { Extraction }}$}

Flasks of SH-SY5Y and U-118 MG cells were grown to near confluence as described above. The cells were treated with trypsin and detached from the inner surface of the flask as described, collected in a polystyrene centrifuge tube (Greiner Bio-One, GmbH. Germany), and resuspended in fresh RPMI medium. Cell numbers were assessed by haemocytometer. Approximately $1 \times 10^{6}$ cells were transferred to a sterile microcentrifuge tube (Eppendorf, Germany) and centrifuged at $300 \mathrm{~g}$ for 5 mins at $4^{\circ} \mathrm{C}$ in a Sigma 1-14 refrigerated benchtop centrifuge. The supernatant was removed by aspiration. TRIzol ${ }^{\circledR}$ reagent $(1 \mathrm{~mL})$ (Invitrogen, (A, USA) was added to the cell pellet and the suspension was titurated by pipette to resuspend the pellet and lyse the cells. Samples were stored in TRIzol ${ }^{\circledR}$ reagent at $-80^{\circ} \mathrm{C}$ for up to 3 months before extraction. 
To maintain the quality and integrity of extracted RNA, precautions were taken to avoid contamination of the samples with RNases that would degrade the sample. These precautions included using only sterile, certified RNase-free pipette tips and micro-centrifuge tubes in all RNA extraction and handling procedures.

To extract frozen samples stored in TRIzol ${ }^{\circledR}$ reagent, the samples were first thawed at RT for $15 \mathrm{~min}$. A $200 \mu \mathrm{L}$ aliquot of molecular grade chloroform (Sigma-Aldrich, NZ) was added to each tube and the tubes shaken vigorously by hand for $15 \mathrm{sec}$. Samples were incubated at RT for $3 \mathrm{~min}$ and then centrifuged at 10,000 $\mathrm{g}$ for $15 \mathrm{~min}$ at $4^{\circ} \mathrm{C}$ (Sigma 1-14 benchtop centrifuge). The upper aqueous colourless phase containing the RNA was carefully removed by pipette and transferred to sterile, labeled microcentrifuge tubes (Eppendorf, Germany). Absolute ethanol $(500 \mu \mathrm{L})$ was added to each tube to precipitate the RNA, and the solutions were incubated at RT for $10 \mathrm{~min}$.

The samples were then applied to RNA spin cartridges from the Invitrogen Micro-to-Midi RNA Purification $\mathrm{kit}^{\circledast}$ and the kit instructions followed exactly. Briefly, the samples were centrifuged in the spin cartridges for $15 \mathrm{sec}$ at $12,000 \mathrm{~g}$, and the ethanol flow-through was discarded. The spin cartridge was then sequentially washed with the kit wash buffers and centrifuged for $15 \mathrm{sec}$ at $12,000 \mathrm{~g}$, discarding the buffer flow-through between each wash. The cartridge was reassembled with an RNA recovery tube, and total RNA was eluted off the spin cartridge filter into $60 \mu \mathrm{L}$ of DEPC-treated RNase-free water. Total RNA extracts were stored at $-80^{\circ} \mathrm{C}$ until required for reverse transcription.

\subsection{2 - Reverse Transcription}

RNA extracts were tested for concentration and purity with a Nanodrop ND-1000 spectrophotometer, and tested for degradation using an RNA 6000 Nano kit for the Agilent 2100 Bioanalyzer (Agilent Technologies, Inc., USA). The Bioanalyzer is a micro-fluidics based platform which allows rapid gel analysis of RNA samples, giving values for calculated RNA concentration, and RNA Integrity Number (RIN). The RIN is a measure of the quality and integrity of the RNA sample scored out of 10.0, and lower RIN scores indicate partial or significant RNA degradation. For the experiments conducted for this thesis, only RNA samples with an RIN score greater than 9.0, an OD260/OD280 ratio greater than 1.8, and a concentration greater than $80 \mathrm{ng} / \mu \mathrm{L}$ were used for qRT-PCR experiments. This ensured that the RNA used was of very high quality and purity. 
An iScript ${ }^{\circledast}$ cDNA Synthesis kit (BioRad Ltd. Berkely, CA, USA) was used to synthesize firststrand cDNA from the purified RNA samples. The iScript CDNA synthesis kit allows priming of total RNA using oligo-dTs. This method was chosen to amplify the RNA for this study instead of using gene specific primers, since the mRNA for several genes was to be amplified from each RNA sample. Oligo-dT priming allows the amplification of several RNA targets from the same sample. The iScript kit also provides Reverse Transcriptase pre-blended with RNase inhibitors to eliminate RNA degradation by RNases. When preparing the RT reactions the manufacturer's instructions were followed exactly. Briefly, $500 \mathrm{ng}$ of purified total RNA was added to $4 \mu \mathrm{L}$ of $5 \mathrm{x}$ iScript reaction mix and $1 \mu \mathrm{L}$ of iScript reverse transcriptase. Sufficient nuclease-free water was added to bring the reaction mixture to a $20 \mu \mathrm{L}$ final volume. The reaction tubes were incubated for $5 \mathrm{~min}$ on a $25^{\circ} \mathrm{C}$ dry heat block, and then for $30 \mathrm{~min}$ at $42^{\circ} \mathrm{C}$. Reverse transcription reactions were terminated by incubating the tubes at $85^{\circ} \mathrm{C}$ for $5 \mathrm{~min}$, then chilling on ice. Synthesized CDNA was stored at $-20^{\circ} \mathrm{C}$ for up to 3 months before being used for PCR analysis.

\subsection{3 - Quantitative Real-Time PCR}

Quantitative PCR was conducted on either the BioRad iCycler ${ }^{\circledR}$ instrument, or the Stratagene MX3005 instrument using the fluorescent, double-stranded DNA-specific dye SYBR Green. This allows real-time monitoring of the increase in PCR product after every amplification cycle, based on the increase in SYBR Green fluorescence.

Previously prepared cDNA template $(1 \mu \mathrm{L})$ was added to a Platinum SYBR Green qPCR SuperMix-UDG (Invitrogen, 11733-046). For the iCycler ${ }^{\circledast}$ instrument, this supermix comprised $50 \%$ SYBR Green, $20 \mathrm{nM}$ fluorescein, and $200 \mathrm{nM}$ primers to give a total reaction volume of 25 $\mu \mathrm{L}$. Addition of fluorescein is necessary for the PCR detection system used by the iCycler instrument to measure background fluorescence (well factors). Background fluorescence is measured in individual wells at the initiation of each PCR experiment and used to correct for non-uniformity in fluorescence. For the Stratagene instrument, the SYBR-Green supermix was comprised of $50 \%$ SYBR Green, $200 \mathrm{nM}$ primers, and the fluorescein was replaced with $50 \mathrm{nM}$ ROX dye, for measurement of well factors, as per the manufacturer's instructions.

Reaction mixtures were added to thin-walled polypropylene PCR strip tubes, and the strips mounted in the real-time PCR instrument. Samples were amplified using a pre-set protocol 
with the following cycling conditions. The cycling protocol began with an initial step of $50^{\circ} \mathrm{C}$ for $2 \mathrm{~min}$ prior to amplification to activate uracil-N-glycosylase (included in the SYBR Green SuperMix) which degrades any contaminating RNA template. The cDNA was denatured for 2 min at $95^{\circ} \mathrm{C}$, and amplification was carried out over 35 cycles of $95^{\circ} \mathrm{C}$ for $15 \mathrm{sec}$ (denaturation); $60^{\circ} \mathrm{C}$ for $30 \mathrm{sec}$ (annealing); $72^{\circ} \mathrm{C}$ for $30 \mathrm{sec}$ (elongation). The cycling protocol was completed with a melt curve sequence which analysed the temperature at which the PCR products dissociated. This step was performed as a check for PCR product specificity. PCR products were then held at $4^{\circ} \mathrm{C}$ until removed from the PCR instrument. A primer annealing temperature of $60^{\circ} \mathrm{C}$ was used for all primer sets.

\subsection{4 - PCR Primer Design and Validation}

Primers specific for the amplification of the genes of interest to this study were designed and synthesized or supplied as described below.

DNA-directed RNA polymerase II subunit F (POLR2F) was selected as a reference gene based on the findings of a study to identify reference genes in SH-SY5Y cells that would be suitable for qRT-PCR (Hoerndli et al., 2004). The primer pair sequence for POLR2F was sourced from Hoerndli et al. (2004), and the primer specificity was analysed and validated as for the other primers, as described above. These primers amplify a $65 \mathrm{bp}$ segment of the human POLR2F gene, which lies within exon 5. The amplicon runs from position 383 to 438 of the mRNA transcript. Stable expression of POLR2F was validated by comparison to two other housekeeper genes, Glyceraldehyde-3-Phosphate dehydrogenase (GAPDH) and Myosin phosphatase-Rho interacting protein (M-RIP), as described in section 6.3.1.2 of this thesis. GAPDH and M-RIP primer sequences were also sourced from Hoerndli et al. (2004).

Primer pairs for human MAO-A and MAO-B had been previously designed and validated by Michael Green and Dr Rod Lea at ESR Kenepuru Science Centre. The MAO-A primers amplify a $457 \mathrm{bp}$ section of the MAO-A gene, which spans an area of the gene running from exon 8 to exon 13. This section runs from position 1002 to position 1458 of the mRNA transcript. The MAO-B primers amplify a 104 bp segment of the human MAO-B mRNA, from position 1288 to position 1391. This PCR product spans exons 11 and 12 of the human MAO-B gene. The specificity of these primers to the intended genes was confirmed by BLAST search for the predicted amplicon. Primer specificity was further checked by analysis of the PCR products by 
agarose gel electrophoresis, and PCR product dissociation curve analysis with every $\mathrm{qPCR}$ assay.

PCR primers for the specific amplification of human MOR and human eukaryotic initiation factor $4 a$ isoform 2 (elF4A2) were designed using Invitrogen OligoPerfect ${ }^{\circledast}$ software. Optimum conditions were selected for when designing PCR primers, including an optimum primer size of $20 \mathrm{bp}$, melting temperature of $60^{\circ} \mathrm{C}$, and GC content of $50 \%$. Wherever possible, primers were designed to span at least one intron of the gene of interest. This helps ensure that any PCR product is the result of specific amplification of the first-strand CDNA derived from extracted mRNA, and not amplification of gDNA.

The hMOR primers amplify a $230 \mathrm{bp}$ section of human MOR-1 that spans exons 2 and 3 . The amplicon runs from positions 826 to 1052 of the mRNA transcript of this gene. The elF4A2 primers amplify a 220 bp section of the human elG4A2 gene, from position 53 to position 272 . This amplicon spans exons $1,2,3$, and 4 of the gene. Vector $\mathrm{NTI}^{\circledR}$ software was used to confirm the predicted amplicon generated by these primers, and to perform a BLAST search to determine specificity for the gene of interest. Primer specificity was also checked by agarose gel analysis of the PCR products to determine that they conformed to the predicted amplicon size.

Quantitative real-time melt curve analysis was performed as part of every real-time PCR experiment. As each double-stranded DNA product has its own specific melting temperature based on the GC content of the product, melt curve analysis checks the identity of a product and determines the presence of any non-specific products like primer dimers.

Table 5: Primer pairs used for qRT-PCR

\begin{tabular}{|c|c|c|c|}
\hline \multirow[t]{2}{*}{ Primer Target } & \multicolumn{2}{|c|}{ Primer Sequence $5^{\prime}-3^{\prime}$} & \multirow{2}{*}{$\begin{array}{l}\text { Product size } \\
\text { (bp) }\end{array}$} \\
\hline & Forward & Reverse & \\
\hline MAO-A & CTC CGA CCT TGA CTG CCA AG & GGT TGA CGA ATC ACC CTT CC & 457 \\
\hline MAO-B & ACA CAC TGG AGC GGC TAC AT & TGC CAG ATT TCA TCC TCT GGA & 104 \\
\hline hMOR & TCT TCA GCC ATT GGT CTT CC & TCT TTG GAG CCA GAG AGC AT & 230 \\
\hline elF4A2 & CCG CGG ATT ATA ACA GAG AA & TGA GCT TGA GCA ATC ACA TC & 220 \\
\hline POLR2F & CCC GAA AGC TCC CCA TCA T & CAC CCC CCA GTC TTC ATA GC & 65 \\
\hline
\end{tabular}




\subsubsection{1 - Agarose Gel Electrophoresis}

After amplification by conventional or real-time PCR, cDNA samples were analysed by agarose gel electrophoresis to determine the size of the amplicon. Sufficient $5 x$ DNA loading dye was added to the DNA samples to give a final concentration of $1 \mathrm{x}$ loading dye before the samples were pipetted into the wells of a $1 \%$ agarose gel containing $1 \mu \mathrm{g} / \mathrm{mL}$ of ethidium bromide. A 7 $\mu \mathrm{L}$ aliquot of $1 \mathrm{~kb}+$ DNA ladder (100 - $1000 \mathrm{bp}$, Invitrogen) was loaded onto the agarose gel also, to serve as a molecular weight standard. Electrophoresis was performed at $100 \mathrm{~V}$ for approximately $45 \mathrm{~min}$, or until the dye front had migrated sufficiently through the gel.

The gels were checked for DNA bands by visualization with a UV-transilluminator (UVP, Inc. Upland, CA), before capturing a digital photograph of the gel with a Kodak ${ }^{\circledR}$ Gel Logic 100 digital imaging system.

\section{$\underline{2.7-\text { STATISTICAL ANALYSIS }}$}

Statistical analyses and tests on all data were performed using Graphpad Prism v5.0 (Graphpad software, Inc. San Diego, CA). Tests performed included student's t-tests, and oneand two-way Analysis of Variance (ANOVA), with relevant post-hoc tests including the Bonferroni multiple comparison post-test and Dunnett's post-test. Dose-response relationships and enzyme reactions were analysed by non-linear regression analysis, as noted. Graphpad Prism v5.0 and Microsoft Excel 2007 were used for graphing and data manipulation. The details of the tests performed will be described with the results of the individual experimental chapters of this thesis. 


\section{Chapter Three: \\ Development of a fluorimetric MAO enzyme activity assay}

The aim of a major part of this study was to assess the effects of tobacco compounds on the enzymatic activities of MAO-A and MAO-B under a number of different conditions. To obtain meaningful data on the effects of tobacco on MAO activity, a fast, sensitive, and robust quantitative enzymatic assay was required. Because this study intended to test a large number of samples from various extracts and tissue, the preferred assay method needed to be suitable for rapid, high-throughput processing of these samples.

Historically, a number of different methods have been developed and employed to detect and quantify the activity of MAO enzymes in vitro and in vivo. Many of these studies have utilized radio-labelled MAO substrates to measure enzyme activity. For example, O'Carroll et al. (1983) used $\left[{ }^{14} \mathrm{C}\right]$-dopamine as a substrate for MAO in human brain tissue homogenates, measuring the accumulation of the deaminated radio-labelled products. The study also employed the inhibitors I-deprenyl and clorgyline to distinguish between the activity of MAOA and MAO-B, respectively. Yu \& Boulton (1987) assessed the inhibition of MAO in rat lung homogenates following tobacco smoke exposure with a similar radio-chemical assay that used different substrates to differentiate MAO-A from MAO-B activity. Radio-labelled 5hydroxytryptamine and $\beta$-phenylethylamine were the substrates used for MAO-A and MAO-B, respectively. Similarly, Hauptmann \& Shih (2001) measured inhibition of MAO-A and MAO-B in rat mitochondrial preparations using $\left[{ }^{14} \mathrm{C}\right]$-serotonin and $\left[{ }^{14} \mathrm{C}\right]$-phenylethylamine, and Rose et al. (2001) measured MAO-B activity in human blood platelets using $\left[{ }^{14} \mathrm{C}\right]$-benzylamine.

While radio-chemical assays are sensitive and can be designed to use the enzyme's native substrates, many laboratories prefer to avoid the added inconvenience of working with radioactive isotopes. Other quantitative MAO enzyme assays exploit various characteristics of the deamination reaction catalysed by MAO. As discussed earlier (section 1.3.1), MAO requires molecular oxygen to oxidize monoamine substrates to an aldehyde intermediate, producing hydrogen peroxide as a by-product. Several studies have directly assessed MAO 
activity through measuring the consumption of oxygen by the enzyme using a polarographic oxygen electrode (Krueger \& Singer, 1993; Sweetman \& Weetman, 1969). This method is reportedly very accurate and reproducible, but is unsuitable for rapid high-throughput testing of large numbers of samples.

\subsection{1 - Amplex Red Assay}

The Amplex ${ }^{\circledast}$ Red assay described by Zhou \& Panchuk-Voloshina (1997) measures accumulation of the hydrogen peroxide by-product produced during the MAO deamination reaction to quantitate enzyme activity. Amplex ${ }^{\circledR} \operatorname{Red}(\mathrm{N}$-acetyl-3,7-dihydroxyphenoxazine) is a highly sensitive fluorescent probe which, in the presence of horseradish peroxidase, is converted by hydrogen peroxide to the stable fluorescent product resorufin. The assay has been developed as a commercially available kit for the fluorimetric measurement of MAO enzyme activity (Molecular Probes Ltd., Invitrogen). The Amplex ${ }^{\circledR}$ Red assay can be performed in a 96-well microplate format which is suitable for high-throughput testing, and the kit design allows measurement of enzyme activity at the reaction end-point or continuously for kinetic measurements. The fluorescent product detected in this assay resorufin - has a fluorescence maximum of approximately $585 \mathrm{~nm}$, giving this assay the advantage of being largely unaffected by autofluorescence in most biological samples. The kit has been designed to selectively distinguish between the activity of MAO-A and MAO-B through the use of the selective MAO-A and MAO-B inhibitors clorgyline and pargyline, respectively.

The Amplex Red 96-well microplate kit has been used to test MAO enzyme activity in a number of different enzyme preparations with good sensitivity. Zhou \& Panchuk-Voloshina (1997) reported the measurement of MAO-B activity from $0.0125 \mathrm{U} / \mathrm{mL}$ of purified MAO-B enzyme, and the detection of MAO-A and MAO-B enzyme activity from bovine brain homogenates containing $200 \mu \mathrm{g}$ of total protein per reaction. Snell et al. (2002) successfully used the Amplex ${ }^{\circledR}$ Red kit to examine MAO-B activity in human blood platelets using $15 \mu \mathrm{g}$ of total protein per well. Another study by Guang \& Du (2006) distinguished MAO-A and MAO-B enzyme activity in rat brain mitochondrial homogenates with a final protein concentration of $0.2 \mathrm{mg} / \mathrm{mL}$, using serotonin as a substrate for MAO-A and benzylamine as a substrate for MAO-B. 


\subsection{2 - Kynuramine Assay}

Although the Amplex ${ }^{\circledR}$ Red kit is reportedly very sensitive, it measures MAO enzyme activity indirectly via a coupled reaction, which increases the complexity of the assay and can complicate data interpretation. The kynuramine assay is another commonly used fluorimetric assay that measures the activity of MAO directly, rather than through a coupled reaction. Kynuramine is a substrate for both MAO-A $\left(\mathrm{K}_{\mathrm{m}}=42 \mu \mathrm{M}\right)$ and MAO-B $\left(\mathrm{K}_{\mathrm{m}}=26 \mu \mathrm{M}\right)$ (Yan et al., 2004), which catalyze the deamination of kynuramine to the product 4-hydroxyquinoline (4$\mathrm{HQ}$ ), also known as quinolinol. 4-HQ fluoresces in the presence of strong alkali conditions at an excitation wavelength of $318 \mathrm{~nm}$ and an emission wavelength of $380 \mathrm{~nm}$ (Massey \& Churchich, 1977).

The kynuramine assay was first described by Kraml (1965), and revised by Morinan \& Garrett (1985), who significantly increased the sensitivity of the assay. Morinan \& Garrett (1985) measured MAO activity in rat brain homogenates and tested the effects of a number of selective and non-selective MAO inhibitors. They detected MAO activity in homogenates using $500 \mu \mathrm{g}$ total protein per reaction, and reported the lower limit of detection for pure 4$\mathrm{HQ}$ product as $500 \mathrm{fM}$. A number of other studies have used variations of the basic kynuramine assay such as Mahmood et al. (1994) who assayed rat brain homogenates for MAO-B activity following exposure to selegiline, a selective MAO-B inhibitor. In addition, Mendez-Alvarez, et al. (1997b) used kynuramine to measure the inhibition of MAO-A and MAO-B in rat brain mitochondria in response to treatment with a number of compounds isolated from tobacco smoke, and Rommelspacher, et al. (2002) measured MAO-B activity in the blood platelets of human smokers.

More recently, the kynuramine assay has been developed further to provide more sophisticated detection of the 4-HQ fluorescent product. Yan et al. (2004) incubated human recombinant MAO-A and MAO-B enzymes with kynuramine in 96-well microplates before analyzing the reactions for 4-HQ concentration by liquid chromatography with tandem mass spectrometry (LC/MS/MS). They state their method provides a highly sensitive and specific assay that can be used for high through-put screening of MAO inhibitors. Herraiz \& Chaparro (2005) also used recombinant human MAO-A and MAO-B with kynuramine and detected the 4-HQ reaction product using reverse phase high performance liquid chromatography (RPHPLC) and fluorescence detection. They successfully used their modified technique to determine whether two compounds isolated from tobacco smoke extract inhibited MAO enzymes. 


\subsection{3 - Other MAO Assays}

Other more sophisticated methods have also been recently developed to assay for MAO enzyme activity. The MAO Glo ${ }^{\circledR}$ assay (Promega Corporation, WI) uses a derivative of beetle luciferin as a substrate for MAO, and the reaction product emits light with addition of luciferase. Like the Amplex $\operatorname{Red}^{\circledR}$ kit, this assay is performed in a 96-well microplate, making it suitable for high-throughput testing, and it claims greater sensitivity and stability of the luminescent signal. Chen et al. (2005) describe the development of a stable, irreversible fluorogenic redox switch specifically designed to be a substrate for MAO-A and MAO-B, which they validated by detecting MAO-A and MAO-B in human placental mitochondria and bovine liver mitochondria preparations, respectively. However, this assay has not yet been used to collect data on biological samples.

Zitova et al. (2009) developed an assay to measure oxygen consumption by the MAO reaction using the Roche LightCycler ${ }^{\circledR}$ detection platform, which was originally developed for quantitative PCR. Their respirometric assay used low volume reactions $(20 \mu \mathrm{L})$ of human recombinant MAO-A and MAO-B with dopamine or benzylamine as substrate, and the reaction was carried out in glass capillary tubes on the LightCycler ${ }^{\circledR}$ system. MitoXpress, a macromolecular oxygen probe, was used to detect changes in molecular oxygen in the reactions. This innovative approach is reportedly more sensitive than other fluorescencebased oxygen consumption assays and can accurately monitor the activity of other oxygenase enzymes, such as cytochrome P450 (CYP) and cyclo-oxygenase (COX).

In summary, many different methods have been described for quantitative assessment of the activity of MAO enzymes. To accurately measure changes in MAO enzyme activity following exposure to tobacco compounds, the present study required an assay that was robust, sensitive, and selective for MAO activity. The selected assay needed to be rapid and relatively inexpensive to make it suitable for high-throughput screening of numerous samples derived from several different tissues.

\section{$\underline{3.1 .4-\text { Objectives }}$}

The aim of the experiments in this chapter was to assess the effectiveness, reliability and sensitivity of a high-throughput assay for measuring changes in the activity of MAO-A and MAO-B enzymes in a number of different biological samples. The commercially available Amplex ${ }^{\circledR}$ Red monoamine oxidase assay was trialed initially, but gave mixed results (see 
Results, section 3.3.1). The kynuramine assay was then developed by making appropriate modifications to published protocols and by thorough testing of the reaction parameters. A number of tests were used to validate the accuracy and reliability of the kynuramine assay, and the results were briefly compared to those achieved with the Amplex Red assay. The kynuramine assay was then used to assess MAO inhibition in a pilot study of human blood platelet samples to demonstrate the use of the assay as a proof of the concept.

The assessment of each of these assays led to the optimization of the methods that were subsequently used extensively throughout this study. The more consistent and repeatable kynuramine assay system was used to measure the activity of purified recombinant MAO enzymes and enzymes in lysates of cultured cells exposed to tobacco products.

\section{2 - MATERIALS AND METHODS}

\subsection{1 - Sample Preparation}

The MAO enzyme activity assays used in these experiments were tested and optimized using several different sources of MAO-A and MAO-B enzyme. These included cell lysates prepared from cultured SH-SY5Y neuroblastoma cells, cultured U-118 MG glioma cells, human peripheral blood platelets, and purified recombinant MAO-A and MAO-B enzymes. These samples were prepared as described below.

\subsubsection{1 - Cultured Cell Lysates}

Cell lysates were prepared from cultured SH-SY5Y neuroblastoma and U-118 MG glioma cell lines as described in section 2.3.1. Briefly, cells were pelleted by centrifugation and resuspended in lysis buffer ( $1 \mathrm{mM} \mathrm{MgCl} 2,2 \mathrm{mM}$ EGTA, 1\% Nonidet P40, $50 \mathrm{mM}$ Tris), before storage at $-80^{\circ} \mathrm{C}$. When required for testing, lysates were thawed at RT for 10 mins and assayed for total protein content using a BCA assay (see section 2.3.3). Protein samples were then diluted to appropriate working concentrations with sodium phosphate buffer ( $50 \mathrm{mM}$ sodium phosphate, $150 \mathrm{mM}$ sodium chloride, $\mathrm{pH} 7.0$ ) before testing. 


\subsubsection{2 - Human Blood Platelets}

Venous blood samples were drawn from human subjects by a trained phlebotomist into $5 \mathrm{~mL}$ vacutainer tubes. The samples were immediately centrifuged at $600 \mathrm{xg}$ at $4^{\circ} \mathrm{C}$ for 4 mins in a Sigma 3-16 benchtop centrifuge to separate red blood cells from the platelet-rich plasma. The plasma layer was transferred by pipette to a clean, sterile microcentrifuge tube and centrifuged at $10,000 \times \mathrm{g}$ at $4^{\circ} \mathrm{C}$ for 5 mins to pellet the platelets. The plasma supernatant was removed by aspiration, and the platelet pellet was stored at $-80^{\circ} \mathrm{C}$ until required. The platelet pellet was then resuspended in sodium phosphate buffer and assayed for total protein content by BCA assay. Platelet samples were then diluted with sodium phosphate buffer to appropriate protein concentrations before measurement of MAO activity.

\subsubsection{3 - Purified Recombinant MAO-A and MAO-B Enzymes}

Purified human recombinant MAO-A (Cat. M7316) and MAO-B (Cat. M7441) enzymes (5 $\mathrm{mg} / \mathrm{mL}$ ) were purchased from Sigma-Aldrich, Inc. for use in this study. Vials of frozen enzyme were shipped on dry ice and immediately transferred to a $-80^{\circ} \mathrm{C}$ freezer on arrival. The vials were then thawed rapidly by hand and maintained on ice while the protein solutions were pipetted in $10 \mu \mathrm{L}$ aliquots into sterile microcentrifuge tubes. These aliquots were then returned to the freezer and stored at $-80^{\circ} \mathrm{C}$ until required. Before use, aliquots were thawed rapidly and diluted to working concentrations with sodium phosphate buffer.

\subsection{2 - Invitrogen Amplex ${ }^{\circledR}$ Red Assay}

The Amplex ${ }^{\circledast}$ Red Monoamine Oxidase Assay kit (Molecular Probes, A12214) was purchased from Invitrogen. The manufacturer's instructions were followed exactly and are described in detail in section 2.4.1 of this thesis. Briefly, prepared lysates were diluted with $1 \mathrm{x}$ reaction buffer, and $100 \mu \mathrm{L}$ aliquots of diluted lysate were added in triplicate to the wells of a black 96well microplate (Greiner BioOne). Wells containing $100 \mu \mathrm{L}$ of $10 \mu \mathrm{M}$ hydrogen peroxide or $1 \mathrm{x}$ reaction buffer were also included as positive and negative controls, respectively. The Amplex ${ }^{\circledast}$ Red working solution was prepared (400 $\mu \mathrm{M}$ Amplex ${ }^{\circledast}$ Red reagent, $2 \mathrm{U} / \mathrm{mL}$ horseradish peroxidase, $2 \mathrm{mM} p$-tyramine substrate, $\pm 1 \mu \mathrm{M}$ inhibitor), and the reaction was 
initiated by addition of $100 \mu \mathrm{L}$ of Amplex $^{\oplus}$ Red working solution to each $100 \mu \mathrm{L}$ sample. A negative control containing aliquots of cell lysate, reaction buffer, and Amplex Red working solution without the $p$-tyramine substrate was included for all experiments. Reactions (200 $\mu \mathrm{L}$ ) were incubated at RT in the dark for between 30 mins and 2 hours.

The Amplex ${ }^{\circledR}$ Red assay allows the measurement of both MAO-A and MAO-B enzyme activity through the use of different inhibitors. When measuring MAO-A activity, $0.5 \mu \mathrm{M}$ pargyline was included to inhibit MAO-B, and when measuring MAO-B activity the reaction buffer contained $0.5 \mu \mathrm{M}$ clorgyline to inhibit MAO-A. When measuring total MAO enzyme activity, no inhibitor was included in the reactions.

Fluorescence was measured with a SpectraMax Gemini fluorescence spectrophotometer (Molecular Instruments Ltd), using a kinetic measurement protocol. Fluorescence was measured once every 2 mins over the duration of the incubation period using an excitation wavelength of $560 \mathrm{~nm}$ and emission detection at $590 \mathrm{~nm}$.

The resorufin reference standard provided with the kit reagents was diluted over the range from $0.5 \mu \mathrm{M}$ to $20 \mu \mathrm{M}$, and aliquots of these standards were included in each microwell plate. The fluorescence of these standards was measured once at the start of the reaction, and was then used to construct a standard curve to quantitate the concentration of resorufin product present in each well, and thus the MAO activity of each sample.

\subsubsection{1 - Using Amplex Red to Assess MAO Activity in Differentiated SH-SY5Y Cells}

Amplex Red was used in early experiments with SH-SY5Y human neuroblastoma cells to measure changes in MAO enzyme activity following differentiation with retinoic acid. SH-SY5Y cells were sub-cultured into $250 \mathrm{~mL}$ tissue culture flasks at known densities according to the methods described in section 2.1. The cells were incubated in growth media overnight and allowed to adhere to the substrate before addition of retinoic acid (RA) to a final concentration of $10 \mu \mathrm{M}$. The growing cells were then incubated with RA for $1,5,7$, or 10 days, refreshing the medium and RA solution every 4 days. The cells were then harvested and prepared as previously described (section 3.2.1) before each sample was tested in triplicate with the Amplex Red assay for MAO-A activity, MAO-B activity, and total MAO activity. 


\subsection{3 - Kynuramine Assay}

The modified kynuramine assay used in these experiments is described in detail in section 2.4.2 of this thesis. The experimental protocol for this assay is described briefly below.

Samples of known protein concentration were dispensed in $100 \mu \mathrm{L}$ aliquots to $1.5 \mathrm{~mL}$ microcentrifuge tubes containing $870 \mu \mathrm{L}$ of sodium phosphate buffer (50 mM sodium phosphate, $150 \mathrm{mM}$ sodium chloride, $\mathrm{pH}$ 7.4). These sample solutions were incubated in a $37^{\circ} \mathrm{C}$ water bath for 5 mins to equilibrate to the reaction temperature. The assay reaction was initiated by addition of a $30 \mu \mathrm{L}$ aliquot of kynuramine hydrobromide (Sigma-Aldrich, Inc) to each reaction tube, giving a total reaction volume of $1 \mathrm{~mL}$. Reactions nominally contained $3.07 \mathrm{mM}$ kynuramine substrate unless otherwise stated. The reaction tubes were then returned to the water bath and incubated at $37^{\circ} \mathrm{C}$ for $15-60 \mathrm{~min}$. The enzyme reaction was terminated by addition of $300 \mu \mathrm{L}$ of $0.4 \mathrm{M}$ perchloric acid (Sigma-Aldrich, Inc), and the reaction tubes were then centrifuged at $13,000 \times \mathrm{g}$ for $30 \mathrm{sec}$ at $4^{\circ} \mathrm{C}$ in an Eppendorf refrigerated benchtop centrifuge.

Following centrifugation, $100 \mu \mathrm{L}$ aliquots of each supernatant were added in triplicate to the wells of a sterile black polystyrene 96-well microwell plate (Greiner Bio-one). Dilutions of a 4$\mathrm{HQ}$ reference standard were prepared $(0-5000 \mathrm{nM})$ and added in triplicate to the micro-well plate to construct a standard curve. $\mathrm{NaOH}$ ( $200 \mu \mathrm{L}$ of $1 \mathrm{M}$ solution) was added to each well, and fluorescence was measured immediately using a SpectraMax Gemini spectrophotometer at $318 \mathrm{~nm}$ excitation and $380 \mathrm{~nm}$ emission wavelengths. The 4-HQ standard curve was used to quantitate the concentration of product present in each well.

\subsubsection{1 - Assessing Assay Variation}

Variation can arise and influence an enzymatic assay from a number of sources. These include biological variation in the activity of the enzyme in the source sample (between-sample variation) and the inherent variability of the assay procedure due to inaccuracies in pipetting, timing, and incubation temperature.

To assess the between-sample variability of the kynuramine assay, total MAO-A activity was measured in untreated $\mathrm{SH}-\mathrm{SH} 5 \mathrm{Y}$ cell lysates assayed independently of each other, and the results compiled to examine the spread of the data. 
To examine variability of the assay itself, three independently prepared untreated SH-SY5Y lysates were prepared by harvesting and lysing the cells as described above (section 3.2.1.1), and the lysates assayed for total protein concentration. Each lysate was then divided evenly between two microcentrifuge tubes and the samples were then frozen at $-80^{\circ} \mathrm{C}$ and stored for up to a month before testing. One of each prepared lysate was removed from the freezer and tested for total MAO activity in triplicate according to the standard protocol described. On the following day, the remaining aliquots of each lysate were tested for total MAO activity in triplicate, and the results from each assay were compared.

\subsubsection{2 - Measuring MAO Activity in Human Blood Platelets}

To assess the application of the modified kynuramine assay for measuring MAO activity in human tissue samples, a pilot study was conducted to measure the total MAO activity in blood platelets of smoking and non-smoking volunteers sourced from the Environmental Science and Research (ESR) Kenepuru Science Centre. The cohort comprised 16 non-smokers (5 males and 11 females), and 2 smokers (both female), ranging in age from 26 years to 59 years old. No attempts to recruit age or gender matched subject groups were made. Biometric data and a detailed smoking history were collected for all participants, and written informed consent was obtained from all subjects prior to their inclusion in the study. All procedures were approved by ESR's internal ethics committee, and by the Central Region Ethics Committee, New Zealand.

\section{$\underline{3.3-\text { RESULTS }}$}

\subsection{1 - Amplex Red ${ }^{\circledR}$ Assay (Invitrogen)}

\subsubsection{1 - Resorufin Standard Curve}

The resorufin reference standard was diluted over the concentration range from $0.5 \mu \mathrm{M}$ to 20 $\mu \mathrm{M}$ to construct a standard curve (Fig 7). Fluorescence from the diluted standards gave a sigmoidal-shaped standard curve over the concentration range tested, rather than the expected linear relationship. Fluorescence increased linearly at low concentrations of resorufin, up to $3 \mu \mathrm{M}$, before flattening out at concentrations above $5 \mu \mathrm{M}$. According to the 
manufacturer, the resorufin standard curve should be linear over the entire range of the assay, up to at least $20 \mu \mathrm{M}$. No explanation was found for the poor performance of the resorufin standard curve.

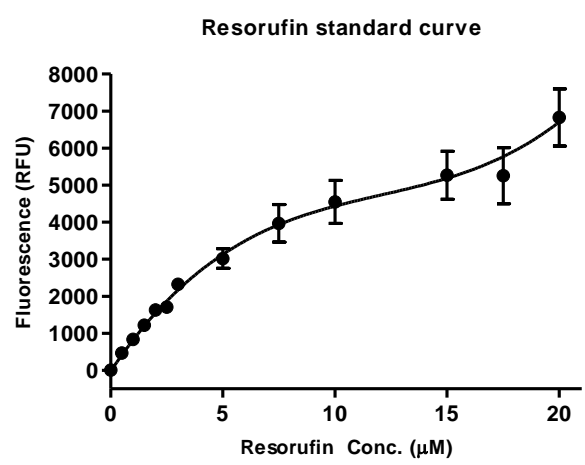

Figure 7: Resorufin standard curve. Fluorescence in relative fluorescence units (RFU) was measured at $590 \mathrm{nM}$ emission. Fluorescence versus resorufin concentration was linear for concentrations only up to approximately $5 \mu \mathrm{M}$. Each data point represents the mean \pm SEM of mean fluorescence from eight independent standard curves.

\subsubsection{2 - Using Amplex Red to Assess MAO Activity in Differentiated SH-SY5Y Cells}

Cultured SH-SY5Y cells were differentiated with retinoic acid (RA) for up to 10 days before testing with Amplex Red. Differentiation was assumed to have occurred, since the stellate $\mathrm{SH}$ SY5Y cells stopped dividing and extended long neurites from the cell bodies. The neurite processes were more evident and longer in cells exposed to RA for longer periods of time.

Total MAO activity in SH-SY5Y cells differentiated with $10 \mu \mathrm{M}$ retinoic acid

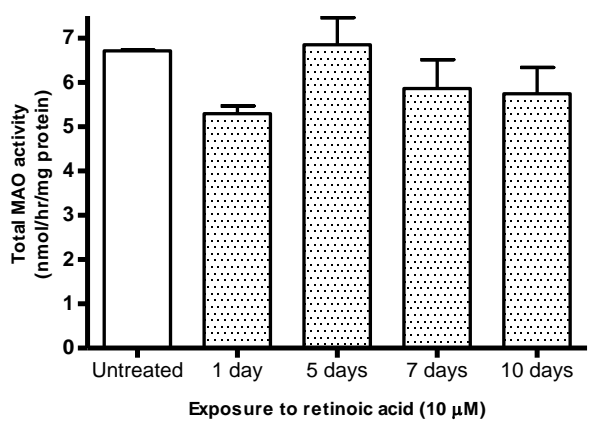

Figure 8: No significant difference in total MAO activity was observed in SH-SY5Y cells differentiated with $10 \mu \mathrm{M}$ retinoic acid for up to 10 days ( $\mathrm{n}=1$ sample tested in triplicate for each condition). 
Although cell morphology changed, this study found no significant differences in total MAO enzyme activity in SH-SY5Y cells following differentiation with retinoic acid for up to ten days (Fig. 8). However, this experiment was conducted successfully only once, as the Amplex Red assay gave very low MAO activity in other SH-SY5Y lysates tested. The reason for these subthreshold values in other lysates is not known.

\subsubsection{3 - Using Amplex Red to Distinguish MAO-A and MAO-B Activity in SH-SY5Y Cells}

The Amplex Red assay allows for the differentiation of MAO-A and MAO-B enzyme activities through the inclusion of the selective, irreversible inhibitors clorgyline and pargyline. Attempts were made to measure the contribution of MAO-A and MAO-B activity to the total MAO enzyme activity of SH-SY5Y cells, but unfortunately the results obtained were unreliable and inconsistent. Measures of MAO-A and MAO-B activities in SH-SY5Y lysates did not sum to the total MAO activity measured for that particular lysate (Fig. 9a). This suggests that cross inhibition was occurring; that is, clorgyline was partially inhibiting MAO-B, as well as MAO-A, and pargyline was partially inhibiting MAO-A as well as MAO-B. This experiment was repeated several times with both untreated SH-SY5Y cells and cells treated with RA. Measurements of total MAO activity were highly inconsistent between samples, and the measured MAO-A and MAO-B activities never summed to account for total MAO activity. The sum of the two enzyme activities was only about $60 \%$ of the total MAO activity measured in the absence of inhibitors.

(a)

Inhibition of MAO subtypes in SH-SY5Y cells by Amplex Red Assay Inhibitors

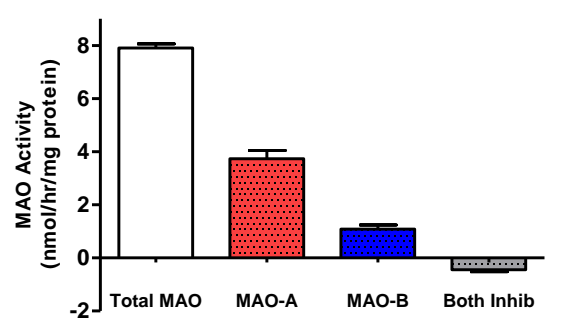

(b)

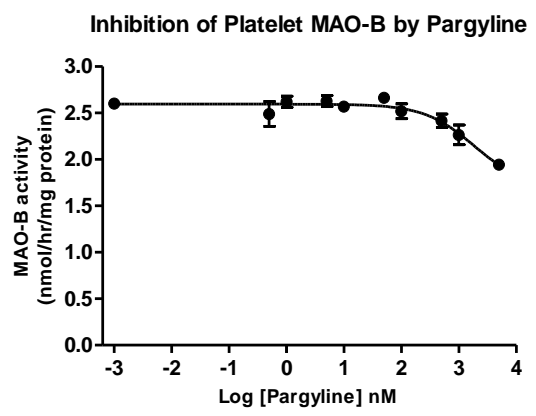

Figure 9: (a) Example measurements of total MAO, MAO-A, and MAO-B in SH-SY5Y cell lysate by including no inhibitor, $0.5 \mu \mathrm{M}$ pargyline, or $0.5 \mu \mathrm{M}$ clorgyline, respectively. MAO-A activity was measured as $3.7 \pm 0.31 \mathrm{nmol} / \mathrm{hr} / \mathrm{mg}$ protein, and MAO-B was measured as $1.1 \pm 0.16 \mathrm{nmol} / \mathrm{hr} / \mathrm{mg}$ protein. Total MAO measurement was $7.9 \pm 0.16 \mathrm{nmol} / \mathrm{hr} / \mathrm{mg}$ protein $(\mathrm{n}=1$ sample tested in triplicate for each condition). (b) MAO-B inhibitor pargyline did not completely inhibit MAO-B activity in human blood platelets, even at concentrations 10 times higher than recommended by the assay protocol. Each data point represents the mean \pm SEM of five replicate reactions tested in a single experiment. 
To investigate the efficacy of pargyline at inhibiting MAO-B enzyme activity, a sample of human blood platelets was tested with varying concentrations of pargyline. Platelets were chosen as the enzyme source for this experiment, as platelets are known to express only the MAO-B isotype (Thorpe et al., 1987). The Amplex Red assay protocol suggests using an inhibitor concentration of $0.5 \mu \mathrm{M}$, but this concentration inhibited platelet MAO-B activity by only $8 \%$ (Fig. 9b), and a pargyline concentration of $5 \mu \mathrm{M}$ inhibited MAO-B by only $27 \%$. This indicates either that the inhibitor included with the assay was ineffective at inhibiting MAO-B, or that background fluorescence in the reaction was very high. However, background fluorescence measured in the negative control which contained no $p$-tyramine substrate was generally very low, and this reagent background was deducted from the sample fluorescence before analysis.

\subsubsection{4 - Problems with the Amplex Red Assay}

A number of different experiments were conducted using Amplex Red to measure MAO enzyme activity in SH-SY5Y and U-118 MG cell lysates, or samples of human blood platelets, but very little usable data was obtained from these experiments. Most commonly, the Amplex Red assay failed to measure MAO activity in protein samples, despite proper precautions being taken to ensure the protein concentration was high enough and the enzyme remained active (Fig. 10).

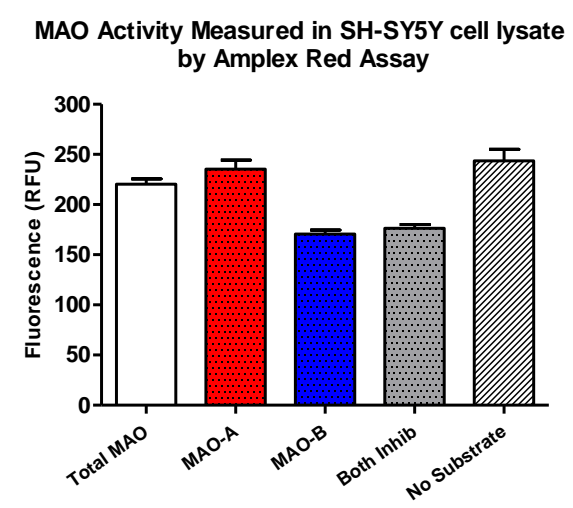

Figure 10: Example of a failed Amplex Red reaction testing for total MAO, MAO-A, and MAO-B activity in SH-SY5Y cells. No MAO activity was obtained, either due to low fluorescence in the samples, or high background fluorescence in the control without substrate. 
This problem was encountered when testing both SH-SY5Y lysates and samples of human blood platelets. As described above (section 3.3.1.3), the Amplex Red assay was also not able to adequately distinguish MAO-A and MAO-B enzyme activity in SH-SY5Y cells.

Efforts to optimize and improve the performance of the Amplex Red assay included attempts to measure MAO activity in SH-SY5Y and U-118 MG cells, and human blood platelets, and trials to determine effective inhibitor concentrations, but these were frequently thwarted by the poorly performing assay.

\subsection{2 - Kynuramine Assay}

\subsubsection{1 - 4-Hydroxyquinoline Standard Curve}

MAO-A and MAO-B enzymes will readily deaminate kynuramine to form 4-HQ, a product that fluoresces in the presence of excess base. A standard curve was constructed using dilutions of 4-HQ over a wide range (Fig. 11a, b). Fluorescence was linear over the entire concentration range of $4-\mathrm{HQ}$ standards tested, from $10 \mathrm{nM}$ to $10 \mu \mathrm{M}$.

(a)

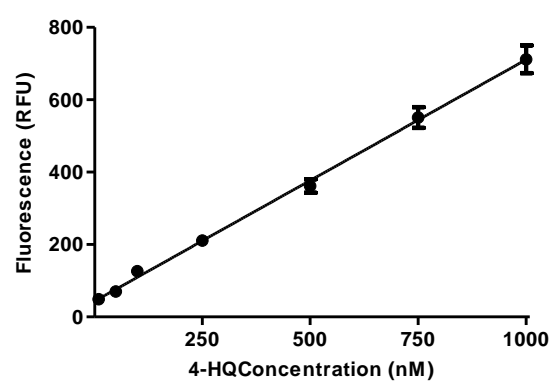

(b)

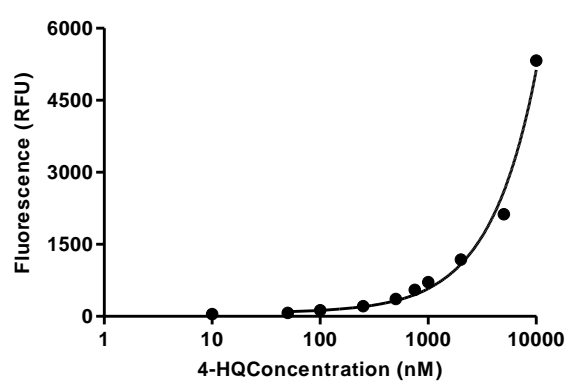

Figure 11: Standard curves of 4-HQ concentration and fluorescence at $380 \mathrm{~nm}$ emission. (a) 4-HQ concentration versus fluorescence is linear over the range of the assay, fitting the straight line equation $y=0.6603 x+44.028\left(R^{2}=0.9956\right)$. (b) A log plot of the same data shows an exponential curve, indicating the linear relationship is maintained for concentrations up to at least $10 \mu \mathrm{M} 4-\mathrm{HQ}\left(y=0.5058 x+72.893 ; R^{2}\right.$ $=0.9956)$. 


\subsubsection{2 - Determination of Optimum Substrate Concentration}

The kynuramine assay was tested by varying concentrations of the substrate to determine the optimum concentration for use in the standard reaction. Both SH-SY5Y lysates and preparations of purified recombinant MAO-A enzyme were tested.

When MAO activity was assessed in solutions of $10 \mu \mathrm{g} / \mathrm{mL}$ of purified MAO-A, the fluorescence increased nearly linearly at low concentrations of kynuramine (Fig, 12a). Fluorescence values reached a peak of approximately 1700 relative fluorescence units (RFU) at a concentration of $5 \mu \mathrm{M}$, and leveled off at higher kynuramine concentrations. In reactions using only $1 \mu \mathrm{g} / \mathrm{mL}$ of purified MAO-A, fluorescence values remained very low and did not increase with higher kynuramine concentrations, suggesting the enzyme was being saturated by substrate.

(a)

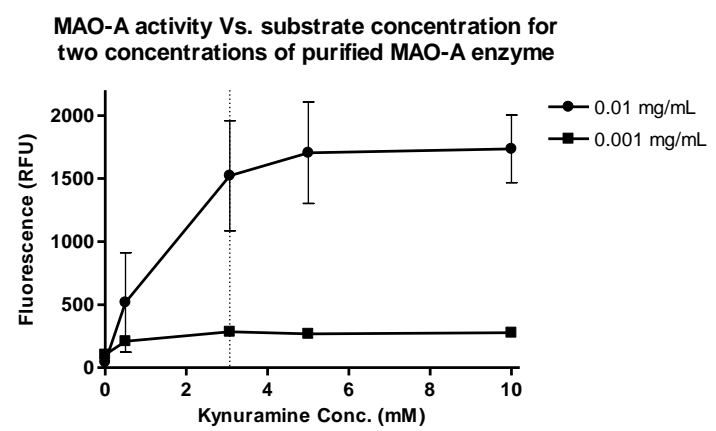

(b)

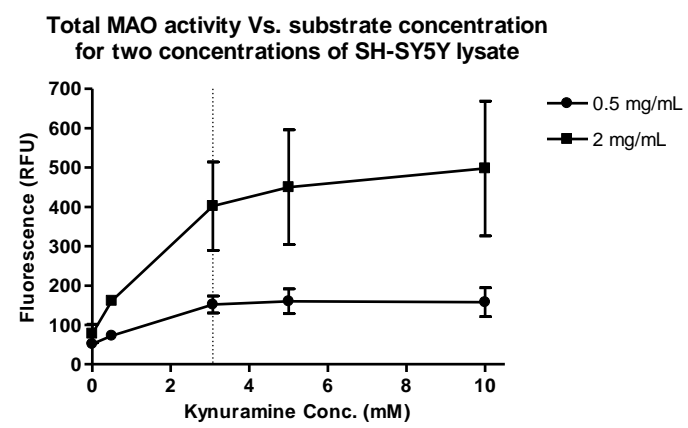

Figure 12: Kynuramine substrate concentrations were varied over the range $0.5 \mathrm{mM}-10 \mathrm{mM}$ and tested at different protein concentrations of (a) purified MAO-A enzyme and (b) SH-SY5Y lysate. Higher kynuramine concentrations gave increased fluorescence as expected. A substrate concentration of 3.07 $\mathrm{mM}$ kynuramine was selected for the standard assay based on the literature and supported by the above data (vertical dashed lines). Each data point represents the mean of two preparations, each assayed in triplicate ( $n=6$ wells).

Using $\mathrm{SH}-\mathrm{SY} 5 \mathrm{Y}$ lysates as the enzyme source gave similar results to those obtained with recombinant enzymes. At lysate total protein concentrations of $0.5 \mathrm{mg} / \mathrm{mL}$, fluorescence increased slightly at first, but leveled off at concentrations higher than $3.07 \mathrm{mM}$ kynuramine (Fig. 12b). Using $2 \mathrm{mg} / \mathrm{mL}$ of protein per reaction, fluorescence increased rapidly initially before slowing at kynuramine concentrations above $3.07 \mathrm{mM}$. Although fluorescence generation slowed considerably, the mean level of fluorescence increased over the range of kynuramine concentrations tested, but not in a linear manner as substrate approached saturating conditions. 
A kynuramine concentration of $3.07 \mathrm{mM}$ was selected for use in the standard assay, as in all experiments this concentration was situated on the curve in a region where fluorescence was increasing in response to higher substrate concentrations, indicating the enzyme was not reaching saturation. It was expected that using this concentration would give the most proportional RFU increase for an increase in MAO.

\subsubsection{3 - Fluorescence Increases Proportionally with Increased Enzyme Concentrations}

Protein lysates were prepared from cultured undifferentiated SH-SY5Y cells as described in section 3.2.1.1. The lysates were diluted over the range $0.5 \mathrm{mg} / \mathrm{mL}$ to $5 \mathrm{mg} / \mathrm{mL}$ of total protein. Each dilution was then tested for total MAO activity. Negative controls containing no protein showed background fluorescence of approximately 40 RFU. Increasing protein concentrations yielded higher fluorescence values (Fig. 13). The reaction seemed to reach saturation at $4-5 \mathrm{mg} / \mathrm{mL}$. Based on these results, protein concentrations of $2 \mathrm{mg} / \mathrm{mL}$ were selected for standard use in the assay, as this concentration was well within the linear range. Purified recombinant MAO-A was also tested, and fluorescence was found to increase proportionally with protein concentration.

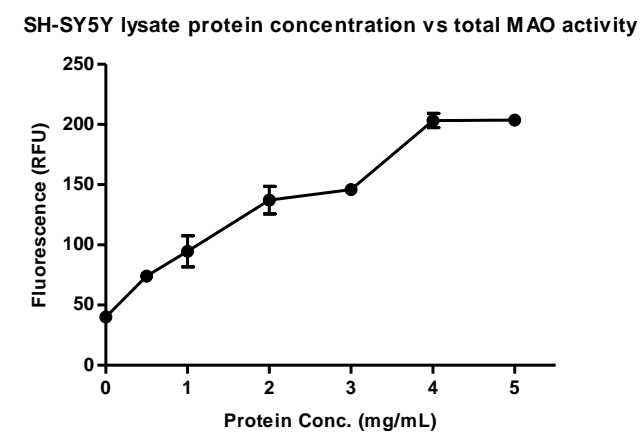

Figure 13: SH-SY5Y lysates were diluted to different protein concentrations, and then tested for MAO activity with $3.07 \mathrm{mM}$ kynuramine substrate. Fluorescence increased linearly with the amount of protein. Lysate concentrations of $2.0 \mathrm{mg} / \mathrm{mL}$ were used as a standard concentration for subsequent experiments. Each data point represents the mean fluorescence \pm SEM of at least 3 independent lysate preparations assayed in triplicate.

\subsubsection{4 - The Kynuramine Enzyme Reaction is Linear Over Time}

The behavior of the kynuramine reaction over time was tested using reactions containing 5 $\mu \mathrm{g} / \mathrm{mL}$ purified MAO-A and $2 \mathrm{mg} / \mathrm{mL}$ SH-SY5Y lysates. Due to the requirement that the MAO 
assay be suitable for high-throughput testing, a fast assay turn-around time was preferred. Therefore, samples were assayed with $3.07 \mathrm{mM}$ kynuramine for between 0 and 60 mins. In both SH-SY5Y and MAO-A samples, the reaction showed a linear relationship between enzyme activity and time (Fig. 14). Thus, the enzymatic reaction remained active over the full timeframe tested.

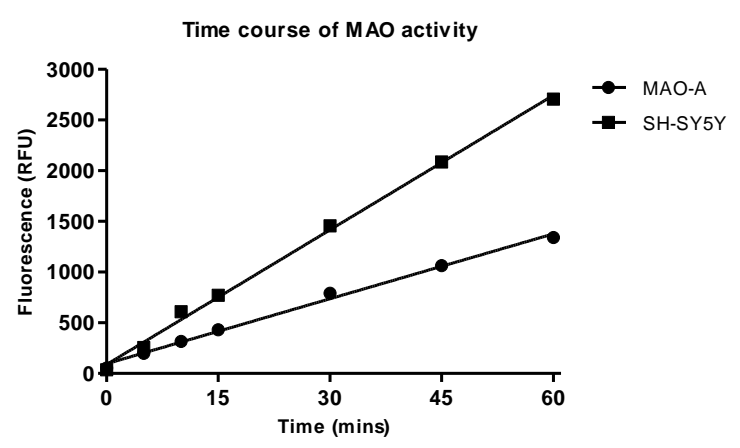

Figure 14: MAO activity was measured over time for purified MAO-A enzyme $(5 \mu \mathrm{g} / \mathrm{mL})$ and SH-SY5Y cell lysates $(2 \mathrm{mg} / \mathrm{mL})$. Both enzyme samples demonstrated a linear increase in 4-HQ product accumulation over a 60 min time-frame.

For the standard assay, reaction incubations of 15 or 30 mins were selected, as these incubation times yielded linear fluorescence accumulation that was significantly above baseline fluorescence.

\subsubsection{5 - Kynuramine Assay Variability}

The variability of the kynuramine assay was examined by assessing biological variation between samples and variation between replicate and repeat testing of SH-SY5Y lysates. To assess the between-sample variability of the kynuramine assay the spread of total MAO-A activities measured from independently tested, untreated $\mathrm{SH}-\mathrm{SH} 5 \mathrm{Y}$ cell lysates was examined. A high degree of variability was observed in total MAO activity measured in different SH-SY5Y preparations (Fig. 15a). Measurements of total MAO activity ranged from $2.36 \mathrm{nmol} / \mathrm{hr} / \mathrm{mg}$ protein to $19.6 \mathrm{nmol} / \mathrm{hr} / \mathrm{mg}$ protein, with a mean \pm standard deviation of $11.54 \pm 5.6$ $\mathrm{nmol} / \mathrm{hr} / \mathrm{mg}$ protein and a Coefficient of Variation of $48.7 \%$.

To assess the degree of variation due to the assay itself, three SH-SY5Y lysates were tested for total MAO activity in triplicate on consecutive occasions a day apart. Although there was large variation in the total MAO activity measured for different SH-SY5Y lysate preparations, 
triplicate testing of each sample showed very little variation. Additionally, repeat testing of each sample the following day was very consistent with the results of the earlier test for all samples (Fig. 15b). The results of these analyses indicate there is a high degree of biological variation in the SH-SY5Y cell lysates (between-sample variation), but very small variation inherent in the kynuramine assay (within-sample variation).

(a)

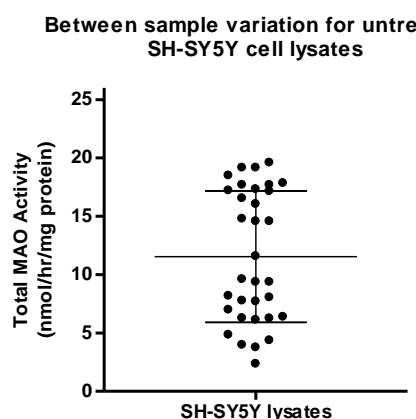

(b)

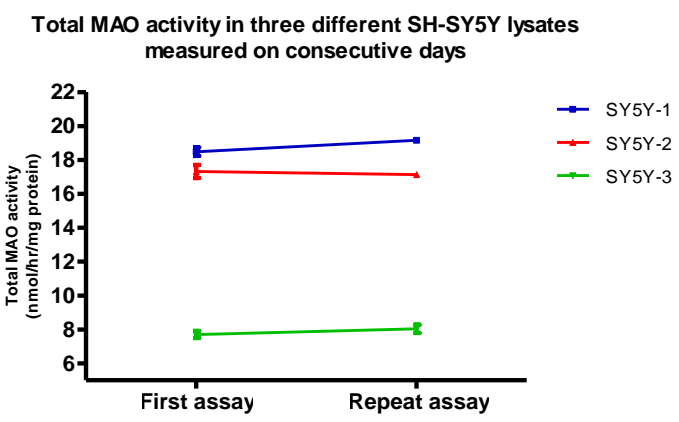

Figure 15: (a) Total MAO activity in SH-SY5Y cell lysates, measured by the kynuramine assay, was found to vary widely between independent lysate preparations. Error bars represent mean \pm standard deviation; $n=33$ independent lysates. (b) Three SH-SY5Y lysates which were tested for total MAO activity on consecutive days showed very little variation in total MAO activity on repeat testing. Data points represent the mean \pm SEM of each sample tested in triplicate.

\subsubsection{6 - Measuring MAO Enzyme Inhibition with Kynuramine}

To demonstrate that the modified kynuramine assay was suitable for measuring the inhibition of $\mathrm{MAO}$, the microplate procedure was used to determine the inhibition of purified human recombinant MAO-A and MAO-B enzymes by varying concentrations of the MAO-A selective inhibitor clorgyline and MAO-B selective inhibitor pargyline. Clorgyline inhibition is known to be approximately 1000 times more selective for MAO-A than for MAO-B, while the $K_{i}$ value of pargyline for MAO-B is eight times lower than that for MAO-A. Another commonly used MAO-B inihibitor, I-deprenyl shows greater selectivity for MAO-B than pargyline, with a $K_{i}$ forty times lower than the $K_{i}$ for MAO-A (C. J. Fowler et al., 1982). However; pargyline was used in these experiments because it was recommended and included in the Amplex Red assay kit. Although clorgyline and pargyline are reported to be selective inhibitors of MAO-A and MAO-B, some cross-inhibition occurs, and therefore the inhibition of both enzymes by each compound was tested. Inhibitor concentrations between $0.05 \mathrm{nM}$ and $10 \mu \mathrm{M}$ were used 
in these experiments (Fig. 16a - d). Non-linear regression analysis by GraphPad Prism estimated the $\mathrm{IC}_{50}$ of clorgyline for MAO-A to be $7.35 \mathrm{nM}$ (Fig. 16a), and the $\mathrm{IC}_{50}$ of pargyline for MAO-B to be $31.2 \mathrm{nM}$ (Fig. 16b).

As expected, much higher concentrations of pargyline and clorgyline were required to inhibit MAO-A and MAO-B, respectively, indicating the inhibitors were very selective for MAO-A or MAO-B. The IC $\mathrm{C}_{50}$ of pargyline for MAO-A was $209 \mathrm{nM}$ (Fig. 16c), and the IC $\mathrm{C}_{50}$ of clorgyline for MAO-B was 1207 nM (fig. 16d). Thus, clorgyline was 164 fold more effective against MAO-A, and pargyline was 6.7 fold more effective against MAO-B. All of the recorded $I C_{50}$ values for these inhibitors are similar to values previously reported in the literature (Table 6) (Lena et al., 1995).

(a)

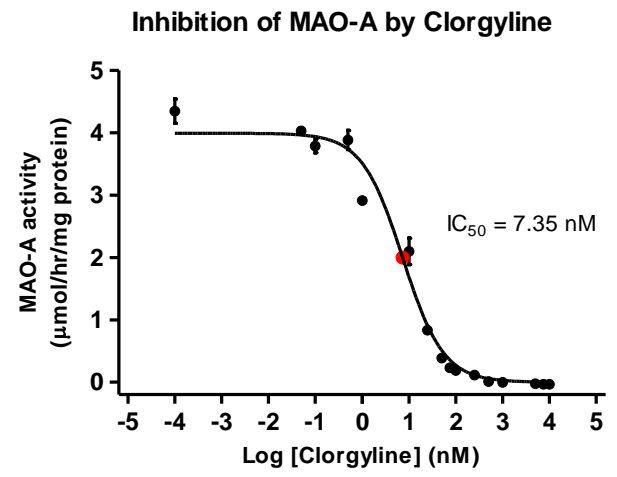

(c)

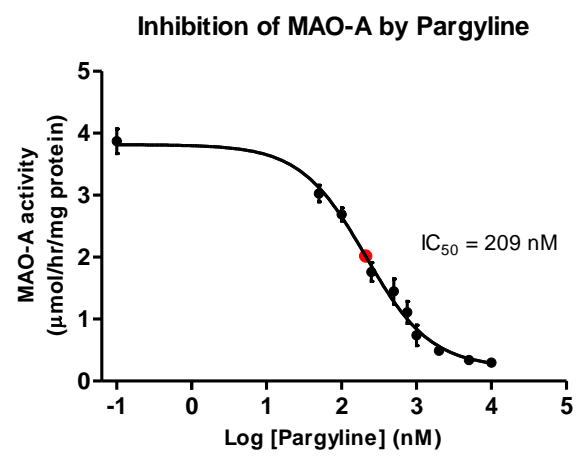

(b)

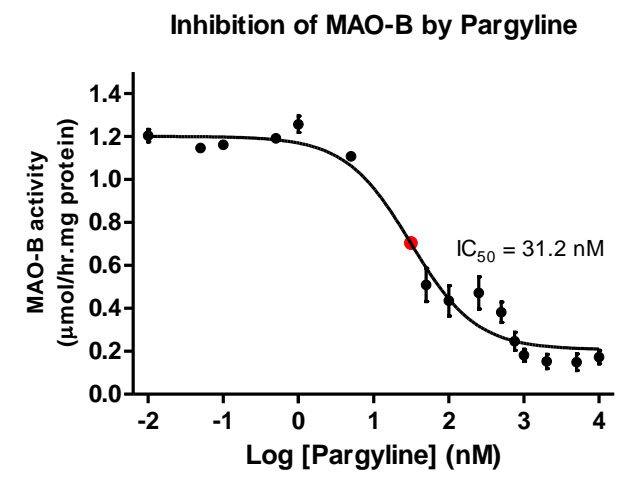

(d)

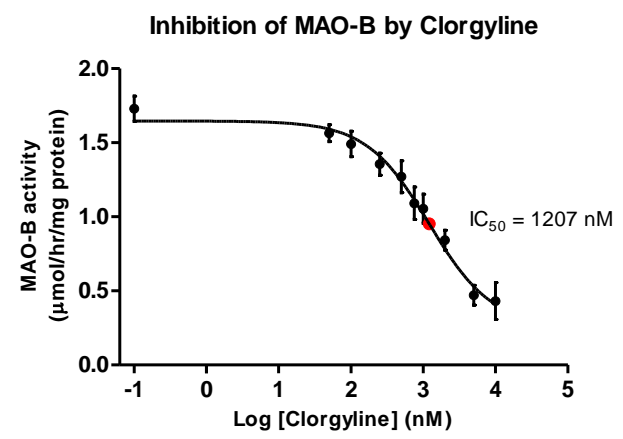

Figure 16: Dose-response plots of inhibition of purified recombinant human MAO-A and MAO-B enzymes by $(a, d)$ clorgyline and $(b, c)$ pargyline. Red data points show the position of $\mathrm{IC}_{50}$ concentrations. Each data point represents the mean \pm SEM of three reactions assayed in a single experiment. 
Table 6: $\mathrm{IC}_{50}$ Figures for clorgyline and pargyline inhibition

\begin{tabular}{lcccc} 
& \multicolumn{2}{c}{ MAO-A } & \multicolumn{2}{c}{ MAO-B } \\
& This study & Lena et al. (1995) & This study & Lena et al. (1995) \\
\hline Clorgyline & $7.35 \mathrm{nM}$ & $3.7 \mathrm{nM}$ & $1207 \mathrm{nM}$ & $2650 \mathrm{nM}$ \\
Pargyline & $209 \mathrm{nM}$ & $655 \mathrm{nM}$ & $31.2 \mathrm{nM}$ & $19.8 \mathrm{nM}$ \\
& & & & \\
\hline
\end{tabular}

Estimated $\mathrm{IC}_{50}$ values for MAO-A and MAO-B inhibition by clorgyline and pargyline in the present study are compared to values previously reported by Lena et al. (1995)

\subsubsection{7 - Measuring MAO Activity in Human Blood Platelets}

A pilot study was conducted to measure the total MAO activity in the blood platelets of a cohort of volunteers from Environmental Science and Research (ESR), Kenepuru Science Centre. Platelets are known to only express MAO-B activity, and therefore the total activity should reflect only this isotype (Thorpe et al., 1987). A high degree of variation was observed in measured platelet MAO activity between subjects. This was expected, as MAO activity is known to vary widely between individuals (Bagdy \& Rihmer, 1986; Snell et al., 2002). Female non-smokers had a mean platelet MAO-B activity of $80.2 \pm 4.6 \mathrm{nmol} / \mathrm{hr} / \mathrm{mg}$ protein; whereas, platelet samples from female smokers had a mean MAO-B of $75.9 \pm 12.2 \mathrm{nmol} / \mathrm{hr} / \mathrm{mg}$ protein, a difference that was not statistically significant. In contrast, male non-smokers had a mean platelet MAO-B enzyme activity of $48.9 \pm 12.9 \mathrm{nmol} / \mathrm{hr} / \mathrm{mg}$ protein, and this was significantly less than the MAO-B activity observed in female non-smokers (Fig. 17).

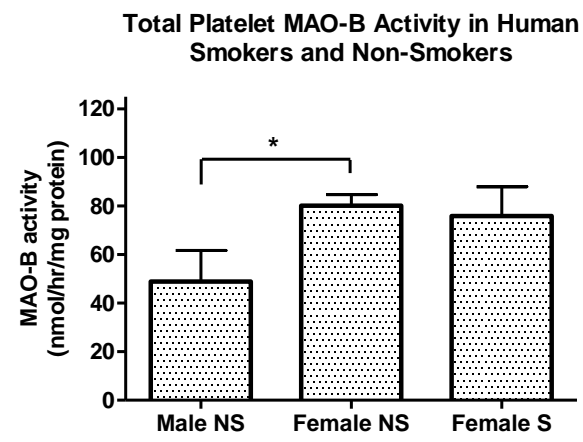

Figure 17: Total MAO activity in the blood platelets of non-smoking (NS) male volunteers $(n=5)$ was found to be significantly less than the activity in female non-smokers $(n=11)$. No difference was found between the platelet MAO activity of female smokers $(S)(n=2)$ and female non-smokers. (One way ANOVA with Bonferroni's multiple comparison post-test; * $\mathrm{P}<0.05$ ) 


\section{4 - DISCUSSION}

\subsection{1-Amplex Red Assay}

\subsubsection{1 - Measuring MAO Activity in Differentiated SH-SY5Y cells}

SH-SY5Y cells were differentiated using retinoic acid (RA) to determine whether differentiation had any effect on the expression of MAO enzymes. Testing of lysates prepared from differentiated cells with the Amplex Red Assay suggested that differentiation neither increased nor decreased total MAO expression in this cell line, although the experiment was successfully performed only once, due to the unreliable performance of the Amplex Red assay. Although a number of batches of cells were differentiated with RA for periods up to 14 days and tested with Amplex Red, no usable results were obtained due to high levels of background fluorescence.

A previous study by Ino et al. (1986) examined changes in MAO-A activity in SH-SY5Y cells differentiated with RA for up to 5 weeks. They found MAO-A activity did not significantly increase at any time point shorter than 5 weeks, and activity after 5 weeks of RA treatment increased MAO-A activity by 56\% (Ino et al., 1986). This suggests that the MAO activity was unlikely to have increased in the 7-day differentiation period used in this study. Since this study measured total MAO activity in undifferentiated SH-SY5Y by both Amplex Red and Kynuramine assays, differentiating the cells to achieve only a modest increase in enzyme activity was not deemed to be worthwhile. Furthermore, the experiments proposed for this study required many flasks of cells, and large numbers of cells were needed in each flask to generate protein lysates of sufficient total protein concentration for testing of MAO activity. Because cell differentiation involves the arrest of cell proliferation, treating cells with RA meant it was difficult to culture sufficient cell numbers for each experiment. Due to the difficulty and time required to culture adequate numbers of differentiated cells, and the preliminary evidence from this study that differentiation did not significantly alter MAO activity, the decision was made not to work with differentiated SH-SY5Y cells. Thus, undifferentiated cells were used for all subsequent experiments in this study.

\subsubsection{2 - Problems with the Amplex Red Assay}

The Invitrogen ${ }^{\circledast}$ Amplex Red Assay was found to be very problematic to use and very unreliable. The resorufin standard curve was non-linear, reactions frequently displayed high 
background fluorescence, or very low levels of fluorescence, and the inclusion of MAO inhibitors was unsuccessful at distinguishing MAO-A and MAO-B activity.

Problems encountered with the standard curve and inconsistent fluorescence suggests that the resorufin fluorescent product may not have been stable in the assay reactions used. The Invitrogen Amplex Red package insert advises that all kit reagents should be protected from light, and used promptly to avoid any degradation of the reagents. All appropriate precautions to ensure viability of the kit reagents were taken. The pack insert also stated that resorufin is unstable in the presence of thiol compounds, such as dithiothreitol (DTT) or $\beta$ mercaptoethanol, which are commonly used in cell lysate buffers. The present study specifically chose a cell lysate buffer (see Appendix 9.1) that did not contain these compounds to avoid any interactions with resorufin. Therefore, the cause of the inconsistent or unstable fluorescent product remains to be determined.

Problems distinguishing MAO-A and MAO-B through the use of selective inhibitors were likely due to the use of $p$-tyramine as a substrate for the reaction. The Amplex Red package insert recommends using benzylamine as a substrate when measuring MAO-B activity, and to use $p$ tyramine when measuring MAO-A or total MAO activity, since $p$-tyramine is a substrate for both enzyme isoforms. The present study measured total MAO activity, and attempted to determine the proportion of activity contributed by each isotype. To achieve this, $p$-tyramine was used for all reactions, since the reaction with benzylamine would have different kinetic parameters to $p$-tyramine, and it would be difficult to compare MAO-B activity measured using benzylamine to the total MAO activity measured with $p$-tyramine. Because $p$-tyramine was used as the substrate for all MAO reactions, concentrations of the MAO inhibitors clorgyline and pargyline needed to be carefully titrated to avoid any cross-inhibition. Reactions with SH-SY5Y cell lysates showed that at the $0.5 \mu \mathrm{M}$ inhibitor concentrations suggested by the Amplex Red package insert, a high degree of cross-inhibition occurred (Fig. 9a). Attempts to find inhibitor concentrations suitable to inhibit the selected isotype without inhibiting the other isotype were unsuccessful, due to the unreliable nature of this assay. A sample of human blood platelets was prepared and titrated against concentrations of pargyline in an attempt to determine the lowest pargyline concentration that would completely inhibit MAO-B activity (Fig. 9b). Unfortunately even the highest inhibitor concentrations were unable to eliminate all MAO activity in the platelet sample. This demonstrates either that pargyline was not a potent inhibitor of MAO-B, or that there were high levels of background or non-MAO-specific fluorescence in the samples tested. 
High levels of background or non-specific fluorescence in platelet preparations may be due to the activity of semicarbazide-sensitive amine oxidase (SSAO), another enzyme that metabolizes $p$-tyramine, producing $\mathrm{H}_{2} \mathrm{O}_{2}$ as a byproduct. SSAO is found ubiquitously in blood plasma and all vascularised tissues (O'Sullivan et al., 2004). The Amplex Red assay is known to detect activity of this enzyme, and SSAO is not inhibited by clorgyline or pargyline. Although reasonable efforts were made to isolate blood platelets from plasma, it is likely the platelet pellet had some plasma contamination. Although the Amplex Red kit claims to be suitable for measuring MAO activity in blood samples, it offers no solution for avoiding plasma contamination with SSAO enzyme activity.

Additionally, because Amplex Red assesses MAO enzymatic activity indirectly by measuring production of the $\mathrm{H}_{2} \mathrm{O}_{2}$ by-product, it is possible that other cellular processes that generate $\mathrm{H}_{2} \mathrm{O}_{2}$ were contributing to the high background fluorescence observed in SH-SY5Y lysates. $\mathrm{H}_{2} \mathrm{O}_{2}$ is generated by many different cellular mechanisms, and these would lead to false positive results or high background fluorescence. Ideally, an assay that is capable of measuring MAO activity by direct deamination of a substrate is preferred.

In summary, although the Invitrogen Amplex Red assay kit claims to provide a highly sensitive one-step method for the measurement of MAO enzyme activity, the present study found the assay to be highly unreliable and inconsistent. Brief attempts were made to optimize the assay, but these were unsuccessful and were finally abandoned due to the unreliability and high cost of the assay.

\subsection{2- Kynuramine Assay}

The kynuramine assay offers a number of considerable advantages over the previously tested Amplex Red assay. As discussed above, the Amplex Red assay measures MAO activity indirectly through the detection of $\mathrm{H}_{2} \mathrm{O}_{2}$ in an HRP-coupled reaction. In contrast, kynuramine is a substrate for both MAO-A and MAO-B, and enzyme activity is measured directly through the accumulation of $4-\mathrm{HQ}$, the fluorescent product of kynuramine deamination. The kynuramine reaction is less complex and is not subject to interference from other cellular processes that may generate $\mathrm{H}_{2} \mathrm{O}_{2}$. 
The experiments planned for this study required the testing of a large number of biological samples, and so a suitable high-throughput assay was needed. This experimenter found the Amplex Red assay to be time consuming to prepare due to a large number of reagent dilutions required in the assay protocol, and the long incubation time (1-3 hrs) of the reaction. Conversely, the kynuramine assay reaction tubes and micro-well plates were relatively simple to prepare, and the assay worked well with incubation times of 30 mins. Additionally, the cost of reagents for the kynuramine assay was almost 50 times less expensive than the per-test cost of the Amplex Red kit. Because the kynuramine assay was easier to set up, faster to run, and less expensive than the Amplex red kit, the kynuramine assay was more suitable for the high-throughput testing required for this study.

The most significant advantage of the kynuramine assay over Amplex Red was that the kynuramine assay proved to be much more reliable. While many problems were encountered with the Amplex Red assay that meant accurate measures of MAO activity could not be obtained, the kynuramine assay yielded MAO-dependent measures of fluorescence at all times. The reliability of the assay made the optimization of the assay for the standard microtube protocol, and adaptation and optimization of the micro-well plate protocol, reasonably straightforward and these measurements were able to be conducted in a timely manner. The kynuramine assay was then successfully used to measure total MAO activity in cultured cell lysates and human platelet samples and the dose-response of MAO inhibitors to purified recombinant enzymes. The results from these experiments demonstrated that the kynuramine assay is a robust, sensitive, and selective assay for MAO activity.

A significant advantage offered by the Amplex Red assay was its ability to distinguish MAO-A and MAO-B activity in cell lysates, although this study found the assay protocol to be unsuitable for this purpose. Unfortunately, the kynuramine assay cannot easily distinguish the activity of MAO-A and $-B$, since kynuramine is a substrate for both enzymes. It is possible that clorgyline and pargyline could be added to the kynuramine reactions to inhibit MAO-A and MAO-B, respectively. However, the concentrations of these inhibitors would need to be very carefully controlled to correctly inhibit the enzyme and avoid problems with crossinhibition. The present study chose not to attempt to optimize these inhibitor concentrations, as it was considered too complex and time consuming. Instead, the kynuramine assay was used to assess changes in total MAO activity in SH-SY5Y and U-118 MG cell homogenates. 


\subsubsection{1 - Measuring MAO Activity in Human Blood Platelets}

This study successfully demonstrated that the kynuramine assay could be used to measure MAO activity in human tissue samples, such as blood platelets. Total MAO activity in human platelets was determined and found to be significantly greater in non-smoking female volunteers than their non-smoking male counterparts. This is consistent with previously published findings of a number of studies that showed women display higher platelet MAO activity than men (Coccini et al., 2002; M. Harro et al., 2001; Snell et al., 2002). Snell et al. (2002) also reported that the MAO-B protein concentration in platelets from female subjects was significantly greater than in males. They found specific activity of the enzyme was no different between males and females; therefore, the increased activity in women was due to higher concentrations of enzyme in women's platelets.

A number of other studies have reported smokers to have lower MAO activity than nonsmokers (Berlin et al., 1995b; J. S. Fowler et al., 2003; J. S. Fowler et al., 1996a; J. S. Fowler et al., 1996b; Launay et al., 2009); however, this study found no difference in the total platelet MAO activity of female smokers compared to female non-smokers. No significant conclusions can be drawn from these data, as the cohort studied included only two female smokers.

\section{$\underline{3.4 .3-\text { Summary }}$}

This study initially trialed the Invitrogen Amplex Red MAO activity test kit for the measurement of MAO-A and MAO-B in cultured cell lysates. However, this assay was too unreliable, giving inconsistent and variable results, and was too costly to undertake extensive optimization experiments. In contrast, the kynuramine assay performed consistently, and proved to be sensitive and robust while being relatively inexpensive, but lacks easy differentiation of MAO-A and MAO-B isotype activity. 


\section{Chapter Four: Inhibition of MAO Enzyme Activity by Tobacco Extracts}

As discussed earlier (section 1.4), many studies have reported inhibition of MAO enzymes in a complex manner by tobacco compounds, and this has been tested over a number of different experimental paradigms. Many of these studies have examined the effects of tobacco on whole organisms. For example, Fowler et al. used PET scanning to determine that activity of MAO enzymes was reduced in the brains and peripheral organs of smokers compared to non-smokers (J. S. Fowler et al., 2003; J. S. Fowler et al., 2005; J. S. Fowler et al., 2000), and Gilbert et al. measured inhibition of MAO-B in blood platelets of smokers attempting to quit (Gilbert et al., 2003). These studies examined the gross effects of whole tobacco on very complex systems. Studies in humans and animal models have been very useful in identifying the overall consequences of tobacco exposure, but the inherent complexity in whole organism experiments means it can be difficult to tease out the exact nature of the MAO inhibition induced by tobacco compounds. These compounds are subject to many homeostatic and physiological mechanisms, which can mask or perturb the specific effects of tobacco compounds on MAO enzymes.

The effects of tobacco extract on MAO have been more closely examined in less complex experimental systems. For example, Yu \& Boulton (1987) used rat lung homogenates to determine that compounds in cigarette smoke and leaf extracts irreversibly inhibit MAO. Castagnoli et al. (2002), Mendez-Alvarez et al. (1997a), and Hauptman \& Shih (2001) demonstrated inhibition of MAO-A and MAO-B by tobacco compounds in rat brain mitochondrial preparations, and Khalil et al. (2000) used human mitochondrial preparations to assess the MAO inhibitory potential of a naphthoquinone compound isolated from tobacco leaves. While these experimental systems are less complex than whole organisms, many non-specific interactions are still possible due to the heterogeneous nature of the protein preparations used. Recently, purified human recombinant MAO-A and MAO-B enzymes have become commercially available, and these purified enzymes offer the opportunity to test tobacco extracts in a very simple 
experimental system. This allows examination of the direct inhibition of MAO-A and MAO-B by tobacco compounds in the absence of any confounding or interfering interactions, and this may give a clearer indication of the nature of MAO inhibition induced by tobacco extracts. For example, Herraiz \& Chaparro (2005) used purified MAO-A and MAO-B enzyme solutions to test the inhibitory potential of cigarette smoke solutions and found that tobacco smoke extracts inhibited MAO-A competitively and exhibited a mixed-type inhibition for MAO-B.

However, the interaction between TPM and MAO-A and MAO-B is likely to be quite complex. Smoking tobacco is formulated from the dried leaves of the tobacco plant and contains over 4000 different compounds. Many of these have yet to be characterized, and as described earlier (see section 1.4), a number of different compounds with MAO inhibitory action have been identified in tobacco smoke. Additionally, no two tobacco samples will be exactly the same, as the compounds present within the tobacco plant are subject to subtle changes and variations depending on the regional conditions, agricultural practices, and variety of the tobacco plant. Different methods of tobacco treatment and preparation also mean there can be quite significant differences in the composition of any given tobacco sample.

To date, relatively little research has examined the differences in composition between various types or brands of tobacco. A study of forty-eight brands of filtered cigarettes produced by Philip Morris USA Inc. compared differences in the mainstream smoke yield for over forty tobacco smoke constituents (Counts et al., 2004), and several other studies have examined differences in selected tobacco compounds. Rickert et al. (1985) compared the nicotine, carbon monoxide, and tar content of several brands of manufactured cigarettes, hand-rolled cigarettes, and cigars available on the Canadian market, finding that smoke tar and nicotine yields were greatest in cigars, followed by hand-rolled cigarettes and manufactured cigarettes, in that order. More recently, Calafat et al. (2004) examined the tar, nicotine, and carbon monoxide (CO) yields of mainstream cigarette smoke from 77 different cigarette brands purchased in 35 countries. They reported that mean tar, nicotine, and CO smoke yields varied widely between brands and also varied by region. Given that it has been recognized that there are inherent differences in the composition of tobacco smoke, it can be inferred that these composition differences may have an impact on the effects of different tobacco smokes. For example, while studies have examined the effects of cigarette smoke on the activity 
of MAO, it is unknown whether there are any observable brand differences in the degree of MAO inhibition. Furthermore, some cigarettes, such as Quest ${ }^{\circledR}$ nicotine-free cigarettes, are prepared from a genetically modified tobacco strains, and their composition is likely to differ significantly from standard cigarettes. An interesting question would be whether Quest ${ }^{\circledast}$ nicotine-free cigarettes exhibit the same MAO inhibitory activity as standard nicotine cigarettes.

While several studies have examined the effects of tobacco products on the activity of MAO enzymes, there has been little research into the influence of smoking cessation therapies on MAO. This is likely, in part, to be due to the fact that the most common smoking cessation therapies involve nicotine replacement therapy, and nicotine itself has been reported to have no effect on MAO enzyme activity (Oreland et al., 1981). One such cessation aid is the recently marketed RUYAN $^{\circledR}$ Electronic Cigarette.

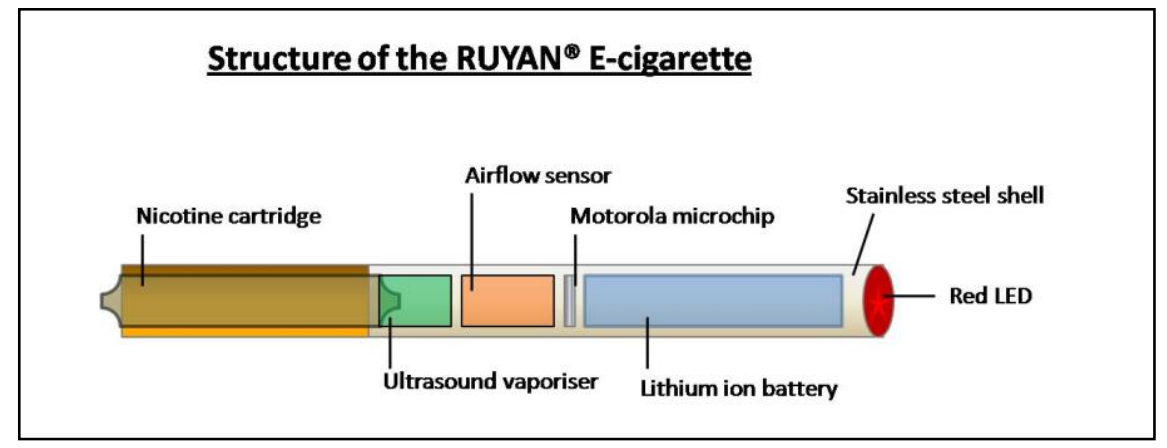

Figure 18: Component diagram of the E-cigarette. The cigarette body comprises a stainless steel shell housing a vaporizer unit, airflow sensor, microchip, and lithium ion battery. The nicotine cartridge provides nicotine and flavor compounds in a propylene glycol-based fluid. A red LED simulates the lit end of a normal tobacco cigarette.

The RUYAN E-Cigarette is a small battery-powered atomizer designed to simulate the look and feel of a real tobacco cigarette. The E-cigarette is supplied with disposable plastic cartridges that fit onto the E-cigarette and contain a propylene glycol-based nicotine solution that is atomized when the user inhales (Fig. 18). The manufacturer Ruyan Group (Holdings) Ltd claims the RUYAN E-cigarette is a clean alternative to smoking, as it delivers no tar, carbon monoxide, or cancer-causing chemicals (http://www.ecigarettes.cn, 2006-2009). However, the E-cigarette package insert ingredient list includes nicotine and an unspecified Kentucky tobacco extract, as well as several plant-derived oils. It is uncertain how the compounds contained in the RUYAN E- 
cigarette affect users of this device, or whether the tobacco extract contained in the cartridge formulation might influence MAO in a manner similar to standard tobacco. To date there have been few published studies conducted on the effects of the RUYAN Ecigarette, or its efficacy as a smoking cessation aid (Pauly et al., 2007). Bullen et al. (2010) reported that the Ruyan E-cigarette reduced study participants' desire to smoke and tobacco withdrawal symptoms to a degree similar to that seen with the Nicorette ${ }^{\circledR}$ nicotine inhalator (manufactured by Pfizer Health $A B$, Helsingborg, Sweden), but further research is required into the safety and efficacy of this device as a smoking cessation aid.

\subsection{2 - Objectives}

The present study investigated the inhibition of purified recombinant MAO enzymes in response to treatment with nicotine and a number of tobacco extracts. The aim of these experiments was to determine if TPM inhibited MAO-A and/or MAO-B in vitro, and to measure the extent of this inhibition. Several previous studies have demonstrated MAO inhibition by tobacco smoke extracts (Castagnoli et al., 2002; Herraiz \& Chaparro, 2005; Mendez-Alvarez et al., 1997a; P. H. Yu \& Boulton, 1987). The present study aimed to replicate some of the previously reported results using TPM extracts prepared from cigarette and tobacco products commercially available in New Zealand. It was hoped that these experiments would identify whether any difference or variation existed in MAO inhibitory activity between different brands and preparations of tobacco product. In addition, these experiments were designed to test whether non-nicotine tobacco components showed any MAO inhibitory activity. To test this, TPM derived from de-nicotinized and low-nicotine content cigarettes was tested for MAO inhibition as well.

The present study also examined the differences in the MAO inhibitory properties of TPM derived from the mainstream smoke of standard cigarettes compared to cigarettes handrolled from fine-cut tobacco. Eight popular brands of cigarette sold in New Zealand were compared with five brands of loose-leaf tobacco with regard to their nicotine yield, tar yield, and MAO-A and MAO-B inhibitory activity.

Samples taken from the RUYAN ${ }^{\circledR}$ Electronic Cigarette were also tested for MAO-A and MAO-B inhibitory activity, to determine if the RUYAN ${ }^{\circledR}$ has any effect on MAO, and to compare these samples with the inhibitory effects of complete tobacco TPM. 


\section{$\underline{4.2 \text { - MATERIALS AND METHODS }}$}

The activity of MAO-A and MAO-B enzymes was tested in response to exposure to a number of different tobacco and nicotine products. The activity of purified recombinant MAO-A and MAO-B enzymes following treatment with tobacco extracts was measured using the modified kynuramine micro-well plate assay. This assay was developed specifically for use in this study and is described in detail in Chapter Three of this thesis.

\subsection{1 - Purified Recombinant MAO Enzymes}

Purified human recombinant MAO-A and MAO-B enzymes were purchased from Sigma Aldrich (Cat. \# M7316 and M7441, respectively). These enzymes had been prepared in an insect baculovirus expression vector and purified. The purified enzyme was supplied as a $5.0 \mathrm{mg} / \mathrm{mL}$ protein solution in $100 \mathrm{mM}$ potassium phosphate, $\mathrm{pH}$ 7.4., $0.25 \mathrm{M}$ sucrose, 0.1 $\mathrm{mM}$ EDTA, and $5 \%$ glycerol. Vials of enzyme solution were shipped on dry ice from the supplier and stored at $-80^{\circ} \mathrm{C}$ to maintain enzyme activity. Enzyme stocks were thawed quickly and maintained on ice while $10 \mu \mathrm{L}$ aliquots were removed to microcentrifuge tubes. These aliquots were then stored at $-80^{\circ} \mathrm{C}$ until required.

\subsection{2 - Kynuramine Assay}

The modified kynuramine micro-well plate method described in section 2.4.2.1 was used to test the effect of purified nicotine and tobacco TPM extracts on the activity of MAO-A and MAO-B enzymes. Briefly, TPM extracts were diluted to relevant working concentrations with sodium phosphate buffer $(\mathrm{pH} 7.4)$, maintaining an ethanol concentration of $0.5 \%$ to control for any effects of the ethanol solvent. The diluted TPM solutions were pipetted in $100 \mu \mathrm{L}$ aliquots into the wells of a black polystyrene 96-well microplate in triplicate, before addition of $60 \mu \mathrm{L}$ of $0.307 \mathrm{mM}$ kynuramine dihydrobromide. The assay reaction was started with the addition of $40 \mu \mathrm{L}$ of $125 \mu \mathrm{g} / \mathrm{mL}$ human recombinant MAO-A or MAO-B enzyme. The microplates were then covered with aluminium foil to protect the reaction from light, and the plates were incubated in the dark at $37^{\circ} \mathrm{C}$ for 30 mins. The reaction was terminated by 
addition of $75 \mu \mathrm{L}$ of $2.5 \mathrm{M} \mathrm{NaOH}$ to each well, and the fluorescent products of the reaction were measured using an Optima fluorescence spectrophotometer at excitation and emission wavelengths of $320 \mathrm{~nm}$ and $380 \mathrm{~nm}$, respectively.

Relative fluorescence of the reactions was compared to the fluorescence of 4hydroxyquinoline reference standards that were diluted over the range of $10 \mathrm{nM}$ to $1 \mu \mathrm{M}$ and incubated in tandem with the test samples. Additionally, negative control wells that contained sodium phosphate buffer in place of MAO enzyme, in addition to diluted TPM and kynuramine were prepared and incubated with the reactions. The fluorescence data from these negative control wells was used to correct for any auto-fluorescence of the TPM extracts.

MAO-A inhibition was confirmed by testing the MAO-A activity concentration-response to clorgyline, a well-known MAO-A inhibitor, over the range of $1 \mathrm{pM}$ to $10 \mu \mathrm{M}$ clorgyline. Similarly, MAO-B inhibition was also confirmed by testing the concentration-response of the enzyme to the MAO-B inhibitor pargyline, using the same inhibitor concentration range as for clorgyline.

\subsection{3 - Tobacco Extract Exposures}

TPM filters were prepared by Labstat International, Inc. (Kitchener, Canada) as described in section 2.2.1. These filters were prepared from a range of different cigarettes and loose-leaf tobacco products, comprising eight different cigarette brands (Table 7) and five different loose-leaf tobacco brands (Table 8 ) that are commercially available for sale in New Zealand. Additionally, TPM filters were also prepared from low nicotine and nicotine-free Quest ${ }^{\circledast}$ cigarettes - Quest 1 and Quest 3, respectively. Quest nicotine-free cigarettes are prepared from tobacco strains that have been genetically modified to prevent the expression of nicotine. These cigarettes are not commercially available in New Zealand and were a gift from the manufacturer (Vector Tobacco Ltd., North Carolina, USA). The cigarette and tobacco samples were burnt and the smoke extracted and collected onto paper filter pads by smoking machines (Labstat, Canada. See section 2.2.1). Cigarette samples were collected at a ratio of between 13 and 20 cigarettes per filter, and filters were weighed before an after collection to determine the mass of tar 
collected. Loose-leaf tobacco samples were hand-rolled, using commercially available tobacco wrap papers and cellulose acetate filter tips, into cigarettes containing $900 \mathrm{mg}$ of tobacco. TPM from these hand-rolled cigarettes was then collected at a ratio of 6-7 cigarettes per paper filter pad. One TPM sample was collected from each of the brands tested, with the exception of the Holiday ${ }^{\circledR}$ Regular cigarette and the Park Drive tobacco products. For each of these products two samples were prepared from cigarettes or tobacco originating from different batches of the same brand.

After TPM collection by Labstat in Canada, the filters were sent to the ESR Kenepuru Science Centre with information about the extraction process, which included the number of cigarettes extracted per filter and the weighed mass of tar on each filter. On arrival each filter was catalogued with a unique sample number for tracking purposes (Appendix 9.2). Each filter was then extracted into ethanol, as described in section 2.2.1, in an effort to preserve as many volatile components in the tobacco smoke as possible. These extracts were then assayed for nicotine concentration using an Agilent 5973N gas chromatograph. The measured nicotine concentration and tar mass were then used to calculate the amount of nicotine and tar per cigarette, and these figures were compared to the manufacturers' reported data as printed on the packets of cigarettes and tobacco (Table 7 and Table 8, respectively).

The effect of ethanol on the enzymatic reaction was carefully controlled for, since previous testing had indicated that ethanol concentrations of greater than $0.5 \%$ significantly inhibited MAO enzyme activity (Y.-L. Lim \& P. Truman, Pers. Comms.). TPM concentrations used for testing were limited to a maximum of $0.25 \%$ ethanol in the final reaction to avoid this inhibition due to ethanol. Dilute absolute ethanol was added to the reactions when necessary to maintain a constant ethanol concentration of $0.25 \%$ in all reactions.

\subsection{4-Comparison of Nicotine, TPM, and Denicotinized TPM}

Initial enzyme experiments compared the effects of TPM from Quest nicotine-free cigarettes with TPM from Holiday Special Filter cigarettes. The latter brand was selected as representative of standard TPM. For these reactions the Holiday TPM (HTPM) sample was 
diluted to a final concentration of $0.2 \mu \mathrm{M}$ nicotine, and the Quest TPM (QTPM) sample was diluted to the same degree. These two samples were tested alongside purified nicotine and an ethanol vehicle control to determine the effects of these extracts on the activity of MAO-A and MAO-B.

Each experiment involved testing MAO-A or MAO-B activity in the presence of the ethanol control, $0.2 \mu \mathrm{M}$ purified nicotine, HTPM, and QTPM. Each condition was tested in triplicate. MAO-A enzyme activity in response to the test compounds was tested in 8 separate experiments, and MAO-B was tested over 7 experiments. Kynuramine fluorescence data from replicate reactions was averaged, and the mean values used in further analysis and calculations.

\subsection{5 - Comparison of MAO Inhibition Between Tobacco Products}

TPM samples from all cigarette and loose-leaf tobacco brands selected for this study were used to determine the concentration-response profile for MAO-A and MAO-B enzyme activity. Each TPM sample was tested for its effect on MAO inhibition at 7 dilutions over the range $0.0001 \%$ to $0.25 \%$ TPM, and each dilution was tested in triplicate. The data gathered in these experiments were analyzed with GraphPad Prism software, and a concentration-response curve was used to estimate the $\mathrm{IC}_{50}$ for every TPM sample tested, and the nicotine concentration at which MAO activity was inhibited by $50 \%$ was calculated for each TPM sample. This nicotine concentration was then used to compare any differences in MAO-A and MAO-B inhibition between different brands of cigarettes, different brands of loose-leaf tobacco, differences between cigarettes and loose-leaf tobacco, and differences between standard cigarettes and low-nicotine or nicotine-free Quest ${ }^{\circledR}$ cigarettes.

To validate that the calculated $I C_{50}$ values accurately represented the inhibitory properties of the test samples, each TPM sample was retested (in triplicate) for MAO-A inhibition at an arbitrary nicotine concentration ( $4 \mu \mathrm{M}$ nicotine). The degree of MAO-A inhibition measured in this experiment was then compared to the expected inhibition as predicted by the $I C_{50}$ and concentration-response curve data. Each TPM sample was then further tested for MAO-A inhibition at an arbitrary tar concentration $(0.01 \mathrm{mg} / \mathrm{mL})$, and 
the measured inhibition compared to the predicted inhibition at that tar concentration. These test procedures were used to provide data on the best way to estimate TPM concentrations between different samples.

\section{$\underline{\text { 4.2.6 - } \text { RUYAN }^{\circledR} \text { Cartridge Exposures }}$}

Samples of the nicotine solution in RUYAN ${ }^{\circledR}$ electronic cigarette cartridges were used to test the inhibitory activity of this product in a brief pilot study. Two RUYAN cartridges were received in sealed capsules from $\mathrm{Dr}$ M. Laugeson (Health New Zealand Ltd. Christchurch, NZ). These were marked with the known nicotine concentrations of 0 and $6 \mathrm{mg} / \mathrm{mL}$. Each cartridge was disassembled, and the solution-soaked glass wool filter was removed from the capsule. The solution was transferred to a clean, sterile plastic centrifuge tube, and the filter was carefully squeezed and centrifuged for 5 mins at $10,000 \times g$ to recover as much of the cartridge solution as possible.

Information from the manufacturer indicated that the cartridge solution contained nicotine and tobacco flavourings and extracts in a propylene glycol base. These solutions were diluted to working concentrations with distilled water before testing. The RUYAN 6 sample was diluted to final reaction concentrations of $0.2 \mu \mathrm{M}$ or $2 \mu \mathrm{M}$ nicotine, and the RUYAN 0 sample was equivalently diluted.

The RUYAN samples were tested for MAO-A and MAO-B inhibitory activity, initially at concentrations equivalent to $0.2 \mu \mathrm{M}$ nicotine and a reaction incubation time of 15 mins (7 experiments, each comprising triplicate reactions). Subsequently, the effects of the RUYAN 0 and RUYAN 6 samples on MAO-A and MAO-B were also examined at higher sample concentrations ( $2 \mu \mathrm{M}$ nicotine or equivalent; 4 experiments in triplicate), and for longer reaction durations (60 mins; three experiments in triplicate). Fluorescence data from replicate reactions were averaged, and the means used in further analysis. 


\section{$\underline{4.3-\text { RESULTS }}$}

\subsection{1 - Effect of Nicotine on MAO-A and MAO-B}

Purified recombinant MAO-A and MAO-B enzymes were incubated with a range of nicotine concentrations between $1 \mathrm{nM}$ and $10 \mu \mathrm{M}$ and assessed with the modified kynuramine assay. Neither MAO-A nor MAO-B activity was found to be significantly inhibited by nicotine at any of the concentrations tested. Very little variation was observed in enzyme activity between samples, and no concentration-response relationship with nicotine was observed (Fig. 19a, b).

(a)

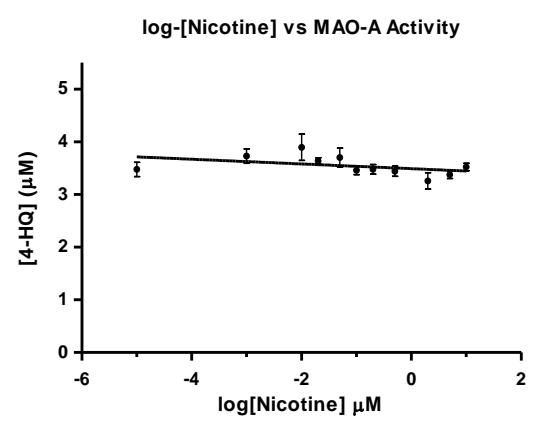

(b)

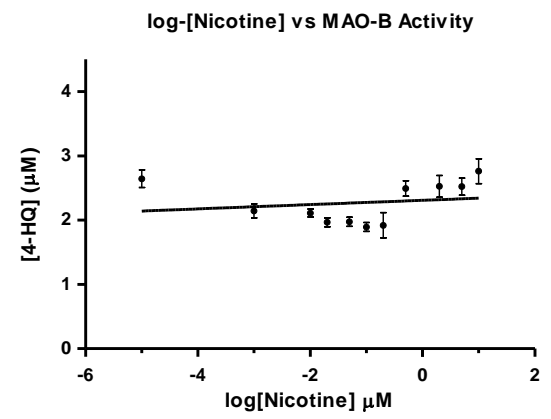

Figure 19: Purified recombinant MAO-A (a) and MAO-B (b) enzymes were treated with nicotine concentrations between $1 \mathrm{nM}$ and $10 \mu \mathrm{M}$, and the enzyme activity was measured by kynuramine assay. Neither MAO-A nor MAO-B were significantly inhibited by nicotine at any concentration tested. Each data point (mean \pm SEM) represents the mean enzyme activity from 3 experiments (5 replicates per experiment)

\subsection{2 - Comparison of Nicotine, TPM, and Denicotinized TPM}

The effect of total TPM on the activity of both MAO-A and MAO-B enzymes was assessed for TPM samples extracted from 11 different cigarette samples and 6 different loose-leaf tobacco samples (Tables 9 and 10). These covered a wide range of cigarette and tobacco products available commercially in New Zealand, as well as Quest 1 Low Nicotine and Quest 3 NicotineFree cigarettes.

Initially, the effects of standard TPM and nicotine-free TPM were tested on MAO-A and MAO$B$ at concentrations corresponding to $0.2 \mu \mathrm{M}$ nicotine. This concentration was considered physiologically relevant as nicotine concentrations have been reported to reach $0.2 \mu \mathrm{M}$ in the bloodstream of heavily dependent smokers (S. G. Gourlay \& N. Benowitz, 1997; J.E. Rose et 
al., 1999). TPM prepared from Holiday Special Filter cigarettes (HTPM) was chosen as representative of standard TPM, and this sample was compared to treatment with nicotine alone, or treatment with TPM extracted from Quest ${ }^{\circledR} 3$ Nicotine-Free cigarettes (QTPM). MAO-A activity was significantly inhibited by exposure to HTPM and QTPM at this concentration (Fig. 20a). In contrast, neither HTPM nor QTPM caused any significant reduction in MAO-B enzyme activity at this concentration (Fig. 20b).

(a)

MAO-A Activity following treatment with tobacco extracts

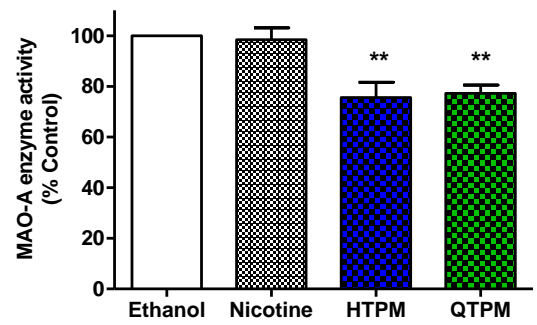

(b)

MAO-B Activity following treatment with tobacco extracts

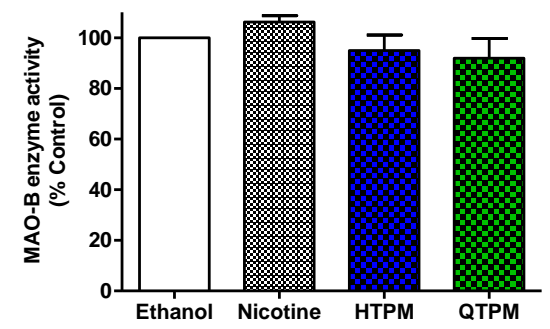

Figure 20: MAO-A (a) or MAO-B (b) enzyme activity was measured in the presence of purified nicotine, or TPM prepared from Holiday ${ }^{\circledR}$ brand cigarettes (HTPM), or Quest ${ }^{\circledR} 3$ Nicotine-Free cigarettes (QTPM). Nicotine had no measurable effect on the activity of either MAO-A or MAO-B enzyme. HTPM and QTPM samples inhibited MAO-A activity by $24.4 \%$ and $22.8 \%$, respectively, compared to nicotine alone or the ethanol control. These samples had no statistically significant effect on MAO-B activity. (One-way ANOVA with Bonferroni's multiple comparison test; $\mathrm{n} \geq 6$ experiments; ${ }^{* *} \mathrm{P}<0.05$ )

\subsection{3 - Comparison of Nicotine and Tar Yields Between Tobacco Products}

The TPM nicotine concentration and amount of tar per TPM filter were used to calculate the nicotine and tar yields per cigarette for manufactured cigarettes, and the tar:nicotine ratio for all samples (Tables $7 \& 8$ ). Tar and nicotine yields per cigarette were compared to the manufacturers' reported values for tar and nicotine yield printed on the outside of the cigarette packets. This comparison was not possible for the loose-leaf tobacco samples as the tobacco pouches were not printed with nicotine and tar content information.

A good agreement was found between the manufacturers' reported values and the actual nicotine and tar yield per cigarette. All cigarettes yielded less nicotine per cigarette than the maximum reported by the manufacturer. Most cigarettes yielded less measured tar than the maximum reported tar content, with the exception of Holiday cigarettes. Both the Holiday Special Filter and Holiday Regular brand cigarettes yielded more tar per cigarette than other 
brands, and these values exceeded the manufacturer's reported values of tar content by 1$10 \%$. However, the efficiency of the filter extraction process is unknown, and therefore the measured nicotine and tar content per cigarette in this study is likely to be a minimum estimate.

Table 7: Nicotine and tar data for cigarette brands tested

\begin{tabular}{|c|c|c|c|c|c|c|c|c|}
\hline \multirow[b]{2}{*}{ Brand } & \multicolumn{2}{|c|}{ Reported } & \multicolumn{3}{|c|}{ Filter data } & \multicolumn{3}{|c|}{ Calculated } \\
\hline & $\begin{array}{l}\text { Nicotine } \\
\text { (mg/cig) }\end{array}$ & $\begin{array}{c}\text { Tar } \\
\text { (mg/cig) }\end{array}$ & Cig/filter & $\begin{array}{l}{[\mathrm{Nic}]} \\
\text { (mg) }\end{array}$ & $\begin{array}{c}\text { Tar/filter } \\
\text { (mg) }\end{array}$ & $\begin{array}{l}\text { Nicotine } \\
\text { (mg/cig) }\end{array}$ & $\begin{array}{c}\text { Tar } \\
\text { (mg/cig) }\end{array}$ & Tar:Nic \\
\hline Winfield King Size Filter & $<1.5$ & $<16$ & 13 & 11.13 & 199.6 & 0.86 & 15.35 & 17.94 \\
\hline Winfield Extra Mild & $<1.2$ & $<12$ & 17 & 11.14 & 186.1 & 0.66 & 10.95 & 16.70 \\
\hline Benson \& Hedges Special Filter & $<1.5$ & $<16$ & 13 & 9.15 & 181.6 & 0.70 & 13.97 & 19.84 \\
\hline Pall Mall & $<1.5$ & $<16$ & 13 & 9.32 & 192.5 & 0.72 & 14.81 & 20.65 \\
\hline Holiday Special Filter & $<1.2$ & $<16$ & 12 & 7.21 & 210.8 & 0.60 & 17.57 & 29.23 \\
\hline Holiday Menthol Mild & $<0.8$ & $<12$ & 17 & 10.27 & 206.2 & 0.60 & 12.13 & 20.07 \\
\hline Rothmans King Size & $<1.5$ & $<16$ & 21 & 9.93 & 194.1 & 0.76 & 14.93 & 19.54 \\
\hline Holiday Regular (1) & $<1.5$ & $<16$ & 20 & 20.02 & 332.1 & 1.00 & 16.61 & 16.59 \\
\hline Holiday Regular (2) & $<1.5$ & $<16$ & 20 & 18.40 & 324.4 & 0.92 & 16.22 & 17.63 \\
\hline \multicolumn{6}{|c|}{ Mean ( \pm SEM) } & $0.76 \pm 0.05$ & $14.73 \pm 0.71$ & $19.8 \pm 1.28$ \\
\hline Quest 1 - Low Nicotine & $<0.6$ & $<10$ & 20 & 6.81 & 187.8 & 0.34 & 9.39 & 27.56 \\
\hline Quest 3- Nicotine Free & $<0.05$ & $<10$ & 20 & 0.57 & 163.4 & 0.03 & 8.17 & 285.90 \\
\hline
\end{tabular}

Data for tar and nicotine yields of TPM derived from commercially available cigarettes, including information reported on the cigarette packets by the manufacturer and measurements of tar and nicotine concentration from the ethanolextracted TPM samples. Values for nicotine yield per cigarette, tar yield per cigarette, and tar:nicotine ratio were calculated from the filter data. Holiday ${ }^{\circledR}$ brand cigarettes appeared to have higher tar/cigarette yields than other brands, and these slightly exceeded the manufacturers' reported values (bold values).

The tar:nicotine ratios were compared between manufactured cigarettes and loose-leaf tobacco cigarettes. Quest ${ }^{\circledR}$ cigarettes were omitted from the comparison as these were known to be significantly different from standard cigarettes with respect to nicotine content. Loose-leaf tobacco cigarettes had a mean tar:nicotine ratio of $27.9 \pm 1.7(n=6)$, which was significantly higher than manufactured cigarettes which had a mean tar:nicotine ratio of 19.8 $\pm 1.3(n=9)$ (Fig. 21). 
Table 8: Nicotine and tar data for loose-leaf tobacco brands tested

\begin{tabular}{|c|c|c|c|c|}
\hline \multirow[b]{2}{*}{ Brand } & \multicolumn{3}{|c|}{ Filter data } & \multirow{2}{*}{$\begin{array}{c}\text { Calculated } \\
\text { Tar: Nic }\end{array}$} \\
\hline & Cig/filter & {$[\mathrm{Nic}](\mathrm{mg})$} & Tar/filter (mg) & \\
\hline Port Royal & 7 & 7.95 & 217.5 & 27.38 \\
\hline Park Drive (1) & 8 & 8.45 & 259.6 & 30.72 \\
\hline Park Drive (2) & 7 & 9.23 & 235.7 & 25.53 \\
\hline Drum & 8 & 7.83 & 266.4 & 34.04 \\
\hline Holiday & 6 & 9.05 & 198.5 & 27.86 \\
\hline Holiday Menthol & 8 & 8.57 & 238.7 & 21.94 \\
\hline & & & Mean $( \pm$ SEM & $27.91 \pm 1.70$ \\
\hline
\end{tabular}

$\overline{T a r}$ and nicotine concentration data for TPM samples derived from loose-leaf roll-your-own tobacco. No manufacturer information regarding these parameters was available.

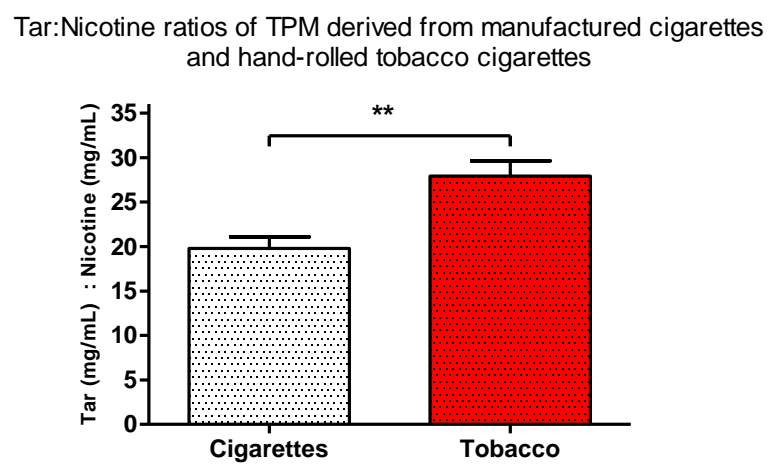

Figure 21: The mean tar:nicotine ratio from TPM samples from the six loose-leaf tobacco samples was significantly higher than TPM from the nine samples of manufactured cigarettes (Student's t-test, ** $\mathrm{P}<$ $0.01)$.

\subsection{4-Comparison of MAO Inhibition Between Tobacco Products}

\subsubsection{1 - MAO Concentration-Response for TPM Samples}

The concentration-response relationship for inhibition of both MAO-A and MAO-B was examined using the kynuramine microplate method for all TPM samples. These data were used to plot concentration-response curves for each sample, and the $\mathrm{IC}_{50}$ values for MAO-A and MAO-B inhibition were estimated using GraphPad Prism software. All TPM samples showed similar inhibition of MAO-A and MAO-B, and the TPM concentration-response curves for each sample all appeared very similar (Fig. 22a, b). 
The $I C_{50}$ value for each sample was expressed as a percentage of TPM dilution. However, this unit format is not very useful for comparison between samples as the TPM solutions were prepared from different numbers of cigarettes.

(a)

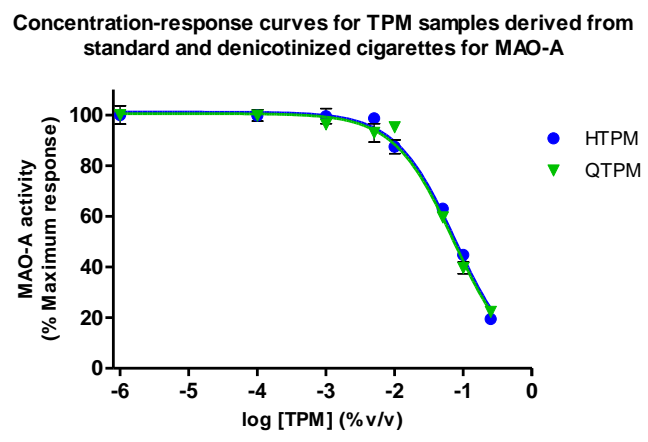

(b)

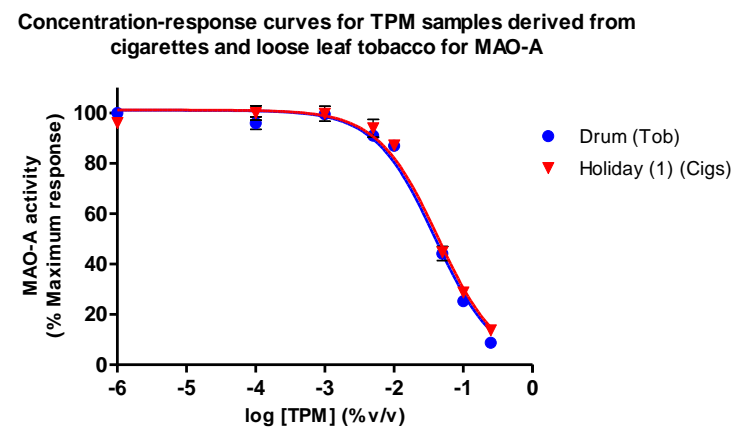

Figure 22: The concentration-response relationship for TPM inhibition of MAO-A was tested for all TPM extracts used. All TPM samples showed similar inhibition of MAO-A. (a) The concentration-response curves obtained for MAO-A inhibition by HTPM and QTPM. (b) The concentration-response curves for Drum $^{\circledR}$ loose-leaf tobacco and Holiday ${ }^{\circledR}$ cigarettes. (Tob = loose-leaf tobacco; Cigs = manufactured cigarettes.) Concentration is expressed as \% TPM dilution (v/v).

Using the known nicotine concentrations of each TPM sample, the $\mathrm{IC}_{50}$ value was then used to calculate the concentration of nicotine in the TPM sample when MAO-A and MAO-B activities are inhibited by $50 \%$ (Tables 9 and 10, respectively). These figures varied quite widely - from $1.42 \mu \mathrm{M}$ to $6.26 \mu \mathrm{M}$ nicotine for MAO-A inhibition, and from $2.07 \mu \mathrm{M}$ to $7.57 \mu \mathrm{M}$ nicotine for MAO-B inhibition. It should be noted that the nicotine concentration at the $\mathrm{IC}_{50}$ is intended to represent a standardized measure of the TPM concentration, and not strictly of the nicotine concentration. $\mathrm{IC}_{50}$ nicotine concentrations tended to be higher for MAO-B than MAO-A, suggesting that higher concentrations of TPM are required to inhibit MAO-B, in agreement with earlier data, presented in section 4.3.2. 
Table 9: MAO-A IC ${ }_{50}$ values of TPM samples and corresponding nicotine concentrations

\begin{tabular}{llcccc}
\hline & \multicolumn{1}{c}{$I_{50}$} & {$[$ Nic] @ } & \multicolumn{2}{c}{$\%$ Inhib at $4 \boldsymbol{\mu M}$ [Nic] } \\
Brand & $(\%$ TPM) & $I_{50}(\boldsymbol{\mu M})$ & Predicted & Measured \\
\hline Cigarette & Winfield King Size Filter & 0.069 & 3.64 & 51.8 & $52.3 \pm 1.1 \%$ \\
& Winfield Extra Mild & 0.075 & 3.95 & 50.3 & $46.6 \pm 0.8 \%$ \\
Benson \& Hedge Special Filter & 0.079 & 3.44 & 53.8 & $58.9 \pm 0.4 \%$ \\
Pall Mall & 0.085 & 3.74 & 51.7 & $55.1 \pm 0.7 \%$ \\
Holiday Special Filter & 0.077 & 2.65 & 60.2 & $61.9 \pm 1.0 \%$ \\
Holiday Menthol Mild & 0.094 & 4.55 & 46.8 & $56.3 \pm 1.6 \%$ \\
Rothmans King Size & 0.073 & 3.45 & 53.7 & $50.4 \pm 0.8 \%$ \\
Holiday Regular (1) & 0.047 & 4.43 & 47.4 & $44.4 \pm 1.0 \%$ \\
Holiday Regular (2) & 0.072 & 6.26 & 39.0 & $55.1 \pm 1.1 \%$ \\
Tobacco & Port Royal & 0.065 & 2.46 & 61.9 & $65.5 \pm 1.2 \%$ \\
Park Drive (1) & 0.044 & 1.76 & 69.5 & $75.4 \pm 0.3 \%$ \\
Park Drive (2) & 0.057 & 2.50 & 61.5 & $68.9 \pm 0.6 \%$ \\
Drum & 0.040 & 1.49 & 72.9 & $75.6 \pm 0.7 \%$ \\
Holiday Menthol & 0.035 & 1.42 & 73.8 & $73.3 \pm 0.6 \%$ \\
Holiday & 0.033 & 1.43 & 67.3 & $69.2 \pm 0.9 \%$ \\
\hline
\end{tabular}

Measured $\mathrm{IC}_{50}$ values for TPM samples (\% TPM $(\mathrm{v} / \mathrm{v})$ ) were used to calculate the nicotine concentration at the $I_{50}$, and the concentration-response curve was used to predict MAO-A inhibition at $4 \mu \mathrm{M}$ nicotine. This was compared to the actual MAO-A inhibition at $4 \mu \mathrm{M}$ nicotine measured experimentally (\% inhibition).

Using the nicotine concentration at the $\mathrm{IC}_{50}$ to quantify TPM is not very useful since the tobacco products from which the TPM samples were extracted vary in their nicotine content. To determine whether the estimated $I_{50}$ values accurately represented the inhibitory action of each TPM sample, the $\mathrm{IC}_{50}$ data were used to calculate the expected MAO-A inhibition at concentrations of TPM normalized to contain $4 \mu \mathrm{M}$ nicotine and these predicted values were then tested experimentally (Table 9). The predicted values of MAO-A inhibition were a good indicator of actual MAO-A inhibition, showing a mean difference from the measured \% inhibition of only $3.7 \%$ and a very significant correlation (Spearman coefficient $=0.90$, $P<0.0001)$. This confirms that the $I C_{50}$ values derived from the TPM concentration-response curves reflect the relationship between TPM concentration and MAO-A inhibition with reasonable accuracy. 
Table 10: MAO-B IC 50 values of TPM samples and corresponding nicotine concentrations

\begin{tabular}{|c|c|c|c|c|c|}
\hline Cigarette Brand & $\begin{array}{c}I_{50} \\
(\% \text { TPM) }\end{array}$ & $\begin{array}{l}{[\mathrm{Nic}] @} \\
I_{50}(\mu \mathrm{M})\end{array}$ & Tobacco Brand & $\begin{array}{c}I_{50} \\
(\% \text { TPM) }\end{array}$ & $\begin{array}{l}{[\mathrm{Nic}] @} \\
I C_{50}(\mu \mathrm{M})\end{array}$ \\
\hline Winfield King Size Filter & 0.082 & 4.30 & Port Royal & 0.091 & 3.44 \\
\hline Winfield Extra Mild & 0.129 & 6.82 & Park Drive (1) & 0.052 & 2.07 \\
\hline Benson \& Hedge Special Filter & 0.097 & 4.23 & Park Drive (2) & 0.059 & 2.57 \\
\hline Pall Mall & 0.088 & 3.89 & Drum & 0.077 & 2.87 \\
\hline Holiday Special Filter & 0.089 & 3.03 & Holiday Menthol & 0.085 & 3.44 \\
\hline Holiday Menthol Mild & 0.144 & 7.00 & Holiday & 0.090 & 3.88 \\
\hline Rothmans King Size & 0.122 & 5.75 & & & \\
\hline Holiday Regular (1) & 0.080 & 7.57 & & & \\
\hline Holiday Regular (2) & 0.060 & 5.27 & & & \\
\hline
\end{tabular}

Measured $\mathrm{IC}_{50}$ values for TPM samples were used to calculate the nicotine concentration at which MAO-B was inhibited by $50 \%$.

When the TPM samples were normalized to a standard nicotine concentration of $4 \mu \mathrm{M}$, a significant difference in MAO-A inhibition was observed between TPM samples derived from manufactured cigarettes and samples derived from loose-leaf tobacco cigarettes (Fig. 23). TPM extracts from manufactured cigarettes inhibited MAO-A by 55\%; whereas, TPM from rollyour-own cigarettes inhibited MAO-A by $71 \%$, a difference that was found to be highly statistically significant $(P<0.0001, n=6)$.

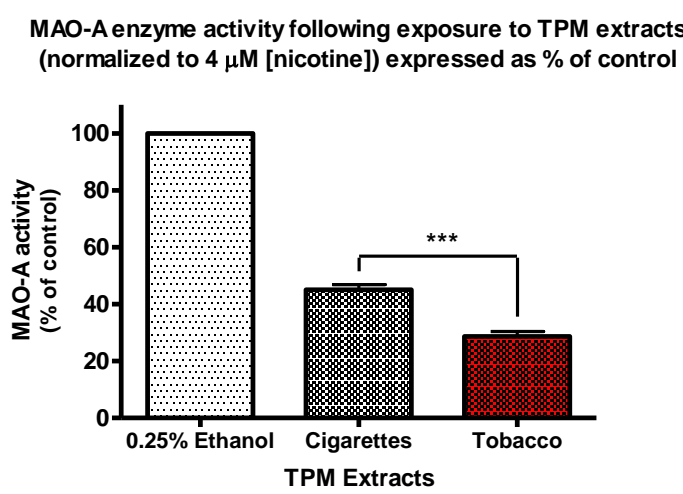

Figure 23: A significant difference was observed in the inhibition of MAO-A by TPM derived from factorymade cigarettes and roll-your-own tobacco products when TPM samples were normalized to a standard nicotine concentration of $4 \mu \mathrm{M}$. TPM derived from cigarettes inhibited MAO-A by $55 \pm 1.8 \%$ ( $n=9$ samples tested in triplicate), and loose-leaf tobacco TPM inhibited MAO-A by $71 \pm 1.7 \%$ ( $n=6$ samples tested in triplicate). This difference was found to be statistically significant (One-way ANOVA with Bonferroni's multiple comparison post-test; ${ }^{* * *} \mathrm{P}<0.0001$ ). 
Since TPM from loose-leaf tobacco cigarettes had a significantly lower nicotine yield compared to manufactured cigarette TPM (Fig. 21a), but no significant difference was observed in tar yield between both types of tobacco product, MAO-A inhibitors were assumed to be in the tar fraction of TPM, since neither ethanol nor nicotine directly inhibits MAO-A. It was thought that normalizing the nicotine concentration between samples resulted in looseleaf tobacco TPM reactions having a greater quantity of tar in the reaction than the TPM from manufactured cigarettes. If the loose-leaf tobacco samples had greater quantities of tar, and thus more MAO-A inhibitors, this would explain why these samples inhibited MAO-A much more effectively than the manufactured cigarette TPM.

Table 11: Tar concentrations in TPM samples

\begin{tabular}{|c|c|c|c|c|}
\hline & \multirow[b]{2}{*}{ Brand } & \multirow{2}{*}{$\begin{array}{c}\text { [Tar] @ } \\
I C_{50}(\mathrm{mg} / \mathrm{mL})\end{array}$} & \multicolumn{2}{|c|}{$\%$ Inhib at $0.01 \mathrm{mg} / \mathrm{mL}$ [Tar] } \\
\hline & & & Predicted & Measured \\
\hline \multirow[t]{9}{*}{ Cigarette } & Winfield King Size Filter & 0.0106 & 48.0 & $43.1 \pm 0.1 \%$ \\
\hline & Winfield Extra Mild & 0.0107 & 48.3 & $48.5 \pm 1.7 \%$ \\
\hline & Benson \& Hedge Special Filter & 0.0111 & 47.5 & $49.4 \pm 1.3 \%$ \\
\hline & Pall Mall & 0.0125 & 44.4 & $47.9 \pm 1.1 \%$ \\
\hline & Holiday Special Filter & 0.0125 & 44.4 & $42.5 \pm 3.5 \%$ \\
\hline & Holiday Menthol Mild & 0.0148 & 38.3 & $44.4 \pm 1.4 \%$ \\
\hline & Rothmans King Size & 0.0109 & 47.8 & $51.2 \pm 1.2 \%$ \\
\hline & Holiday Regular (1) & 0.0119 & 45.6 & $59.3 \pm 2.3 \%$ \\
\hline & Holiday Regular (2) & 0.0179 & 35.8 & $36.9 \pm 1.8 \%$ \\
\hline \multirow[t]{6}{*}{ Tobacco } & Port Royal & 0.0109 & 47.8 & $44.3 \pm 3.2 \%$ \\
\hline & Park Drive (1) & 0.0087 & 53.3 & $59.2 \pm 1.2 \%$ \\
\hline & Park Drive (2) & 0.0103 & 49.1 & $58.2 \pm 0.9 \%$ \\
\hline & Drum & 0.0082 & 54.9 & $50.6 \pm 1.5 \%$ \\
\hline & Holiday Menthol & 0.0064 & 60.9 & $44.3 \pm 0.6 \%$ \\
\hline & Holiday & 0.0051 & 60.0 & $43.2 \pm 2.2 \%$ \\
\hline \multirow[t]{3}{*}{ Quest $^{\circledast}$} & Quest $^{\circledR} 3$ Nicotine free & 0.0091 & 51.9 & $50.2 \pm 1.3 \%$ \\
\hline & Quest $^{\circledR} 1$ Low Nicotine & 0.0087 & 53.4 & $46.5 \pm 0.3 \%$ \\
\hline & & Mean ( \pm SEM) & & $47.7 \pm 0.7 \%$ \\
\hline
\end{tabular}

Measured $\mathrm{IC}_{50}$ values for TPM samples were used to calculate the tar concentration at the $\mathrm{IC}_{50}$ and the predicted MAO-A inhibition at $0.01 \mathrm{mg} / \mathrm{mL}$ tar. This latter calculation is compared to the MAO-A inhibition at $0.01 \mathrm{mg} / \mathrm{mL}$ measured experimentally (\% of control). The mean inhibition of all brands is $47.7 \pm 0.7 \%$ ( $n=17$ brands tested in triplicate).

To test this theory, TPM samples were normalized to an arbitrary tar concentration, and then re-tested for MAO-A inhibition. This experiment also served to test if there was any 
qualitative difference in TPM composition with respect to MAO-A inhibition between TPM samples.

The $I C_{50}$ values derived for each TPM sample were used to predict the percentage MAO-A inhibition at a standard tar concentration $(0.01 \mathrm{mg} / \mathrm{mL})$, and these values were then confirmed experimentally by normalizing all samples to a tar concentration of $0.01 \mathrm{mg} / \mathrm{mL}$ and assaying for MAO-A inhibition (Table 11). Once again the inhibition values calculated from the $I C_{50}$ predicted the actual inhibition accurately; the difference between the predicted and measured inhibition values differing by a mean of $4.9 \%$. TPM samples were diluted to 0.01 $\mathrm{mg} / \mathrm{mL}$ tar, as earlier testing indicated this should inhibit the MAO-A enzyme preparation by approximately $50 \%$.

MAO-A inhibition following exposure to TPM samples normalised to $0.01 \mathrm{mg} / \mathrm{mL}$ tar

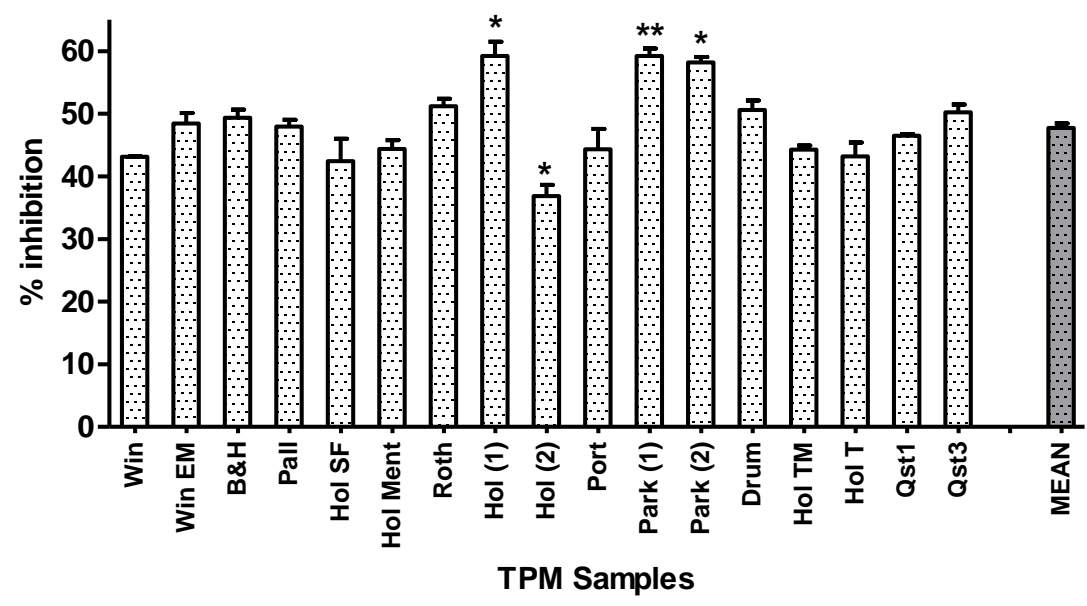

\begin{abstract}
Figure 24: TPM samples normalized to a tar concentration of $0.01 \mathrm{mg} / \mathrm{mL}$, showed significant differences in mean MAO-A inhibition (One-way ANOVA, $\mathrm{P}<0.0001$ ). In particular, the MAO-A inhibition induced by Holiday cigarette samples and Park Drive tobacco samples was significantly different from the mean of all samples (One-way ANOVA with Dunnett's multiple comparison post test; ${ }^{*} \mathrm{P}<0.01,{ }^{*} \mathrm{P}<0.001 ; \mathrm{n} \geq 3$ tests for each sample). Untreated control activity $=19.51 \pm 0.67 \mu \mathrm{g} / \mathrm{hr} / \mathrm{mg}$ protein. Sample abbreviations denote the cigarette and loose-leaf tobacco brands, and are defined in Appendix 9.2.
\end{abstract}

When the tar concentration was normalized to $0.01 \mathrm{mg} / \mathrm{mL}$ the mean MAO-A inhibition induced by different TPM extracts were found to be significantly different ( Fig. 24; one-way ANOVA, $\mathrm{P}<0.0001)$. This indicates there are significant differences in the ability of different TPM extracts to inhibit MAO-A when normalized to $0.01 \mathrm{mg} / \mathrm{mL}$ tar. In particular, the Park Drive tobacco samples (1) and (2) showed greater inhibition of MAO-A (59\% and 58\%, respectively) when compared to the mean MAO-A inhibition of all samples (48\%). The MAO-A 
inhibition induced by holiday regular cigarette samples was also significantly different from the mean, with sample (1) showing MAO-A inhibition significantly higher than the mean, and sample (2) showing inhibition significantly lower than the mean. These findings indicate there are significant qualitative differences in the composition of TPM between different tobacco products, and in the case of Holiday cigarettes, between different batches of the same tobacco product.

However, when the data were grouped by cigarette type, no significant difference was observed in MAO-A inhibition between TPM from manufactured cigarettes and TPM from loose-leaf tobacco. Loose-leaf tobacco TPM inhibited MAO-A activity by a mean of $49 \%$, and TPM from tailor-made cigarettes inhibited MAO-A by 47\% (Fig. 25). Therefore, while there are brand differences in the ability of TPM samples to inhibit MAO-A, there is no difference in MAO-A inhibition induced by manufactured cigarettes versus roll-your-own cigarettes when TPM concentration is normalized for tar content.

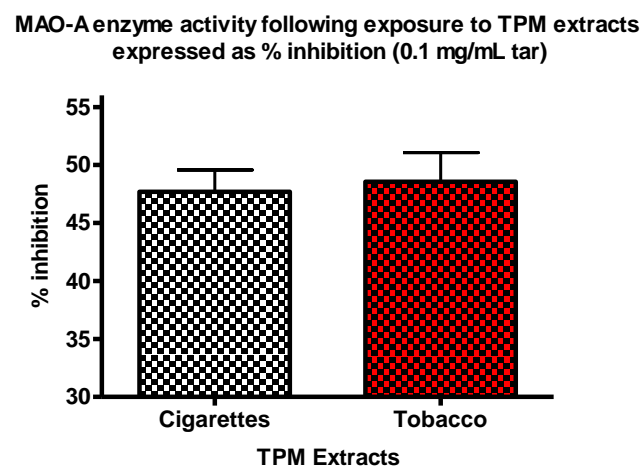

Figure 25: When TPM samples were normalized to a tar concentration of $0.01 \mathrm{mg} / \mathrm{mL}$, no significant difference was observed in the MAO-A inhibitory activity of TPM derived from roll-your-own tobacco products compared to that of factory-made cigarettes. Tobacco TPM inhibited MAO-A by a mean $48.6 \pm$ $2.5 \%(n=6)$ at $0.01 \mathrm{mg} / \mathrm{mL}$ tar, compared with cigarette TPM which inhibited MAO-A by a mean $46.7 \pm$ $1.5 \%(n=9)$.

\subsection{5 - RUYAN ${ }^{\circledR}$ Electronic Cigarette Exposures}

Samples taken from the RUYAN 0 and RUYAN 6 electronic cigarette cartridges were assessed for their potential inhibitory effects on MAO-A and MAO-B enzyme activity. According to the manufacturer, the solution used in the RUYAN cartridges contained known concentrations of nicotine, mixed with an unspecified tobacco extract. Neither the RUYAN 0 nor the RUYAN 6 sample had any effect on the activity of purified MAO-A or MAO-B enzymes (Fig. 26a, b). 
MAO-A (a) and MAO-B (b) reactions incubated with a concentration of RUYAN sample equivalent to $0.2 \mu \mathrm{M}$ nicotine for 15 mins showed no differences in enzyme activity compared to control activity. The activities of MAO-A and MAO-B were also measured in response to RUYAN sample concentrations equivalent to $2 \mu \mathrm{M}$ nicotine (Fig. 26a, b). Neither RUYAN 0 nor RUYAN 6 showed any inhibition of MAO-A or MAO-B at this higher concentration either. Thus, no difference in MAO-A or MAO-B activity was observed between untreated control enzyme, and enzyme treated with RUYAN samples at either $0.2 \mu \mathrm{M}$ or $2 \mu \mathrm{M}$ nicotine.

(a)

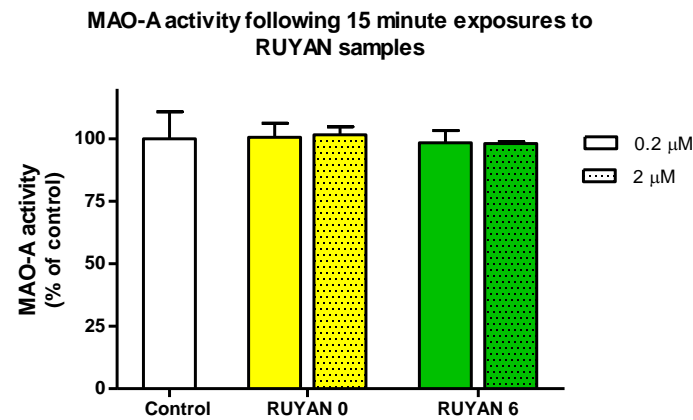

(b)

MAO-B activity following 15 minute exposures to RUYAN samples

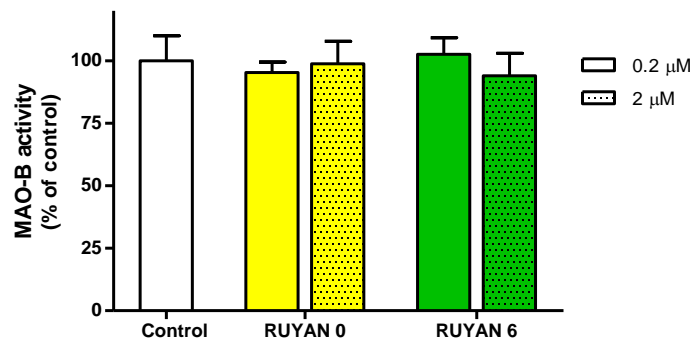

Figure 26: The effects of RUYAN 0 and RUYAN 6 samples were tested on purified recombinant MAO-A (a) and MAO-B (b) at concentrations corresponding to $0.2 \mu \mathrm{M}$ nicotine and $2 \mu \mathrm{M}$ nicotine for 15 mins. Neither treatment regimen resulted in any change in enzyme activity for MAO-A or MAO-B. (One-way ANOVA with Dunnett's post-test, $\alpha=0.05, \mathrm{n} \geq 3$ experiments for all conditions).

The effect of the RUYAN samples on MAO-A and MAO-B was also assessed following a longer incubation of 60 mins, at the same previously used concentrations. Neither RUYAN 0 nor RUYAN 6 elicited any change in MAO-A or MAO-B activity at either nicotine concentration after 60 mins (Fig. 27a, b). All RUYAN samples were assayed in parallel with TPM samples which showed inhibition of MAO-A and MAO-B, and served as a positive control.

In summary, the RUYAN electronic cigarette extracts were tested extensively using the modified kynuramine assay. Neither the RUYAN 0 nor RUYAN 6 samples caused any inhibition of MAO-A or MAO-B at concentrations of up to 10 -fold the nicotine concentration observed in the blood stream of heavy smokers. 
(a)

MAO-A activity following 60 minute exposure to RUYAN samples

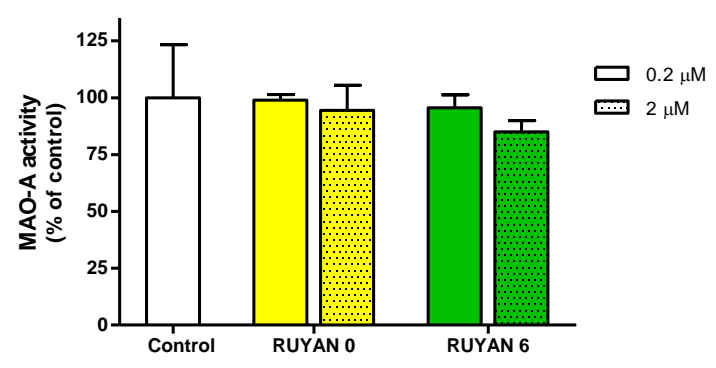

(b)

MAO-B activity following 60 minute exposure to RUYAN samples

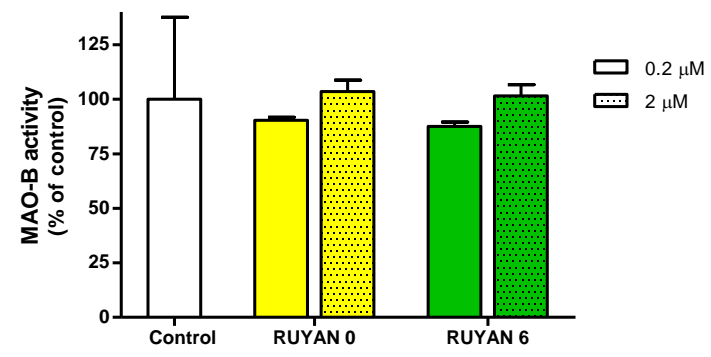

Figure 27: The effects of RUYAN 0 and RUYAN 6 samples were tested on purified recombinant MAO-A (a) and MAO-B (b) at concentrations corresponding to $0.2 \mu \mathrm{M}$ nicotine and $2 \mu \mathrm{M}$ nicotine for 60 mins. Neither treatment regimen resulted in any change in enzyme activity for MAO-A or MAO-B. (One-way ANOVA with Dunnett's post-test, $\alpha=0.05, \mathrm{n}=3$ experiments for all conditions).

\section{$\underline{4.4-\text { DISCUSSION }}$}

\subsection{1 - The Effects of Nicotine and TPM on Monoamine Oxidase Enzymes}

MAO enzymes were not inhibited by nicotine at concentrations of up to $10 \mu \mathrm{M}$. This is consistent with previously reported findings by Oreland et al.(1981) and Castagnoli et al. (2002) that nicotine alone does not act on MAO-A or MAO-B.

When purified MAO-A enzyme was treated with TPM extracts, a notable inhibition by both TPM from standard Holiday ${ }^{\circledR}$ brand cigarettes and denicotinized Quest ${ }^{\circledR}$ brand cigarettes was observed. MAO-A inhibition with standard cigarettes is consistent with previously reported evidence that MAO-A is inhibited by exposure to tobacco smoke, but the present study has confirmed that TPM derived from denicotinized tobacco smoke will also inhibit MAO-A to the same degree as standard tobacco. This comes as no surprise, given the evidence from this study that nicotine has no effect on MAO-A enzyme activity; therefore, the removal of nicotine from tobacco should not alter the inhibitory action of the TPM. However, Quest ${ }^{\circledR}$ cigarettes are not formulated from tobacco that has had the nicotine removed, but are made from tobacco strains which have been genetically modified to not express nicotine. Because it is uncertain what effect these genetic modifications may have on the composition of the tobacco, it is possible that TPM 
derived from these cigarettes may have significantly different MAO-A inhibitory activity to standard TPM. This was not the case, however, as Quest ${ }^{\circledR}$ TPM was found to inhibit MAO-A by the same degree as Holiday ${ }^{\circledR}$ TPM $(23 \%$, and $24 \%$, respectively), providing evidence that non-nicotine components of tobacco smoke are the cause of MAOA inhibition.

While Holiday ${ }^{\circledR}$ and Quest ${ }^{\circledR}$ TPM seemed to modestly reduce the activity of MAO-B compared to untreated enzyme at the concentrations tested, this difference was not statistically significant. The concentration-response analysis of MAO-B enzyme treated with TPM samples indicated that TPM caused inhibition of MAO-B, but the IC $C_{50}$ values for MAO-B inhibition were higher than those for MAO-A. This means higher concentrations of TPM were required to inhibit MAO-B than MAO-A, and may be due to lower concentrations of MAO-B inhibitors in the TPM samples, or less potent inhibition by the active compounds.

This finding is notable, as several previous studies have reported inhibition of MAO-B by standard tobacco extracts. Yu \& Boulton (1987) prepared a cigarette smoke solution by bubbling mainstream smoke through a phosphate buffer solution. They found that cigarette smoke solution significantly inhibited both MAO-A and MAO-B in rat mitochondrial preparations and rat lung tissue, noting that MAO-A showed greater inhibition than MAO-B. Using a similar method, Castagnoli et al. (2002) prepared tobacco smoke solutions by bubbling cigarette smoke through a phosphate buffer or hexane solution, and found that both solutions significantly inhibited MAO-B prepared from baboon liver mitochondria. Herraiz and Chaparro (2005) also reported that a cigarette smoke solution inhibited the enzyme activity of human recombinant MAO-A and MAO-B enzymes, and also found that MAO-A showed slightly more inhibition than MAO-B. These results are consistent with those of the present study.

None of the above studies made any attempt to quantify the concentration of the tobacco smoke solutions. It is likely that the tobacco smoke extracts used in those studies were much more concentrated than the solution used in the present study, leading to greater MAO-B inhibition, as the present study diluted the tobacco extract to a concentration that was physiologically relevant to human smokers. The tobacco smoke extract used in the present study was prepared from mainstream smoke particulates collected by filter through a smoking machine, while in each of the above experiments mainstream cigarette smoke was passed through a buffer solution to collect the tobacco 
smoke samples. It is also possible therefore that the collection method used by these studies may have captured some volatile or gaseous inhibitors of MAO-B that were not collected by the present method.

Although Holiday ${ }^{\circledR}$ and Quest $^{\circledR}$ TPM extract did not significantly inhibit recombinant MAO-B enzyme at TPM concentrations containing $0.2 \mu \mathrm{M}$ nicotine (or equivalent), the concentration-response analysis demonstrated that these extracts inhibited MAO-B at higher concentrations. Overall, the results of this study are in agreement with previously reported experiments (Herraiz \& Chaparro, 2005; P. H. Yu \& Boulton, 1987) that found tobacco smoke extract contained MAO-A and -B inhibitors, and was a more potent inhibitor of MAO-A than MAO-B.

\subsection{2 - Comparing Nicotine and Tar Yields Between Different Tobacco Products}

Because of the heterogeneous nature of tobacco, quantifying the TPM samples derived from tobacco samples proved difficult. Very little information was available on the composition or concentration of the TPM extracts, except for the mass of tar collected on the filter, the number of cigarettes collected on each filter, and the nicotine concentration of the TPM extracts, as measured by GC-MS. Because there was variation in the number of cigarettes per filter, and the manufacturers' reported nicotine yield per cigarette, it was difficult to ascertain the most appropriate way to measure TPM concentration. The nicotine concentration was used because it had been accurately measured by GC-MS. Tar concentrations for each TPM sample were also calculated from the measurements of tar mass collected on the filters.

TPM extracts from loose-leaf tobacco cigarettes had significantly higher tar:nicotine ratios than manufactured cigarettes, indicating loose-leaf tobacco TPMs generally had lower nicotine concentrations and higher tar concentrations than manufactured cigarette TPM samples. Thus, when TPM samples were normalized to contain $4 \mu \mathrm{M}$ nicotine, the loose-leaf tobacco samples contained a higher proportion of tar than the manufactured cigarettes. Similarly, when tar concentration was controlled for, TPM samples from loose-leaf tobacco cigarettes contained proportionally less nicotine than TPM samples from manufactured cigarettes. 
It should be noted that filter ventilation and cigarette paper porosity were not controlled for when preparing TPM samples for this study. Both of these factors have been found to be significant in affecting mainstream smoke yields (Stephens, 2007). The porosity and permeability of the cigarette wrap papers can increase ventilation while puffing, while chemical additives in the paper manufacturing process can increase cigarette burn rate, both of which reduce measured nicotine and tar yields. The cellulose acetate filters used in cigarettes to remove particulates from mainstream smoke, which also reduces nicotine and tar yields, and ventilated filters reduce these yields even further (Stephens, 2007). It is not known whether the cigarette wrap papers and filters used to roll the loose-leaf tobacco cigarettes have similar porosity and ventilation characteristics to those used in manufactured cigarettes; and it is also not known how similar the manufactured cigarette brands are to each other with respect to these factors.

When the tar concentration was normalized to $0.01 \mathrm{mg} / \mathrm{mL}$, the mean MAO-A inhibition induced by different TPM extracts was found to be significantly different (Fig. 24; one-way ANOVA, $\mathrm{P}<0.0001)$. This indicates there are significant differences in the ability of different TPM extracts to inhibit MAO-A when normalized to $0.01 \mathrm{mg} / \mathrm{mL}$ tar. In particular, the Park Drive tobacco samples (1) and (2) showed greater inhibition of MAO-A (59\% and 58\%, respectively) compared to the mean MAO-A inhibition of all samples (48\%). The MAO-A inhibition induced by Holiday regular cigarette samples was also significantly different from the mean, with sample (1) showing MAO-A inhibition significantly higher than the mean, and sample (2) showing inhibition significantly lower than the mean. These findings indicate there are significant qualitative differences in the composition of TPM between different tobacco products, and in the case of Holiday cigarettes, between different batches of the same tobacco product.

\subsection{3 - Comparing MAO Inhibition Between Different Tobacco Products}

A significant amount of variation was observed in the inhibitory potential of different brands of cigarettes and tobacco products (Fig. 24). This finding indicates that there are significant qualitative differences in the composition of TPM samples between different tobacco products, with respect to MAO inhibitors. TPM samples prepared from two different batches of Holiday brand cigarettes induced significantly greater, and lesser 
inihibition of MAO-A when compared to the mean of all samples. This demonstrates that there are also significant batch-to-batch differences in the MAO inhibitory potential of some cigarette brands. However, it should be noted that the data presented in Figure 24 are the mean MAO-A inhibition of individual TPM samples tested in triplicate. A more accurate and robust way to examine tobacco brand differences would be to prepare multiple independent TPM samples for each brand, however this option was not available to the present study due to the cost involved in generating the TPM filters.

Significant differences in the inhibition of MAO-A were also observed when comparing different types of tobacco product. When nicotine concentration was controlled, TPM samples from loose-leaf tobacco cigarettes showed significantly greater inhibition of MAO-A than manufactured cigarette TPMs. As discussed above, loose-leaf tobacco cigarettes were found to have a greater tar:nicotine ratio than manufactured cigarettes. This suggests that smokers of manufactured cigarettes may receive higher doses of nicotine than smokers of loose-leaf tobacco roll-your-own (RYO) cigarettes, and/or RYO smokers may receive higher doses of tar.

Studies on the puffing behavior of users of loose-leaf tobacco cigarettes, or RYO cigarettes, suggest that RYO smokers are likely to be exposed to higher tar concentrations. Laugesen et al. (2009) reported that although RYO smokers used less tobacco in their cigarettes (approx. $500 \mathrm{mg} /$ cigarette) than manufactured cigarettes (approx. $700 \mathrm{mg} /$ cigarette), they smoked more intensively by puffing more frequently and for longer periods than manufactured cigarette smokers, resulting in significantly greater exposure to tobacco smoke than manufactured cigarette smokers. An earlier study of RYO smokers found that although there was a wide variation in the weight of tobacco used per cigarette, the mean nicotine and tar yields per cigarette were similar to those of manufactured cigarettes (Darrall \& Figgins, 1998). Furthermore, a number of studies have shown that when smoking low-nicotine yield cigarettes, smokers will modify their puffing behavior by taking longer, deeper puffs to compensate for the reduced nicotine delivery (N. Benowitz et al., 2009; Scherer, 1999).

Taken together, these results suggest that smokers of loose-leaf tobacco smoke their RYO cigarettes more intensively to obtain similar nicotine yields to those found in manufactured cigarettes. In the present study, loose-leaf tobacco TPMs had a greater tar:nicotine ratio than manufactured cigarettes, indicating that when nicotine concentration is controlled, TPM from loose-leaf tobacco samples has a higher tar 
concentration than manufactured cigarettes, and thus a correspondingly higher concentration of MAO inhibitors. This explains why loose-leaf tobacco TPMs inhibited MAO-A more effectively than manufactured cigarette TPM samples (Fig. 23). When tar concentration was controlled for, no significant difference was observed in MAO-A inhibition between manufactured or loose-leaf tobacco cigarette TPMs (Fig. 25), which indicates that loose-leaf tobacco TPM caused greater MAO-A inhibition due to greater tar concentrations, and not due to higher inhibitor concentrations in the tar itself.

Therefore, users of loose-leaf tobacco cigarettes smoke are likely to be exposed to higher concentrations of tar and monoamine oxidase inhibitors than smokers of standard cigarettes. Exposure to higher concentrations of carcinogens and other harmful compounds known to be present in the tar fraction of cigarette smoke is also likely. Further studies into the smoking behavior of RYO cigarette smokers are warranted to confirm these findings. This could be tested by measuring levels of a number of biomarkers, such as cotinine (the major metabolite of nicotine) and 4(methylnitrosamino)-1-(3-pyridyl)-1-butanol (NNAL, a carcinogen and metabolite of tobacco-specific nitrosamine) (N. Benowitz et al., 2009) and by comparing levels of these compounds between smokers of loose-leaf tobacco and manufactured cigarettes. Additionally, it would be interesting to compare platelet MAO-B inhibition in loose-leaf tobacco smokers to manufactured cigarette smokers. To date, there has been little research into RYO cigarette smokers, but they represent a growing proportion of the smoking population in New Zealand (Laugesen et al., 2009).

\subsection{4-The RUYAN ${ }^{\circledR}$ Electronic Cigarette Does Not Inhibit MAO}

The cartridge fluid from the RUYAN E-cigarette was found to have no effect on the activity of either MAO-A or MAO-B. This is consistent with the cartridge fluid not being a tobacco-derived product. The RUYAN ingredients list contained a number of unspecified plant extracts (see Appendix 9.3) including vanilla extract, sweet fennel oil, geranium oil, and Kentucky tobacco extract. The composition of these plant extracts is unknown, and it is possible some may contain compounds that can inhibit MAO, particularly the tobacco extract. Since no MAO inhibition was measured, if there are inhibitors present in the cartridge formulation they are at sub-threshold concentrations. 
The present study tested the cartridge fluid of RUYAN E-cigarettes at concentrations corresponding to $0.2 \mu \mathrm{M}$ and $2 \mu \mathrm{M}$ nicotine. These concentrations were chosen because they represent the approximate physiological concentration of nicotine in the bloodstream of a smoker $(0.2 \mu \mathrm{M})$, and a ten-fold higher concentration ( $2 \mu \mathrm{M})$; however, it is not known whether these nicotine concentrations are relevant to people using the RUYAN E-cigarette. Because there is a paucity of published data on the E-cigarette, it is not known whether the E-cigarette shares the same pharmacokinetic profile as tobacco cigarettes; however, Bullen et al. (2010) found the pharmacokinetic profile of the RUYAN E-cigarette to be more similar to a nicotine inhalator than a tobacco cigarette. Their study reported that the bloodstream nicotine concentrations achieved by users of the $\mathrm{E}$ cigarette are considerably lower than those of regular cigarette smokers. A safety report on the RUYAN E-cigarette suggested that the device may deliver only a tenth of the nicotine that tobacco cigarettes do (Laugesen, 2008); however, an evaluation by the US Food \& Drug Administration found large variations in the amount of nicotine delivered by E-cigarettes (Westenberger, 2009).

The E-cigarette is not considered a legitimate smoking cessation device by the World Health Organization (WHO). Because there have been no reported rigorous, peerreviewed studies demonstrating that the E-cigarette is a safe and effective form of nicotine replacement therapy, WHO has stated that clinical studies and toxicity analyses are required before the organization can consider the device a smoking cessation aid (WHO, 2008). It is beyond the scope of this study to comment on the safety of the Ecigarette, or its efficacy as a smoking cessation aid, but the present study found that the RUYAN E-cigarette cartridge fluid had no effect on the activity of MAO-A or MAO-B enzymes at the concentrations tested. 


\section{Chapter Five: \\ MAO inhibition in cultured cells exposed to tobacco extracts}

\subsection{1 - SH-SY5Y Neuroblastoma as a Model of Human Neuronal Function}

The SH-SY5Y human neuroblastoma cell line has widely been used as an effective model of in vitro neuronal cell functioning and physiology. These cells display many of the same biochemical properties as human dopaminergic neurons in vivo, including the expression of functional muscarinic receptors, neuronal nAChRs, and $\mu$ and $\delta$ opioid receptors (2001; Vaughan et al., 1995). Since SH-SY5Y is an immortalized cell line, they are relatively easy to culture and care for, and cultures will continuously proliferate to provide large numbers of cells for different experiments (Constantinescu et al., 2007). This cell line has been used widely to provide biochemical insights into diverse neurobiological functions at a cellular level including mechanisms of addiction, neurotoxicity and apoptosis, and the etiology of neurodegenerative disorders like Alzheimer's and Parkinson's diseases.

Because SH-SY5Y cells are known to express catecholamine neurotransmitters (Pahlman et al., 1995; Vaughan et al., 1995), neuronal nAChRs (Ambrose et al., 2007; Lukas et al., 1993), and display MAO-A and MAO-B activity (Ekblom et al., 1996), they have been a popular in vitro model with which to study the effects of nicotine and tobacco on human neurons. A number of studies have already examined the response of SH-SY5Y cells to nicotine and tobacco extracts. Peng et al. (1997) demonstrated that chronic exposure to nicotine induced a significant up-regulation of nAChR subtypes, while Vaughan et al. (1993) demonstrated that nAChR agonists cause membrane depolarization and noradrenaline release in SH-SY5Y. Sokolova et al. (2005) used voltage clamp experiments to examine the desensitization of nAChR in SH-SY5Y cells due to nicotine. They reported that the nAChR on SH-SY5Y were readily desensitized by nicotine, but retained a small degree of electrophysiological responsiveness even to chronically administered nicotine concentrations. In our laboratory, Ambrose et al. (2007) measured the up-regulation of nAChR in SH-SY5Y cells in response to treatment with tobacco particulate matter (TPM) samples or nicotine alone. They reported a 
significant up-regulation of nAChRs in cells treated with both nicotine and TPM, but importantly, TPM was found to induce a greater up-regulation of these receptors than nicotine alone. This demonstrates that non-nicotine components of tobacco smoke are important up-regulators of nAChRs, and may influence tobacco dependence. A series of experiments by Dunckley \& Lukas $(2003,2006)$ investigated changes in gene expression in SHSY5Y cells using micro-array and qRT-PCR technology. They reported changes in the expression of 17 different genes with diverse cellular functions in SH-SY5Y cells after 1 hour exposure to nicotine and another 84 different genes after 24 hours exposure to nicotine. Their study confirmed that these changes in gene expression were dependent on the activation of $\mathrm{nAChRs}$, demonstrating that nicotine alters the expression of a diverse range of genes through binding to nAChRs.

While a number of investigators have used SH-SY5Y to examine cellular changes due to nicotine or tobacco exposure, no studies have yet investigated the effects of tobacco smoke compounds on the MAO activity of SH-SY5Y cells. To date, studies of these enzymes in SHSY5Y have largely focused on their role in the neuroprotective properties of clinical MAO inhibitors as treatments for Parkinson's and Alzheimer's diseases. However, using concentrations of ethanol relevant to human alcoholism, Ekblom et al. (1996) measured changes in MAO-A and MAO-B gene expression and enzyme activity in SH-SY5Y neuroblastoma and 1242 MG glioma cells. They reported a greater than $150 \%$ increase in MAO-B gene expression in 1242 MG glioma, and a more than $80 \%$ increase in MAO-B expression in SH-SY5Y treated for 24 hours with 100 mM ethanol. However, MAO-B enzyme activity was not altered by ethanol exposure in SH-SY5Y, and increased only slightly in 1242 MG cells. Neither gene expression nor enzyme activity of MAO-A was affected by ethanol exposure in 1242 MG or SH-SY5Y cells.

Because SH-SY5Y has been widely used to study the effects of nicotine on nAChRs, acetylcholine release, and gene expression, and has been demonstrated to express MAO enzymes, this cell line was chosen as in vitro model to examine the effects of tobacco smoke extracts on monoamine oxidase enzyme activity. 


\subsection{2- Glial Cells and Tobacco Addiction}

Glial cells and astrocytes play significant roles in a number of vital processes in the central nervous system, including synaptogenesis, regulation of neuronal function, and neuroplasticity (Lawrence et al., 2007). They assist in the regulation of neurotransmission and the metabolism of neurotransmitters, and are regarded as integral modulators of synaptic function (Sharma \& Vijayaraghavan, 2002).

There is growing evidence that astrocytes integrate neuronal activity in the central nervous system by detecting activity at the synapse, and propagating signaling to other neurons to modulate activity at remote synapses (for a review see Vijayaraghavan (2009)). Therefore, given the intimate role that glia and astrocytes play in mediating neuronal and synaptic activity, it is possible that changes in neuronal function due to the actions of drugs of abuse may also affect glial and astrocytic functioning, and vice versa.

Glial cells express a number of neurotransmitter transporters including the dopamine transporter (DAT) (Meng et al., 1999), serotonin transporter (SERT) (Inazu et al., 2001), norepinephrine transporter (NET) (Takeda et al., 2002), and the extra-neuronal monoamine transporter (EMT) (Inazu et al., 2003), as well as GABA and glutamate transporters (Schousboe et al., 2004). Studies have also shown that glia express high levels of monoamine oxidase activity in the brain (Levitt et al., 1982; Westlund et al., 1988) where they are believed to play a critical role in regulating synaptic neurotransmitter concentrations. Interestingly, glial cells also express a number of important receptor proteins, including the cannabinoid receptor CB1 (Stella, 2004), functional $\mu$ and $\delta$ opioid receptors (Watkins et al., 2005), and the nAChRs (Ono et al., 2008; Sharma \& Vijayaraghavan, 2002). Recent studies have suggested that glial functioning may be altered by exposure to drugs of abuse, which may subsequently lead to changes in neuronal function (Lawrence et al., 2007; Vijayaraghavan, 2009). At present, little is known about the effects of drug abuse on glial cells and glial-neuronal signalling, or what role these cells might play in the establishment or maintenance of addiction, but this is a rapidly emerging area of research (Vijayaraghavan, 2009).

Several studies have already examined the effects of nicotine and tobacco on glial function. For example, Knackstedt et al. (2009) reported that the glial glutamate transporter protein GLT-1 was down-regulated in the nucleus accumbens of rats self-administering nicotine. They reported that GLT-1 is also down-regulated in the nucleus accumbens after cocaine administration, and speculated that this protein may be relevant to the development and 
maintenance of drug dependence. Teaktong et al. (2003) reported a significant downregulation in the abundance of astrocytic $\alpha 7$ nAChRs in the brains of human smokers and exsmokers, compared to non-smokers. Another study by Mazzio et al. (2005) examined the effects of cigarette smoke condensates (CSC) on the activity of MAO and inducible nitric oxide synthase (iNOS) in rat C6 glioma cells to investigate the mechanisms responsible for tobacco's neuroprotective action against Parkinson's disease. They found that condensates from standard tobacco Marlboro cigarettes and 2R4F Kentucky reference cigarettes both significantly inhibited iNOS activity and total MAO activity. Both CSCs induced a mixed type inhibition of total MAO in glial cells, although the study noted that concentrations of CSCs that inhibited MAO in $\mathrm{C} 6$ glioma cells were toxic to N2A neuroblastoma. The viability of $\mathrm{C} 6$ cells exposed to these concentrations was not tested.

Thus, evidence reported to date suggests that glial cell function is altered by exposure to nicotine and tobacco smoke, and this effect may play a role in tobacco dependence. However, much more research into the effects of tobacco smoke on glial function and glialneuronal interactions is required to fully elucidate what role these cells might play in addiction mechanisms.

\section{$\underline{5.1 .3-\text { Objectives }}$}

The aim of the experiments in this chapter was to determine if tobacco extracts inhibit the activity of MAO enzymes in cultured neuronal and glial cells to the same degree as seen with isolated human recombinant enzymes (Chapter Four).

Cultured SH-SY5Y human neuroblastoma cells and U-118 MG human glioma cells were exposed to tobacco extracts derived from Holiday ${ }^{\circledR}$ brand cigarettes (HTPM) and Quest ${ }^{\circledR}$ denicotinized cigarettes (QTPM), as well as to nicotine alone. Holiday ${ }^{\circledR}$ brand cigarettes were chosen as representative of standard tobacco cigarettes available in New Zealand, as they were the highest volume selling cigarette in New Zealand at the commencement of the study (Fowles, 2003). Quest ${ }^{\circledast}$ cigarettes were chosen to investigate whether there was any difference in the MAO inhibition induced in cultured cells by denicotinized cigarettes compared to standard tobacco cigarettes. 
The SH-SY5Y cell line was chosen for this study to allow direct comparisons to be made with previously published experiments examining the response of SH-SY5Y to nicotine and tobacco extracts. The U-118 MG cell line was selected as the in vitro model of human glial cell function because it could be cultured under the same conditions as the SH-SY5Y cell line. Its glial origin is evidenced by its expression of glial fibrillary acidic protein (GFAP) (Feindt et al., 1995) - a glial and astrocytic cell marker.

SH-SY5Y cells and U-118 MG cells were exposed to tobacco extracts for up to five days to determine if total MAO activity was sensitive to acute and prolonged exposure to tobacco compounds. Based on the results reported in Chapter 4, it was expected that exposure to HTPM and QTPM would inhibit total MAO activity in both of these cells lines.

\section{2 - MATERIALS AND METHODS}

\subsection{1 - Exposure to Tobacco Particulate Matter}

The kynuramine assay, as described in Chapter 3 of this thesis, was used to measure total MAO enzyme activity in SH-SY5Y neuroblastoma cells and U-118 MG glioma cells following exposure to tobacco compounds. Cells were grown and cultured according to the methods described in section 2.1 .

\subsubsection{1 - Total MAO Activity in SH-SY5Y Cells}

Total MAO activity was measured in SH-SY5Y neuroblastoma cells treated with ethanol, nicotine, TPM from Holiday ${ }^{\circledR}$ brand cigarettes (HTPM), and TPM from Quest ${ }^{\circledR}$ denicotinized cigarettes (QTPM) for 1,3 , and 5 days continuously. SH-SY5Y cells were cultured in $600 \mathrm{~mL}$ culture flasks, and cells were allowed to adhere to the flask surface for at least a day before being treated. For each experiment, cells received nicotine or HTPM to a final concentration of $0.2 \mu \mathrm{M}$ nicotine, and equivalent volumes of ethanol or QTPM $(9.8 \mu \mathrm{L}$ per $30 \mathrm{~mL}$ media; $0.03 \%$ final concentration ethanol) were administered. Untreated SH-SY5Y cells served as a control. Extracts were added directly to the cell culture medium, and the cells were 
maintained in this medium for 1,3 , or 5 days without changing the medium (continuous treatment regimen; cont.). At the completion of the exposure regimen, the cells were harvested and lysates prepared according to the methods described in section 2.3.1 of this thesis.

Flasks of cells treated with a) ethanol, b) nicotine, C) HTPM and d) QTPMs were exposed for 1 day, 3 days, and 5 days without disruption before harvesting. Additionally, cells were also prepared for another treatment regimen (refreshed treatment regimen; ref.) which attempted to maintain more constant concentrations of test compounds in culture. Cultured cells received ethanol, nicotine, HTPM, and QTPM at the concentrations previously described and were incubated for 3 days continuously, at which time the medium in the flasks was removed by aspiration and replaced with fresh culture medium supplemented with new aliquots of ethanol, nicotine, HTPM, or QTPM. The cultures were then returned to the incubator for a further 2 days before being harvested and lysed.

\subsubsection{2 - Total MAO Activity in U-118 MG Cells}

Total MAO activity was also measured in U-118 MG glioma cells treated with ethanol, nicotine, HTPM, and QTPM for 5 days continuously. The U-118 cells were cultured according to the protocol described in section 2.1. Flasks of cells treated with a) ethanol, b) nicotine, c) HTPM, and d) QTPM were prepared, and incubated for 5 days continuously, without disruption (continuous treatment regimen). Nicotine and HTPM were added to a final concentration of $0.2 \mu \mathrm{M}$ nicotine, and ethanol and QTPM were added at equivalent volumes as described above. At the completion of the treatment period the cells were harvested and lysed as described in section 2.3.1.

\subsection{2 - Kynuramine MAO Activity Assay}

\subsubsection{1 - Kynuramine Microcentrifuge Tube Assay}

The kynuramine MAO activity assay was used to measure the total MAO enzyme activity in lysates prepared from SH-SY5Y and U-118 MG cells exposed to tobacco compounds using the microcentrifuge tube protocol described in section 2.4.2.1. Briefly, cell lysates were 
quantified with respect to total protein concentration using a BCA protein assay kit (Pierce. Rockford, IL, USA.) and diluted to working concentrations with sodium phosphate buffer (50 $\mathrm{mM}$ sodium phosphate, $150 \mathrm{mM}$ sodium chloride, $\mathrm{pH}$ 7.4). Cell lysates were added to $1.5 \mathrm{~mL}$ plastic microcentrifuge tubes in $100 \mu \mathrm{L}$ aliquots, and $870 \mu \mathrm{L}$ of sodium phosphate buffer was added. The microcentrifuge tubes were then incubated in a $37^{\circ} \mathrm{C}$ water bath for 5 mins to equilibrate the reaction temperatures. The assay reaction was started with the addition of a $30 \mu \mathrm{L}$ aliquot of kynuramine hydrobromide (Sigma-Aldrich, Inc.), diluted to a working concentration of $3.07 \mathrm{mM}$ unless otherwise stated. Reaction tubes were returned to the water bath and incubated at $37^{\circ} \mathrm{C}$ for 30 mins. The reaction was terminated by addition of $300 \mu \mathrm{L}$ of $0.4 \mathrm{M}$ perchloric acid (Sigma-Aldrich, Inc.) to each tube, and the tubes were then centrifuged at $13,000 \times \mathrm{g}$ for $30 \mathrm{sec}$ at $4^{\circ} \mathrm{C}$ to pellet any precipitated proteins.

Each reaction solution was aliquoted $(100 \mu \mathrm{L})$ into the wells of a black polystyrene 96 -well microwell plate (Greiner Bio-one) in triplicate. The 4-hydroxyquinoline (4-HQ) reference standard was diluted over the range $0-5000 \mathrm{nM}$, and $100 \mu \mathrm{L}$ aliquots of each standard were added in triplicate to different wells of the microwell plate to construct a standard curve. $\mathrm{NaOH}$ ( $200 \mu \mathrm{L}$ of a $1 \mathrm{M}$ solution) was added to each well to induce fluorescence of the 4-HQ product, and the fluorescence was measured immediately at $318 \mathrm{~nm}$ excitation and $380 \mathrm{~nm}$ emission wavelengths with a SpectraMax Gemini spectrophotometer. A standard curve was plotted from the 4-HQ reference standard fluorescence data, and this was used to quantitate the concentration of product formed for each sample.

\subsubsection{2 - Lineweaver-Burk Analysis}

SH-SY5Y cell lysates from cells treated with tobacco compounds for 3 or 5 days were used to measure total MAO activity in response to varied kynuramine concentrations. The data from these experiments was used to derive a Lineweaver-Burk plot to examine the nature of MAO inhibition induced by the TPM samples. Two samples each of cells treated with a) ethanol, $b$ ) nicotine, c) HTPM, and d) QTPM for three days, and two samples each of cells treated for five days continuously were used. Each sample was assayed in triplicate using kynuramine concentrations of $0.25 \mu \mathrm{M}, 0.75 \mu \mathrm{M}, 3.07 \mu \mathrm{M}$, and $7.50 \mu \mathrm{M}$.

Double reciprocal data were derived, and GraphPad Prism v5.0 (Graphpad software, Inc. San Diego, CA, USA) was used to plot Lineweaver-Burk plots for SH-SY5Y cells treated with tobacco 
compounds and analyse the data by non-linear regression modelling. The $\mathrm{V}_{\max }$ and $\mathrm{K}_{\mathrm{m}}$ of the reactions were calculated to determine the nature of TPM inhibition of MAO.

\section{$\underline{5.3-\text { RESULTS }}$}

\subsection{1 - Effects of Ethanol Exposure on Total MAO Activity in SH-SY5Y Cells}

Total MAO activity was measured for SH-SY5Y cell lysates treated with $0.03 \%$ ethanol for 1,3 , and 5 days continuously, and for 5 days under the refreshed medium regimen, and compared to the MAO activity measured in untreated SH-SY5Y cell lysates (Fig. 28). Ethanol exposure $(5.6 \mu \mathrm{M})$ did not significantly affect total MAO activity in SH-SY5Y cells at any of the time points tested.

Total MAO activity in SH-SY5Y cells treated with

ethanol vehicle.

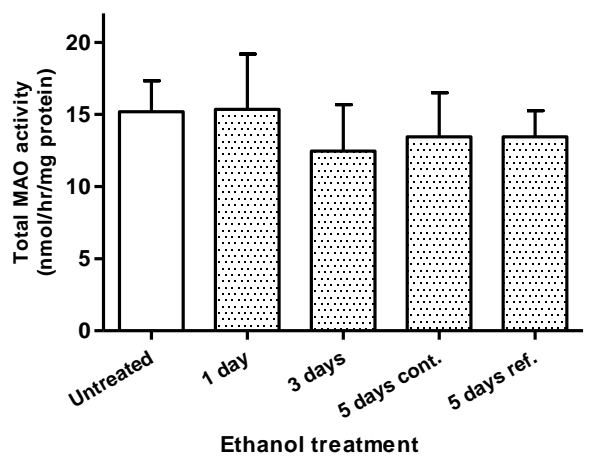

Figure 28: Total MAO enzyme activity in cultured SH-SY5Y cells treated with ethanol for 1, 3, and 5 days continuously (cont.), and 5 days under the refreshed medium regimen (ref.). Ethanol exposure induced no statistically significant change in total MAO enzyme activity at any time point (One-way ANOVA with Dunnett's multiple comparison post test; $n \geq 4$ independently prepared flasks for all conditions).

\subsection{2 - MAO Inhibition in SH-SY5Y Cells Treated with Tobacco Extracts}

Lysates prepared from SH-SY5Y cells treated for 3 or 5 days with ethanol, nicotine, HTPM, or QTPM were assayed for total MAO activity over a range of kynuramine substrate concentrations. Total MAO activity in lysates treated with HTPM and QTPM for three days 
was not significantly different from the MAO enzyme activity measured in the ethanol-treated control (Fig. 29a). In lysates prepared from cells treated for 5 days, nicotine, HTPM, and QTPM all inhibited total MAO activity in these cells (Fig. 29b).

Double reciprocal data from the 5-day treatment experiments were used to construct several Lineweaver-Burk plots to examine the nature of the inhibition induced by nicotine, HTPM, and QTPM (Fig. 30a).

(a)

Total MAO enzyme activity Vs. substrate concentration in SH-SY5Y cells treated with tobacco extracts for 3 days .

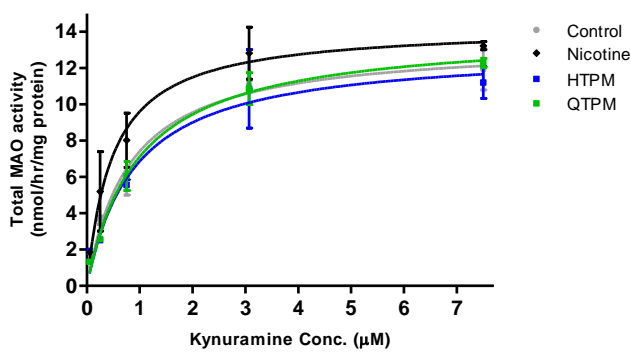

(b)

Total MAO activity Vs. substrate concentration in SH-SY5Y cells treated with tobacco extracts for 5 days.

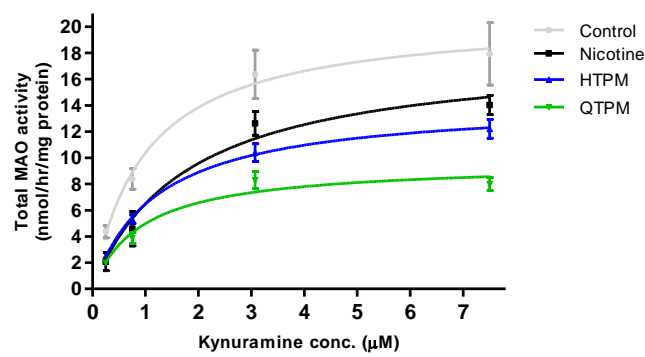

Figure 29: Cultured SH-SY5Y cells treated for (a) 3 or (b) 5 days continuously with nicotine, HTPM, or QTPM, and tested with the modified kynuramine assay over a range of substrate concentrations. Control cells received treatment with $0.03 \%$ ethanol vehicle. Each data point represents the mean and SEM of 2 reactions assayed in triplicate from 2 independent reactions $(n=6)$.

Treatment with nicotine appeared to increase the $\mathrm{K}_{\mathrm{m}}$ for total MAO activity, but $\mathrm{V}_{\max }$ was not greatly changed from ethanol-treated controls (Fig. 30b). This suggests nicotine induced a competitive inhibition in SH-SY5Y lysates. Treatment with HTPM (Fig. 30c) reduced the $\mathrm{V}_{\max }$ but increased $\mathrm{K}_{\mathrm{m}}$, suggesting a mixed type inhibition, but QTPM (Fig. 30d) altered only $\mathrm{V}_{\max }$, indicating a non-competitive enzyme inhibition. Linear regression analysis of the lines produced by the Lineweaver-Burk analysis found that the difference between the slopes of each line were very significant (GraphPad Prism v5.0), but changes in $V_{\max }$ and $K_{m}$ between treatments were not found to be statistically significant (Student's two-tailed t-test). 
(a)

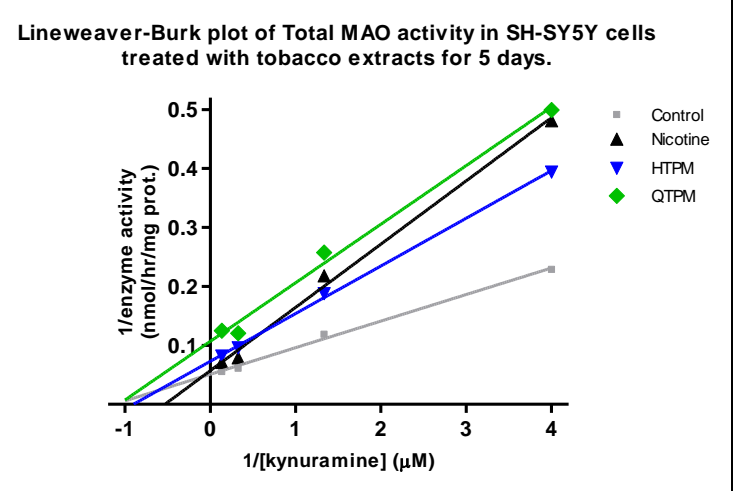

(b) Lineweaver-Burk plot of total M AO activity in SH-SY5Y cells

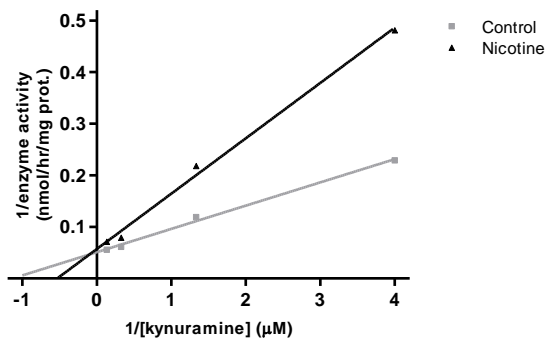

(c)

Lineweaver-Burk plot of total MAO activity in SH-SY5Y cells treated with HTPM for 5 days.

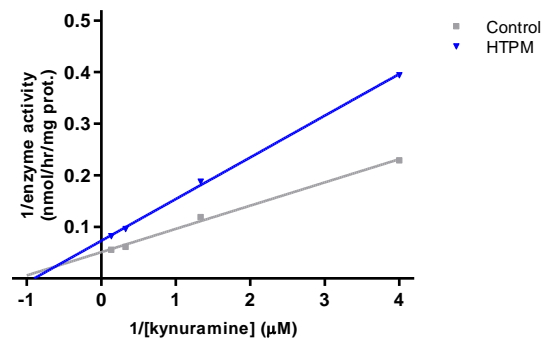

(d)

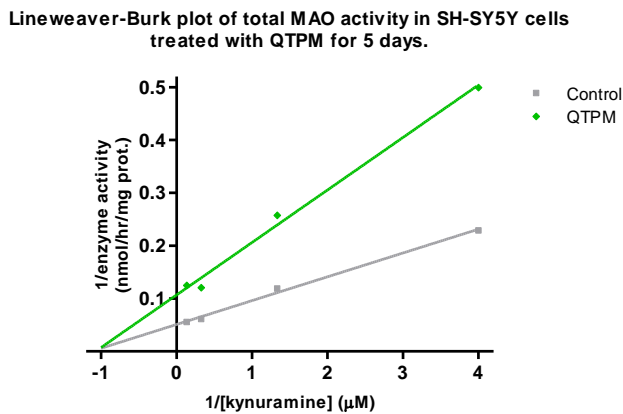

Figure 30: (a) Lineweaver-Burk plot for the data presented in Figure 29b. (b) Five days of nicotine treatment resulted in competitive inhibition of total MAO, while exposure to HTPM induced a mixed type inhibition (c), and exposure to QTPM non-competitively inhibited total MAO in SH-SY5Y cells (d). Control cells received treatment with $0.03 \%$ ethanol vehicle. Each data point represents the mean and SEM of 2 reactions assayed in triplicate in 2 independent experiments $(n=6)$. 
Table 12: Enzyme reaction parameters for treated SH-SY5Y cells

\begin{tabular}{|c|c|c|c|}
\hline 5 Day Treatment & $\begin{array}{c}V_{\max } \\
\text { (nmol/hr/mg protein) }\end{array}$ & $\begin{array}{l}K_{m} \\
(\mu M)\end{array}$ & Inhibition \\
\hline Ethanol control & $20.78 \pm 2.8$ & $1.002 \pm 0.07$ & \\
\hline Nicotine & $18.12 \pm 2.6$ & $1.784 \pm 0.66$ & Competitive \\
\hline HTPM & $14.27 \pm 0.4$ & $1.209 \pm 0.06$ & Mixed \\
\hline QTPM & $9.63 \pm 0.6$ & $0.933 \pm 0.06$ & Non-competitive \\
\hline
\end{tabular}

\subsection{3 - Tobacco Extract Exposure and Total MAO Activity in SH-SY5Y Cells Over Time}

Total MAO enzyme activity was also measured in SH-SY5Y cell lysates treated with nicotine, HTPM, and QTPM for 1, 3, or 5 days continuously, or for 5 days under the refreshed medium regimen.

In SH-SY5Y cells treated with HTPM and QTPM extracts for 24 hours, total MAO activity was inhibited significantly compared to ethanol-treated controls (Fig. 31). Cells treated with HTPM and QTPM inhibited total MAO activity by $38 \%$ and $37 \%$, respectively. Thus, there was no difference in MAO inhibition between HTPM or QTPM treatments.

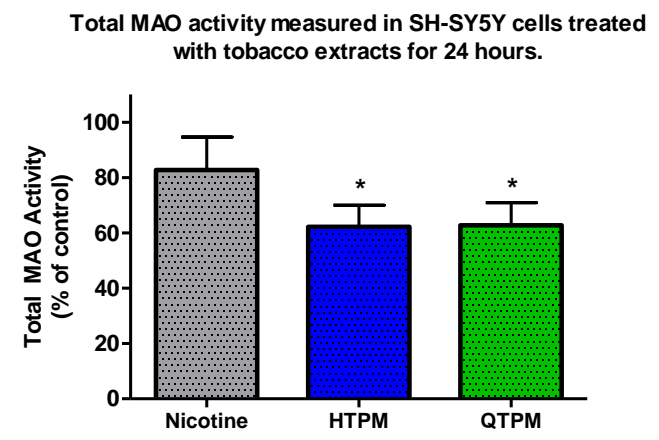

Figure 31: Changes in total MAO enzyme activity in cultured SH-SY5Y cells treated with tobacco constituents for 24 hours. Exposure to $0.2 \mu \mathrm{M}$ nicotine elicited no change in total MAO enzyme activity relative to ethanol-treated control cultures; while, treatment with HTPM and QTPM significantly inhibited MAO activity to $62 \%$ and $63 \%$ of control activity, respectively (One-way ANOVA with Dunnett's multiple comparison post-test; $\mathrm{n} \geq 6$ flasks for all conditions, from 4 independent preparations. $\left.{ }^{*} \mathrm{P}<0.05\right)$ (Control activity $=9.8 \pm 1.3 \mathrm{nmol} / \mathrm{hr} / \mathrm{mg}$ protein). 
Unexpectedly, no change in total MAO activity was observed in SH-SY5Y cells treated for 3 days with nicotine, HTPM, or QTPM (Fig. 32). This experiment measured MAO activity in cell lysates treated with ethanol, nicotine, HTPM, and QTPM, but no significant differences were observed in total MAO activity in these exposure groups.

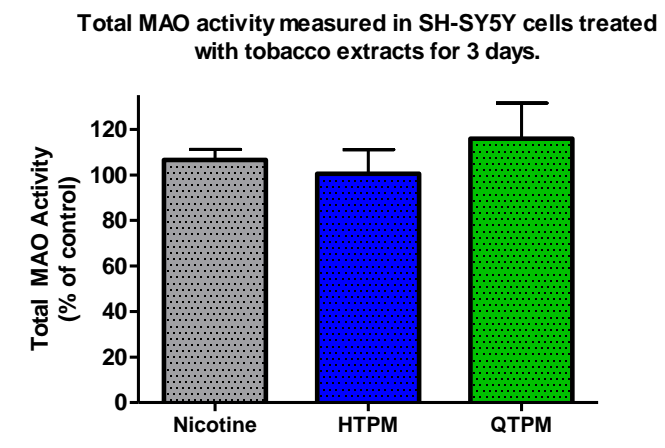

Figure 32: Changes in total MAO enzyme activity in cultured SH-SY5Y cells treated with tobacco compounds for 3 days. No statistically significant differences were recorded in total MAO enzyme activity in cell cultures treated with nicotine, HTPM, or QTPM for 3 days continuously (One-way ANOVA with Dunnett's multiple comparison post-test; $\mathrm{n} \geq 8$ flasks for all conditions, from 6 independent preparations). (Control activity $=8.3 \pm 1.2 \mathrm{nmol} / \mathrm{hr} / \mathrm{mg}$ protein).

After 5 days of exposure to HTPM and QTPM, significant inhibition of total MAO enzyme activity was seen in SH-SY5Y cells, similar to that observed after 1 day of treatment with these compounds (Fig. 33). Cell cultures treated with HTPM were inhibited by $18 \%$, and those treated with QTPM were inhibited by $22 \%$ compared to ethanol-treated controls. Interestingly, cells treated with nicotine alone also showed significant inhibition of MAO activity (15\% inhibition). This was unexpected as no inhibition of MAO was recorded in cells treated with purified nicotine for 1 or 3 days (Fig. 34).

In summary, after 1 day of exposure only cells treated with HTPM or QTPM samples showed statistically significant inhibition of MAO activity relative to control; however, after 3 days treatment no MAO inhibition was observed in any treatment group. Following exposure to the test compounds for 5 days continuously, cells treated with nicotine, HTPM, and QTPM all demonstrated statistically significant inhibition of total MAO activity (Fig. 34). 


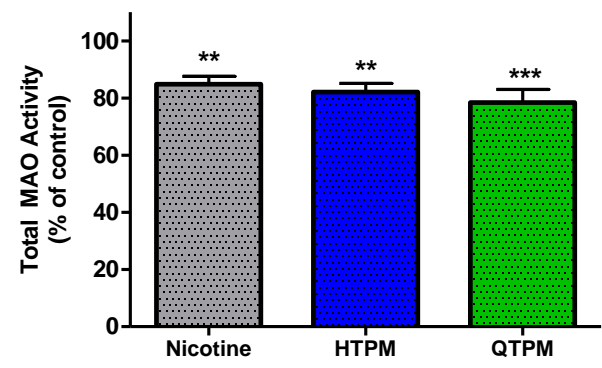

Figure 33: Inhibition of total MAO enzyme activity in cultured SH-SY5Y cells treated with tobacco compounds for 5 days continuously. Exposure to $0.2 \mu \mathrm{M}$ nicotine inhibited total MAO activity to $85 \%$ of control activity; whereas, HTPM and QTPM inhibited MAO activity to $82 \%$ and $78 \%$ of control, respectively. This inhibition was found to be statistically significant (One-way ANOVA with Bonferroni's multiple comparison post-test; $\mathrm{n} \geq 12$ flasks for all conditions, from 10 independent preparations. ** $\mathrm{P}<0.01, * * * \mathrm{P}<0.001)$. (Control activity $=14.4 \pm 1.7 \mathrm{nmol} / \mathrm{hr} / \mathrm{mg}$ protein).

Summary of changes in total MAO activity over a 5-day treatment period.

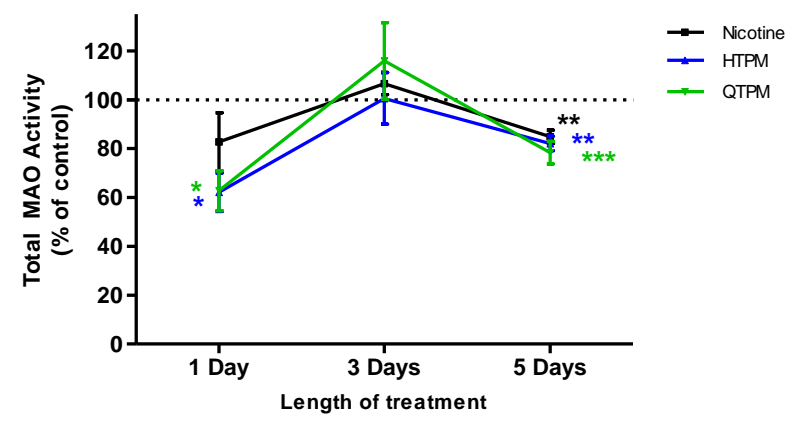

Figure 34: Differences in total MAO enzyme activity inhibition in cultured SH-SY5Y cells treated with various tobacco constituents for up to 5 days. This figure combines the data presented in figures 31,32 , and 33 ( $\left.{ }^{*} \mathrm{P}<0.05,{ }^{* *} \mathrm{P}<0.01,{ }^{* * *} \mathrm{P}<0.001\right)$.

When cultured SH-SY5Y cells were treated with tobacco compounds for 5 days using the refreshed medium regimen, total MAO activity was unexpectedly increased relative to ethanol-treated controls (Fig. 35). This was the first indication that TPM extracts could stimulate rather than inhibit MAO activity. Nicotine treatment increased total MAO activity to $185 \%$, HTPM treatment to $220 \%$, and QTPM treatment to $177 \%$ of control activity. Ethanoltreated controls that also received refreshed medium and treatment showed no change in total MAO activity relative to other treatment regimens (Fig. 28). Due to the small sample size and the variation present in the data, only the increase in MAO activity due to HTPM 
treatment was found to be statistically significant when tested by one-way ANOVA and Bonferroni's post-test.

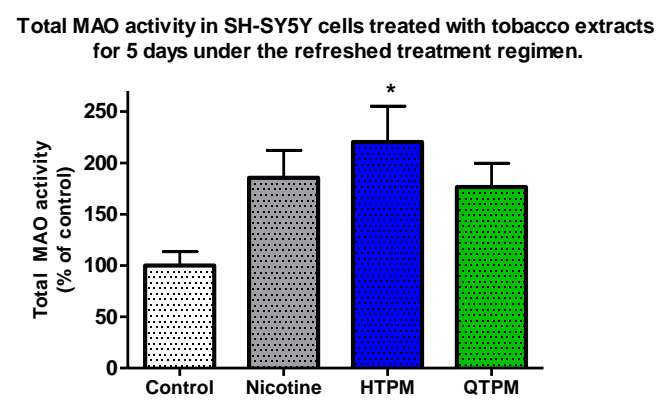

Figure 35: Changes in total MAO enzyme activity in cultured SH-SY5Y cells treated with tobacco extracts for 5 days using the refreshed medium regimen. Overall, total MAO activity increased in cells treated with nicotine, HTPM, and QTPM, although this change was only found to be statistically significant in the case of HTPM-treated cells. Total MAO activity increased to $220 \%$ of ethanol-treated controls in cells cultured with HTPM under the refreshed medium regimen. These data were tested by one-way ANOVA with Bonferroni's multiple comparison post-test; $\mathrm{n} \geq 4$ flasks for all conditions; ${ }^{*} \mathrm{P}<0.05$. (Control activity $=13.4 \pm 1.8 \mathrm{nmol} / \mathrm{hr} / \mathrm{mg}$ protein).

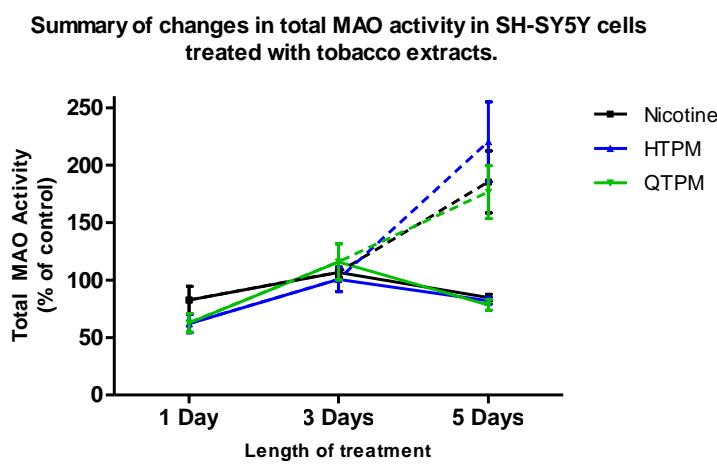

Figure 36: Summary of differences in inhibition of total MAO enzyme activity in cultured SH-SY5Y cells treated with tobacco extracts for up to 5 days, using either continuous (solid lines) or refreshed medium (dashed lines) treatment regimens. This figure combines the data presented in figures 34 and 35 . The results show an increase in total MAO activity in response to nicotine, HTPM and QTPM in cells cultured with the refreshed medium regimen, compared to the 5-day continuous treatment.

To summarize, nicotine, HTPM, and QTPM induced significant inhibition of total MAO activity in SH-SY5Y cells treated for 5 days undisturbed, but these compounds appeared to increase total MAO activity when cells were treated with the refreshed medium regimen (Fig. 36). The increase in MAO activity following the refreshed medium regimen was only significant for cells treated with HTPM, in part because of the small sample size $(n=4)$. 


\subsection{4 - Effects of Tobacco Extract Exposure on Total MAO Activity in U-118 MG Cells}

Total MAO activity was measured in U-118 MG cells treated with ethanol, nicotine, HTPM, and QTPM for 5 days continuously. MAO activity in untreated U-118 cells was found to be considerably lower than the MAO activity measured in SH-SY5Y cells. Untreated U-118 cells had a mean activity of $0.94( \pm 0.1) \mathrm{nmol} / \mathrm{hr} / \mathrm{mg}$ protein, compared to the mean MAO activity in SY5Y cells of $15.2( \pm 2.1) \mathrm{nmol} / \mathrm{hr} / \mathrm{mg}$ protein.

Treatment with the ethanol vehicle for 5 days continuously appeared to decrease total MAO activity, but this change was not significant (Fig. 37a). MAO activity measured in U-118 MG cells treated with nicotine, HTPM, and QTPM was compared to the activity measured in the ethanol control. Although treatment with nicotine, HTPM, and QTPM all seemed to increase total MAO activity in U-118 MG cells, there was a high degree of variation in the data, and no statistically significant changes were observed (Fig. 37b).

(a)

Total MAO activity measured in U118 MG cells treated with tobacco components for 5 days.

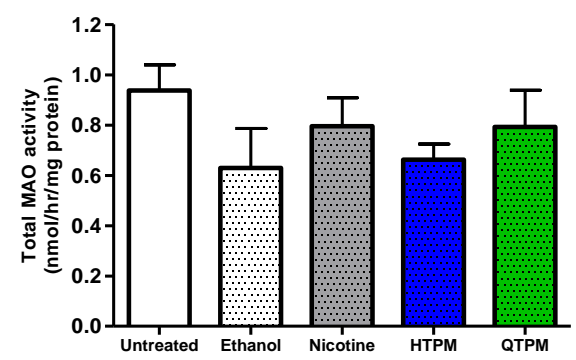

(b)

Total MAO activity expressed as percent of control in U-118 MG cells treated with tobacco components for 5 days.

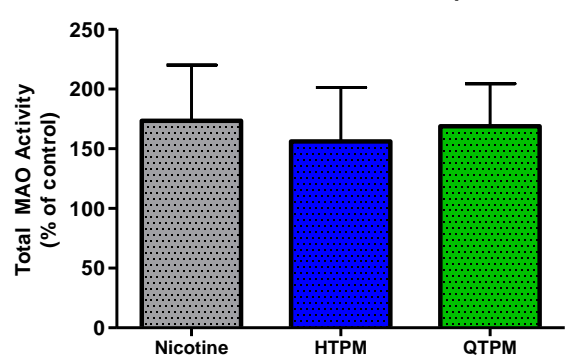

Figure 37: Total MAO enzyme activity in cultured U-118 MG cells treated with tobacco compounds for 5 days continuously, expressed as (a) total MAO activity ( $\mathrm{nmol} / \mathrm{hr} / \mathrm{mg}$ protein) and (b) as a percentage of the activity measured in ethanol-treated controls. No significant changes in MAO activity were measured in cells exposed to nicotine, HTPM, or QTPM compared to ethanol-treated controls (One-way ANOVA with Bonferroni's multiple comparison post-test; $n=7$ flasks for all conditions, from 4 independent preparations). 


\section{$5.4-$ DISCUSSION}

\subsection{1 - Effects of Ethanol Exposure on Total MAO Activity in SH-SY5Y Cells}

Ethanol had no effect on total MAO enzyme activity in SH-SY5Y cells under any of the treatment regimens. This confirmed that the ethanol vehicle used to solubilize the TPM samples did not contribute to inhibition of MAO in this cell line. These results a re in agreement with Ekblom et al. (1996) who also reported no influence of ethanol on the activity of MAO, although that study exposed SH-SY5Y to much higher ethanol concentrations ( $100 \mathrm{mM}$, compared to $5.6 \mathrm{mM}$ in the present study). Some variation was seen in the measures of total MAO activity, but these were comparable to the variation in activity seen in untreated cells (Fig. 28). This variation is thought to represent natural biological variation in MAO activity between cultures of SH-SY5Y cells, and variation inherent in the assay.

\subsection{2 - Standard and De-Nicotinized Tobacco Extracts Inhibit MAO in SH-SY5Y}

SH-SY5Y human neuroblastoma cells exposed to HTPM and denicotinized QTPM tobacco smoke extracts for 5 days continuously showed significant inhibition of total MAO. Standard tobacco smoke extract has previously been reported to inhibit MAO activity (Castagnoli et al., 2002; Mazzio et al., 2005; P. H. Yu \& Boulton, 1987), but the present study is the first to demonstrate that denicotinized tobacco extract inhibits MAO to the same degree as standard tobacco. This finding further confirms the theory that nonnicotine components of mainstream tobacco smoke are responsible for MAO inhibition. This result also suggests that smokers of denicotinized Quest ${ }^{\circledR}$ cigarettes are likely to have reduced levels of brain and peripheral MAO enzyme activity, similar to smokers of standard tobacco cigarettes.

Lineweaver-Burk and non-linear regression analyses were used to examine the type of inhibition induced by nicotine and tobacco extracts in SH-SY5Y cells treated for 5 days. HTPM appeared to induce a mixed-type inhibition, which is consistent with previous published studies. Yu and Boulton (1987) and Mazzio et al. (2005) both reported that cigarette smoke extracts induced a mixed-type inhibition of rat lung MAO and total MAO in C6 glioma, respectively. Herraiz and Chaparro (2005) examined inhibition of purified MAO-A and $-B$ enzymes, and found cigarette smoke extracts competitively inhibited 
MAO-A, but inhibited MAO-B by a mixed-type inhibition. The present study was unable to distinguish between MAO-A and MAO-B activity in the SH-SY5Y lysates, but the results obtained are still consistent with these previous studies.

Denicotinized QTPM also inhibited total MAO, but the type of inhibition was noncompetitive. This finding was unexpected. QTPM was presumed to be similar in composition to HTPM but without the nicotine content, and thus, was expected to contain the same MAO inhibitors as standard tobacco. The finding that QTPM induces a different type of MAO inhibition than HTPM could suggest that some MAO inhibitors present in HTPM are absent in QTPM. This would suggest that although QTPM inhibits total MAO to the same degree as HTPM in cultured cells, a different cohort of MAO inhibitors may be responsible for the inhibition, accounting for the different type of inhibition observed. There is some evidence to support this theory, as a study examining the chemical composition of TPM from Quest nicotine-free cigarettes reported Quest cigarettes contained significantly greater concentrations of 2-naphthylamine than the 2R4F reference cigarettes (J. Chen et al., 2008). 2-Naphthylamine is a carcinogen found in high concentrations in cigarette smoke, and is known to inhibit MAO-A and MAO-B by a mixed-type inhibition (Hauptmann \& Shih, 2001).

Differences in the inhibition data for HTPM and QTPM could also be due to the difficulty in interpreting the kinetic enzyme data for total MAO activity. Studies of enzyme inhibition kinetics are usually performed by exposing a single enzyme of a known concentration to a range of substrate concentrations in the presence or absence of a single inhibitor. The present study used whole cell homogenates that contained both MAO-A and $-B$ enzymes, and cells were treated with TPM samples that are known to contain a number of different MAO inhibitors. Total protein concentration was used to quantify the cell homogenate, and assumes that the concentration of MAO enzyme within the homogenates is equal between samples. However, it is possible that exposure to HTPM or QTPM may have down-regulated MAO in SH-SY5Y cells, and these lysates may not have the same concentration of MAO enzyme as untreated cells. Therefore, the Lineweaver-Burk analysis performed in the present study represents the inhibition of two enzymes (MAO-A and MAO-B) by multiple different inhibitors in TPM. The present experiment is similar to that described by Mazzio et al. (2005), in which whole C6 rat glioma cells were exposed to cigarette smoke condensates, and both competitive and non-competitive inhibition was measured in these cells. 
In the present study, exposure to purified nicotine also inhibited total MAO activity compared to control cells. This finding was unexpected, since previous studies have reported that nicotine has no direct effect on the activity of MAO enzymes (Castagnoli et al., 2002; Oreland et al., 1981). Kinetic analysis showed nicotine induced a competitive inhibition of total MAO in SH-SY5Y cells, but the present study also examined the effects of nicotine on purified MAO-A and MAO-B enzymes, and found that nicotine induced no inhibition in these preparations (section 4.3.1). Nicotine is known to cause increased release of dopamine, serotonin, and norepinephrine in vitro, and in the brain (Balfour \& Fagerstrom, 1996; N. L. Benowitz, 2008; Di Chiara \& Imperato, 1988; Pontieri et al., 1996; Rao et al., 2003; Vaughan et al., 1995; Wonnacott et al., 2005), and these catecholamines are natural substrates for MAO-A and MAO-B enzymes. It is possible that exposure to nicotine has increased concentrations of these neurotransmitters in the prepared cell lysates, and these natural substrates for MAO-A and MAO-B may compete with the kynuramine substrate used in this assay. Therefore, the decrease in 4-HQ fluorescent product may not reflect MAO-A or MAO-B inhibition, but that the native substrates for MAO-A and MAO-B are competing with the kynuramine assay substrate.

If this is the case, it could be argued that some of the inhibition induced by HTPM and QTPM after 5 days treatment was also due to kynuramine competition with native substrates. However, Lineweaver-Burk analysis of the inhibition of total MAO by HTPM and QTPM shows the inhibition type to be mixed, ie: competitive and non-competitive. This is in agreement with earlier studies that have reported a mixed-type of inhibition induced by tobacco compounds, as discussed above. Furthermore, the QTPM sample contains no nicotine, and therefore the decreased 4-HQ product formation observed in SH-SY5Y cells treated with QTPM must be due to inhibition of MAO, unless non-nicotine compounds in QTPM are also stimulating increased neurotransmitter release.

\subsection{3 - Changes in Total MAO Activity in SH-SY5Y Cells Exposed to Tobacco Compounds}

As expected, short-term one-day exposures to both HTPM and QTPM inhibited the total MAO activity of SH-SY5Y cells in culture. This is consistent with other studies that have reported inhibition of MAO-A and MAO-B after short-term exposures to tobacco smoke extracts (Castagnoli et al., 2002; Mazzio et al., 2005; P. H. Yu \& Boulton, 1987). 
Furthermore, this study has confirmed that tobacco smoke extracts inhibit MAO enzymes at concentrations relevant to human tobacco consumption. Previous studies have used much higher concentrations of tobacco smoke extract, or made no attempt to quantify the compounds in the extract. In the present study SH-SY5Y cells were exposed to a concentration of HTPM that contained $0.2 \mu \mathrm{M}$ nicotine. This concentration is very similar to the concentrations of nicotine attained in the blood stream of heavily dependent human smokers (S. G. Gourlay \& N. L. Benowitz, 1997; J.E. Rose et al., 1999). This study confirmed that MAO is inhibited by short-term exposure to tobacco smoke compounds at concentrations attained by human smoking behavior. This is also true of the denicotinized QTPM sample, which inhibited total MAO to the same degree as HTPM after a one-day exposure period.

MAO activity results after longer exposure periods are more difficult to explain. While HTPM and QTPM inhibited MAO activity after a one-day exposure, no inhibition was recorded in cells treated for three days continuously. This may be a result of natural variation in MAO activity from culture to culture, as earlier experiments demonstrated there was a high degree of variation in the levels of MAO activity in SH-SY5Y cells (section 3.3.2.5). It is also possible that after three days incubation with TPM the cells had metabolized or inactivated the MAO inhibitors in the TPM samples, allowing MAO activity to return to baseline. However, after five days continuous treatment, MAO activity was significantly inhibited in cells treated with HTPM, QTPM, and nicotine. As discussed above (section 5.3.2), inhibition by nicotine is potentially an artifact of the kynuramine substrate competing with natural MAO substrates, while inhibition due to TPM samples is presumed to be genuine. The present study did not test to determine if concentrations of ethanol and TPM compounds remained stable over the treatment period. A study by D'Addario et al. (2008) measured ethanol treatment concentrations in SH-SY5Y cell cultures over time and reported that ethanol concentrations decreased by $23 \%$ after 72 hrs in cells treated with $10 \mathrm{mM}$ ethanol at day 0 . Therefore, it is possible that ethanol or TPM concentrations fell over time due to metabolism by the cells. To determine if the cultured cells are metabolizing the active compounds within the TPM samples would be problematic, as the active MAO inhibitors need first to be identified. However, to determine if the general TPM sample is being metabolized by the cells, small aliquots of the culture media could be sampled at regular time intervals, and assayed by GC-MS for nicotine, cotinine, or tobacco-specific nitrosamine concentrations. 
It is also possible that healthy cells exposed to TPM for three days up-regulated MAO protein levels in response to inhibition by the TPM, but cells exposed for five days began to suffer from nutrient depletion and accumulation of metabolites. These factors may have interfered with the cells' ability to respond to TPM in a similar manner to healthier cells. In fact, the present study did find some evidence that TPM and QTPM samples induced an up-regulation of MAO gene expression, which will be discussed in Chapter Six of this thesis.

In an effort to ensure that the concentration of TPM compounds remained stable over the treatment period, SH-SY5Y cells were also exposed to TPM by a refreshed 5-day treatment regimen in which the culture medium was replaced with fresh medium after 3 days incubation, and a fresh aliquot of TPM was added. The cells were then incubated for a further two days before harvesting. Unexpectedly, total MAO activity increased in cells treated with this regimen. This apparent stimulation of MAO activity is difficult to explain, but it is evident that the cell culture conditions have a crucial effect on MAO enzyme activity. Cells treated with tobacco compounds for five days continuously without a medium change will have been impacted by reduced nutrients and the accumulation of metabolites in the culture media, and are likely to be less healthy than cells treated with the refreshed medium regimen. The data gathered in the present study suggests that healthier, more nourished SH-SY5Y cells may respond to tobacco extract exposure by increasing MAO enzyme activity.

To provide a clearer picture of the response of cultured SH-SY5Y cells to tobacco compounds, it would be useful to eliminate the confounding effects of the cell culture conditions. This could be achieved by employing a continuous perfusion culture system, as described by Constantinescu et al. (2007). Constantinescu et al. maintained small cultures of differentiated SH-SY5Y cells for up to two months by using a peristaltic pump to constantly refresh the cell medium. However, these methods would be prohibitively expensive for a study like the present one, due to the large numbers of cells required for experiments. It would also be interesting to repeat the experiments in this chapter with fully differentiated SH-SY5Y cells, as these would be expected to have a more neuronal phenotype (Constantinescu et al., 2007; Pahlman et al., 1990), and may more accurately represent a model of human neurons.

Another possible explanation for the somewhat inconsistent MAO inhibition observed in SH-SY5Y cells could be contamination with other bio-active compounds from the 
disposable laboratory plasticware used in this study. McDonald et al. (2008) reported that plastic processing additives used in the manufacture of disposable plastic laboratory tubes can leach into biological media and solvents, and leachates from several brands of plastic tubes were found to significantly inhibit or significantly increase MAO-B enzyme activity. The present study ensured all plasticware used for experiments was clean and sterile, but made no effort to control the brand of plasticware used. It is unknown what effect, if any, the use of disposable plasticware may have had on the results of this study.

\subsection{4 - Effects of Tobacco Extract Exposure on Total MAO Activity in U-118 MG Cells}

Previous studies have reported that human glia and astrocytes exhibit high levels of MAO activity (Mazzio et al., 2005; Westlund et al., 1988). This study confirmed that MAO activity is present in cultured human U-118 MG glioma cells, although the activity was only $6 \%$ of the activity measured in undifferentiated SH-SY5Y neuroblastoma cells. The reactions with U-118 MG cell lysates gave very low fluorescence, near the limits of detection for this assay. Since previously reported evidence shows human glial cells to have a high level of MAO activity, this suggests that the U-118 MG cell line may not be a suitable model to investigate the behavior of MAO in glial cells. This is in agreement with Ekblom et al. (1993), who reported high levels of MAO-B activity in highly differentiated astrocytes in primary culture, but glioblasts showed very low MAO-B activity. In another study, Ekblom et al. (1996) measured MAO enzyme activity in human 1242 MG glioma cells and reported these cells had MAO-A and MAO-B activity of approximately 6.8 $\mathrm{nmol} / \mathrm{hr} / \mathrm{mg}$ protein and $3.6 \mathrm{nmol} / \mathrm{hr} / \mathrm{mg}$ protein, respectively. Mazzio et al. (2005) reported rat $\mathrm{C} 6$ glioma cells had a total MAO activity of approximately $30 \mathrm{nmol} / \mathrm{hr} / \mathrm{mg}$ protein. It would be useful to compare the total MAO activity in cultured U-118 MG cells to MAO activity measured in another immortalized human glioma cell line, although there are few reported studies examining MAO activity in cultured human glioma.

As described previously (section 5.1.1) Ekblom et al. (1996) used the $1242 \mathrm{MG}$ glioma cell line to investigate the effects of ethanol and other psychoactive drugs on MAO enzymes in culture, and reported a significant increase in MAO-B activity. However, this cell line was not available to the experimenter, and no supplier could be found. The C6 rat glioma cell line is a more commonly used model of astrocyte and glial function, and 
Mazzio et al. (2005) demonstrated that cigarette smoke samples inhibited MAO in this cell line. However, C6 rat glioma cells were not used in the present study because it is not a human cell line. Studies have shown that the biochemistry of animal-derived cell lines differ considerably from human cell lines (Constantinescu et al., 2007), and C6 cells have been shown to exhibit marked differences from human glia (Olson et al., 1992). The present study aimed to use the cell models most relevant to human smoking.

Treatment of U-118 MG cells with nicotine, HTPM, and QTPM for 5 days had no significant effect on MAO activity in these cells. However, because MAO activity in this cell line was near the limits of detection of the kynuramine assay, it is possible that some inhibition of MAO may have occurred but was not detected by this assay.

\section{$\underline{5.4 .5-\text { Summary }}$}

Consistent with the results reported in Chapter Four of this thesis, both HTPM and denicotinized QTPM significantly inhibited total MAO activity in SH-SH5Y cells. However, inhibition of MAO seemed to be highly dependent on the cell culture conditions, and the results suggest that SH-SY5Y cells may up-regulate MAO activity or expression in response to MAO inhibition. While MAO activity was detected in the $\mathrm{U}$ 118 MG cell line, activity was very low and no inhibition was observed. This suggests that this cell line may not be a suitable model for glial functioning, as MAO activity has previously been reported in human glia and astrocytes. 


\section{Chapter Six: \\ Tobacco extract exposure alters MAO gene expression}

A number of studies have investigated the effects of nicotine and tobacco smoke on the activity of MAO enzyme activity in humans and animal models of addiction; however, there has not yet been any significant investigation into the effect of nicotine or tobacco on the gene expression of MAO. As discussed in Chapter One, tobacco exposure causes inhibition of MAO-A and MAO-B enzyme activity, and it is also possible that changes in MAO gene expression may occur following exposure to tobacco smoke. The present study and several others have found that inhibition of MAO enzyme activity appears to be reversible; therefore, changes in MAO gene expression may account for the longer term reductions in MAO activity observed in smokers.

Several microarray studies have investigated general changes in gene expression following treatment with nicotine, but none of these have examined MAO-A or MAO-B specifically. The present study aimed to characterize the gene expression of MAO-A and MAO-B in cultured SH-SY5Y cells in response to nicotine and tobacco smoke exposure, utilizing quantitative realtime RT-PCR.

\subsection{1 - MAO Gene Studies and Smoking}

Investigation of human MAO genes with respect to smoking has largely focused on identifying genetic markers of addiction. It has been proposed that genetic factors account for up to $70 \%$ of the likelihood of becoming a smoker (Heath et al., 1999), and this genetic predisposition is most likely determined by multiple genes. It has been theorized that individual differences in MAO enzyme expression and activity may contribute to genetic susceptibility to becoming a smoker and could influence the ability of a smoker to quit. These differences may be partly determined by genetic polymorphisms in the MAO genes. Several polymorphisms of the MAO-A and MAO-B genes have been identified in humans, and these polymorphisms appear 
to have some functional significance. It has been suggested that genetic polymorphisms may be relevant to the etiology of smoking behaviour, causing differences in individual MAO protein levels and activity. Some evidence has been reported that both high and low levels of MAO may influence smoking and quitting behaviors (J. Harro et al., 2004).

A variable number of tandem repeat (VNTR) polymorphism has been identified in the human MAO-A promoter region that affects transcription of the gene (Ito et al., 2003). The polymorphism is located $1.2 \mathrm{~kb}$ upstream of the MAO-A protein coding region, and alleles have been found with $1,2,3,3.5,4$ or 5 copies of a 30 bp repeated sequence. These alleles display differing frequencies between different ethnic groups. Alleles with 3.5 or 4 repeats are transcribed with greater efficiency than other alleles, suggesting there is an 'optimum length' of the MAO-A promoter. This polymorphism may play a role in the susceptibility of an individual to cigarette addiction, since a reduced risk of being a smoker was reported for females carrying the 4 repeat allele; whereas, males with the 4 repeat allele showed increased risk of being a smoker. An explanation of these apparently contradictory findings is not yet available.

Another polymorphism at position 644 in intron 13 of the human MAO-B gene has been associated with significantly reduced enzyme activity in the human brain, but increased activity in blood platelets. This polymorphism involves the substitution of an adenine base at position 644 for a guanine base (Checkoway et al., 1998). The $G$ allele also displays differences in allele frequency between ethnic groups, with $20 \%$ of Asian populations and $40 \%$ of Caucasian populations displaying this allele (Tan et al., 2003). The $G$ allele has greater transcriptional activity than the A allele (Costa-Mallen et al., 2005) and has also been associated with a slightly increased risk of Parkinson's disease in Caucasian populations (Tan et al., 2003). A recent study investigating this polymorphism in conjunction with the TaqlB polymorphism in the dopamine D2 receptor gene (DRD2) found a strong association between the DRD2/644A haplotype and smoking behavior in men (Costa-Mallen et al., 2005). It was reported that $82 \%$ of men with this haplotype were current or ex-smokers, compared to $46 \%$ of men with the same DRD2 allele and the $644 \mathrm{G}$ allele. A convincing case was made for an interaction between these two genes in predisposing to smoking behaviour in men. Interpretation of the results of these studies is complex, since the polymorphism occurs in the non-coding region of the gene and does not affect the amino acid sequence of the protein (Checkoway et al., 1998). An explanation for how this polymorphism may alter MAO-B gene expression has not been proposed. 
Additionally, the VNTR and 644G polymorphisms may act together to influence smoking behaviour. The association between MAO-A/B haplotype and smoking behavior has been examined, and some evidence exists to suggest that both polymorphisms may predispose to the smoking habit. Ito et al (2003) investigated this link in a Japanese population and found that males with a 3-repeat/644G haplotype began smoking at a later age than males with other haplotypes, and men with the 4-repeat allele were found to have a significantly increased risk of nicotine dependence. Further investigation is required to confirm the association between these alleles and an increased risk of smoking behavior, and to identify the mechanisms by which these polymorphisms influence transcription of MAO-A and $B$ genes.

While a significant amount of research has been conducted into the genetic differences between smokers, the effects of nicotine or tobacco smoke exposure on the expression of MAO-A or MAO-B genes has yet to be examined. Tobacco smoke is a mixture of over 4000 different compounds, and it is likely that some of these compounds can alter the expression of the MAO-A or MAO-B genes. Considering the likely involvement of MAO in the establishment and maintenance of tobacco dependence, it is possible that any alteration in MAO gene regulation or expression induced by tobacco smoke constituents will influence the role that MAO plays in the cell's response to tobacco. As such, this area presents an important gap in the current understanding of tobacco and MAO interaction.

\section{$\underline{6.1 .2-N i c o t i n e ~ a n d ~ G e n e ~ E x p r e s s i o n ~}$}

Nicotine is known to modulate the expression of many genes involved in diverse cellular functions (Dunckley \& Lukas, 2003, 2006). Some of these include tyrosine hydroxylase, which is involved in catecholamine synthesis, and the immediate early genes c-fos, c-jun, and junB (Pelto-Huikko et al., 1995). These immediate early genes act as transcription factors and may lead to nicotine-induced regulation of many genes (Dunckley \& Lukas, 2003).

A study into the effects of nicotine on SH-SY5Y cells used microarray technology to identify novel nicotine-regulated genes in SH-SY5Y cells (Dunckley \& Lukas, 2003). A gene library of approximately 5000 fluorescently labeled cDNA transcripts was assembled from untreated SHSY5Y cells, and from cells treated with $1 \mathrm{mM}$ nicotine for 1 hour. Nicotine consistently altered the expression of 17 different genes. It was noted that the gene expression changes identified 
were of generally low magnitude, showing up-regulation of only $26-38 \%$, and down-regulation of $21-55 \%$. RT-PCR was used to confirm the results of the microarray analysis, and to determine whether nAChR activation was required to regulate these genes. SH-SY5Y cells were then treated with nicotine in the presence of d-tubocurarine (d-TC), a non-specific nAChR antagonist, and assayed by RT-PCR. Treatment with d-TC blocked or reversed the nicotine-induced changes in mRNA expression for all of the genes tested. The authors concluded that nAChR activation was required for the nicotine-dependent modulation of gene expression, and that these changes in gene expression were a result of nicotine binding to the nAChRs.

In a later experiment by the same group, microarray analysis was used to identify changes in gene expression arising from longer-term exposure to nicotine in SH-SY5Y cells (Dunckley \& Lukas, 2006). Cells were treated with $1 \mathrm{mM}$ nicotine for 24 hours, and microarray analysis used to identify changes in expression of at least 160 different genes in this cell line. These effects were observed in genes involved in many diverse cellular functions, including binding, metabolism, catalytic activity, and physiological processes. Some of the genes affected included eukaryotic translation initiation factor $4 \mathrm{~A}$ isoform 2 (elF4A2), contactin 1, and son of sevenless homolog 1 (SOS1), which were up-regulated, and ubiquitin-conjugating enzymes, glyceraldehyde-3-phosphate dehydrogenase (GAPDH), stress-induced-phosphoprotein 1 (STIP1) and Parkin which were down-regulated. Once again, real-time PCR was used to determine if the observed changes in gene expression were dependent on the activation of nAChR-mediated signaling pathways, this time using the nAChR antagonists $\alpha$-cobratoxin and mecamylamine, in addition to d-TC. It was confirmed that in most cases nAChR antagonists were able to reverse the alteration in gene expression induced by nicotine alone, suggesting that observed changes in gene expression were a result of nAChR-mediated signaling pathways, and dependent on nicotine binding.

The results reported in these experiments indicate that nicotine exposure can modulate the expression of a diverse range of genes with varied functional significance. However to date, no studies have specifically investigated the effects of nicotine or tobacco on MAO-A or MAOB transcription. 


\subsection{3 - Quantitative Real-Time PCR}

Reverse transcription real-time PCR has proven to be one of the most sensitive, accurate, and rapid methods for the quantitative analysis of gene expression. It offers several advantages over other gene expression methodologies like Northern blot analysis, reporter gene transfection, or end-point PCR, as it shows greater sensitivity, better reproducibility and is less labour intensive. The high sensitivity of real-time RT-PCR allows quantification of lowabundance transcripts and accurate detection of subtle changes in gene expression.

The protocol for a quantitative RT-PCR reaction involves isolating mRNA from biological samples and first performing a reverse transcription reaction to transcribe the mRNA to CDNA. The cDNA is then used as a template and amplified by PCR. Real-time QPCR instruments monitor the accumulation of amplicon in the PCR reaction after each successive cycle with the use of fluorescent probes and dyes such as the intercalating dye SYBR Green I. SYBR Green I binds specifically to the minor groove in the double stranded DNA helix, and fluoresces intensely when bound. As the PCR reaction progresses the concentration of double-stranded DNA increases causing a corresponding increase in the intensity of SYBR Green I fluorescence.

A PCR is characterized by three phases of amplicon accumulation. The exponential phase occurs at the start of the reaction when reagents are not limiting and the concentration of PCR product increases exponentially. This is followed by the linear phase, when product formation slows to a linear increase as PCR reagents become limited. As the reaction progresses some reagents will become depleted or the polymerase enzyme may become inactivated. At this point no more PCR product will be formed, and the PCR will reach the plateau phase. During the exponential phase fluorescence intensity increases proportionally as the amount of amplicon doubles with each successive cycle of the reaction. It is in this phase of the reaction that real-time qPCR instruments measure the accumulation of fluorescence. The first cycle at which the fluorescence produced by target amplification can be distinguished from baseline fluorescence is termed the threshold cycle or $\mathrm{Ct}$, and is the main metric unit used in real-time PCR analysis. The Ct value can be directly correlated to the initial target concentration in the reaction - the greater the amount of initial target CDNA, the earlier the Ct value for that sample (Fig. 38a). The qPCR fluorescence threshold is usually set by the proprietary software used to operate each real-time PCR instrument and is based on the level of baseline fluorescence. The fluorescent threshold is set at a point where all samples show an exponential increase in fluorescence that can be distinguished from background fluorescence (Yuan et al., 2006). 
This methodology has been adapted to provide two different methods of quantification: absolute quantification and relative quantification. Absolute quantification is used when a measure of the exact copy number of template is required (for example, measuring viral load); whereas, relative quantification is used for most studies of gene expression. Relative quantification measures the relative concentration of a gene of interest (GOI) compared to the concentration of that gene in a calibrator or control sample (e.g. an untreated sample). By comparing gene expression in samples to a control sample, the fold-change in gene expression can be ascertained. To control for variation in RNA isolation and efficiency of the reverse transcription reaction, data for samples and the calibrator are normalized to the expression of a house-keeper or reference gene. An appropriate reference gene is a gene that shows constant, stable expression under the experimental conditions of the assay. Commonly used references include GAPDH, $\beta$-actin, and 18s rRNA; however, these have been shown to vary considerably under some experimental conditions, and so it is necessary to validate the stability of proposed reference genes. After stability of the reference gene has been confirmed, any variances in the $\mathrm{Ct}$ of the reference gene between samples can be attributed to differences in the RT reaction, or the quality of the mRNA added. These sources of variation will affect the $\mathrm{Ct}$ of the $\mathrm{GO}$ and the reference gene equally, so differences in the $\mathrm{Ct}$ of the reference gene between samples can be used to normalize the data - a step that is vital to the accuracy of gene expression measurement.

The design of an accurate and sensitive assay for the measurement of mRNA expression levels requires a number of important considerations and optimizations to eliminate variation and produce meaningful data. The first of these is the extraction and transcription of mRNA. Several different methods of priming the reverse transcription reaction have been described: random hexamer primers, oligo-dTs, and gene-specific primers. Random hexamers anneal at many locations along each RNA template, and will ensure transcription of a wide range of cDNAs. However, random hexamers will produce more than one cDNA sequence for each RNA target and will also synthesize cDNA from ribosomal RNA. If the mRNA target of interest is expressed at low levels, the cDNA may not be expressed proportionately (Bustin et al., 2005). Oligo-dTs offer more specific priming than random hexamers, as they anneal to the poly-A tails on mRNAs. Priming with oligo-dTs ensures that an accurate cDNA copy of the mRNA pool is generated. It is also useful when amplifying several mRNA targets from a single sample when the sample is limited. However, oligo-dT priming may generate a 3' bias in the cDNA, since very long mRNAs may not be transcribed completely. This can be problematic if PCR primer binding sites are located at the $5^{\prime}$ end of the cDNA. Accurate generation of CDNA 
using oligo-dTs also requires full-length RNA of good quality, so it is good practice to test the integrity of extracted RNA when using oligo-dTs. Gene specific primers offer the most specific option for cDNA priming, but because an RT reaction needs to be performed with different specific primers for each gene of interest, this method can be quite wasteful when working with limited samples or multiple GOls.

Another vital consideration in the optimization of an accurate qPCR assay is the efficiency with which the customized primers are priming the PCR reaction. The basic principle used to analyze real-time PCR data assumes that the number of cycles required to reach threshold fluorescence correlates with initial target concentration, and this can only be accurately measured if the PCR reaction is operating at $100 \%$ efficiency - that is, when each PCR cycle results in a doubling of the target amplicon.

(a)

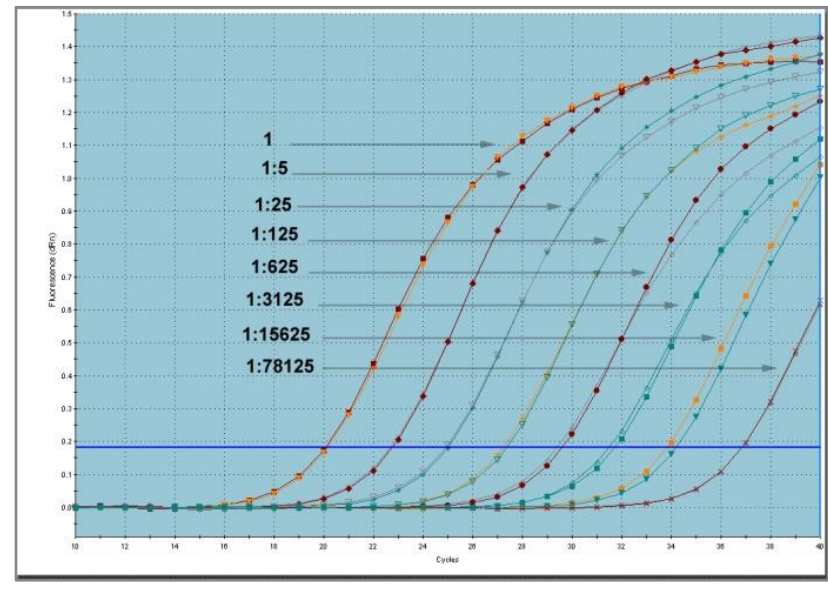

(b)

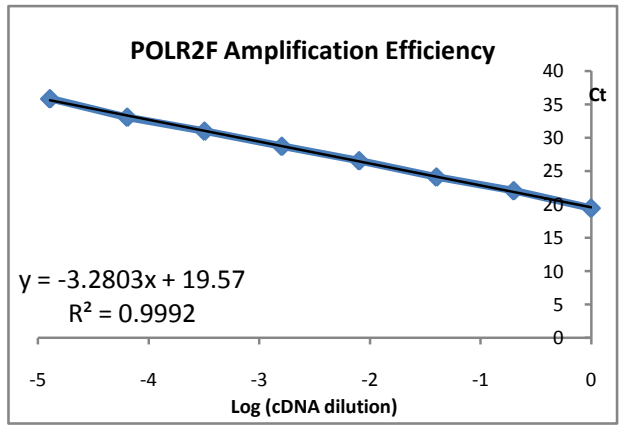

Figure 38: (a) SYBR Green I fluorescence versus Cycle number for a serially diluted sample of SH-SY5Y CDNA amplified using the POLR2F specific primers. Each dilution was assayed in duplicate. Note the more dilute samples reach threshold fluorescent (blue horizintal line) after a greater number of amplification cycles. These data can be used to derive an amplification efficiency plot (b), the slope of which is used to determine the percentage efficiency of the amplification reaction. In this case, the reaction primed with the POLR2F primers procedes with an efficiency of $101.8 \%$.

The efficiency of a qPCR reaction can be determined by constructing a standard curve using a positive control template (Fig. 38b). Generally, a sample positive for the template of interest is serially diluted over several orders of magnitude encompassing the expected range of the experimental samples and then amplified using the experimental PCR protocol and customized primers. The $\mathrm{Ct}$ value of each dilution is plotted against the logarithmtransformed template dilution, and should yield a linear fit. When a base 10 logarithm is used, the slope should be between -3.1 and -3.6 , indicating $90-110 \%$ reaction efficiency. A 
low reaction efficiency may be corrected by optimizing the primer or magnesium concentration; whereas, efficiency values above $100 \%$ may indicate the formation of primer dimers, or non-specific amplification.

The presence of non-specific PCR products or primer dimers can be checked using a PCR product melt curve analysis after every PCR run. Analysis of the melt curve can determine if anything other than the gene of interest is amplified. A melt curve is generated by the qPCR instrument which anneals all PCR products at $55^{\circ} \mathrm{C}$ and incrementally increases the temperature while recording the changes in fluorescence intensity. Fluorescence intensity of the SYBR Green I dye will fall rapidly at the temperature at which the double-stranded PCR product dissociates. These data can be used to plot raw fluorescence against temperature, or more commonly the negative first derivative of raw fluorescence versus temperature. This second plot will show the melting temperatures of different PCR products as individual peaks located at distinct temperatures. A specific and well-optimized GPCR assay will show the presence of a single, narrow, homogeneous peak. Primer dimers and non-specific amplification products can be identified by the presence of additional peaks on the melt curve located at different temperatures. Because SYBR Green I binds to all double-stranded DNA the presence of non-specific products in a qPCR reaction render the assay inaccurate, and the Ct data cannot be trusted for quantification purposes.

An ideal qPCR assay requires careful optimization and validation, but will amplify a single specific PCR product reliably and reproducibly. Once these results are achieved, qPCR can be used to sensitively and quantitatively detect changes in gene expression.

\subsubsection{1 - Data Manipulations and Calculations}

Determining fold-changes in mRNA expression using quantitative RT-PCR requires some calculation and data manipulation of the $\mathrm{Ct}$ metric recorded. A commonly used transformation of the data is the $2^{-\Delta \Delta C t}$ method (Livak \& Schmittgen, 2001). This method is used to calculate relative changes in gene expression by comparing the $\mathrm{Ct}$ values of the $\mathrm{GOI}$ in the treated sample with the $\mathrm{GOI}$ in the calibrator sample (untreated control) and uses the $\mathrm{Ct}$ values of the reference gene in each to normalize the data. 
The first calculation made is $\Delta \mathrm{Ct}$, which is defined as the threshold cycle of the gene of interest, minus the threshold cycle of the reference gene, or

$$
\Delta \mathrm{CT}=\mathrm{CT}_{\mathrm{GOI}}-\mathrm{CT}_{\mathrm{REF}}
$$

From here, the difference in $\Delta \mathrm{Ct}$ between the unknown sample and the calibrator (or untreated) sample are compared as

$$
\Delta \Delta \mathrm{CT}=\Delta \mathrm{CT}_{\mathrm{UNK}}-\Delta \mathrm{CT}_{\mathrm{CAL}}
$$

The negative reciprocal of the $\Delta \Delta \mathrm{Ct}$ figure is taken and raised as an exponent of 2 to give the quantitation of the target, normalized to an endogenous reference gene relative to a calibrator (Livak \& Schmittgen, 2001). This figure is known as the Relative Quantitation or RQ, and expresses fold change in gene expression relative to the calibrator. For example, a sample with an RQ of 1.0 shows the same level of gene expression as the calibrator. An RQ of 0.5 indicates a sample with $50 \%$ less expression than the calibrator; while a sample with an $\mathrm{RQ}$ of 1.75 has $75 \%$ more expression than the calibrator.

However, for the $2^{-\Delta \Delta \mathrm{Ct}}$ calculation to be valid, the amplification efficiencies of the $\mathrm{GOI}$ and reference gene primers are assumed to be equal, and near $100 \%$. When amplification efficiencies are unequal or significantly below $100 \%$ the calculated RQ could be very different from actual gene expression. As the PCR efficiencies of the primers used in this study were not equal, a modified analysis of the $2^{-\Delta \Delta C t}$ method was used to correct for this (Yuan et al., 2006; Yuan et al., 2008). The efficiency-adjusted $\Delta \Delta C \mathrm{C}$ method uses many of the same calculations as the original $2^{-\Delta \Delta C t}$ approach but allows correction for differences in the PCR efficiency of the reactions. Thus, this method uses the same calculation for $\Delta \Delta \mathrm{Ct}$ described above, but first corrects each $\mathrm{Ct}$ value with the Percentage Amplification Efficiency (PAE). Therefore, the equation for the efficiency adjusted $\Delta \Delta \mathrm{Ct}$ method is:

$$
\Delta \Delta \mathrm{CT}_{\text {ADJUST }}=\left(\left(\mathrm{CT}_{\text {GOI_UNK }} \times P A E_{G O I}\right)-\left(\mathrm{CT}_{\mathrm{REF}_{-} \mathrm{UNK}} \times P A E_{\text {REF }}\right)\right)-\left(\left(\mathrm{CT}_{\mathrm{GOI}_{C} \mathrm{CAL}} \times P A E_{G O I}\right)-\left(\mathrm{CT}_{\text {REF_CAL }} \times P A E_{\text {REF }}\right)\right)
$$

PAE is estimated by linear regression of an efficiency standard curve, described above. $\mathrm{Ct}$ is plotted against the logarithm-base-2 transformation of the concentration, and the slope of a line fit to this plot is the PAE.

The efficiency-adjusted $\Delta \Delta \mathrm{Ct}$ value can then be transformed into the Relative Quantitation (RQ) figure as described above. 


\section{$\underline{6.1 .4-O b j e c t i v e s}$}

The aim of the present study was to use quantitative real-time PCR to investigate the effects of nicotine and tobacco extracts on the expression of MAO-A and MAO-B genes in cultured SH-SY5Y human neuroblastoma cells.

Cultured SH-SY5Y cells were exposed to nicotine, HTPM, and QTPM over a number of different treatment regimens before harvesting the total RNA from the cells. The mRNA was transcribed to cDNA and assayed by real-time PCR. It was proposed that non-nicotine compounds within TPM may down-regulate the expression of MAO-A or MAO-B, contributing to the inhibition of these enzymes observed in chronic human smokers. If this hypothesis is correct, it was expected that exposure to denicotinized TPM (QTPM) would induce downregulation of the MAO-A or $-B$ genes, similar to that seen after exposure to standard HTPM. Based on the literature, nicotine alone was not expected to change MAO-A or MAO-B gene expression.

The approach used by Dunckley \& Lukas (2006) was adapted to determine if changes in MAOA gene expression following HTPM exposure were independent of any action of nicotine on the cells. Cultures of SH-SY5Y were also exposed to HTPM and denicotinized QTPM in the presence of mecamylamine, a non-specific nAChR antagonist. As discussed in section 6.1.2, Dunckley \& Lukas used mecamylamine to determine the nAChR-dependent basis of changes in expression of a number of genes. The present study aimed to use mecamylamine inhibition to investigate the nature of the changes in MAO-A and MAO-B gene expression specifically. It was expected that changes in MAO gene expression in SH-SY5Y cells would be independent of the actions of the nAChRs, since no direct interaction between nicotine and MAO-A or MAO-B has previously been reported. Therefore, mecamylamine inhibition of nAChRs should have no effect on the changes in gene expression observed in response to HTPM or denicotinized QTPM.

Thus the overall aims of this study were twofold: to investigate whether nicotine or tobacco extracts altered the expression of MAO-A or MAO-B, and to determine if any changes observed were dependent on $\mathrm{nAChR}$ activation. 


\section{$\underline{6.2 \text { - MATERIALS AND METHODS }}$}

\subsection{1 - Tobacco Particulate Matter Exposures}

The expression of MAO-A and MAO-B mRNA was investigated in SH-SY5Y and U-118 MG cells following exposure to tobacco compounds. Cells were grown and maintained according to the protocol described in section 2.1. Several different treatment regimens were used to study the cellular responses to these exposures, as detailed below.

\subsubsection{1 - MAO Gene Expression in SH-SY5Y Cells}

Batches of five $600 \mathrm{~mL}$ culture flasks of SH-SY5Y cells were sub-cultured at low density and grown to near confluence in the presence of ethanol, purified nicotine, TPM from standard cigarettes (HTPM), and TPM from denicotinized tobacco (QTPM). The cells received nicotine or HTPM to a total final concentration of $0.2 \mu \mathrm{M}$ nicotine, and equivalent volumes of ethanol or QTPM were used (9.8 $\mu \mathrm{L}$ per $30 \mathrm{~mL}$ of media; $0.03 \%$ ethanol). These extracts were added directly to the cell culture medium, and the cells were maintained in this exposure medium without disruption for 1,3 , or 5 days. After harvesting the cells, total RNA was extracted according to the methods described in section 2.6.1, and cell samples were assayed for changes in gene expression.

For this experiment, 4 flasks each of a) ethanol-exposed, b) nicotine-exposed, c) HTPMexposed, and d) QTPM-exposed cells were treated for 1 day before harvesting and RNA extraction. Seven flasks of each treatment group were exposed for 3 days, and 16 flasks of each treatment group were prepared for the 5-day time point. The 5-day exposures were expected to show the maximal response, and therefore more replicates of this treatment were prepared.

The mRNA extracted from these treated cells was transcribed into cDNA according to the protocol described in section 2.6.2, and assayed by real-time PCR using the methods described in 2.6.3. Each sample was assayed in duplicate at least twice, and the mean Ct value of these replicates was used for the analysis. 


\subsubsection{2 - MAO Gene Expression in U-118 MG Cells}

Changes in MAO-A and MAO-B gene expression in U-118 MG cells were also investigated. Cells were cultured according to the methods described in section 2.1. U-118 MG gene expression was examined at the continuous 5-day treatment regimen as it was hoped it would show the maximal response to treatment.

For this experiment, 8 flasks of each treatment condition were exposed to ethanol, nicotine, HTPM, and QTPM. Nicotine and HTPM were added to a final nicotine concentration of 0.2 $\mu \mathrm{M}$, and ethanol and QTPM were added at equivalent volumes, as described above. The cells were exposed for 5 days continuously, without disruption and without any changes of the medium. This is the same regimen used to treat SH-SY5Y cells exposed for 5 days continuously.

At the completion of the treatment regimen the cells were harvested, and the total RNA was extracted and transcribed to cDNA according to the methods described in section 2.6.2. The resulting CDNA samples were then assayed by real-time PCR in duplicate, on two separate occasions.

\subsubsection{3 - Gene Expression in SH-SY5Y Cells Treated with Refreshed Media}

Analysis of the data obtained from the 1,3 , and 5 day exposure groups of SH-SY5Y cells showed that many treatments induced a change in gene expression after 3 days treatment, yet the expression fell back to near control levels after 5 days treatment. There was some concern that this might indicate that the ethanol, nicotine, HTPM, and QTPM compounds were being metabolized and degraded by the cells. Hence, a fourth treatment regimen was subsequently added to the experiment, in which cultured SH-SY5Y cells were treated with ethanol, nicotine, HTPM and QTPM as in the previous experiment, but the medium in the flasks was replaced at day 3 . On day 3 , the culture medium was removed by aspiration and replaced with fresh culture medium supplemented with ethanol, nicotine, HTPM, or QTPM. Cell cultures were then returned to the incubator for a further 2 days before harvesting for total RNA. This treatment regimen was used to try to maintain the exposure conditions at a constant concentration. Six flasks of each treatment group were prepared for this experiment, and each sample was assayed for MAO-A and MAO-B gene expression in duplicate. 


\subsubsection{4-Mecamylamine Exposures}

In addition to the nicotine and TPM exposure regimens described above, SH-SY5Y cells were also treated with these tobacco compounds in the presence of the nAChR antagonist mecamylamine. Mecamylamine inhibits the action of all nAChR subunits non-specifically. Untreated stocks of SH-SY5Y cells were grown to near-confluence in culture medium and mecamylamine (reconstituted in distilled $\mathrm{H}_{2} \mathrm{O}$ ) was added to give a final concentration of 10 $\mu M$. Relevant volumes of nicotine, HTPM, and QTPM were also added to the medium to give final nicotine concentrations of $0.2 \mu \mathrm{M}$ or equivalent volumes of treatment, as described above. Control flasks receiving treatment with mecamylamine only, or mecamylamine in the presence of ethanol, were incubated in parallel with the nicotine and TPM treated samples.

After the addition of mecamylamine and tobacco compounds, the treated cells were incubated for 3 days undisturbed. At the completion of the exposures, the flasks of cells were prepared for total RNA extraction as described above.

\subsection{2 - Quantitative Real-Time PCR}

\subsubsection{1 - Reference Genes}

DNA-directed RNA polymerase II subunit F (POLR2F) was selected as a reference or housekeeping gene for all qRT-PCR experiments based on the findings of Hoerndli et al (2004), who presented a list of suitable reference genes for use in SH-SY5Y cells. The stable expression of this gene was verified against two other house-keeping genes: GAPDH and M-RIP. All three genes were amplified in SH-SY5Y cells exposed to all treatments over all treatment regimens according to the qRT-PCR protocol described in section 2.6 of this thesis. Gene expression was compared between genes and analyzed using geNorm v3.5 software.

\subsubsection{2 - Primer Efficiency}

To test the PCR efficiency of the selected primer pairs a standard curve was prepared for each pair using cDNA transcribed from $2 \mu \mathrm{g} / \mu \mathrm{L}$ total RNA from SH-SY5Y cells. SH-SY5Y cDNA was serially diluted with $\mathrm{dH}_{2} \mathrm{O}$ over the range $1-256$ fold. Dilutions of cDNA were amplified in duplicate using the relevant primer concentrations according to the protocol described in section 2.6 of this thesis. 
Upon completion of the assay, Microsoft Excel 2007 was used to plot the mean Ct values for each dilution against the logarithm-base-10 transformed dilution factor. Linear regression analysis was used to fit a straight line to the data. The PCR efficiency was calculated from the slope of this line using the equation:

$$
\text { Efficiency }=10^{(-1 / \text { slope })}-1
$$

where slope refers to the slope of the plot of the logarithm-base-10 transformed template dilution versus $\mathrm{Ct}$ value (Peters et al., 2004) described in section 6.1.3.

\subsubsection{3 - Data Manipulation}

The adjusted $\Delta \Delta \mathrm{Ct}$ method described in section 6.1.3.2 was used to calculate $R Q$ for all the quantitative RT-PCR experiments in this thesis. This method was chosen to correct for differences in amplification efficiency between primers to achieve a more accurate picture of the changes in gene expression observed. The reference gene used for all experiments was POLR2F. For analysis of the effects of ethanol, gene expression in ethanol-treated cells was compared to that in untreated cells which served as the calibrator. When gene expression in nicotine, HTPM, or QTPM treated cells was analyzed, ethanol treated cells were used as the calibrator. Because nicotine, HTPM and QTPM samples all contained ethanol, the ethanol treated cells were used as the calibrator to control for any effects of the ethanol vehicle.

\subsection{3 - Western Blotting}

The abundance of MAO-A and MAO-B following nicotine and TPM treatments were estimated from Western blots of the cell extracts. Western blotting was performed on cell protein lysates collected at the same time the cell cultures were harvested for total RNA. These protein lysates were probed for MAO-A and MAO-B-specific immuno-staining and analyzed by densitometry on a semi-quantitative basis. This was to determine if observed changes in MAO gene expression were correlated with corresponding changes in MAO protein abundance. Lysates prepared from cells receiving the 5-day refreshed treatment regimen were used for this experiment, as these cells showed the greatest change in MAO gene expression. 
Some flasks exposed using the 5 day refreshed regimen did not yield enough cells for both RNA extraction and preparation of a protein lysate, and so the sample group available for Western blotting was smaller than that used for quantitative RT-PCR. Four samples each of ethanol, nicotine, HTPM, and QTPM treated cells were assayed by Western blot. One of each treatment was assayed per blot, for a total of four independent experiments.

Western blots for MAO-A were immunostained using a rabbit polyclonal antibody (Santa Cruz Biotechnology Inc; sc-20156) raised against amino acids 458 - 527 of the human MAO-A protein. MAO-B protein was detected using a goat polyclonal antibody (Santa Cruz Biotechnology Inc; sc-18401) specific for a C-terminus peptide sequence of human MAO-B. A mouse polyclonal anti- $\beta$-tubulin antibody (BD Pharmingen; cat. 556321) was used on both MAO$A$ and MAO-B blots to serve as a protein loading control. The MAO-A antibody produces a specific band at $61 \mathrm{kDa}$, the MAO-B antibody yields a $60 \mathrm{kDa}$ band, and the $\beta$-tubulin antibody produces a band at $50 \mathrm{kDa}$. MAO-A and MAO-B were visualized with $\mathrm{Cy}^{\circledR}-5$ labeled anti-rabbit (Jackson ImmunoResearch Inc; 711-175-152) and anti-goat (Jackson ImmunoResearch Inc; 705-175-147) secondary antibodies respectively, and $\beta$-tubulin was visualized with a $\mathrm{Cy}^{\circledR}{ }^{-5}$ labeled anti-mouse antibody (Jackson ImmunoResearch Inc; 715-175-150). The fluorescence from these was measured using the Fujifilm FLA-5100 imaging system (Fuji Photo Film Co., Ltd) with a $635 \mathrm{~nm}$ laser and the DBR1/R665 emission filter. Densitometry was performed with ImageJ v3.5 software (Wayne Rasbond, National Institutes of Health, USA) using an integrated pixel density method. Specific Western blotting procedures are detailed in section 2.5 of this thesis.

\section{$\underline{6.3-\text { RESULTS }}$}

\subsection{1 - Quantitative Real-Time PCR Validation}

\subsubsection{1 - Primer Efficiency}

Efficiency of the PCR reaction with all primer pairs was good and did not vary significantly from $100 \%$ (Table 13). PCR primers which give a reaction efficiency over $90 \%$ are preferred for quantitative analysis; whereas, efficiencies above $100 \%$ indicate the formation of primer 
dimers. Variations in amplification efficiency between primer pairs were corrected for in the analysis of the real-time PCR data using the adjusted $\Delta \Delta C$ t method described in section 6.1.3.2.

\subsubsection{2 - Primer Specificity}

The dissociation curves for the PCR products produced from each primer pair all yielded a single, specific peak (Table 13), indicating the PCR reactions each amplified only a single specific product.

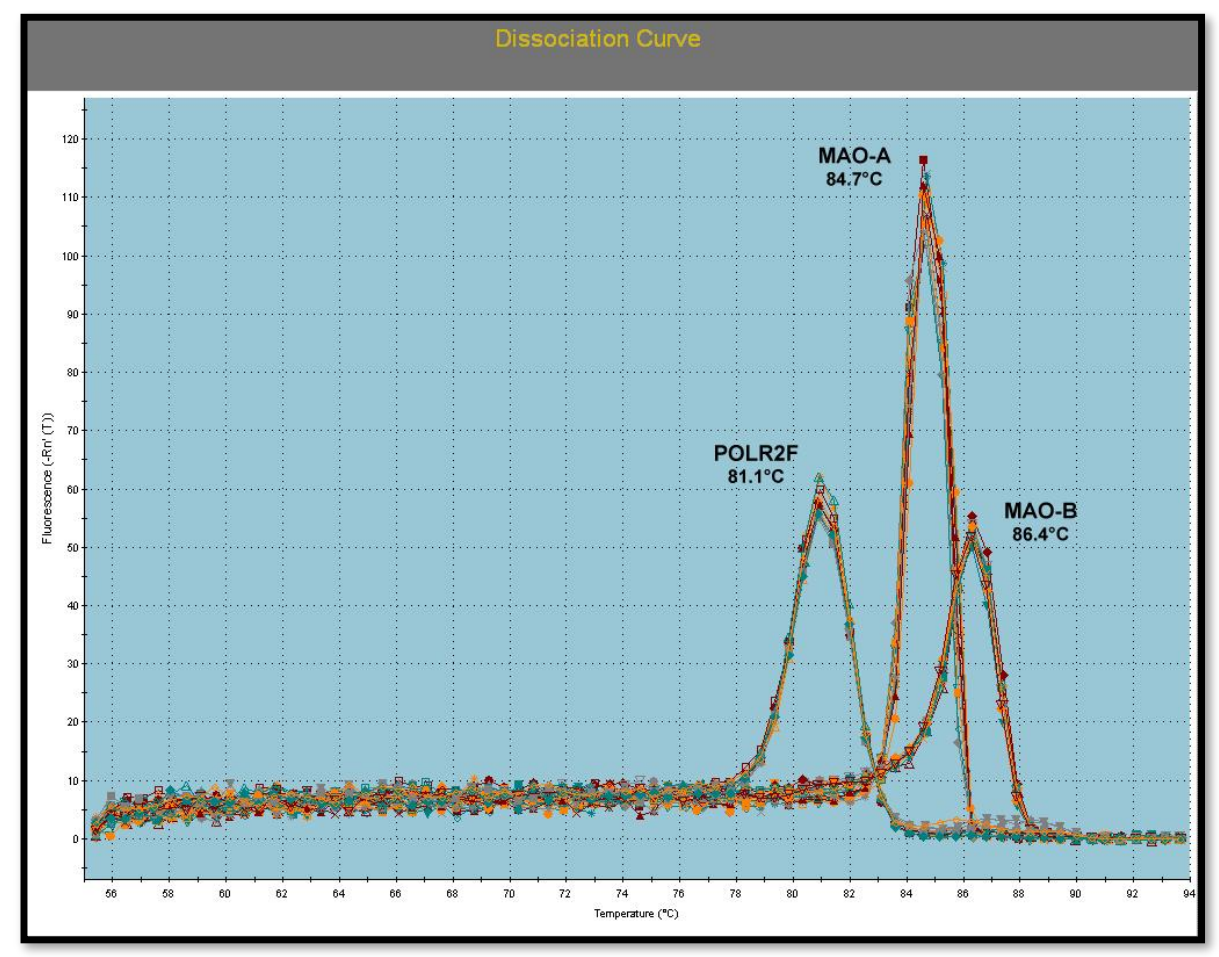

Figure 39: Representative dissociation curves demonstrating melt peaks for POLR2F, MAO-A, and MAO-B genes amplified in SH-SY5Y cells.

Melt curve analysis of negative-control PCR reactions was also performed to determine if contamination by PCR product or genomic DNA had occurred. Negative control reactions in which cDNA template was replaced with an equivalent amount of $\mathrm{ddH}_{2} \mathrm{O}$ were performed with every assay, and none displayed any PCR-product fluorescence. 
Table 13: Efficiency of primer pairs used for qRT-PCR

\begin{tabular}{lcc|lccc}
\hline & \multicolumn{4}{c}{ Target genes } & \multicolumn{4}{c}{ Reference genes } \\
Primer Pair & PCR Efficiency $(\%)$ & $\mathrm{Tm}\left({ }^{\circ} \mathrm{C}\right)$ & \multicolumn{1}{l}{ Primer Pair } & PCR Efficiency $(\%)$ & $\operatorname{Tm}\left({ }^{\circ} \mathrm{C}\right)$ \\
\hline MAO-A & 91.4 & $84.7^{\circ}$ & POLR2F & 101.8 & $81.1^{\circ}$ \\
MAO-B & 100.0 & $86.4^{\circ}$ & GAPDH & 96.6 & $81.3^{\circ}$ \\
elF4A2 & 99.5 & $82.6^{\circ}$ & M-RIP & 108.0 & $83.7^{\circ}$ \\
\hline
\end{tabular}

Amplification efficiencies and PCR product melt peaks are presented for all primer pairs used in this experiment.

PCR products from the real-time PCR reactions were also separated by electrophoresis on agarose gels to check primer specificity. In every case, each primer pair amplified a single PCR product, and these conformed to the sizes of specific amplicons predicted by BLAST genome analysis (section 2.6.4).

(a)

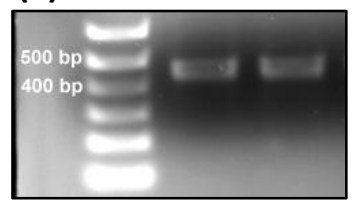

(b)

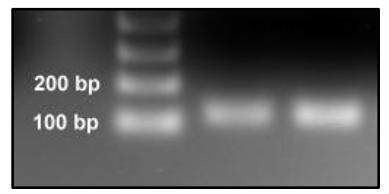

(c)

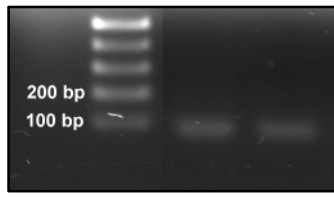

Figure 40: Electrophoretic bands of (a) MAO-A, (b) MAO-B, and (c) POLR2F PCR products following agarose gel electrophoresis and staining with ethidium bromide. The specific amplicon bands measure $457 \mathrm{bp}, 104 \mathrm{bp}$, and $65 \mathrm{bp}$, respectively. Each gel depicts one lane of $1 \mathrm{~Kb}+$ molecular weight ladder, and two lanes of PCR product. The PCR products were obtained from the GPCR reactions containing the intercalating dye SYBR Green I, and so ethidium bromide staining is relatively weak because the SYBR Green I dye competes with ethidium bromide.

The MAO-A PCR product showed a single electrophoretic band at approximately $450 \mathrm{bp}$ (Fig. 40a); the MAO-B product displayed a band of approximately 100 bp (Fig. 40b); and POLR2F primers yielded a single band at approximately $70 \mathrm{bp}$ (Fig. 40c). These results are in good agreement with the predicted amplicon (section 2.6.4). This further confirms that the designed PCR primers amplify a specific PCR product from the genes of interest.

\subsubsection{2 - Reference Genes}

SH-SY5Y and U-118 MG cells that had been exposed to various tobacco extracts were analyzed by quantitative real-time PCR. Gene expression of MAO-A, MAO-B, and elF4A2 was assayed in 
these cell lines relative to the expression of the reference gene POLR2F. The expression of POLR2F was compared to two other reference genes, GAPDH and M-RIP, to determine if POLR2F expression was stable following exposure to these tobacco extracts and to validate its use as a reference gene for this study.

(a)

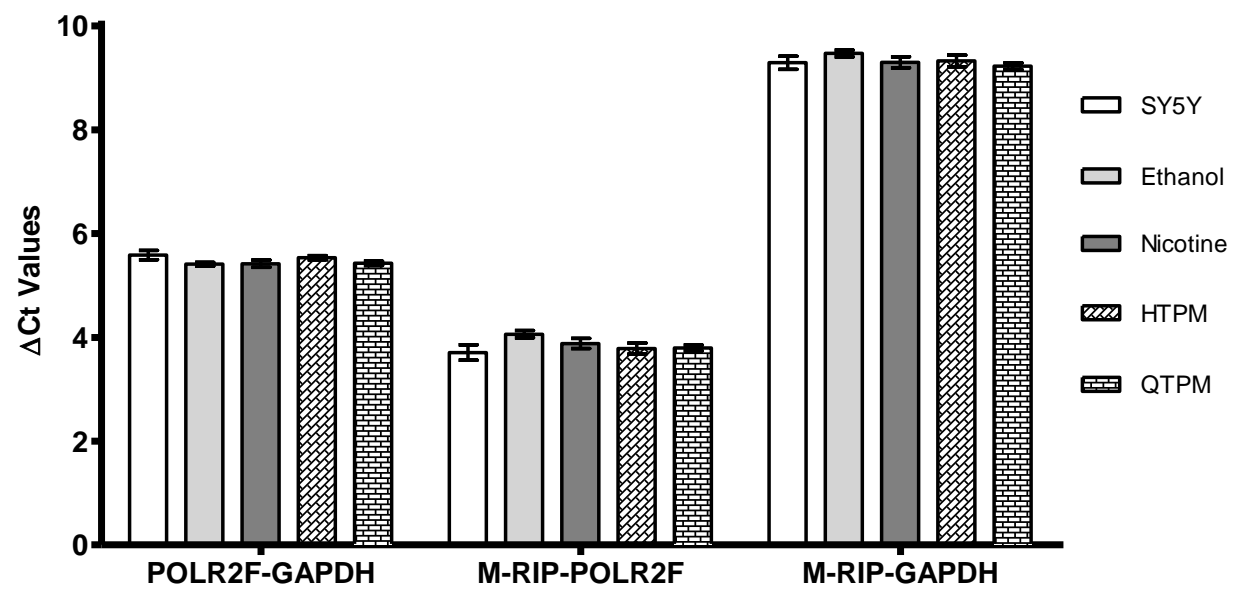

Figure 41a: Expression of the POLR2F reference gene was validated against the expression of two other reference genes - the commonly used house-keeper gene GAPDH, and M-RIP. Comparison of the expression of these three reference genes was made in all samples and treatments used in this study. No significant difference was found in the gene expression of any of these genes following exposure to any of the treatments. (a) presents the difference in $\mathrm{Ct}$ between pairs of primers for all treatments.

(b)

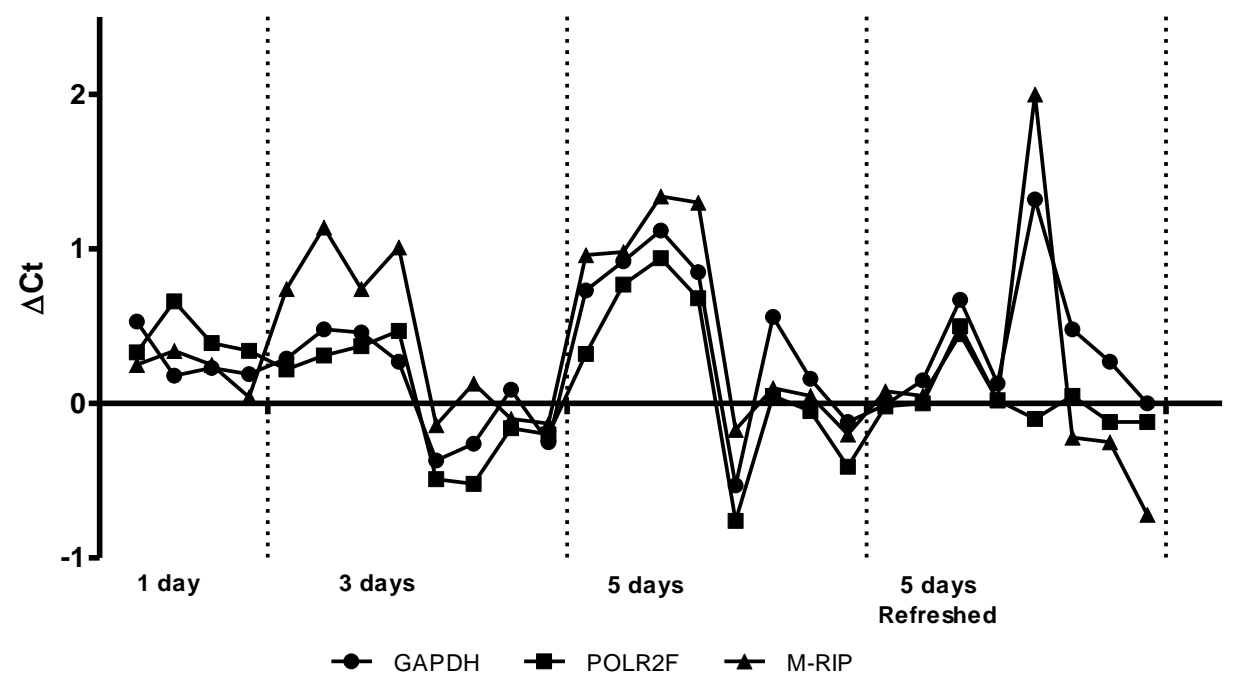

Figure 41b: Comparison of the expression of three reference genes in samples of SH-SY5Y cells treated with ethanol, and tobacco compounds used in this study. Each data point represents the difference in $\mathrm{Ct}$ between the treated sampled and an untreated SH-SY5Y control. 
The expression of POLR2F was not found to vary following exposure to ethanol, nicotine, HTPM, or QTPM. Cell samples from all treatment groups were tested to determine if treatment induced changes in the expression of these genes. The difference in $\mathrm{Ct}$ value between reference genes was compared for all three possible reference gene combinations, and no significant differences were observed (Fig. 41a). The Ct values for CDNA samples from treated cells were also normalized by subtracting the mean $\mathrm{Ct}$ value recorded in untreated samples. These values for POLR2F, GAPDH, and M-RIP were compared visually (Fig. 41b) and showed good general agreement. Trends in POLR2F and GAPDH expression levels were very closely matched, while M-RIP showed slightly greater variability. The slight deviations from the mean $\mathrm{Ct}$ of untreated samples are a reflection of the inherent variation in the instrument, pipettes and PCR enzyme activity.

Finally, Ct data for all three reference genes were analyzed with the geNorm v3.5 software. The geNorm software comprises a collection of VBA macros for Microsoft Excel that determines the most stable reference gene from a given set of tested candidate genes. It calculates an average stability variable $M$, by determining variation of the expression ratio for every pair of reference genes tested. Genes with the most stable expression have the lowest $M$ values. POLR2F was found to be the most stable reference gene, with an $M$ value of 0.025 ; while, GAPDH and M-RIP had $M$ values of 0.027 and 0.026 , respectively. Together, these tests confirm the stability of POLR2F in SH-SY5Y cells and validate the use of POLR2F as an appropriate reference gene for this study.

\subsection{2 - Effects of Ethanol Exposure on MAO-A \& MAO-B Gene Expression}

As described earlier, all TPM extracts used in this study were collected in absolute ethanol to retain as many of the volatile compounds as possible. This meant that cells treated with TPM were also exposed to $5.6 \mathrm{mM}$ ethanol during the exposure period. Ethanol has been reported to affect the gene expression of MAO-B in vitro (Ekblom et al., 1996). To control for any effects due to ethanol, the purified nicotine standard was reconstituted in ethanol, and vehicle controls treated with ethanol alone were prepared. The effect of ethanol exposure on the expression of MAO-A and MAO-B was then examined. 
(a)

MAO-A gene expression in SH-SY5Y cells exposed to ethanol

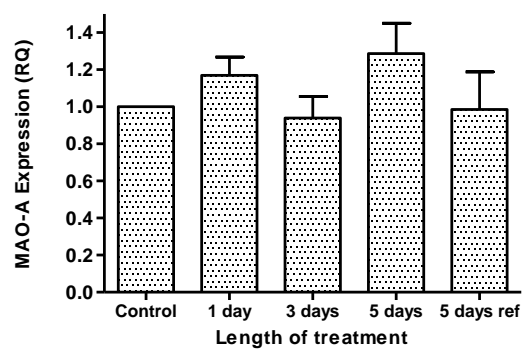

(b)

MAO-B gene expression in SH-SY5Ycells exposed to ethanol

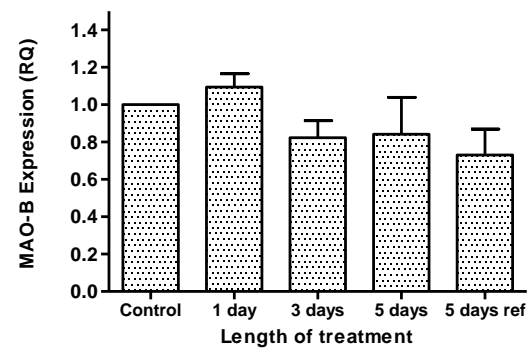

Figure 42: (a) MAO-A and (b) MAO-B gene expression in SH-SY5Y cells following treatment with 5.6 mM ethanol, expressed as mRNA expression relative to untreated control cells maintained over the same time period (RQ). No significant difference was observed in the expression of either gene following 1, 3, or 5 days of continuous ethanol treatment, or treatment under the 5 day refreshed regimen (5 days ref) (oneway ANOVA with Dunnett's post-test; $n \geq 3$ independent preparations for each condition).

Neither MAO-A nor MAO-B expression showed any statistically significant up- or downregulation in response to $5.6 \mathrm{mM}$ ethanol exposure for 1,3 , or 5 days continuously, or for 5 days under the refreshed treatment regimen (Fig. 42a-b, Table 14). While no statistically significant effects of the ethanol vehicle were observed, the effects of tobacco compounds on MAO-A and -B gene expression were normalized to these ethanol-treated controls.

Table 14: MAO-A and MAO-B gene expression in ethanol-treated SH-SY5Y cells

\begin{tabular}{lcccccc}
\hline Ethanol & \multicolumn{3}{c}{ MAO-A } & \multicolumn{3}{c}{ MAO-B } \\
Treatment & RQ & SEM & $n$ & RQ & SEM & $n$ \\
\hline 1 day & 1.17 & 0.09 & 3 & 1.09 & 0.07 & 7 \\
3 days & 0.94 & 0.11 & 4 & 0.82 & 0.09 & 6 \\
5 days & 1.28 & 0.16 & 8 & 0.84 & 0.19 & 7 \\
5 days refreshed & 0.99 & 0.20 & 6 & 0.73 & 0.14 & 6 \\
\hline
\end{tabular}

MAO-A and MAO-B expression levels are presented as mean values \pm SEM, relative to untreated controls for $n$ samples per group.

\subsection{3 - Effects of Tobacco Extract Exposure on MAO-A Gene Expression}

Gene expression of MAO-A was measured in SH-SY5Y cells treated with nicotine, Holiday ${ }^{\circledR}$ brand TPM (HTPM), and denicotinized Quest ${ }^{\circledR}$ brand TPM (QTPM) (Fig. 43). Cells treated with nicotine showed a slight, gradual increase in MAO-A mRNA expression over the time period 
tested. This increase was not statistically significant at one or three days, but a $26 \%$ increase reached significance after 5 days treatment with $0.2 \mu \mathrm{M}$ nicotine (two-way ANOVA, Bonferroni's multiple comparison post test), relative to the ethanol-treated control.

MAO-A gene expression in response to HTPM and QTPM exposure increased the 3-day time point by $23 \%$ and $35 \%$ respectively, although the result with HTPM was not significant. The increase due to QTPM exposure was statistically significant when tested with a two-way ANOVA test, but MAO-A gene expression returned to near-baseline levels after 5 days continuous treatment.

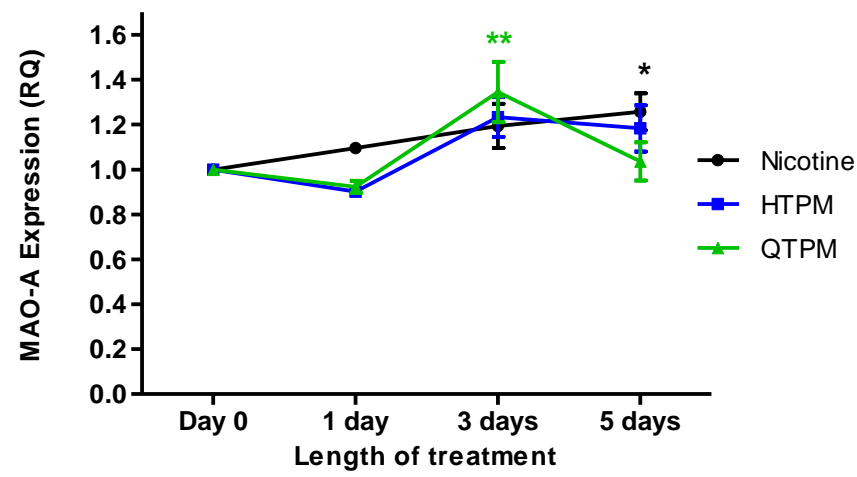

Figure 43: Changes in MAO-A gene expression in response to nicotine $(0.2 \mu \mathrm{M})$, HTPM $(0.03 \%)$, and QTPM (0.03\%) exposure are summarized above. MAO-A mRNA expression for treated cells was normalised to ethanol-treated controls maintained over the same time period. Results were tested by two-way ANOVA with Bonferroni's multiple comparison post-test $(\alpha=0.05) ; n \geq 3$ independent experiments for each condition (Table 15). ${ }^{*} \mathrm{P}<0.05 ; * \mathrm{P}<0.01$.

The drop in MAO-A expression at the 5-day time point might be due to metabolism of the active compounds by the cells. To test this, cultured cells treated for 5 days using the refreshed medium treatment regimen were also assayed for MAO-A gene expression. The results are presented with the data from the 1, 3, and 5 day experiments for comparison (Fig. $44 a-d)$

SH-SY5Y cells exposed to nicotine under the refreshed regimen showed a decrease in MAO-A gene expression to $78 \%$ of control (Fig. 44a). This was not significantly different from control expression, but was a significant reduction when compared to the expression in cells treated for 5 days continuously (one-way ANOVA, Bonferroni's multiple comparison post-test). In cells exposed to HTPM and QTPM extracts under this treatment regimen, MAO-A gene expression increased dramatically to approximately $380 \%$ and $360 \%$ of control cells, 
respectively. This increase was found to be highly significant $(p<0.001)$. It is notable that this increase in MAO-A gene expression was observed in cells treated with HTPM and QTPM, but not in cells treated with nicotine, suggesting that the non-nicotine components of cigarette smoke present in both HTPM and QTPM up-regulate expression of MAO-A after chronic exposure.

(a)

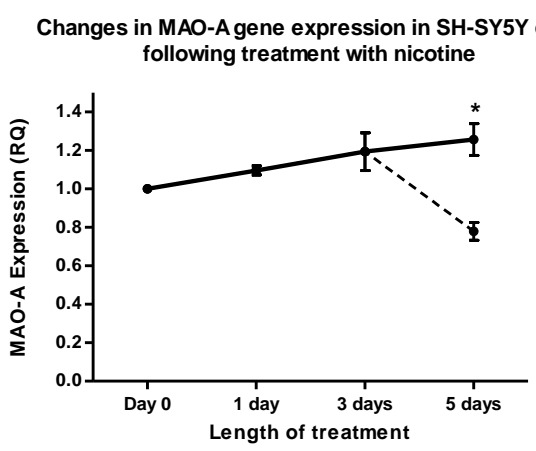

(c)

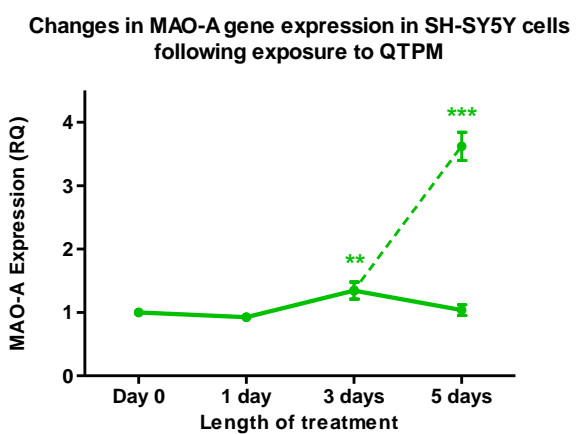

(b)

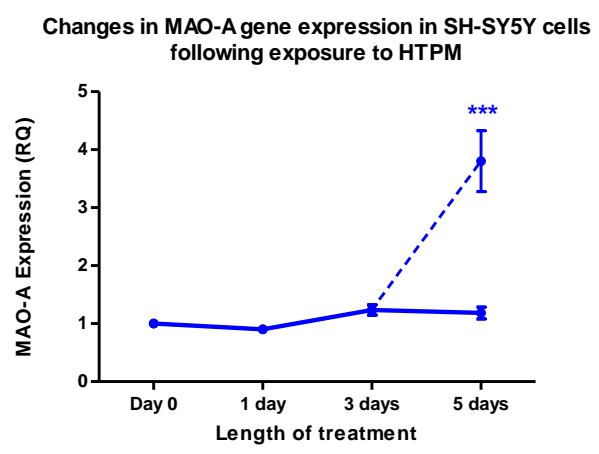

(d)

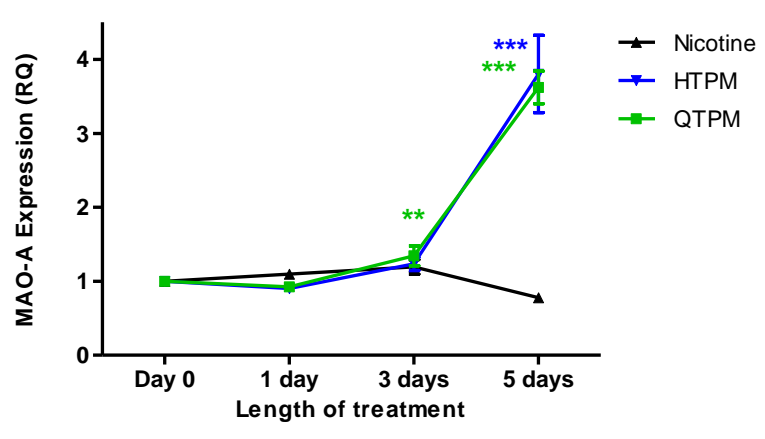

Figure 44: Changes in MAO-A gene expression following exposure to (a) nicotine, (b) HTPM, and (c) QTPM including the refreshed medium regimen. Solid lines connect results from Fig. 43 for cells treated for 1,3 , and 5 days continuously, and dashed lines represent cells treated with the refreshed regimen ( $\mathrm{n} \geq 3$ samples). Data for 1,3 , and 5 day refreshed regimens are re-presented in (d) for comparison. $* \mathrm{P}<0.05 ; * * \mathrm{P}<0.01 ; * * * \mathrm{P}<0.001$; two-way ANOVA with Bonferroni's multiple comparison post-test.

The increase in MAO-A gene expression in cells treated with HTPM and QTPM for the 5-day refreshed regimen was significantly increased above baseline MAO-A expression in control cells, and was also significantly different from cells treated for 5 days continuously (Fig. 45) $(P<0.001)$. Since both 5 -day refreshed tests showed significant changes in expression compared to the 5-day continuous treatment, it is possible that the active compounds in the tobacco smoke may be metabolized by the cells over longer exposures, as hypothesized. Changes in gene expression over all treatments and time periods are expressed in Table 15. 


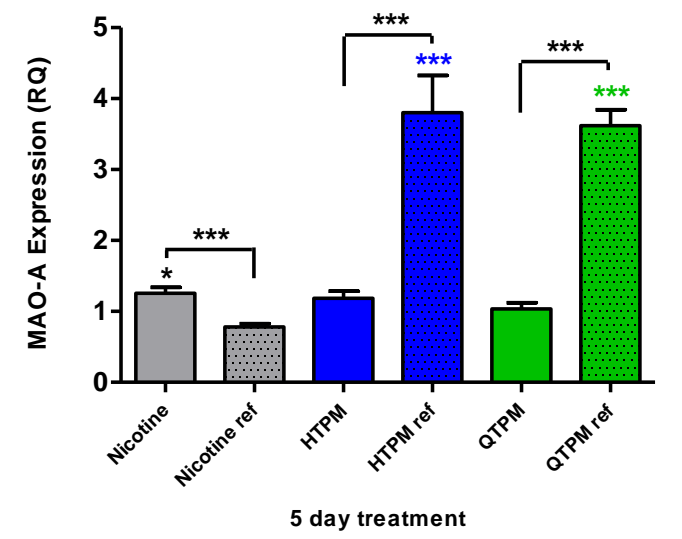

Figure 45: Comparison of MAO-A gene expression in SH-SY5Y cells treated with various tobacco compounds over 5 days ( $n \geq 12$ ), or 5 days with refreshed media on day $3(n=6)$, (from Fig. 44a-d). Increases in gene expression between 5 day treatment and the refreshed treatment were found to be highly significant (one-way ANOVA with Bonferroni's post test) for cells treated with nicotine, HTPM and QTPM. ${ }^{*} \mathrm{P}<0.05 ; * * \mathrm{P}<0.01 ; * * * \mathrm{P}<0.001$.

Table 15: Summary of changes in MAO-A gene expression in SH-SY5Y cells

\begin{tabular}{l|ccccccc|ccc}
\hline \multirow{2}{*}{ Treatment } & \multicolumn{3}{c}{ Nicotine } & \multicolumn{3}{c}{ HTPM } & \multicolumn{3}{c}{ QTPM } \\
& RQ & SEM & $n$ & RQ & SEM & $n$ & RQ & SEM & $n$ \\
\hline 1 day & 1.09 & 0.02 & 3 & 0.90 & 0.01 & 4 & 0.92 & 0.02 & 3 \\
3 days & 1.19 & 0.09 & 7 & 1.23 & 0.08 & 6 & $1.35^{* *}$ & 0.13 & 5 \\
5 days & $1.26 *$ & 0.08 & 13 & 1.18 & 0.10 & 12 & 1.04 & 0.08 & 14 \\
5 day refreshed & 0.78 & 0.04 & 6 & $3.80^{* * *}$ & 0.50 & 6 & $3.62^{* * *}$ & 0.22 & 6 \\
\hline
\end{tabular}

Summary of relative fold changes in MAO-A gene expression in SH-SY5Y cells treated with tobacco constituents. Sample sizes for each condition are expressed as $n$ different preparations. ${ }^{*} \mathrm{P}<0.05 ; * *$ $\mathrm{P}<0.01$; ** $\mathrm{P}<0.001$; two-way ANOVA with Bonferroni's multiple comparison post-test.

\subsection{4 - Effects of Tobacco Extract Exposure on MAO-B Gene Expression}

Nicotine exposure for 1,3 , or 5 days caused no significant or notable change in MAO-B gene expression compared to control cells (Fig. 46). Exposure to HTPM and QTPM tobacco extracts also induced no change in mRNA expression in SH-SY5Y cells. In cells exposed to HTPM, MAOB mRNA expression was increased by $29 \%$ after 5 days continuous treatment; however, this was not a statistically significant change. 


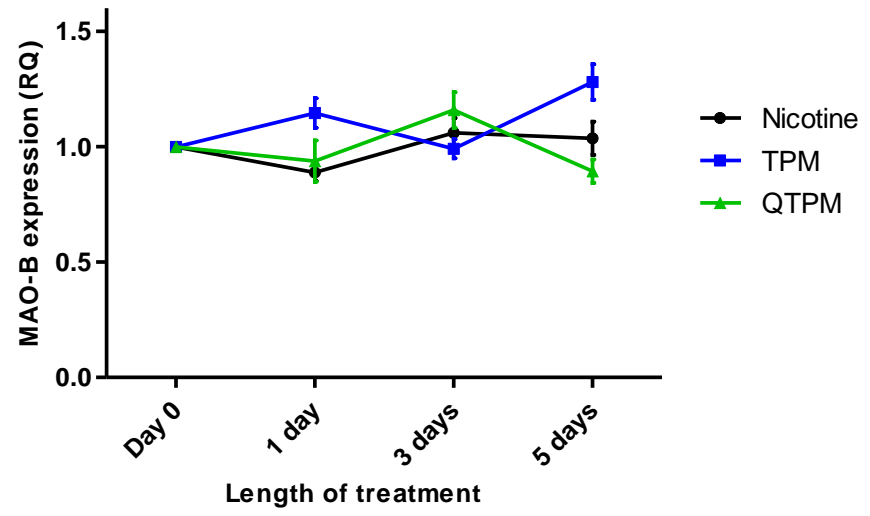

Figure 46: MAO-B mRNA levels measured by qRT-PCR in cells exposed to nicotine $(0.2 \mu \mathrm{M}), \mathrm{HTPM}$, and QTPM are compared. MAO-B mRNA expression for treated cells was normalised to ethanol-treated controls maintained over the same time period. No statistically significant results were observed in any treatment for 1,3 , or 5 days (two-way ANOVA with Bonferroni's post-test; $\alpha=0.05, n \geq 5$ independent preparations for each condition).

MAO-B gene expression was also examined in cells treated with nicotine, HTPM and QTPM for 5 days after refreshing the medium at day 3. When SH-SY5Y cells were exposed to nicotine under the refreshed medium regimen, mean MAO-B expression decreased slightly to $83 \%$ of baseline expression (Fig. 47a). This is similar to the effect on MAO-A expression. MAO-B mRNA expression increased significantly to $270 \%$ of baseline levels in cells treated with HTPM over the 5 day refreshed treatment (Fig. 47b). This increase in gene expression was highly significant (one-way ANOVA, Bonferroni's multiple comparison post-test). This effect was also observed in cells treated with QTPM for the refreshed regimen, which showed a $260 \%$ increase in expression (Fig. 47c). This pattern of MAO-B gene expression changes is similar to the changes observed in MAO-A expression when cells were treated with tobacco extracts using the same treatment regimen. This suggests that compounds within the tobacco extracts induce increases in MAO-B mRNA expression, but nicotine does not.

Since the 5-day refreshed medium exposure induced larger changes in MAO-B gene expression than the 5-day continuous treatment, this could suggest that the active components in TPM are lost after 3 days in culture. Changes in relative MAO-B gene expression following all treatments are summarized in Table 16. 
(a)

Changes in MAO-B gene expression in SH-SY5Ycells following exposure to nicotine

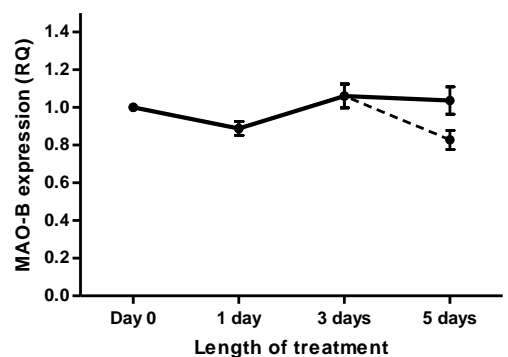

(c)

Changes in MAO-B gene expression in SH-SY5Ycells

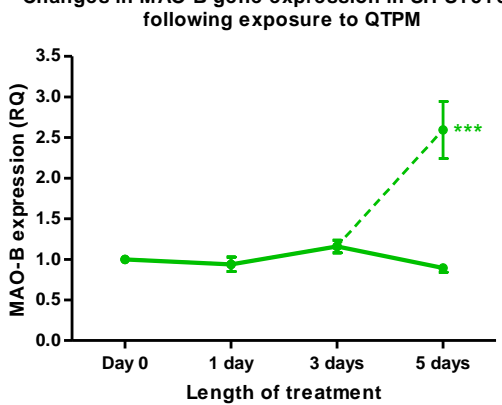

(b)

Changes in MAO-B gene expression in SH-SY5Ycells following exposure to HTPM

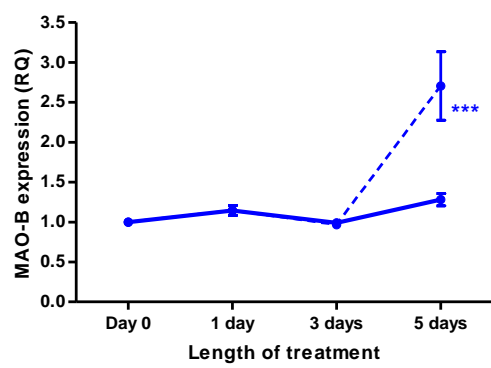

(d)

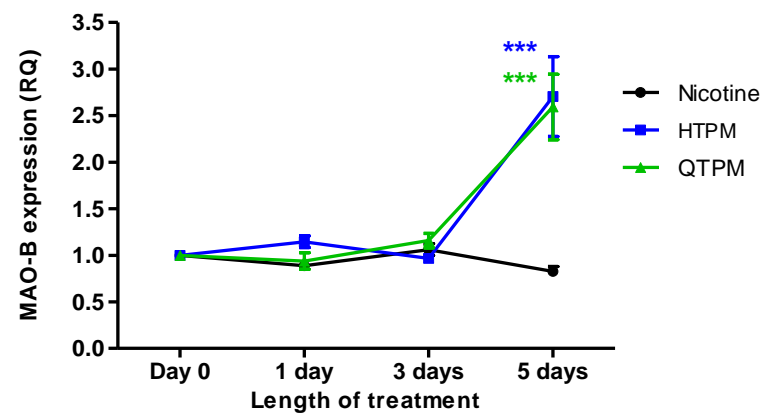

Figure 47: Changes in MAO-B gene expression following (a) nicotine, (b) HTPM, and (c) QTPM. Solid lines connect results from Fig. 46 for cells treated for 1, 3, and 5 days continuously, and dashed lines represent cells treated with the refreshed regimen. Data for 1, 3, and 5 day refreshed regimens are re-presented in (d) for comparison. Data were tested by two-way ANOVA with Bonferroni's multiple comparison test (*** $\mathrm{P}<0.001 ; \mathrm{n} \geq 5$ preparations for all conditions).

Table 16: Summary of changes in MAO-B gene expression in SH-SY5Y cells

\begin{tabular}{l|ccccccccccc}
\hline \multicolumn{1}{l}{ Treatment } & \multicolumn{3}{c}{ Nicotine } & \multicolumn{3}{c}{ HTPM } & \multicolumn{3}{c}{ QTPM } \\
& RQ & SEM & $n$ & RQ & SEM & $n$ & RQ & SEM & $n$ \\
\hline 1 day & 0.89 & 0.03 & 7 & 1.15 & 0.06 & 6 & 0.94 & 0.08 & 7 \\
3 days & 1.06 & 0.06 & 6 & 1.00 & 0.04 & 5 & 1.16 & 0.07 & 6 \\
5 days & 1.04 & 0.07 & 12 & 1.28 & 0.07 & 10 & 0.89 & 0.05 & 14 \\
5 day refreshed & 0.83 & 0.05 & 6 & $2.71 * * *$ & 0.42 & 6 & $2.59 * * *$ & 0.35 & 6
\end{tabular}

Summary of relative fold changes in MAO-B gene expression in SH-SY5Y cells treated with tobacco constituents. Sample size is expressed as $\mathrm{n}$ flasks prepared in independent experiments. *** $\mathrm{P}<0.001$ (one-way ANOVA with Bonferroni's post test;). 


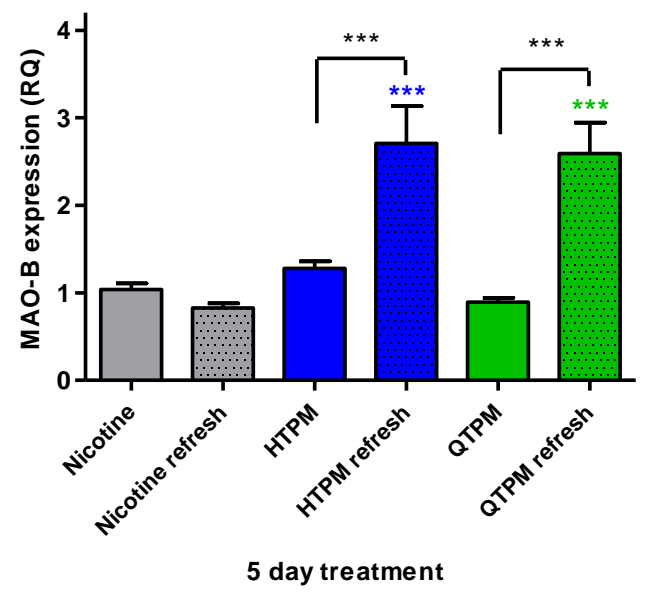

Figure 48: Comparison of MAO-B gene expression in SH-SY5Y cells treated with nicotine, HTPM, or QTPM for 5 days continuously, or 5 days with refreshed medium. Gene expression in cells treated with HTPM and QTPM with the refreshed regimen was significantly increased compared to untreated cells (one-way ANOVA with Bonferroni's post test; $\left.{ }^{* * *} \mathrm{P}<0.001\right)$.

\subsection{5 - Gene Expression in U-118 MG Cells Treated with Tobacco Compounds}

MAO-A and MAO-B mRNA was successfully amplified from U-118 MG cells, providing confirmation that this cell line expresses MAO-A and MAO-B. However both MAO-A and MAO-B were expressed at levels several fold lower in U-118 MG cells compared to SHSY5Y cells. Ct values were normalized to the expression of the reference gene, POLR2F, and expressed as $\Delta \mathrm{Ct}$ (Fig. 49).

(a)

MAO-A gene expression in SH-SY5Y and U-118 cells

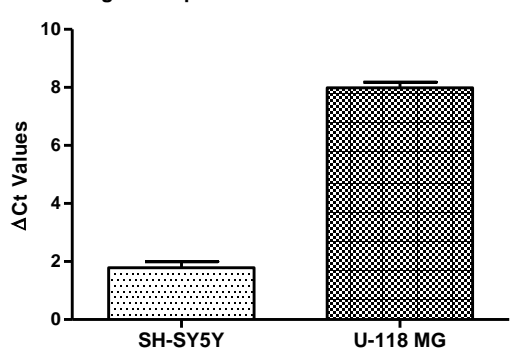

(b)

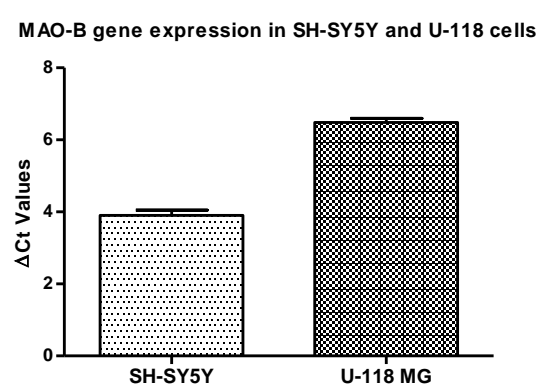

Figure 49: (a) MAO-A and (b) MAO-B gene expression in SH-SY5Y cells and U-118 MG cells relative to POLR2F reference gene expression, expressed as $\triangle \mathrm{Ct}$. Both genes were expressed at significantly lower levels (higher $\mathrm{Ct}$ ) in $\mathrm{U}-118 \mathrm{MG}$ cells compared to SH-SY5Y cells (Student's two-tailed t-test, $\mathrm{P}<0.0001, \mathrm{n} \geq 8$ independent experiments). 
Gene expression was examined in U-118 MG cells to determine if the changes in MAO genes observed in SH-SY5Y cells exposed to tobacco compounds were also present in a glial cell model. MAO-A and -B expression was measured in U-118 MG cells exposed to nicotine, HTPM, and QTPM for 5 days continuously. Only one time point was chosen for this experiment, the continuous 5-day regimen, as this was the longest exposure, and it was expected it would show the greatest response to treatment. These experiments were carried out prior to discovery that refreshing the medium at day 3 altered the 5 -day response.

Expression of MAO-A and MAO-B after exposure to ethanol was not significantly different in U-118 MG glioma cells compared to untreated controls, indicating that ethanol did not affect expression of these genes in U-118 MG (Fig. 50). The percent change in MAO-A and MAO-B gene expression in U-118 MG cells relative to controls was also no different from the percent change in expression in SH-SY5Y neuroblastoma cells relative to control (Figs. 50a and 50b). This indicates that the U-118 MG cell line responds in a similar manner to SH-SY5Y when exposed to ethanol, despite having a lower overall mRNA expression than seen in SH-SY5Y cells. Although ethanol did not induce any significant change in MAO-A or MAO-B gene expression in U-118 MG cells, gene expression data following exposure to nicotine, HTPM, and QTPM were normalized to ethanol-treated cells to control for any effects of vehicle.

(a) MAO-A gene expression in SH-SY5Y and U-118 MG cells
exposed to ethanol

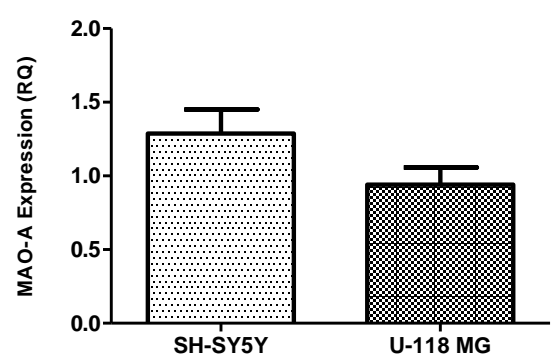

(b)
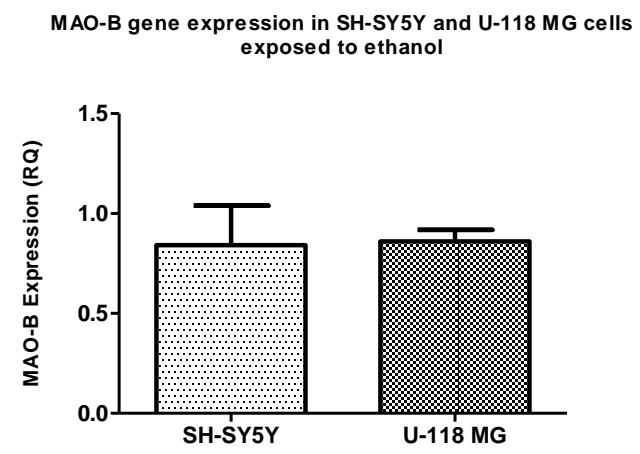

Figure 50: Relative quantification of (a) MAO-A and (b) MAO-B gene expression in SH-SY5Y cells and U-118 MG cells following 5 days continuous exposure to ethanol $(5.6 \mathrm{mM})$. No significant differences in the expression of either gene were observed in U-118 MG cells relative to untreated cells, or compared to SH-SY5Y cells (one-way ANOVA with Dunnett's multiple comparison test; $\alpha=0.05 ; n=8$ independent preparations).

MAO-A gene expression in U-118 MG cells did not vary significantly from control expression after 5 days continuous treatment with nicotine or QTPM (Fig. 51a). As 
reported above (section 6.3.3), a modest increase in MAO-A mRNA expression was observed in SH-SY5Y cells receiving nicotine treatment for 5 days, but this effect was not recorded in U-118 MG cells under the same regimen. U-118 MG cells treated with HTPM showed an increase in MAO-A expression to $133 \%$ of control $(P=0.01)$. This percent change in expression was not significantly different to the percent change in expression of MAO-A after 5 days seen in SH-SY5Y cells. Similarly, there was no difference in relative MAO-A expression between U-118 MG cells and SH-SY5Y cells in any other treatment group.

Treatment with tobacco compounds did not alter MAO-B expression in U-118 MG cells after 5 days exposure (Fix. 51b). No difference was observed in the relative MAO-B expression level between SH-SY5Y and U-118 MG cells treated with nicotine or QTPM; however, the difference in expression between the two cell lines treated with HTPM was found to be significant $(P<0.001)$. Neither the increase seen in $\mathrm{SH}-\mathrm{SY} 5 \mathrm{Y}$ cells nor the decrease in U-118 MG cells was found to differ significantly from the respective vehicletreated controls. The difference between the two cell lines was unexpected, but may suggest subtle differences in the response of these two cell lines to tobacco exposure. The changes in MAO-A and $-\mathrm{B}$ gene expression in $\mathrm{U}-118$ cells following 5 days continuous treatment are summarized and compared to SH-SY5Y responses in Table 17.

(a)

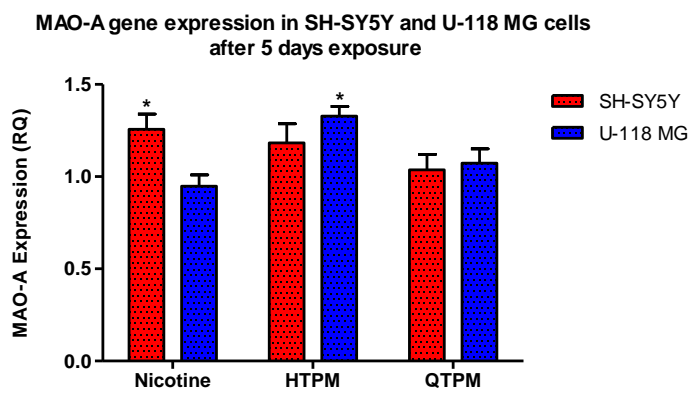

(b)

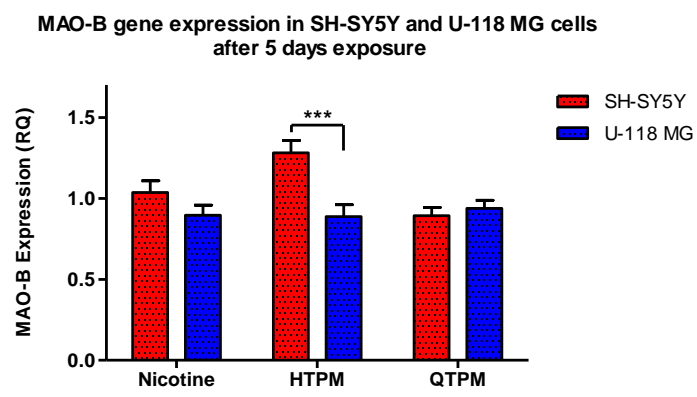

Figure 51: Relative quantification of MAO-A (a) and MAO-B (b) gene expression in SH-SY5Y cells and U-118 MG cells following 5-day continuous exposures to tobacco compounds. SH-SY5Y data are taken from Figs. 43 \& 46. Small but significant increases in MAO-A expression were observed in U-118 cells receiving HTPM treatment. No other significant differences were observed between MAO-A or MAO-B gene expression in $\mathrm{U}-118$ cells treated with any compound relative to vehicle-treated $\mathrm{U}-118$ cells. Gene expression changes of MAO-A in U-118 cells did not differ from the gene expression changes measured in SH-SY5Y cells for any treatment group; however, there was a significant difference in the percent change in MAO-B gene expression in U-118 cells treated with HTPM compared to that in SH-SY5Y cells. (Two way ANOVA with Bonferroni's post test. $\left.{ }^{*} \mathrm{P}<0.05 ; * * * \mathrm{P}<0.001\right)$ 
Table 17: Comparison of changes in MAO-A \& MAO-B gene expression in U-118 MG and SH-SY5Y cells

\begin{tabular}{|c|c|c|c|c|c|c|c|c|c|c|c|c|}
\hline & \multicolumn{6}{|c|}{ MAO-A } & \multicolumn{6}{|c|}{ MAO-B } \\
\hline & \multicolumn{2}{|c|}{ SH-SY5Y } & & \multicolumn{3}{|c|}{ U-118 } & \multicolumn{2}{|c|}{ SH-SY5Y } & & \multicolumn{3}{|c|}{ U-118 } \\
\hline & $\mathrm{RQ}$ & SEM & $n$ & $\mathrm{RQ}$ & SEM & $n$ & $\mathrm{RQ}$ & SEM & $n$ & $\mathrm{RQ}$ & SEM & $n$ \\
\hline Nicotine & $1.26 *$ & 0.08 & 13 & 0.95 & 0.06 & 8 & 1.04 & 0.07 & 12 & 0.90 & 0.06 & 8 \\
\hline HTPM & 1.18 & 0.10 & 12 & $1.33^{*}$ & 0.05 & 6 & 1.28 & 0.08 & 10 & 0.89 & 0.07 & 8 \\
\hline QTPM & 1.04 & 0.08 & 14 & 1.07 & 0.08 & 7 & 0.89 & 0.05 & 14 & 0.93 & 0.05 & 8 \\
\hline
\end{tabular}

Summary of relative fold changes in MAO-A and MAO-B gene expression in U-118 MG cells treated for 5 days. Data from SH-SY5Y cells treated using the same regimen (Tables $15 \& 16$ ) are also reported here for comparison. Sample sizes are expressed as independent preparations $(n)$.

\subsection{6 - Mecamylamine Treatment}

Cultured SH-SY5Y cells were treated with nicotine, HTPM and QTPM in the presence of 10 $\mu \mathrm{M}$ mecamylamine, a non-specific $\mathrm{nAChR}$ antagonist, to determine if the changes in MAO-A gene expression in response to tobacco extracts were dependent on $\mathrm{nAChR}$ activity. Only MAO-A, and not MAO-B, gene expression was examined in conjunction with mecamylamine because experiments using the continuous treatment regimen showed MAO-B expression did not change following nicotine, HTPM, or QTPM exposure (Fig. 46). These experiments were carried out prior to discovery that replacing the medium at day 3 changed the 5 -day response.

\subsubsection{1 - Effects of Mecamylamine}

To determine if mecamylamine affected MAO-A gene expression, cells exposed to mecamylamine alone, or mecamylamine in the presence of ethanol were assayed for MAO-A gene expression by qRT-PCR. Mecamylamine alone increased MAO-A gene expression by $210 \%$ compared to untreated SH-SY5Y controls (Fig. 52). When cells were treated with mecamylamine in the presence of ethanol, MAO-A gene expression was increased further, by $310 \%$ of expression in untreated controls. This effect on MAO-A gene expression was unexpected, and therefore all cells treated in conjunction with mecamylamine were normalized to the mecamylamine \& ethanol-treated control to correct for this effect. 


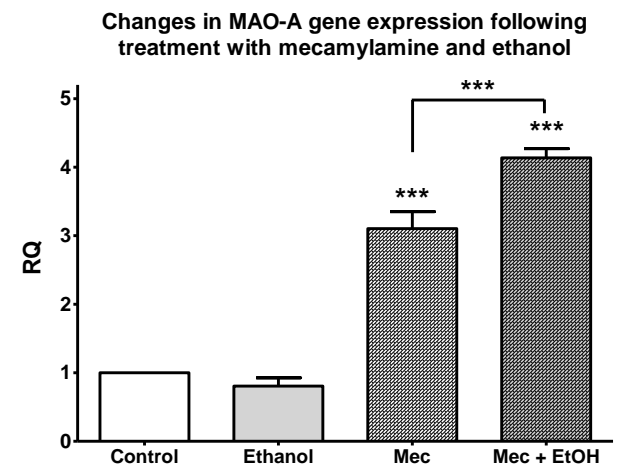

Figure 52: Comparison of MAO-A gene expression in $\mathrm{SH}-\mathrm{SY} 5 \mathrm{Y}$ cells receiving treatment with ethanol and/or mecamylamine. Ethanol $(5.6 \mathrm{mM})$ treatment had no effect on MAO-A expression, but treatment with $10 \mu \mathrm{M}$ mecamylamine, or mecamylamine and ethanol together caused significant up-regulation of MAO-A (one-way ANOVA with Bonferroni's multiple comparison test, $\mathrm{P}<0.0001 ; \mathrm{n}$ $\geq 6$ preparations in each condition). $* * * \mathrm{P}<0.001$.

It was expected that the increases seen in MAO-A gene expression in response to tobacco extract would be reversed when treated with mecamylamine if these increases were dependent on $\mathrm{nAChR}$ activation. The net effect of this would be that recorded mRNA expression in nicotine, HTPM, and QTPM treatment groups would fall toward levels seen in vehicle controls, effectively expressed as a relative quantitation of approximately 1.0. Cells treated with nicotine appeared to obey this trend (Fig. 53).

MAO-A gene expression in SH-SY5Y cells treated with tobacco compounds and mecamylamine

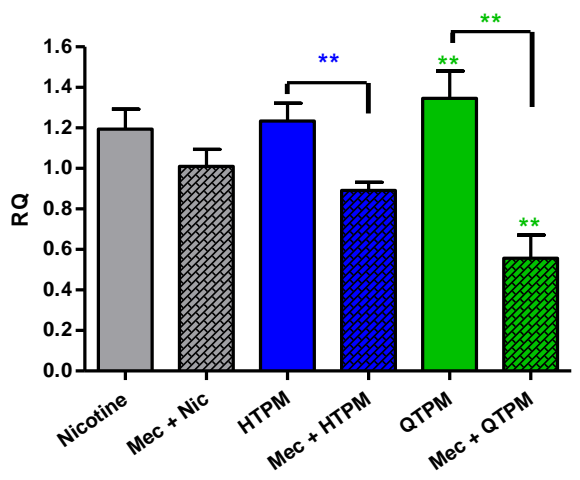

Figure 53: Comparison of MAO-A gene expression in SH-SY5Y cells treated for 3 days with nicotine, HTPM, and QTPM alone, or in conjunction with $10 \mu \mathrm{M}$ mecamylamine (Mec). A significant inhibition of MAO-A mRNA expression was observed between HTPM-treated and HTPM with mecamylamine-treated cells, and also between QTPM-treated and QTPM plus mecamylaminetreated cells. $* * \quad \mathrm{P}<0.01$. Expression in cells treated with nicotine, HTPM, and QTPM was normalised to expression in ethanol-treated ccontrols, and expression in cells receiving mecamylamine in conjunction with tobacco compounds was normalised to expression in controls treated with ethanol and mecamylamine. 
Nicotine treatment for 3 days appeared to increase MAO-A mRNA expression slightly to a mean RQ of 1.19 , although this was not statistically significant. When cells were treated with nicotine in conjunction with mecamylamine mean expression decreased to 1.01 . Although this change fits the predicted trend, it was not significant (Student's two-tailed t-test, $p=0.19$ ). In contrast, HTPM-treated cells showed a non-significant $23 \%$ increase in expression relative to vehicle controls, which was significantly inhibited by mecamylamine (Student's two-tailed t-test, $p=0.005$ ). Despite lacking nicotine, this same trend was observed in cells treated with QTPM, which showed a significant $35 \%$ increase in MAO-A mRNA expression that was significantly reduced to $56 \%$ of control expression by mecamylamine (Student's two-tailed t-test, $p=0.0015$ ).

Overall, these results suggest that the increases in MAO-A gene expression in SH-SY5Y cells treated with tobacco compounds are linked to activation of nAChRs, as these changes are reversible in the presence of a nAChR inhibitor. Changes in gene expression following treatment with mecamylamine are summarized in Table 18.

Table 18: MAO-A expression in SH-SY5Y cells treated with tobacco compounds and mecamylamine.

\begin{tabular}{l|ccc|ccc}
\hline \multicolumn{1}{c}{ Treatment } & \multicolumn{1}{c}{ Plus Mecamylamine } \\
& RQ & SEM & $n$ & RQ & SEM & $n$ \\
\hline Nicotine & 1.19 & 0.09 & 7 & 1.01 & 0.08 & 6 \\
HTPM & 1.23 & 0.09 & 6 & 0.89 & 0.04 & 6 \\
QTPM & $1.35^{* *}$ & 0.13 & 5 & 0.56 & 0.11 & 6 \\
\hline
\end{tabular}

Summary of changes in MAO-A expression in SH-SY5Y cells treated with tobacco compounds in the presence or absence of mecamylamine. Sample sizes are expressed as $n$ preparations (see Fig. 53). ** $\mathrm{P}<0.01$.

\subsubsection{2 - Validation of Mecamylamine Assay}

Given that mecamylamine inhibited responses to QTPM with no nicotine present, it was felt that the action of the inhibitor should be validated. To determine if mecamylamine was behaving as predicted in inhibiting nAChRs, the expression of elF4A2 mRNA was examined. The expression of this gene has been reported to increase in response to nicotine treatment, and has been demonstrated to be dependent on the activation of nAChRs (Dunckley \& Lukas, 2006). 
The expression of elF4A2 mRNA was decreased in cells treated with ethanol and mecamylamine together relative to untreated SH-SY5Y cells (Fig. 54a) (one-way ANOVA with Dunnett's multiple comparison test, $p<0.05)$. Treatment with ethanol alone reduced elF4A2 expression even more to $40 \%$ of untreated cells. To correct for these effects, gene expression data for cells treated with nicotine or nicotine plus mecamylamine were normalized to the ethanol-treated and mecamylamine plus ethanol-treated cells, respectively.

In response to treatment with nicotine, SH-SY5Y cells showed a significant $81 \%$ increase in elF4A2 expression relative to vehicle controls (Fig. 54b). Addition of mecamylamine significantly reduced elF4A2 expression to that of controls (one-way ANOVA with Bonferroni's multiple comparison test, $\mathrm{P}<0.01$ ). These results are in agreement with the previous study by Dunckley \& Lukas (2006), and demonstrate that mecamylamine is performing as expected.

(a)

Expression of eIF4A2 in SH-SY5Y cells after treatment with ethanol and mecamylamine for 3 days

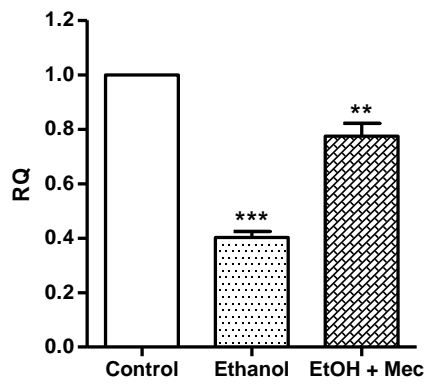

(b)

Expression of elF4A2 in SH-SY5Y cells treated with nicotine and mecamylamine for 3 days

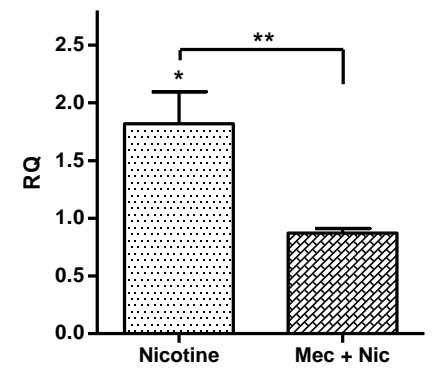

Figure 54: (a) Expression of elF4A2 mRNA in SH-SY5Y treated for 3 days with ethanol $(5.6 \mathrm{mM})$ or mecamylamine ( $\mathrm{Mec}, 10 \mu \mathrm{M})$. Gene expression was significantly down-regulated after both treatments (one-way ANOVA with Dunnett's multiple comparison test, $p<0.05$ ). (b) elF4A2 expression in cells treated with nicotine, or nicotine in the presence of mecamylamine. Nicotine treatment alone caused up-regulation of elF4A2 as expected, and this increase was blocked by mecamylamine (one-way ANOVA with Bonferroni's multiple comparison test, $\mathrm{P}<0.05, \mathrm{n}=3$ for all conditions. $\left.{ }^{*} \mathrm{P}<0.05 ;{ }^{*} \mathrm{P}<0.01 ; * * * \mathrm{P}<0.001\right)$.

\subsection{7-Western Blotting}

Western blotting analysis was performed on the same samples prepared for RT-PCR to determine if changes in gene expression were translated into changes in protein abundance. The 5-day refreshed media samples were chosen for these experiments, as they 
demonstrated the greatest increases in gene expression in response to treatment with tobacco extracts (refer to sections 6.3.3 and 6.3.4).

Protein abundance was quantified by densitometry of the immuno-specific bands identified on the Western blots (Fig. 55a \& b). As there was significant variation in the data, raw density figures were normalized to the density of a $\beta$-tubulin house-keeper band, and then expressed as percent of control. Ethanol-treated cells were used as the control. No significant or notable changes in MAO-A or MAO-B protein expression were seen following treatment with any of the tobacco extracts. Treatment with QTPM appeared to cause a decrease in MAO protein abundance to $70 \%$ of control, but this decrease was not significant. Thus, although substantial increases in MAO-A and MAO-B gene expression were observed in cells treated with HTPM and QTPM, no corresponding increase in MAO-A and MAO-B protein was evident.

(a)

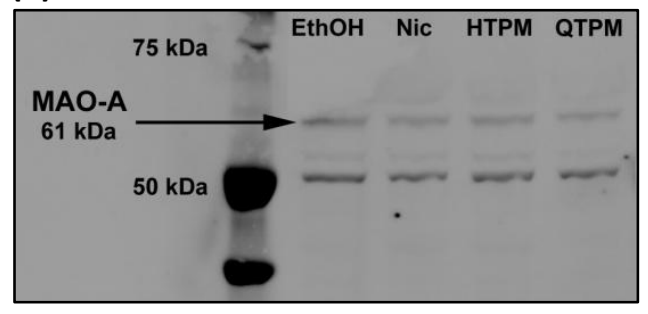

(c)

Relative band densities on MAO-A Western blots

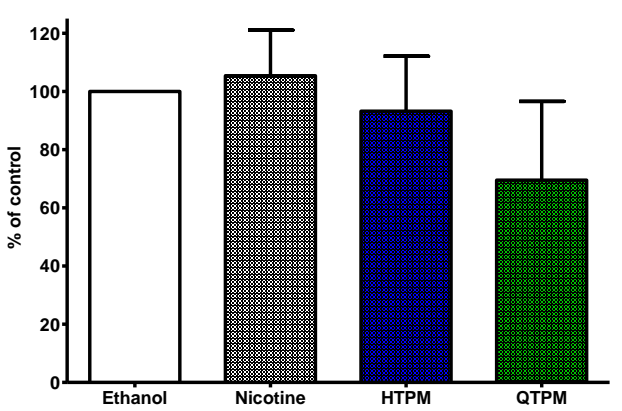

(b)

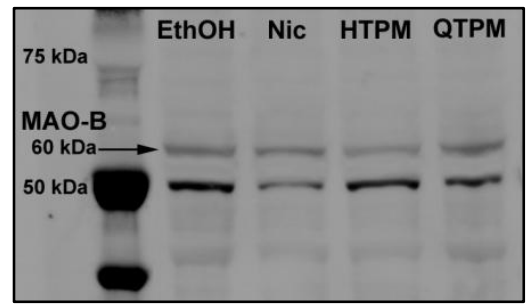

(d)

Relative band densities on MAO-B Western blots

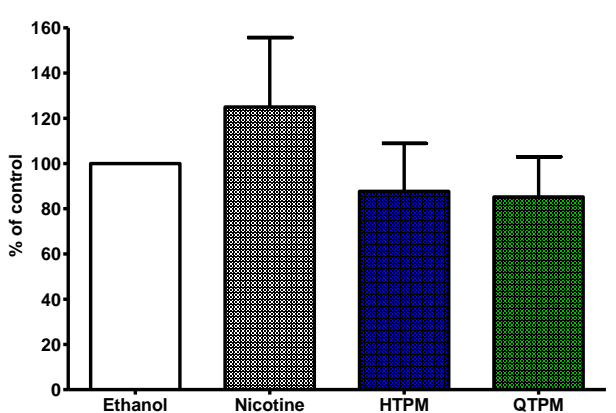

Figure 55: Representative Western blots of (a) MAO-A and (b) MAO-B in SH-SY5Y cells treated for 5 days with ethanol, nicotine, HTPM, or QTPM, and receiving refreshed medium on day 3. MAO-A and MAO-B specific bands are labeled at 61 and $60 \mathrm{kDa}$ respectively, and the $\beta$-tubulin loading control is evident at $50 \mathrm{kDa}$. Relative electrophoretic band densities of MAO-A immuno-specific bands (c) and MAO-B immuno-specific bands (d) are expressed here as percent of the ethanol control (mean + SEM). No notable differences were observed in protein abundance on Western blots for MAO-A or MAO-B (one-way ANOVA with Bonferroni's post-test; $\alpha=0.05 ; n=4$ blots from 4 independent preparations). 


\section{4-DISCUSSION}

\subsection{1 - Effects of Ethanol on MAO-A and MAO-B Expression}

Ethanol had no significant effect on the gene expression of MAO-A or MAO-B in SH-SY5Y cells at any of the time points tested in this study. This indicates that the ethanol vehicle used to solubilize the TPM samples does not interfere with the expression of MAO-A or MAO-B at the concentrations tested. A previous study by Ekblom et al. (1996) reported that MAO-B gene expression was significantly up-regulated in SH-SY5Y cells treated with ethanol for 24 hours. However, Ekblom et al. used ethanol concentrations of $50 \mathrm{mM}$ and $100 \mathrm{mM}$ which are sufficient to cause intoxication in humans, but the present study used considerably lower concentrations of ethanol $(5.6 \mathrm{mM})$. Thus, the concentration of ethanol used in the present study was too low to up-regulate MAO-B gene transcription as previously reported by Ekblom et al.

\subsection{2 - Changes in MAO-A and -B Expression Following Exposure to Tobacco}

\section{Compounds}

Although many studies have examined the effects of tobacco and tobacco smoke on the enzyme activity of MAO-A and MAO-B, the present study is the first to investigate changes in the expression of the monoamine oxidase genes. Because nicotine, HTPM, and QTPM inhibited total MAO activity in SH-SY5Y cells after 5 days continuous exposure (Section 5.3.3) it was thought these compounds may alter the expression of the MAO-A or MAO-B genes.

Cells treated with nicotine showed a gradual increase in MAO-A gene expression over the time period tested, up to 5 days continuous treatment when this up-regulation reached statistical significance. However, MAO-A up-regulation was not observed in cells treated with nicotine for 5 days using the refreshed medium regimen. This result is unusual, since if nicotine caused the up-regulation in MAO-A gene expression after 5 days continuous treatment, it should also induce up-regulation following the refreshed regimen. Taken together, these results suggest that cellular metabolites that accumulate as a result of the longer incubations without refreshing the culture medium may be increasing the expression of MAO-A mRNA. However, if this is the case all 5-day incubations should also induce up-regulation of MAO-A, though this was not observed. 
No consistent changes in MAO-A gene expression were observed in cells treated with HTPM or QTPM for up to 5 days of continuous exposure, although QTPM increased gene expression at 3 days, but this fell back to baseline levels after 5 days. In contrast, treatment with both of these tobacco smoke extracts using the refreshed medium regimen significantly increased the expression of MAO-A to more than three times the expression of untreated cells. Similarly, although these TPM samples had no effect on the gene expression of MAO-B after up to 5 days continuous treatment, exposure using the refreshed regimen caused a significant increase in MAO-B mRNA expression. In both cases, there was no difference in the upregulation induced by either HTPM, or QTPM, implying non-nicotine components of tobacco smoke are responsible for this effect. Since tobacco extracts are known to inhibit MAO-A and MAO-B enzyme activity, this increase in mRNA expression could indicate the cells are upregulating transcription of these genes to compensate for the enzymatic inhibition in an attempt to maintain a homeostatic level of MAO activity. The present study found an increase in total MAO enzyme activity in SH-SY5Y cells following exposure to HTPM and QTPM using the refreshed medium regimen (Section 5.3.3) (although the increase was significant for HTPM only), which is in agreement with this hypothesis. However, Western blotting analysis showed no significant increase in the amount of MAO-A or -B protein. This may indicate that MAO mRNA is being degraded before translation, or that tobacco smoke extracts cause an increase in the turn-over of MAO-A and MAO-B proteins. Additionally, this lack of correlation between mRNA transcription and protein abundance is not uncommon, and many studies examining individual gene and gene product data find only a weak, or no correlation (Greenbaum et al., 2002). Several reasons have been proposed for this, including differences in the half life of the protein in vivo, translational regulation, and a significant amount of experimental error and variation with respect to different conditions and techniques (Nie et al., 2006). This last factor may be applicable to the present study, as protein abundance was assessed by Western blotting, which is considered a semi-quantitative technique.

Since the present study is the first to examine MAO-A and MAO-B gene expression in response to nicotine and tobacco smoke extracts, there is currently little information available to corroborate the findings and put them in context to tobacco addiction. However interestingly, a recent study has proposed that tobacco exposure induces increases in MAO-A and MAO-B gene expression through epigenetic regulation of the gene promoter (Launay et al., 2009). Their study investigated MAO-B activity in the platelets of dependent smokers and found that former smokers had a significantly greater platelet MAO-B activity than either current smokers or subjects who had never smoked. Upon further investigation this was attributed to 
higher concentrations of MAO enzyme in the platelets of former smokers. Their study found that both smokers and former smokers had increased platelet MAO-B concentrations implying that smoking up-regulated MAO-B and this increased expression persisted long after quitting smoking, suggesting a smoking-induced modification of the MAO-B gene. The MAO-B core promoter contains a number of $\mathrm{CpG}$ sites that can be methylated, which reduces transcription of the MAO-B gene, and further genetic analysis showed never-smokers had a much higher methylation frequency at each of the $\mathrm{CpG}$ sites in the promoter. They verified that tobacco smoke was responsible for these changes in gene methylation, demonstrating that nucleic acid demethylase activity was significantly higher in lung preparations of mice exposed to tobacco smoke. Moreover, they also demonstrated that nucleic acid demethylase activity was increased by treatment with harman and nor-harman, two known MAO inhibitors present in tobacco smoke. To summarize, the findings of Launay et al. (2009) strongly suggest that exposure to tobacco smoke reduces methylation of the MAO-B promoter through increased activity of nucleic acid methyltransferase, resulting in up-regulation of MAO-B gene expression. This is manifested as an increase in platelet MAO-B protein which endures after tobacco smoke exposure is ceased. Overall platelet MAO activity in current smokers remains inhibited due to the ongoing exposure to MAO inhibitors in tobacco smoke, while MAO activity in former smokers is higher as they are no longer exposed to these inhibitors.

An earlier study by Pinsonneault et al. (2006) reported that the MAO-A gene was also subject to significant methylation at CpG sites in the promoter region. In a complex study investigating the epigenetic and genetic factors associated with MAO-A gene regulation in human brain samples, they found that cis-acting factors had a strong influence on mRNA expression. Female samples showed extensive and variable methylation of $\mathrm{CpG}$ sites, but the degree of methylation was not correlated with mRNA expression. Genetic polymorphisms were also found to influence MAO-A mRNA expression, but could not completely account for regulation of the gene. Pinsonneault et al. (2006) concluded that both genetic and epigentic factors contributed in a similar manner to MAO-A mRNA expression in females, and proposed that MAO-A promoter methylation modulates transcription of the gene. Ineterestingly, this study reported male samples showed no significant methylation of the MAO-A gene locus. It is unknown how these differences in promoter methylation may affect protein expression or monoamine metabolism in vivo (Pinsonneault et al., 2006).

The findings of Launay et al. (2009) are relevant to the present study, since the present study found exposure to tobacco smoke extracts induced an increase in total MAO enzyme activity 
(section 5.3.3), and up-regulation of MAO-A and MAO-B gene expression (Fig. 44d \& 47d) in SH-SY5Y cells. Further investigation is warranted to determine if these changes in MAO activity and expression in SH-SY5Y cells also result from demethylation of the promoter regions by increased nucleic acid demethylase activity.

However, as discussed previously, cell culture conditions are a significant factor in the behavior of the cells used in this study. Although flasks of cells treated for 3 and 5 days continuously had not reached complete confluence it is likely they may have suffered from nutrient depletion or negative effects from cellular metabolites as a result of the longer exposure periods. To avoid these effects it would be preferable to culture cells using a continuous perfusion culture system, as mentioned in section 5.3.3 of this thesis, or to change the medium daily. Since this perfusion system was not available to the experimenter, the refreshed medium treatment regimen was employed in an attempt to counter some of the effects of longer cell exposures.

\subsection{3 - Effects of Mecamylamine Treatment on MAO-A Gene Expression}

Initially, gene expression was measured for SH-SY5Y cells treated with tobacco compounds for 1,3 , or 5 days continuously, and the results from those experiments informed the experimental design for the mecamylamine experiments. As discussed above, MAO-A gene expression increased significantly in cells treated with QTPM for three days, but no change was observed in cells treated with nicotine or tobacco smoke extract. This questioned the role of nicotine in the MAO-A response, since QTPM lacks nicotine. To identify whether $\mathrm{nAChR}$ activation was required to induce changes in MAO gene expression, cells were incubated with tobacco compounds in the presence of mecamylamine for three days, and only MAO-A gene expression was assayed. If MAO-A gene expression was regulated through a nAChR-dependant signaling mechanism, then treatment with the nAChR antagonist mecamylamine should reverse any changes in MAO-A expression observed.

When SH-SY5Y cells were treated with mecamylamine MAO-A mRNA expression was significantly up-regulated compared to untreated controls. This result was unexpected, and is difficult to explain, since no direct interaction is known between mecamylamine and MAO-A. In their earlier study, Dunckley \& Lukas (2006) reported that mecamylamine altered the expression of a number of genes in SH-SY5Y, causing effects similar to those of nicotine alone. While it is possible that mecamylamine may act via a secondary non-nAChR path to indirectly 
affect the expression of a number of genes, these findings also suggest regulation of MAO-A gene expression in SH-SY5Y cells is dependent on an endogenous cholinergic signaling mechanism that is inhibited by mecamylamine exposure. While this may be the case in vivo, it is not certain whether SH-SY5Y cells exhibit this endogenous signaling (Dunckley \& Lukas, 2006).

In cells treated for 3 days continuously, MAO-A gene expression seemed to increase slightly with exposure to nicotine and HTPM, and more so with QTPM exposure. However, when mecamylamine was added, all of these increases were abolished. These results imply that inhibition of nAChRs has reversed the tobacco smoke-induced increases in MAO-A expression, suggesting that tobacco smoke extracts influence regulation of MAO-A gene expression through a nAChR-dependent mechanism. This finding is significant, since this response was recorded in the nicotine-free QTPM-treated cells, indicating that non-nicotine components of tobacco smoke can induce significant cellular effects through activation of nAChRs. However, the results of this experiment should be interpreted with caution, since the origin of mecamylamine's effect on MAO-A expression is not known.

Furthermore, this experiment examined the effect of mecamylamine on gene expression changes after 3 days continuous treatment with tobacco compounds. However, subsequent research found mRNA expression of both MAO-A and MAO-B was more profoundly affected when cells were treated with nicotine and tobacco smoke extracts using the 5-day refreshed medium regimen. It would be interesting to test the effects of mecamylamine on the significant up-regulation of MAO-A and MAO-B observed in cells treated with nicotine, HTPM, and QTPM using this exposure regimen. If indeed mecamylamine does block up-regulation in cells receiving tobacco extracts in the refreshed medium regimen, this would add further weight to the tentative conclusion that MAO-A is up-regulated by a nAChR-dependent mechanism. Unfortunately, these experiments were not carried out in the present study as they were considered too time consuming and costly to repeat.

\subsection{4 - MAO-A and MAO-B Gene Expression in U-118 MG Glioma Cells}

The present study used the U-118 MG human glioma cell line as an in vitro model of the physiological behavior of glial cells in the CNS, as discussed in section 2.1.2. The effects of tobacco smoke extracts on MAO-A and $-B$ gene expression were examined in this cell line, and were compared to the changes in gene expression induced by these extracts in the $\mathrm{SH}$ - 
SY5Y human neuroblastoma cell line. The hypothesis was that glia and astrocytes may respond differently to tobacco smoke than neuronal cells. Untreated U-118 MG cells expressed considerably lower levels of MAO-A and MAO-B mRNA than untreated SH-SY5Y cells. This is consistent with the earlier finding of this study that total MAO enzyme activity in this cell line was very low, and near the detection limits for the enzymatic assay used (section 5.3.4). As discussed previously, a number of studies have reported very high levels of MAO activity in astrocytes and glia in the CNS and therefore the poor enzyme activity measured in U-118 MG cells may indicate this cell line is an unsuitable model of in vivo glial functioning.

Although MAO-A and MAO-B mRNA levels were considerably lower in U-118 MG cells, these genes seemed to respond to the compounds tested in a similar manner to SH-SY5Y cells. No changes in MAO-A or MAO-B gene expression were observed in U-118 MG cells treated with ethanol for 5 days, and the relative expression levels of these genes were no different from the relative expression observed in SH-SY5Y cells treated with ethanol under the same regimen. Neither nicotine, nor QTPM caused any change in MAO-A expression in U-118 MG cells, and although HTPM induced a slightly significant up-regulation in U-118 MG cells, the relative expression in these cells was not significantly different from that seen in SH-SY5Y cells. Similarly, none of the tobacco compounds tested caused any change in MAO-B gene expression in U-118 MG cells or SH-SY5Y cells over 5 days.

These results indicate that nicotine and tobacco smoke extracts do not influence the regulation and expression of MAO-A and $-\mathrm{B}$ genes in glial cells. However, a major limitation of the current experiment is that gene expression was examined at only one time point. This decision was made because significant setbacks were encountered in culturing the U-118 MG cells, and resolving these difficulties proved very time consuming. The cells were treated using the continuous 5-day exposure period as it was initially thought that prolonged exposure to tobacco compounds would result in greater inhibition of enzyme activity, and therefore a more pronounced effect (if any) on gene expression. However, the gene expression and enzyme activity results obtained for SH-SY5Y cells demonstrated that treatment with the shorter 3-day exposure or the 5-day refreshed medium regimen were more effective at eliciting significant changes in MAO-A and -B mRNA expression. Therefore, it would be interesting to re-examine MAO gene expression in U-118 MG cells treated with under these exposure regimens to determine if these cells respond in the same manner as $\mathrm{SH}$ SY5Y. Nevertheless, any changes in U-118 MG gene expression in response to tobacco are academic if they do not provide insights into regulation of those genes in vivo. Before this cell 
line is used for further experiments with tobacco compounds, it should first be established whether U-118 MG is a suitable model for glial function and signaling in the CNS. This should involve experiments comparing the physiological function and gene expression of U-118 MG with primary cultures of glial cells.

\subsection{5-Summary}

The present study is the first to examine changes in MAO-A and MAO-B gene and protein expression in response to nicotine and tobacco smoke extracts. Exposure to tobacco smoke extracts caused a significant up-regulation in gene expression of both MAO-A and MAO-B enzymes in SH-SY5Y cells treated for 5 days although no corresponding increase in MAO-A or B protein levels was seen. These results suggest that chronic exposure to tobacco smoke may induce up-regulation of these genes in neuronal cells of human smokers, a possibility that has some support from the findings of Launay et al. (2009). Blockade of this increase by the $\mathrm{nAChR}$ antagonist, mecamylamine, indicated a potential role for $\mathrm{nAChR}$ in the up-regulation observed, even in response to nicotine-free smoke extracts. However, the findings of the present study should be interpreted with caution as the results obtained were highly dependent on the cell culture conditions used to maintain the cells. Nevertheless, the results obtained are intriguing, and warrant further investigation to determine the actual mechanism of MAO-A and MAO-B up-regulation in neuronal cells. 


\section{Chapter Seven: \\ Up-regulation of MOR gene expression following tobacco exposure}

The $\mu$ opioid receptor (MOR) is an integral membrane protein involved in many vital physiological and pharmacological functions (Loh \& Smith, 1990; Minami \& Satoh, 1995; Satoh \& Minami, 1995; Waldhoer et al., 2004). These include modulating respiratory rhythm, gastrointestinal mobility, pain transmission, and mediating positive reinforcement in learning behaviours. As discussed earlier (section 1.5.2), MOR activation can occur through the action of a number of endogenous neuronal peptides such as $\beta$-endorphin, enkephalin, and the endomorphins, and through the action of exogenous compounds and drugs of abuse. Activation may arise in the classical way through direct agonist binding to the receptor, as seen with morphine, or through indirect mechanisms as seen in MOR activation following cocaine, alcohol, or nicotine administration (Contet et al., 2004). To examine the relationship between MOR and nicotine and tobacco extract, a good understanding of normal MOR regulation is needed.

Changes in the expression of MOR mRNA have been reported following acute and chronic exposures to MOR agonists and antagonists. Much of the research in this area has focused on the effects of morphine, a plant-derived alkaloid drug used clinically as a potent analgesic. Morphine is the prototypical exogenous MOR agonist, selectively binding to MOR with high affinity, but also activating the other classical opioid receptors, delta and kappa. However, morphine also carries a high potential to induce physical dependence to the drug, and rapidly induces tolerance to its nociceptive effects. Previously it was thought that the biochemical mechanisms mediating morphine tolerance and dependence may be due to dynamic changes in receptor expression and desensitization. Many studies into MOR protein and gene expression have focused on changes in these parameters as a consequence of chronic agonist exposure. However, investigation of the effect of morphine on MOR mRNA expression has been far from straight-forward, and a number of inconsistent or contradictory findings have been reported. 
In vitro studies in cultured cells have found that mRNA expression of MOR is decreased after chronic exposure to morphine (X. Yu et al., 2003). Undifferentiated SH-SY5Y cells treated for 24 hours with $10 \mu \mathrm{M}$ morphine showed a $47 \%$ reduction in MOR mRNA expression compared to control cells, a decrease that was inhibited when morphine was co-administered with the opioid receptor antagonist naloxone (X. Yu et al., 2003). These results are in agreement with studies examining MOR binding density in SH-SY5Y cells following morphine treatment. Zadina et al. (1993; Zadina et al., 1994) measured the abundance of cell surface MOR protein in response to chronic morphine treatment by recording the specific binding of $\left[{ }^{3} \mathrm{H}\right] \mathrm{DAMGO}$ ([ $\left.{ }^{3} \mathrm{H}\right]$ Tyr-D-Ala-Gly-N-Me-Phe-Gly-ol), a radio-labelled MOR-specific agonist and enkephalin analogue. Morphine caused a significant dose-dependent decrease in $\left[{ }^{3} \mathrm{H}\right] \mathrm{DAMGO}$ binding in SH-SY5Y cells treated for 24 hours. MOR specific binding was reduced by up to $48 \%$ of control values in cells treated with the highest morphine concentration (Zadina et al., 1993; Zadina et al., 1994). This down-regulation of MOR was later found to be time-dependent, since morphine treatment for 2.5 hours reduced MOR-specific binding by $28 \%$, and by $50 \%$ after 24 hours treatment (Horner \& Zadina, 2004). Chronic morphine treatment also decreased $\left[{ }^{3} \mathrm{H}\right]$ DAMGO binding by $59 \%$ in $\mathrm{C}_{6}$ glial cells stably transfected to express MOR protein (Yabaluri \& Medzihradsky, 1997).

However, studies in vivo have reported variable findings for MOR abundance and expression following morphine treatment, showing either down-regulation or no change in MOR density. One study that examined rats chronically treated with i.p. morphine found no change in MOR mRNA expression in the brains of animals treated for 7 days (Brodsky et al., 1995). Similarly, in another study no change in DAMGO binding was observed in rats receiving morphine for 7 days (Patel et al., 2002). A third study found no significant differences in MOR mRNA expression in the striatum, nucleus accumbens, or septum of rats treated with increasing doses of morphine over 14 days (Castelli et al., 1997). In contrast, Teodorov et al. (2006), using RT-PCR technology to amplify MOR mRNA from various areas of the female rat brain, found regionally specific decreases in MOR gene expression following chronic morphine treatments. When rats were treated chronically with $3.5 \mathrm{mg} / \mathrm{kg}$ morphine (i.p.) for 5 days, reductions in MOR expression were seen in both the periaqueductal grey region and the striatum - areas of the brain implicated in morphine tolerance and dependence (Teodorov et al., 2006). Each of the above studies differed significantly with respect to morphine dosage, treatment duration, and experimental design, and these differences are likely to account for the differences in results obtained. However, taken together, these results suggest that any in 
vivo changes in MOR expression following morphine treatment may be transient and highly region-specific.

Interestingly, the effects of morphine on MOR mRNA expression differ markedly in comparison to the changes in MOR expression observed following chronic exposure to endogenous opioid peptides. Yu et al. (2003) demonstrated that chronic treatment with the endogenous MOR agonists endomorphin-1 and -2 significantly increased MOR mRNA expression. In SH-SY5Y cells treated with endomorphin-1, mRNA expression of MOR was increased by $370 \%$ compared to the control, and cells treated with endomorphin-2 showed increased expression of MOR to $250 \%$ of control. These increases were abolished when the test compounds were administered with naloxone. Interestingly, endomorphin-1 and -2 did not up-regulate MOR-specific binding density in SH-SY5Y cells when measured by $\left[{ }^{3} \mathrm{H}\right]$ DAMGO binding (Horner \& Zadina, 2004). Rather, the endomorphins reduced MOR-specific binding in a time- and dose-dependent manner, with $10 \mu \mathrm{M}$ endomorphin-1 inhibiting $\left[{ }^{3} \mathrm{H}\right.$ ]DAMGO binding to the cells by $72 \%$ compared to control. This observation was found to be due to receptor internalization, since down-regulation of MOR was reversed by treatment with hypertonic sucrose which inhibits internalization. Similarly, $\beta$-endorphin induced a similar decrease in MOR-specific binding following chronic treatment (Yabaluri \& Medzihradsky, 1997). Together, these results imply that chronic exposure to endogenous $\mu$ opioids causes internalization of MOR, and subsequent up-regulation of MOR gene expression occurs to restore basal levels of MOR expression in the plasma membrane.

The effects of endomorphin seem to be in contrast to the effects of morphine which either had no effect on transcription or caused a down-regulation of MOR abundance and mRNA expression. This may be explained by agonist-dependent receptor signaling, a mechanism that was first described for MOR (Contet et al., 2004; McConalogue et al., 1999). When an agonist binds to MOR, the agonist-receptor complex determines the physiological outcome of ligand binding, rather than the receptor itself. Through this mechanism, several different biological outcomes are possible following activation of one receptor type. It is thought that morphine binding stabilizes MOR in a different conformation to that induced by endogenous MOR agonists, leading to initiation of different signal transduction pathways and different intracellular sequelae (McConalogue et al., 1999). This is particularly evident with respect to receptor internalization, as the endogenous $\beta$-endorphin, endomorphins, and enkaphalins cause a rapid internalization of MOR, but morphine does not (Keith et al., 1997; Keith et al., 1996; McConalogue et al., 1999). Horner \& Zadina (2004) demonstrated that morphine is 
capable of inducing receptor internalization in SH-SY5Y cells, but this response was considerably slower than that seen with endomorphin-1.

\subsection{2-Drugs of Abuse}

Changes in the expression of MOR have also been studied with respect to a number of other non-opiate drugs of abuse. The effects of cocaine in particular have been studied extensively, and this research is briefly reviewed here to provide insight into the effects of non-opiate drugs on the expression of MOR.

Similar to the changes induced by endogenous opioid peptides, chronic cocaine exposure also induces a significant up-regulation of MOR receptor binding and MOR gene expression (Azaryan et al., 1996a; Azaryan et al., 1996b; Schroeder et al., 2003). However, unlike morphine or the endogenous opioid peptides, cocaine does not bind to MOR but exerts its neurochemical effects through binding to the dopamine transporter (DAT) and inhibiting the re-uptake of dopamine from the synaptic cleft. This results in increased dopamine accumulation in the synapse, particularly in the nucleus accumbens, which induces the rewarding properties of the drug (Di Chiara et al., 2004).

Azaryan et al. (1996a, 1996b) demonstrated that continuous cocaine administration for 3 days increased MOR mRNA expression in the nucleus accumbens of treated rats by at least $350 \%$ of control. Co-administration of cocaine with D1 and D2 selective dopamine receptor antagonists abolished the observed increase in MOR mRNA expression, demonstrating that up-regulation of MOR gene expression following chronic cocaine administration was dependent on dopaminergic mechanisms. Thus, it was postulated that increases in MOR gene expression may be a result of excess synaptic dopamine concentrations induced by cocaine (Azaryan et al., 1996a). This would also suggest that other drugs of abuse that cause dopamine overflow in the accumbens may induce changes in MOR gene expression. In fact this appears to be the case. Although there has been little research in this area, studies have reported that ethanol exposure can decrease MOR mRNA expression in some areas of rat brain (Winkler et al., 1998), amphetamine causes up- and down-regulation of MOR mRNA in different regions of rat brain (Vecchiola et al., 1999), and cannabinoids up-regulate MOR mRNA expression in mouse brain (Paldyova et al., 2007). One theory is that these changes are 
due to a drug-induced increase in the endogenous MOR ligands, $\beta$-endorphin and the endomorphins, following increased dopamine release (Roth-Deri et al., 2008). Thus, the observed MOR up-regulation may result from endogenous opioid ligand activation of the receptor.

If this is the case, one would expect chronic nicotine and tobacco exposure to also induce upregulation of MOR. Since like cocaine, nicotine induces dopamine overflow in the nucleus accumbens, it may also cause up-regulation of endogenous opioid peptides, leading to increased MOR gene expression. Several studies have reported an increase in opioid peptide levels in the brains of rodents exposed to nicotine (Dhatt et al., 1995; Houdi et al., 1998). Dhatt et al. (1995) treated mice acutely with a single dose of $1 \mathrm{mg} / \mathrm{kg}$ nicotine before sacrificing the animals up to 24 hours later. Mice were also treated chronically with the same dose of nicotine daily for 14 days before sacrifice. In contrast, Houdi et al. (1998) treated Sprague-Dawley rats with $0.6 \mathrm{mg} / \mathrm{kg}$ nictoine once before sacrificing the rats up to six hours later, and chronically treated rats were treated twice daily for 14 days. These studies examined changes in the expression of preproenkephalin (PPE) mRNA, a precursor of the endogenous MOR ligand enkephalin, in response to different nicotine treatments. Both chronic and acute nicotine treatments increased the expression of PPE in the striatum and hippocampus of rodents. However, in contrast, another study by Wewers et al. (1999) treated rats with $0.3 \mathrm{mg} / \mathrm{kg}$ of nicotine three times daily for 14 days, and found that chronic nicotine treatment decreased met-enkephalin levels in the striatum of rats by up to $50 \%$ (Wewers et al., 1999).

The results of research examining the effects of nicotine treatment on $\beta$-endorphin release are similarly inconsistent. Early studies reported that cigarette smoking caused an increase in the plasma levels of $\beta$-endorphin in smokers (Pomerleau et al., 1983a), and that nicotine also stimulated the release of $\beta$-endorphin in isolated, perfused mouse brain (Marty et al., 1985). However, another study found that nicotine increased $\beta$-endorphin secretion from cultured hypothalamic neurons following treatments of up to 24 hours, but longer treatments caused desensitization of the $\beta$-endorphin response (Boyadjieva \& Sarkar, 1997). Additionally, Gilbert et al. (1992) found that $\beta$-endorphin plasma concentrations in human smokers were not elevated when smoking their own brand of cigarette, but were increased when smoking cigarettes with a higher nicotine concentration. However, Wewers et al. (1994) found no correlation between changes in plasma $\beta$-endorphin levels and periods of smoking or abstinence in dependent smokers. These results suggest that $\beta$-endorphin exhibits a bi-phasic 
response to nicotine (Berrendero et al., 2010); however, to confirm this theory more research into the effects of nicotine on $\beta$-endorphin release is warranted.

Very little research has yet been conducted on the effects of tobacco or nicotine on the expression of opioid peptides or MOR. This is despite a number of studies providing convincing evidence for the involvement of MOR in the mediation of tobacco dependence (detailed in section 1.5.3). Wewers et al. (1999) measured MOR specific binding in the striatum of rats treated chronically with nicotine for 14 days and found a significant $85 \%$ increase in MOR binding in rats receiving nicotine treatment, compared to control. It was thought that this was a compensatory response to the observed decreases in striatal metenkephalin concentrations following chronic nicotine exposure.

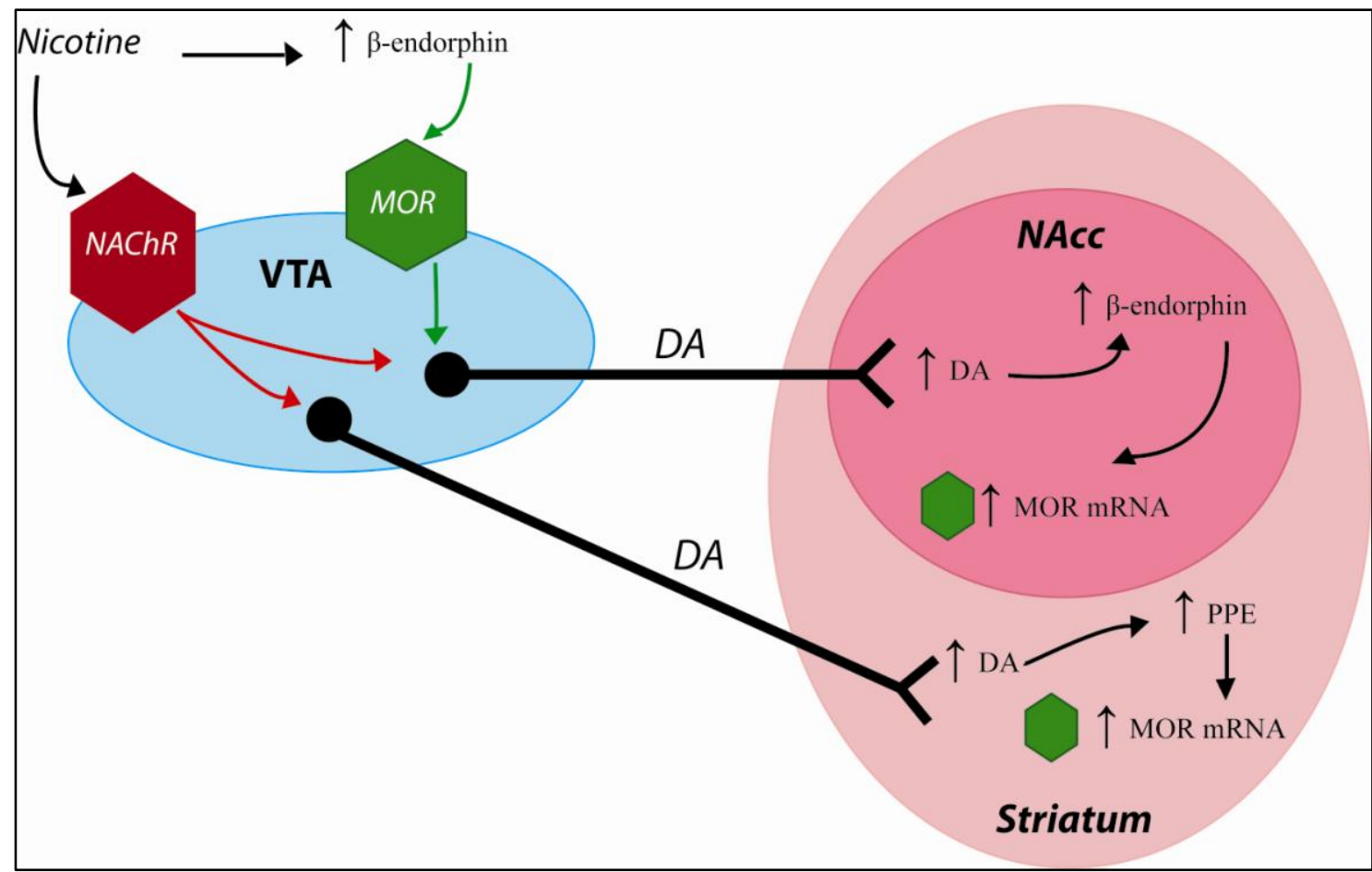

Figure 56: Schematic diagram of the proposed interaction between dopamine (DA) and the endogenous opioid system in the mesolimbic dopamine system. Nicotine binding in the Ventral Tegmental Area (VTA) causes dopamine overflow in the Nucleus accumbens (NACC) and other striatal regions. Excess dopamine leads to release of the endogenous opioid peptides $\beta$-endorphin and preproenkephalin (PPE) which causes internalization and up-regulation of MOR. Increased $\beta$-endorphin release in response to nicotine ia also thought to activate MOR in the VTA, modulating the firing of DA projections to the Nacc.

To date only one study has examined the effects of nicotine on MOR mRNA expression. Walters et al. (2005) measured MOR mRNA in the ventral tegmentum (VTA) and nucleus accumbens of mice treated with saline, nicotine, or cocaine for 8 days. Mice chronically treated with nicotine showed significant up-regulation of MOR mRNA in the VTA, to 
approximately $150 \%$ of the expression seen in saline-treated rats. This up-regulation was found to be dependent on MOR activation, as naloxone pre-treatment blocked the response. Furthermore, increased MOR mRNA expression was also found to be a nicotine-specific response, as cocaine treatment did not induce MOR up-regulation in this study (Walters et al., 2005). Additionally, this study showed that activation of the transcription factor CREB was required for MOR mRNA up-regulation, since mice carrying a mutant form of CREB showed no increase in MOR mRNA expression in response to nicotine. CREB is thought to play a major role in the rewarding properties of many drugs of abuse, and the results from this study suggest that a CREB-dependent increase in MOR expression is required for nicotine reward (Walters et al., 2005).

The mechanisms and pathways governing the interaction between nicotine and the opioidergic system are complex and not yet well characterized. There is convincing evidence that MOR activation is necessary for the reinforcing properties of nicotine; however, nicotine is not known to directly interact with MOR. It has been suggested that the increased dopamine concentrations induced by nicotine binding to the acetylcholine receptor (nAChR) may lead to increased release of endogenous opioid peptides such as $\beta$-endorphin or the endomorphins (Fig. 56). In turn, these opioid peptides mediate the activation of MOR in regions of the brain critical to the establishment of drug dependence and reinforcement. However, further research is still required to properly elucidate the mechanisms behind the involvement of MOR in mediating nicotine reward.

\section{$\underline{7.1 .3-\text { Objectives }}$}

The aim of this study was to examine the effects of nicotine, HTPM and QTPM on the gene expression of MOR in cultured SH-SY5Y cells. While a prior study has reported in vivo changes in MOR gene expression in rodents treated with nicotine (Walters et al., 2005), the present study aimed to examine whether these reported changes were also observed in a cultured human neuroblastoma cell line, and to determine whether whole tobacco extracts induced the same changes in gene expression as seen with nicotine.

As there is strong evidence that MOR is linked to the activation of nAChRs, this study planned to investigate whether $n A C h R$ activation was required for nicotine's effects on MOR gene expression. To determine if changes in MOR expression were a downstream result of nicotine binding to nAChRs, or if they were mediated through a nAChR-independent signaling pathway, 
an adaptation of a previous experiment by Dunckley \& Lukas (2006) using the nAChR antagonist mecamylamine was planned. If changes in MOR expression involve nAChRdependant signaling pathways, then treatment with mecamylamine should reverse any changes in MOR expression observed. Additionally, if changes in MOR expression are due to a nicotine-dependent mechanism, then it is expected that QTPM extract should have no effect on MOR expression, as QTPM lacks nicotine.

Quantitative real-time PCR was used to analyze the expression of MOR in SH-SY5Y cells chronically treated with nicotine, HTPM and QTPM. These data were used to assess the effect of tobacco smoke exposure on the expression of MOR. Additionally, SH-SY5Y cells were treated with tobacco compounds in the presence of mecamylamine to ascertain whether changes in MOR gene expression associated with tobacco extract exposure occur in a nAChRindependent manner. It was hoped that these experiments would add to our understanding of the nicotine and non-nicotine related effects of tobacco smoke, and perhaps strengthen the evidence for MOR involvement in tobacco dependence.

\section{2-MATERIALS \& METHODS}

\subsection{1 - Tobacco Particulate Exposures}

Cultured SH-SY5Y cells were exposed to ethanol, nicotine, HTPM or QTPM for 1, 3, or 5 days according to the protocol detailed in section 2.2.2. Briefly, $9.8 \mu \mathrm{L}$ volumes of nicotine or HTPM extract were added to growing SH-SY5Y cells in $30 \mathrm{~mL}$ of growth medium (RPMI 1640, 10\% FCS, 1\% PenStrep; see section 2.1.3), to give a final concentration of $0.2 \mu \mathrm{M}$ nicotine. Equivalent volumes of QTPM or ethanol were added to other flasks, bringing the final ethanol concentration in each flask to $5.6 \mathrm{mM}$. Flasks of cells were incubated for 1,3 , or 5 days continuously, without disruption, before harvesting for total RNA extraction, according to the methods described in section 2.3.

These exposure groups included a flask each of untreated SH-SY5Y, ethanol-treated, nicotinetreated, HTPM-treated, and QTPM-treated cells incubated for a given time period. Seven exposure groups each were prepared for the 1-day time point and the 3-day time point, and thirteen treatment groups were prepared for the 5-day time point. 


\subsubsection{2 - 5-day Refreshed Medium Exposure Groups}

Six flasks of each treatment were also prepared using the refreshed-medium treatment regimen (detailed in section 6.2.1.3), in which the medium was changed on day 3 of the 5 -day exposure period. On the third day, cells received a fresh aliquot of culture medium and tobacco test compound, and were then returned to the incubator for a further 2 days incubation. Six independent flasks of each treatment were prepared.

\subsubsection{3 - Mecamylamine Exposure Groups}

Flasks of cells were exposed to ethanol and tobacco compounds in the presence of $10 \mu \mathrm{M}$ mecamylamine. Six flasks each were treated with mecamylamine, mecamylamine and ethanol $(5.6 \mathrm{mM})$, mecamylamine and nicotine $(0.2 \mu \mathrm{M})$, mecamylamine and HTPM, or mecamylamine and QTPM. The HTPM dosage was sufficient to give a $0.2 \mu \mathrm{M}$ nicotine concentration, and an equivalent volume of QTPM was used. Flasks were incubated for 3 days continuously before harvesting for RNA extraction, according to the method described in section 2.3.

\subsection{2 - Quantitative Real-Time RT-PCR}

Upon completion of the cell culture treatment regimens, cells were lysed in TRIzol ${ }^{\circledR}$ reagent, and the total RNA was extracted and transcribed into CDNA according to the methods described in section 2.6. The cDNA was assayed for MOR gene expression using customized hMOR-specific primers that were designed and fully validated for this assay. This involved assessment of the amplification efficiency and product specificity, as described in section 6.1.3.

Gene expression data for MOR in treated cells were expressed as fold changes relative to a calibrator sample (RQ). For ethanol-treated cells the calibrator sample used was untreated SH-SY5Y cells, and for cells treated with nicotine, HTPM, or QTPM, cDNA from ethanol-treated cells was used as the calibrator to eliminate any effects of the ethanol vehicle. POLR2F had been previously validated as a stable reference gene (section 6.3.1.2) and was also used as the reference gene for all experiments in this study. 


\subsection{3 - Western Blotting}

Western blotting was performed on cell protein lysates to determine if changes in MOR gene expression correlated with MOR protein abundance. Protein lysates prepared from cells receiving the 5-day refreshed treatment regimen were used, as these cells showed the greatest change in MOR gene expression. If changes in gene expression were correlated with protein abundance the 5-day refreshed treatment groups should show the greatest change in MOR protein levels.

Four samples each of ethanol, nicotine, HTPM, and QTPM-treated cell extracts from four different culture experiments were assayed for MOR protein by Western blot. One of each treatment was tested per blot, for a total of four independent Western blotting experiments. Western blots were immunostained using a rabbit polyclonal antibody (Chemicon Intl.; AB5511) specific for a C-terminus epitope in the rat MOR protein. A mouse polyclonal anti- $\beta$-tubulin antibody (BD Pharmingen; cat. 556321) was used as a protein loading control. The MOR antibody produces specific bands at approximately $50 \mathrm{kDa}$ and $70 \mathrm{kDa}$ in SH-SY5Y cells (Kivell et al., 2004), and the $\beta$-tubulin antibody produces a band at $50 \mathrm{kDa}$. Because both antibodies yield a band near $50 \mathrm{kDa}$, hMOR was visualized with a $\mathrm{Cy}^{\circledR}-5$ labeled anti-rabbit secondary antibody, and $\beta$-tubulin was visualized with a $\mathrm{Cy}^{\circledR}-3$ labeled anti-mouse antibody. The fluorescence from these bands was scanned using a Fujifilm FLA-5100 imaging system (Fuji Photo Film Co., Ltd). Cy ${ }^{\circledR}-5$ fluorescence was measured with a 653 nm laser and the DBR1/R665 emission filter, and $\mathrm{Cy}^{\circledR}-3$ fluorescence was scanned with a $532 \mathrm{~nm}$ laser and PBG/570DF20 emission filter. Images of the blots were analyzed by integrated pixel density densitometry on 16-bit TIFF images using ImageJ v3.5 software (Wayne Rasbond, National Institutes of Health, USA). Specific Western blotting procedures are detailed in section 2.5 of this thesis.

\section{$\underline{7.2 .4-D a t a ~ A n a l y s i s}$}

The $\mathrm{Ct}$ values obtained from qRT-PCR experiments were used to calculate fold changes in hMOR gene expression, expressed as relative quantitation (RQ). The efficiency adjusted Ct method was used as described in section 6.1.3.2. 


\section{$\underline{7.3-\text { RESULTS }}$}

\subsection{1 - Validation of the qRT-PCR Assay}

Primers to specifically amplify a $230 \mathrm{bp}$ segment of the human OPRM1 gene (MOR) were designed as described in section 2.6.4. To demonstrate the specificity of these primers a PCR product melt-curve analysis was performed with each RT-PCR assay, and the PCR products were analyzed by agarose gel electrophoresis. The hMOR PCR melt-curve consistently and reliably gave a single peak at $83.6^{\circ} \mathrm{C}$ (Fig 57a). Negative PCR controls containing water in place of cDNA template showed no amplification, and no fluorescence peak was observed in meltcurve analysis, indicating that the primers only amplified the intended target.

(a)

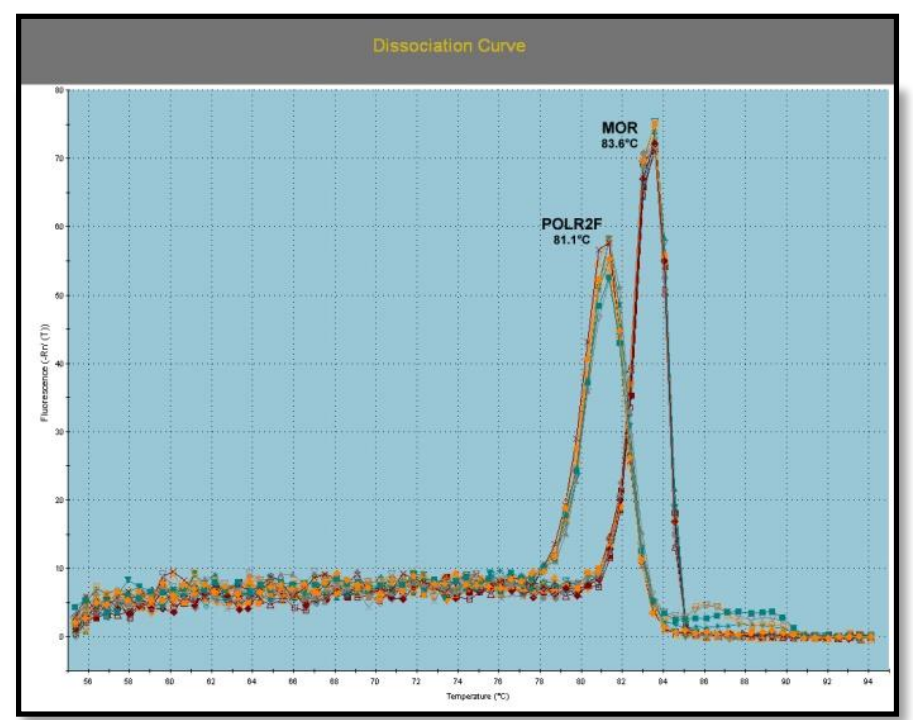

(b)

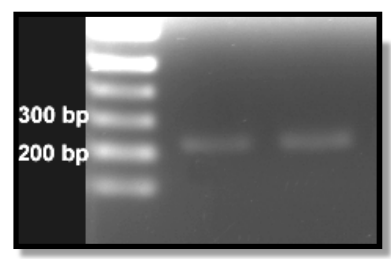

Figure 57: (a) Representative dissociation plot demonstrating the melt-curves for MOR and POLR2F genes amplified from SH-SY5Y cells. (b) Electrophoretic bands of MOR PCR products following agarose gel electrophoresis and ethidium bromide staining. The MOR-specific amplicon bands measure $230 \mathrm{bp}$ in size. Lane 1 = MW Ladder, Lanes 2 and 3 = MOR PCR product.

Electrophoretic separation of the MOR PCR products on $1 \%$ agarose gels yielded only a single band of DNA at the expected PCR product size of approximately $230 \mathrm{bp}$ (Fig. 56b). Together these results demonstrate that the designed PCR primers were specifically amplifying the target segment of OPRM1 from SH-SY5Y-derived mRNA. The amplification efficiency of the PCR reaction using the MOR primers was determined by serially diluting an SH-SY5Y cDNA sample before amplification. The amplification efficiency was approximately 98.4\% (Appendix 
9.4). Amplification efficiency and specificity was previously confirmed for the POLR2F primers, and is presented in section 6.3.1.

\subsection{2 - Effects of Ethanol Exposure on MOR Gene Expression}

The relative gene expression of MOR was assayed in SH-SY5Y cells that had been exposed to absolute ethanol (5.6 mM) for 1, 3, or 5 days continuous exposure. After 1 day MOR mRNA expression increased modestly by $22 \%$, but this was not statistically significant (Fig. 58 , Table 19). After 3 days of continuous treatment, MOR expression fell significantly to $53 \%$ of control MOR expression (one-way ANOVA with Bonferroni's post-test; $p<0.05$ ). Following 5 days of continuous ethanol exposure MOR gene expression increased slightly from the 3 day expression levels to $84 \%$ of untreated controls. This change was not significantly different from the control or from the 3-day treatment group.

Table 19: Summary of MOR relative expression in ethanol-treated SH-SY5Y cells

\begin{tabular}{lccc}
\hline Treatment & $\mathrm{RQ}$ & $\mathrm{SEM}$ & $N$ \\
\hline 1 day & 1.22 & 0.10 & 7 \\
3 days & $0.53^{*}$ & 0.04 & 7 \\
5 days & 0.84 & 0.09 & 13 \\
5 days refreshed & 1.16 & 0.16 & 5 \\
\hline
\end{tabular}

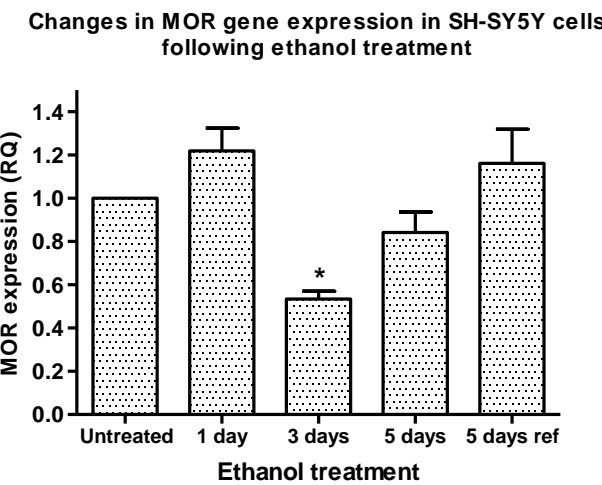

Table 19: Summary of relative expression data, where sample size is expressed as $n$ independent preparations. Data were analyzed by one-way ANOVA with Bonferroni's multiple comparison post-test $(\alpha=0.05)$.

Figure 58: Changes in MOR gene expression in cultured SH-SY5Y cells following exposure to ethanol (5.6 $\mathrm{mM}$ ) expressed as quantitation relative to the POLR2F reference gene (RQ). MOR gene expression in cells treated with ethanol for 3 days was significantly down-regulated ( $* P<0.05)$ to $53 \%$ of the expression in untreated cells. MOR transcript levels were not significantly different from controls in cells treated with ethanol for 1 or 5 days continuously, or over the 5-day refreshed treatment regimen.

As described above, MOR gene expression decreased significantly after 3 days of ethanol treatment before returning to near control levels after 5 days of continuous treatment. This rebound effect was unexpected and may be due to natural variation in the samples. It was 
thought that the ethanol may be subject to metabolism and clearance by the cells before the completion of the 5-day incubation period. This might explain why MOR mRNA expression had returned to near-control levels at 5 days. MOR gene expression in cells treated with ethanol (5.6 $\mathrm{mM}$ ) over the 5-day refreshed medium regimen was not significantly different from untreated controls, nor to cells exposed for 5 days continuously. However, expression was significantly different from cells treated with ethanol for 3 days (one-way ANOVA with Bonferroni's multiple comparison post-test; $\mathrm{P}<0.01$ ).

\subsection{3 - Effects of Tobacco Extract Exposure on MOR Gene Expression}

Nicotine had no measurable effect on the gene expression of MOR over 1, 3, or 5 days of continuous exposure (Fig 59). MOR mRNA expression remained at control levels over all time points, and the variance in the data was appropriately small. Exposure to HTPM and QTPM caused a slight decrease in mRNA levels after 1 day, but this was not statistically significant. After 3 days treatment with HTPM and QTPM, MOR expression increased by $27 \%$ and $30 \%$, respectively. These increases were modest, but statistically significant in both HTPM- and QTPM-treated groups. After 5 days of continuous treatment, gene expression in HTPM- and QTPM-treated cells returned to near-control levels of expression (two-way ANOVA with Bonferroni's post-test; $\alpha=0.05$ ).

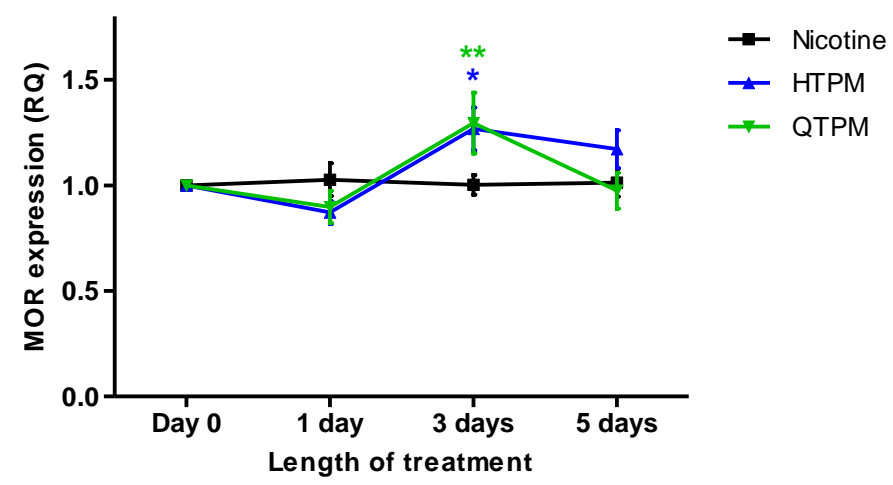

Figure 59: Changes in MOR gene expression in cells treated with various tobacco constituents for up to 5 days. Exposure to $0.2 \mu \mathrm{M}$ nicotine elicited no change in MOR gene expression in cells treated for 1,3 , or 5 days continuously. Cells treated with HTPM or QTPM showed up-regulation of MOR mRNA expression after 3 days treatment, but expression fell back to control levels after 5 days treatment. Data were tested by two-way ANOVA with Bonferroni's multiple comparison post-test; $n \geq 6$ independent preparations for all conditions. ${ }^{*} \mathrm{P}<0.05 ;{ }^{* *} \mathrm{P}<0.01$. 
MOR mRNA expression was also examined in cells treated with tobacco constituents over the 5 day-refreshed medium time course. These results were compared to the 1-, 3-, and 5-day continuous treatment groups (Fig. 60a-d). MOR gene expression in cells treated with the refreshed medium regimen increased significantly in response to all treatments.

Nicotine-treated cells showed a significant $51 \%$ increase in MOR gene expression, compared to controls. This level of MOR expression was significantly different from controls, and from the expression of cells treated with nicotine for 5 days continuously $(P<0.001)$. MOR mRNA levels increased by 65\% in HTPM-treated cells, and in QTPM-treated cells expression was increased by $116 \%$ above control. Both of these changes were significant increases over control expression levels, and were significantly different compared to the MOR gene expression measured in cells treated for 5 days continuously ( $P<0.001)$ (Fig. 61).

(a)

Effect of nicotine exposure on MOR gene expression in cultured SH-SY5Ycells.

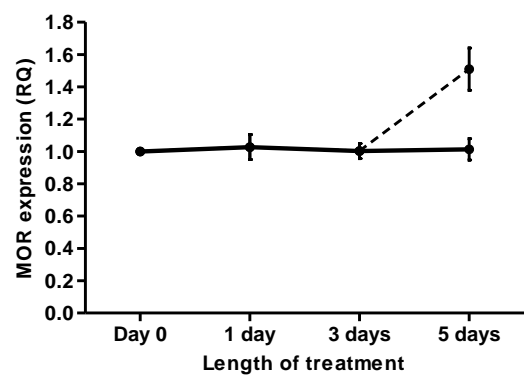

(c)

Effect of QTPM exposure on MOR gene expression

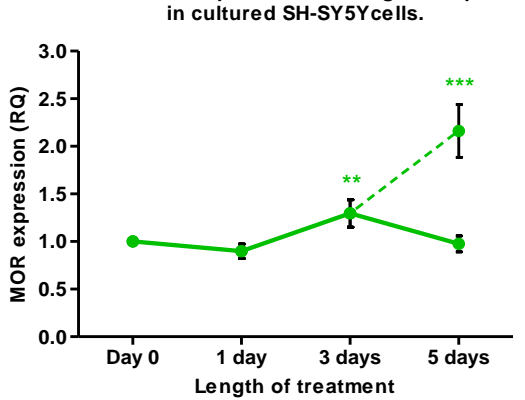

(b)

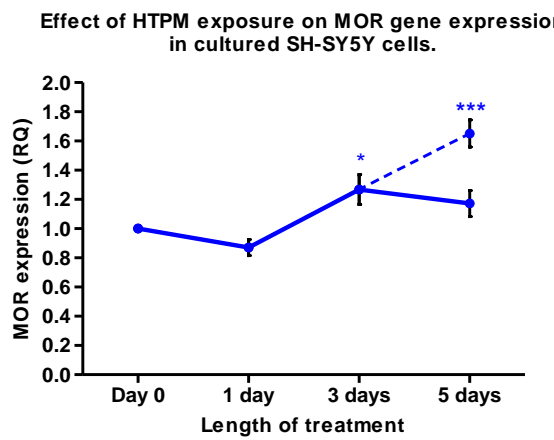

(d)

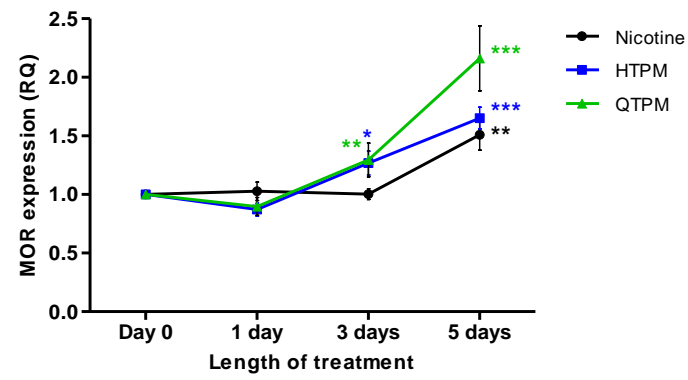

Figure 60: Changes in MOR gene expression in SH-SY5Y cells following treatment with (a) $0.2 \mu \mathrm{M}$ nicotine, (b) HTPM, and (c) QTPM. Solid lines connect data points from Fig. 58 presented earlier for cells treated for 1,3 , or 5 days continuously, and dashed lines represent gene expression values for cells treated with the refreshed medium regimen. Changes in expression are compared for 1-day, 3-day, and refreshed treatments in (d). ${ }^{*} \mathrm{P}<0.05 ;{ }^{* *} \mathrm{P}<0.01 ;{ }^{* * *} \mathrm{P}<0.001$; two-way ANOVA with Bonferroni's multiple comparison post-test; $\mathrm{n} \geq 5$ preparations for all conditions. 


\subsubsection{1 - Summary of Changes in MOR Expression}

The changes in MOR mRNA expression following these exposures suggest that both nicotine and the non-nicotine components of tobacco smoke contribute to up-regulation of MOR following exposure to tobacco. Overall gene expression data for MOR following treatment are summarized in Table 20.

Table 20: Summary of MOR gene changes following TPM exposure

\begin{tabular}{l|ccccccccccc}
\hline \multirow{2}{*}{ Treatment } & \multicolumn{3}{c}{ Nicotine } & \multicolumn{3}{c}{ HTPM } & \multicolumn{3}{c}{ QTPM } \\
& RQ & SEM & $\mathrm{N}$ & RQ & SEM & $\mathrm{n}$ & RQ & SEM & $\mathrm{n}$ \\
\hline 1 day & 1.03 & 0.07 & 7 & 0.87 & 0.05 & 6 & 0.90 & 0.07 & 7 \\
3 days & 1.00 & 0.05 & 6 & $1.27 *$ & 0.10 & 6 & $1.30 *$ & 0.14 & 7 \\
5 days & 1.01 & 0.07 & 11 & 1.17 & 0.08 & 11 & 0.98 & 0.08 & 10 \\
5 day refreshed & $1.51 *$ & 0.13 & 5 & $1.65 *$ & 0.09 & 6 & $2.16 *$ & 0.27 & 6 \\
\hline
\end{tabular}

Relative fold changes in MOR gene expression in SH-SY5Y cells treated with tobacco constituents. Sample sizes for each condition are expressed as independently prepared flasks ( $n$ ). ${ }^{*} P<0.05$;

MOR mRNA expression in nicotine-treated and HTPM-treated cells was significantly increased compared to ethanol-treated controls; however, there was no significant difference in MOR expression between nicotine-treated and HTPM-treated cells. MOR expression in cells exposed to QTPM for 5 days with refreshed media increased to $216 \%$ of control levels, which was statistically significant compared to control expression and expression in nicotine-treated cells $(P<0.01)$ (Fig. 61). This indicates that there is a significant difference between the effect of nicotine, and the effect of non-nicotine components of tobacco on the gene expression of MOR in SH-SY5Y cells. 


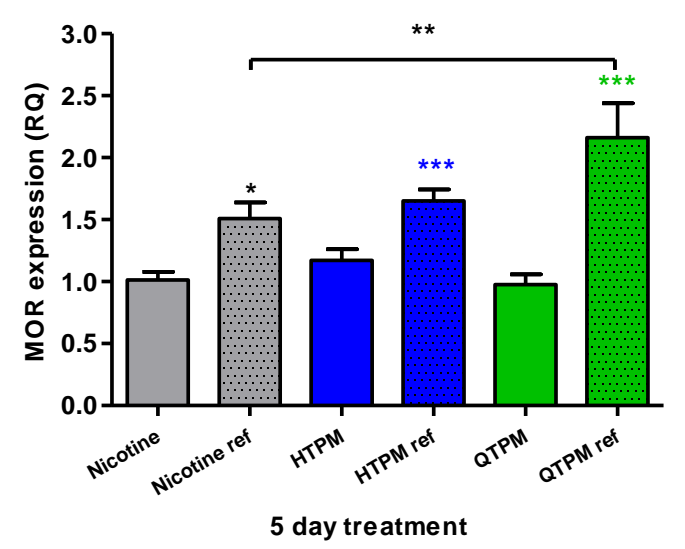

Figure 61: Comparison of MOR gene expression in SH-SY5Y cells treated with nicotine, HTPM, or QTPM for 5 days continuously, or with refreshed medium (ref). This figure compares data from figures 59a-c. Significant increases in expression were observed in all treatments using the refreshed medium regimen (one-way ANOVA with Bonferroni's multiple comparison post-test. ${ }^{*} \mathrm{P}<0.05$; ${ }^{* *} \mathrm{P}<0.01$; ${ }^{* * *} \mathrm{P}<0.001 . \mathrm{n} \geq$ 5 preparations for all conditions). Expression in cells treated with QTPM over the refreshed regimen was also statistically different from MOR expression in cells receiving nicotine under the same regimen, but not significantly different from HTPM.

\subsection{4 - Effects of Mecamylamine}

Treatment with $10 \mu \mathrm{M}$ mecamylamine alone induced a significant increase in MOR gene expression in SH-SY5Y cells (Fig. 61a). In cells treated for 3 days with mecamylamine alone, expression of MOR was increased by $93 \%$ above controls, and in cells treated with mecamylamine and ethanol together MOR mRNA expression was $85 \%$ greater than untreated control cells. Therefore, SH-SY5Y treatment groups receiving tobacco compounds in the presence of mecamylamine were normalized with the cells treated with mecamylamine and ethanol together. The aim of this was to correct for the direct effects of mecamylamine and ethanol on the cells, and allowed analysis of the changes in gene expression that were specifically due to exposure to the tobacco compounds.

SH-SY5Y cells treated with nicotine in conjunction with mecamylamine showed no significant change in MOR gene expression after 3 days exposure (Fig. 61b), consistent with the response seen in cells treated with nicotine alone. Cells treated with HTPM for 3 days showed MOR expression increased to $127 \%$ of control (section 7.3.3), but when cells were exposed to HTPM in the presence of mecamylamine this increase in expression was abolished. The relative expression of MOR in Mec-HTPM treated cells was measured to be 1.0, or $100 \%$ of control. However, the difference in expression between HTPM-treated and HTPM and mecamylamine- 
treated cells was not found to be statistically significant, due to variation in the data. Cells treated with QTPM for 3 days showed a significant increase in MOR expression to $130 \%$ (section 7.3.3), but in cells treated with mecamylamine and QTPM together, MOR expression fell quite significantly to $48 \%$ of untreated controls.

(a)

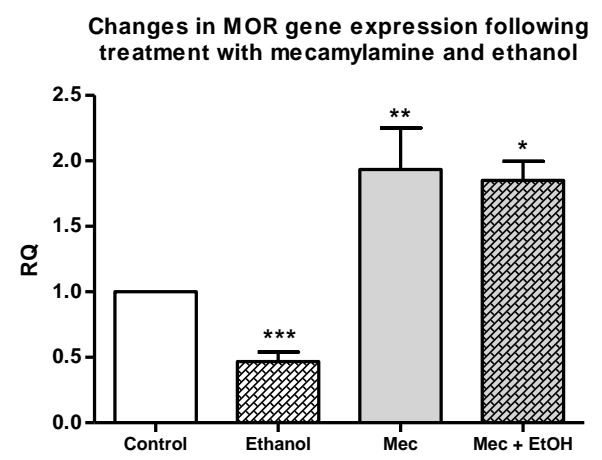

(b)

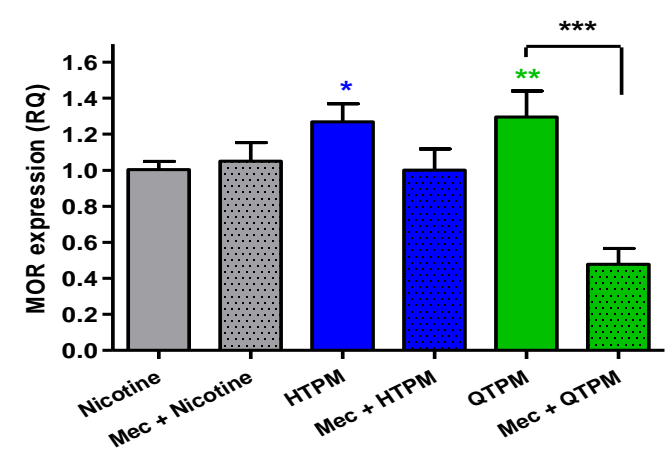

Figure 62: (a) MOR gene expression was significantly increased in SH-SY5Y cells treated with mecamylamine, or mecamylamine and ethanol together, for 3 days. (b) Increases in MOR gene expression in cells treated with HTPM and QTPM for 3 days (presented in section 7.3.3) were abolished in cells treated with these extracts in conjunction with mecamylamine. (One-way ANOVA with Bonferroni's multiple comparison post test; $\mathrm{n} \geq 6$ independent preparations for all conditions. ${ }^{*} \mathrm{P}<0.05 ;{ }^{*} \mathrm{P}<0.01$; $* * * \mathrm{P}<0.001$.)

The results of this experiment demonstrate that the observed increases in MOR gene expression following treatment with HTPM and QTPM tobacco extracts appear to be blocked by the action of mecamylamine. This indicates that tobacco extracts induce an up-regulation of MOR, that is dependent on the activation of nAChRs.

\subsection{5 - Western Blotting}

Western blotting was performed with a MOR-specific antibody to determine whether the observed increases in MOR mRNA expression following HTPM and QTPM exposure corresponded to increased concentrations of MOR protein. Appropriate secondaryantibody-only controls were performed, and showed no non-specific staining, verifying the specificity of the MOR primary antibody. SH-SY5Y protein lysates treated with nicotine, HTPM, and QTPM for 5 days with the media refreshed at day 3 showed a consistent increase in MOR-specific band density relative to the ethanol-treated control (Fig. 63c). 
(a)

(b)

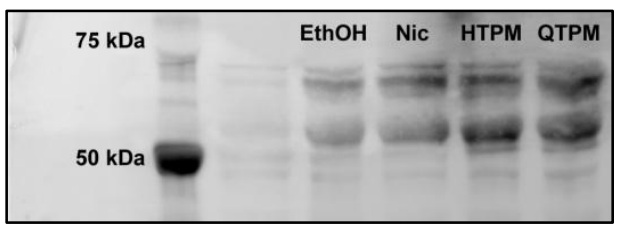

$75 \mathrm{kDa}$

$50 \mathrm{kDa}$ (c)

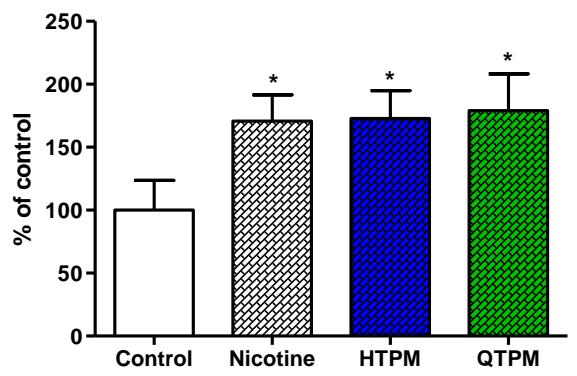

Figure 63: Western blots of MOR protein in SH-SY5Y cells treated for 5 days with refreshed medium. (a) A representative blot showing MOR-specific bands at approximately $55 \mathrm{kDa}$ and $70 \mathrm{kDa}$ in lysates from cells treated with the ethanol control, nicotine, HTPM, and QTPM. This blot was scanned with a $653 \mathrm{~nm}$ red laser to visualize the Cy-5 labeled secondary antibody. (b) The same blot scanned with a $532 \mathrm{~nm}$ green laser to visualize $\mathrm{Cy}-3$ stained bands for the $\beta$-tubulin protein loading control. (c) Relative electrophoretic band densities for MOR-stained Western blots expressed as percent of the ethanol control (mean + SEM). Cells treated with nicotine, HTPM, and QTPM showed a greater abundance of MOR compared to ethanol-treated cells (one-tailed Student's t-tests; $n=4$ blots prepared independently; * $\mathrm{P}<0.05)$.

Relative densities of the MOR-specific bands were measured with ImageJ software, and normalized using the density of the $\beta$-tubulin specific bands. Nicotine, HTPM and QTPM treated cells consistently yielded MOR-specific bands that were of a higher relative density than ethanol treated cells. The relative MOR band density for nicotine, HTPM and QTPM treated cells were $171 \%, 173 \%$, and $179 \%$, respectively. These differences were found to be statistically significant increases when compared to the ethanol control. A large amount of variation was observed in the data, known to be a common limitation of semi-quantitative Western blotting.

\section{4- DISCUSSION}

\subsection{1 - Effects of Ethanol on MOR Gene Expression}

Significant changes in MOR gene expression were observed in SH-SY5Y cells exposed to ethanol continuously for 3 days. MOR expression was down-regulated by approximately $53 \%$ 
in these cells relative to the untreated SH-SY5Y controls. These findings are consistent with previous studies reporting down-regulation of MOR in the brains of animals chronically treated with ethanol. Winkler et al. (1998) reported MOR mRNA expression was downregulated by almost $80 \%$ in the striatum of mice allowed to drink ethanol ad libitum for four weeks, and Saland et al. (2005) described significantly lower levels of immunohistochemical staining for MOR in multiple brain regions, including the striatum and nucleus accumbens, in rats administered ethanol for two weeks. Although these in vivo studies may reflect the indirect effects of ethanol on MOR, they may still relate to the down-regulation observed in the present study.

Interestingly, cells treated with ethanol for 1 or 5 days in the present study did not display down-regulation of MOR gene expression. It is possible that the shorter duration exposure of 24 hours is insufficient for the cells to respond and alter MOR gene transcription at this ethanol concentration. The absence of down-regulation at the 5 day time is difficult to explain, since the studies described above reported down-regulation of MOR following chronic ethanol exposure. However, those studies examined changes in the brains of rodents, while SH-SY5Y is an immortalized human cell line, and interspecies differences in MOR expression are known to exist (Lee et al., 2004; Peckys \& Landwehrmeyer, 1998). It is likely that after 5 days incubation ethanol concentrations in the cultures had fallen to sub-threshold concentrations through evaporation or cell metabolism, and MOR gene expression had returned to baseline levels. It would have been useful to measure ethanol concentrations over the duration of this experiment to determine how much the ethanol concentration fell due to evaporation or metabolism. However, MOR expression in cells treated under the 5 day refreshed treatment regimen was also no different from controls. Therefore, it appears ethanol treatment induces a transient decrease in MOR gene expression in SH-SY5Y cells.

\subsection{2 - Tobacco Extract Exposure increases MOR Gene Expression in SH-SY5Y}

The present study is the first to examine the effect of whole tobacco smoke extracts on the gene expression of MOR. Exposure to both HTPM and QTPM samples caused a significant upregulation in MOR gene expression in cells treated for three days and five days using the refreshed medium regimen (Fig. 59d). Since the QTPM sample was derived from denicotinized tobacco cigarettes, this would indicate that one or more unidentified non- 
nicotine components of cigarette smoke can induce up-regulation of MOR gene expression. However, under the refreshed medium treatment regimen nicotine exposure also induced a significant increase in MOR expression, although the increase was significantly smaller than the increase recorded in QTPM-exposed cells. These results indicate that chronic exposure to nicotine alone can cause up-regulation of MOR, and that MOR expression is also up-regulated by non-nicotine components of tobacco smoke. These increases in MOR expression were also found to correspond to greater amounts of MOR protein in SH-SY5Y cells, since Western blot analysis showed a statistically significant increase in MOR-specific staining in cells treated with nicotine, HTPM, and QTPM (Fig. 62c). These findings are unique to the present study, but are supported by evidence from earlier research.

Walters et al. (2005), for example examined the effect of chronic nicotine and cocaine exposure on MOR gene expression in wildtype mice. They reported that exposure to nicotine, but not cocaine led to a $40 \%$ increase in MOR mRNA expression in the ventral tegmental area, a response which was blocked in animals pretreated with the MOR antagonist naloxone. These findings show that MOR mRNA is up-regulated by chronic nicotine treatment, in agreement with the present study, and that activation of the $\mu$ receptor is required for receptor up-regulation. However, the present study also demonstrated that exposure to tobacco smoke extract from denicotinized Quest ${ }^{\circledR}$ cigarettes also caused an increase in MOR mRNA and protein expression, implying non-nicotine components of tobacco smoke also affect MOR gene expression.

The findings of Walters et al. (2005) and the present study demonstrate that nicotine and tobacco smoke induce a MOR response similar to that seen with cocaine. As discussed earlier, cocaine administration causes an increase in dopamine concentrations in the brain, and indirectly activates MOR through increasing concentrations of endogenous MOR agonists. Activation of MOR causes rapid internalization of the receptor, and gene expression is upregulated in response. Since nicotine is also known to increase extra-cellular dopamine concentrations in the brain, and the present study has confirmed that tobacco smoke extracts up-regulate MOR gene expression and protein abundance, it is possible that tobacco smoke indirectly activates MOR by a similar pathway as cocaine.

An alternative hypothesis is that the up-regulation of MOR reported in the present study may be due to epigenetic modification of the promoter region by tobacco extract, as proposed for MAO in section 6.4.2. The MOR proximal promoter contains $5 \mathrm{CpG}$ sites, and studies have shown demethylation of these sites increases MOR transcription (Hwang et al., 2007; Wei, 
2008). Hwang et al. (2007) examined the regulation of MOR expression in P19 pluripotent stem cells, which express MOR when differentiated by retinoic acid. They demonstrated that the methylation frequency of these $\mathrm{CpG}$ sites decreased as P19 differentiation proceded and MOR expression increased, and that treatment of undifferentiated cells with the DNA methylation inhibitor 5-aza-dC increased MOR expression. Therefore, it is possible that the tobacco smoke-induced increase in nucleic acid demethylase activity described by Launay et al. could be contributing to the up-regulation of MOR, as well as MAO. However, this hypothesis is highly speculative, and much more research is needed to clarify if tobacco smoke increases nucleic acid demethylase activity in SH-SY5Y cells, and the effects this may have on MOR gene expression.

Further investigation of this phenomenon is warranted to confirm the mechanisms by which tobacco smoke extract causes increased MOR expression, and to identify the non-nicotine compounds within tobacco smoke that influence MOR gene expression. It would also be interesting to examine whether nicotine and tobacco smoke extracts cause activation and rapid internalization of MOR, and induce the MOR signaling pathway in SH-SY5Y cells.

\subsection{3-MOR Up-Regulation Following Tobacco Exposure is Dependent on nAChR}

\section{Activation}

As discussed above, both nicotine and denicotinized cigarette smoke extract caused a significant up-regulation in MOR gene expression in SH-SY5Y cells. It is widely recognized that nicotine exerts many of its biochemical effects through activation of the nAChR, and that prolonged exposure to nicotine up-regulates nAChRs. Dunckley \& Lukas (2003) reported that nicotine exposure altered the expression of a range of genes in SH-SY5Y, and demonstrated that some of those changes in gene expression were inhibited or reversed when nicotine was administered with a nAchR antagonist (Dunckley \& Lukas, 2006). Their findings indicated that $\mathrm{nAChR}$ activation was required for nicotine to exert its effects on the genes examined in that study. While nicotine alone is known to up-regulate nAChRs, Ambrose et al. (2007) from our laboratory reported that TPM from whole tobacco smoke was more effective than nicotine alone at up-regulating nAChR expression. They concluded that tobacco smoke contained nonnicotine compounds that were also capable of increasing nAChR expression. The findings of 
these two studies were applied in the present experiment, to examine if $n A C h R$ activation is required for up-regulation of MOR.

When administered to cells alone, the nAChR antagonist mecamylamine significantly upregulated the expression of MOR (Fig. 61a). This is surprising, since mecamylamine is not known to directly interact with MOR. However, Dunckley \& Lukas (2006) also reported that mecamylamine altered the expression of several genes in SH-SY5Y, yielding effects similar to those of nicotine alone. They found that in almost every case where mecamylamine alone affected gene expression, mecamylamine also inhibited or reversed the effect of nicotine. This finding is also true of the present study, which showed that mecamylamine alone increased MOR gene expression in SH-SY5Y cells treated for 3 days, as did HTPM and QTPM samples (Fig. 61b). However, when mecamylamine was administered in conjunction with HTPM, MOR up-regulation was abolished, and when the antagonist was co-administered with QTPM, MOR up-regulation was reversed, resulting in down-regulation of the opioid receptor. This implies that the increased MOR gene expression due to HTPM or QTPM exposure observed in this study requires activation of nAChRs. However, what cannot be explained is why mecamylamine alone led to an increase in MOR mRNA expression. This finding implies that mecamylamine is inhibiting a basal level of endogenous cholinergic signalling in SH-SY5Y cells, and MOR resgulation is altered as a result, but it is uncertain whether these cells express this activity.

By up-regulating MOR, it appears that mecamylamine is mimicking the action of nicotine. This could occur if the signal to increase MOR transcription is triggered simply by occupation of the nAChR, by agonist or antagonist, without requiring receptor activation. However, in this case, mecamylamine and TPM would be presumed to have an additive effect on MOR expression when administered together, which was not observed. It is also possible that mecamylamine would mimic the action of TPM samples if their effect on MOR was due to blockade of the nAChR through receptor desensitization. However, these TPM samples are unlikely to cause complete desensitization of $\mathrm{nAChR}$ under the conditions tested here, as Sokolova et al. (2005) demonstrated that SH-SY5Y cells chronically treated with nicotine concentrations far exceeding those used here still retained some nAChR functioning. Furthermore, if MOR upregulation were the result of downstream events due to nAChR blockade, co-administration of TPM samples and mecamylamine would be expected to yield results similar to those of mecamylamine alone, since the concentration of mecamylamine used here is known to cause complete antagonism of nAChR function (Serres \& Carney, 2006). While it can't be ruled out 
that mecamylamine may cause MOR up-regulation by acting on MOR directly, or at another non-nAChR site this prospect is considered unlikely as no interactions of this nature have been reported in the literature.

It is interesting to note here that HTPM and QTPM both up-regulated MOR expression, but when these samples were co-administered with mecamylamine, MOR expression in HTPMtreated cells fell back to baseline, but in QTPM-treated cells MOR expression was significantly inhibited. While this result is difficult to interpret, it suggests that the chemical composition of QTPM is more considerably different to HTPM than just a lack of nicotine.

\section{$\underline{\text { 7.4.4-Summary }}$}

This study is the first to describe up-regulation of MOR expression in SH-SY5Y cells following chronic nicotine exposure, and also demonstrates MOR up-regulation following exposure to standard and denicotinized cigarette smoke extracts. This implies that both nicotine and nonnicotine compounds in tobacco smoke act to increase expression of MOR. Experiments with mecamylamine showed that antagonism of nAChRs either inhibited or reversed the observed up-regulation of MOR in HTPM and QTPM-treated cells, suggesting that nicotine, and nonnicotine components of tobacco smoke induced up-regulate MOR through a nAChRdependent mechanism. These findings further confirm involvement of the endogenous opioid system in the mechanisms of nicotine dependence, and offer new insights into the nature of these interactions, although further investigation is required to elucidate the mechanism of MOR up-regulation due to tobacco smoke. 


\section{Chapter Eight: General Discussion \& Future Directions}

\section{$\underline{8.1-\text { PROJECT SUMMARY }}$}

The overall aim of this project was to identify and characterize specific changes to cellular function as a result of exposure to tobacco smoke extracts. Of particular interest were those changes that could not be attributed to the action of nicotine. It is widely known that nicotine is the principle drug of dependence in tobacco, and a very extensive body of research has examined the neurobiological and physiological roles of nicotine in tobacco dependence. Nevertheless, the actions of nicotine alone cannot fully account for the intense and enduring nature of tobacco addiction. Previous research has provided strong evidence that monoamine oxidase enzyme inhibition and the endogenous opioid system play a role in the etiology and maintenance of tobacco dependence. The present study focused on examining changes in the activity and expression of these proteins in response to tobacco smoke extract exposure.

In agreement with previously published enzyme studies, tobacco smoke extracts collected from a wide range of tobacco products commercially available in New Zealand all inhibited the activity of purified human MAO-A and MAO-B enzymes. Extracts collected from denicotinized Quest ${ }^{\circledast}$ cigarettes also inhibited MAO-A and MAO-B in a manner similar to that of standard nicotine-containing cigarettes, effectively demonstrating that non-nicotine compounds within tobacco smoke are at least in part responsible for MAO inhibition. Different tobacco products vary considerably in their composition. This was evident in the present study, as significant differences were observed in the ability of different TPM samples to inhibit MAO-A and MAO-B. This implies there are significant variations in the concentration of MAO inhibitors in those tobacco products. These differences were evident between types of tobacco product (cigarettes versus loose-leaf tobacco), between different brands of cigarette, and even between different batches of the same brand. It has been previously established in rodent behavioural studies that co-administration of MAO inhibitors with nicotine greatly increases the intensity and persistence of addiction to nicotine (Guillem et al., 2005; A. S. 
Villegier et al., 2003; A.S. Villegier et al., 2007), and so it is possible that variations in MAO inhibitor action between different tobacco products may have a direct correlation to tobacco dependence in human smokers. Indeed, users of loose-leaf tobacco cigarettes score more highly on tests of nicotine dependence than habitual cigarette smokers (Young et al., 2006), and the present study found that extracts from loose-leaf tobacco products more potently inhibited MAO than manufactured cigarettes. Further research is required to examine this relationship in more depth, but if exposure to higher concentrations of MAO inhibitors is correlated with greater tobacco dependence in humans, this may make MAO inhibitor concentrations an important consideration for public health authorities and regulators of commercially available tobacco products.

Tobacco extract inhibition of MAO was much less clear-cut in experiments using cultured SH-SY5Y neuroblastoma cells, reflecting the usual differences in complexity between isolated enzyme preparations and whole cell systems. This study is the first to examine MAO inhibition due to whole tobacco smoke extracts in the SH-SY5Y cell line, and used TPM extract concentrations directly relevant to human smoking. Both standard tobacco TPM and denicotinized TPM significantly inhibited total MAO activity in these cells after 1-day short-term exposures, in line with previously published research demonstrating MAO inhibition following acute tobacco exposures. Also consistent with previously published data, HTPM extract induced a mixed-type inhibition, while QTPM inhibited MAO activity in a non-competitive manner. This suggests that while the denicotinized Quest ${ }^{\circledast}$ cigarettes inhibit MAO to the same degree as standard cigarettes, there are differences in the type and concentration of MAO inhibitors in the tobacco smoke. Interestingly, after 5 days exposure these tobacco extracts caused a considerable increase in total MAO activity in SH-SY5Y cells, a finding that has not been previously reported. The cause of this increase in activity is not known, but it was proposed that it could be due to increased concentrations of MAO-A and MAO-B protein in the cell as a compensatory mechanism in response to MAO inhibition. However, Western blot analysis failed to support this proposal. Investigation of MAO-A and MAO-B gene expression in these cells did support this theory, since mRNA expression of both genes was significantly up-regulated by both standard tobacco and denicotinized tobacco extracts. This indicates that MAO up-regulation is induced by the non-nicotine components of tobacco smoke, since mRNA expression was increased in response to the denicotinized QTPM sample, and nicotine alone did not alter expression of either MAO-A or MAO-B genes. This finding is unique to the present study, and further investigation is 
warranted to elucidate the mechanism of MAO gene up-regulation and the compound or compounds responsible. Additional gene expression experiments using mecamylamine found that this nAChR inhibitor effectively blocked the observed increases in MAO mRNA expression. This implies that although nicotine does not cause MAO up-regulation, activation of the nAChR is somehow involved in MAO gene expression. It also indicates that compounds within tobacco smoke other than nicotine are capable of activating the nAChR, or that SH-SY5Y cells possess endogenous nAChR agonist activity.

Further research is required to identify whether these changes in MAO gene expression are also observed in human smokers, and to establish their relevance to tobacco dependence. A recent study has reported that tobacco smoke causes an epigenetic modification of the MAO-B gene promoter, resulting in increased gene expression and protein concentration (Launay et al., 2009). If this is indeed the case, it could clarify some of the causes of nicotine withdrawal symptoms. If tobacco smoke up-regulates MAO expression as described, then while a smoker continues to receive regular doses of MAO inhibitors through cigarette smoking, any increased concentrations of MAO enzymes due to mRNA up-regulation will be inhibited. Upon quitting, inhibition of MAO activity is removed and these enzymes are free to degrade serotonin and dopamine levels in the brain. This mechanism could contribute to the depression and anhedonia experienced by many smokers in their attempts to quit. Avoidance of these withdrawal symptoms is an important factor in maintaining addiction, and is a common cause of relapse. This knowledge can be further applied to smoking cessation therapies to provide improved pharmacological support for quitting smokers. This may involve the inclusion of monoamine oxidase inhibitors with nicotine replacement therapies, a strategy that is already being trialed clinically (Berlin et al., 2002; Weinberger et al., 2010).

In addition to MAO, expression of the $\mu$ opioid receptor gene was also examined in response to chronic nicotine and tobacco smoke extract exposure. MOR has been widely studied with regard to mechanisms of drug dependence and addiction because of its crucial role in mediating the nociceptive and behavioural effects of morphine. Nicotine, HTPM, and QTPM treatment all increased MOR mRNA expression and MOR protein abundance. It has been proposed that nicotine may affect MOR indirectly by increasing extracellular dopamine. Dopamine overflow is thought to increase extracellular concentrations of endogenous MOR opioid, $\beta$-endorphin and the endomorphins, which in 
turn activate MOR and lead to rapid internalization of the $\mu$ receptor and a consequent up-regulation of MOR gene transcription. What is interesting is that denicotinized QTPM extract also increased MOR gene expression, indicating that tobacco compounds other than nicotine influence regulation of MOR. This finding is unique to the present study, and the mechanism of action has yet to be elucidated. Further investigation of the influence of tobacco smoke on MOR expression is required to determine if denicotinized tobacco smoke can increase dopamine, and therefore endogenous opioids. It remains to be determined whether tobacco smoke activates MOR, and what the non-nicotine compounds responsible for these effects are.

\section{2-FUTURE EXPERIMENTS}

\subsection{1 - Investigate a Continuous Cell Culture Treatment Regimen}

In the present study, investigations into the enzyme and gene expression responses of cultured cells to tobacco extracts demonstrated the considerable influence of cell culture conditions and treatment regimens in the results obtained. This was particularly evident when comparing cellular responses in cells treated for 5 days continuously, or with the refreshed treatment regimen. In order to eliminate the confounding effects of the culture conditions, future cell culture experiments could be performed using a continuous medium perfusion system, as described by Constantinescu et al. (2007). This method constantly replaces the cell culture medium with fresh medium, thus preventing the accumulation of cell metabolites which could perturb normal cell function. This is likely to be a more effective model of the in vivo environment in the CNS, and the cells are likely to respond to the treatment in a manner that more closely resembles the responses of neuronal cells in vivo. Furthermore, as Constantinescu et al. demonstrated, this culture technique can be successfully used to differentiate the SH-SY5Y cell line to express a phenotype more similar to dopaminergic neurons than to neuroblasts.

The use of a continuous perfusion cell culture system could also be extended to more accurately model tobacco exposure in human smokers. The present study treated cells with a single dose of tobacco smoke extract, but in human smoking the smoker is receives a dose of tobacco smoke with each cigarette smoked, and nicotine accumulates 
in the body during the day (N. L. Benowitz, 2008). This could be modeled by maintaining cells with a continuous medium perfusion system and dosing them directly and repeatedly with tobacco extracts using concentrations and exposure frequencies relevant to human smoking habits. It is possible that the MAO and MOR responses in cells cultured under continuous perfusion conditions will provide a better representation of human neuronal responses to tobacco smoke.

\subsection{2 - Identify the Neurobiologically Active Compounds in Tobacco Smoke}

The present study identified a number of intriguing and significant effects of tobacco smoke that could prove relevant to nicotine addiction. In order to more closely examine and effectively characterize these effects, the active compounds within tobacco smoke responsible for these cellular responses must be identified. This could be achieved by chemical fractionation of the TPM sample and adapting some of the experiments reported here for use as bio-assays to direct further investigation of the sample. For example, the MAO activity assay used in the present study has already been adapted for high-throughput experiments and could easily be used to identify TPM fractions that exhibit MAO inhibitory action. Active fractions could then be analyzed by gas chromatography and tandem mass spectrometry (GC-MS/MS) to identify compounds within the fraction. Candidate compounds can then be retested by bio-assay to confirm their bio-activity. Admittedly, identifying the tobacco compounds responsible for upregulation of the MAO and MOR gene will be difficult, as this approach is likely to be expensive and time consuming if qRT-PCR is used as a bio-assay. It is possible that novel MAO inhibitors may be identified in tobacco smoke, and these inhibitors may be useful in smoking cessation therapies, or perhaps other pharmaceutical applications, such as treatment of Parkinson's and Alzheimer's diseases.

\subsection{3 - Elucidate the Mechanisms of MAO Up-Regulation}

The present study reported up-regulation of MAO-A and MAO-B mRNA expression in response to tobacco smoke extracts, though the mechanism of this response was not elucidated. It is possible that the MAO gene up-regulation in SH-SY5Y cells observed in the present study is caused by demethylation of the core promoter region of MAO-A and 
MAO-B genes, as proposed by Launay, et al. (2009). To investigate this, detailed genetic analysis could be carried out to determine the methylation patterns of these genes in $\mathrm{SH}$ SY5Y cells treated chronically with tobacco smoke extract. This could involve bisulfate treatment of SH-SY5Y genomic DNA to convert all unmethylated cytosine nucleotides to uracil, followed by sequencing the MAO-A and MAO-B promoters. If the MAO-A and MAO-B promoter regions proved to have a reduced frequency of methylated $\mathrm{CpG}$ sites compared to untreated controls, as found by Launay et al., then the activity of nucleic acid demethylase could be measured to confirm this effect.

\subsection{4-Elucidate the Mechanisms of MOR Up-Regulation}

Nicotine is known to interact with MOR indirectly by increasing extracellular dopamine concentrations. It has been proposed that dopamine causes an increase in $\beta$-endorphin and endomorphin release, though this has yet to be adequately confirmed. To determine if nicotine and tobacco extracts interact with MOR through this mechanism, cellular concentrations of $\beta$-endorphin and the endomorphins could be measured using high performance liquid chromatography (HPLC) following acute and chronic treatment with the test compounds in cultured cells. Alternatively, this line of research could be extended to animal studies, by measuring changes in the extracellular concentrations of $\beta$-endorphin, endomorphin-1, and endomorphin-2 in key brain regions, including the nucleus accumbens and ventral tegmental area. This could be achieved through the use of microdialysis experiments and HPLC analysis.

\subsection{5 - Do Tobacco Smoke Constituents Bind to or Activate MOR?}

The present study reported that nicotine and other unidentified tobacco constituents upregulate MOR gene expression in SH-SY5Y cells. While it is possible that nicotine influences MOR expression indirectly through its ability to cause dopamine release, the mechanism of action of the denicotinized tobacco smoke extract should prove interesting. To help elucidate the mechanisms of MOR up-regulation it would be useful to identify whether any compounds in tobacco smoke bind directly to MOR or if they influence MOR indirectly. To determine if MOR activation is required for the gene upregulation described in the present study, the exposures and expression experiments could be repeated in the presence and absence of a MOR antagonist, such as naloxone. 
If MOR signaling is required for the observed up-regulation, this effect will be abolished in the presence of naloxone. This could also be tested through in vitro receptor autoradiography studies, to determine if tobacco smoke extracts can displace MOR-bound $\left[{ }^{3} \mathrm{H}\right]$-DAMGO. This assay has been widely reported in the literature and seems to produce good results. It would also be interesting to determine if tobacco extract or nicotine alone are capable of initiating the MOR signal transduction cascade. Since MOR is a Gprotein coupled receptor, activation can be investigated by measuring the binding of $\left[{ }^{35}\right.$ S]GTPYS to the active G-protein (Emmerson et al., 1996). This assay could be employed to test nicotine and tobacco extracts in cultured SH-SY5Y cells.

\subsection{6-Remodel in vitro Experiments for Animal Studies}

Cultured cells present a simple, relatively easily controlled, and convenient experimental system to test the behavior and function of individual cells. However, the brain is a complex network of heterogeneous inter-connected cells with different gene and protein expression profiles, and isolated cell studies are often woefully inadequate at modeling all of the complex interactions within the brain. While the results reported in the present study provide some insight into the response of neuronal cells to nicotine and tobacco extracts, these findings must be validated in vivo before a clearer understanding of the effects of tobacco smoke on human nicotine addiction can be gained.

Whole animal experiments should ideally involve investigations of MAO-A and -B enzyme activity, and the mRNA expression profiles of MAO and MOR genes in the brains of rodents taught to self administer tobacco extracts. The self-administration paradigm provides a more accurate model of human tobacco addiction, and has been used to study the effects of nicotine with some success (Corrigall et al., 2000). However, it may be difficult to train animals to self administer tobacco smoke extracts, especially denicotinized tobacco, since nicotine is the primary addictive agent in tobacco. The MAO activity assay presented in the present study could be used to assess MAO-A and MAO-B enzyme activity in platelets and dissected brain regions from self-administering rats, and a qRT-PCR assay could be utilized to assess MAO and MOR gene expression in the same brain samples. In particular, the expression of these genes should be examined in specific brain areas associated with drug addiction and dependence, such as the nucleus accumbens, ventral tegmental area, and striatum. Studies into the effects of whole tobacco smoke extract in animals will prepare the way for future validation and assessment in 
human smokers, and provide a framework for further development of smoking cessation therapies.

\section{3-TOWARDS IMPROVED SMOKING CESSATION THERAPIES}

Tobacco addiction is a major public health concern, and the development of effective smoking cessation strategies is necessary to help dependent smokers quit the habit. Nicotine induces addiction to smoking, through its complex neurobiological effects, affecting the activity of many neurotransmitter systems, including dopamine, serotonin, glutamate, and the endogenous opioid peptides. However, nicotine replacement therapies prove ineffective at maintaining abstinence in quitting smokers, suggesting a role for the non-nicotine components of tobacco smoke in maintaining addiction.

The experiments described in this thesis have identified several previously unreported effects of whole tobacco smoke on neuronal cellular function and behavior, and provide valuable insights into some of the complex cellular mechanisms influenced by tobacco smoke. These results demonstrate that tobacco compounds beyond nicotine have profound effects on the activity and expression of neural pathways associated with addiction, including monoamine oxidase activity and expression, and the endogenous opioid system. The research in the present study contributes to the growing body of knowledge of how tobacco dependence is established and maintained by the various components of tobacco smoke, and provides a framework for further validation and investigation of these systems in human smokers. Importantly, the present study has repeatedly demonstrated that the non-nicotine tobacco compounds in tobacco smoke have significant affects that may influence human tobacco dependence. This factor should be considered in future research into tobacco smoking, as examining the effects of nicotine alone are no longer sufficient to explain the many complex biochemical responses to tobacco smoke. Further research into the effects of the non-nicotine components of tobacco smoke is needed, and will complement existing knowledge on the pharmacology of nicotine. A thorough understanding of these complex cellular mechanisms is essential for the development of more effective therapeutic strategies for smoking cessation. 
- 205 - 


\section{References}

Abell, C. W., \& Kwan, S. W. (2001). Molecular characterization of monoamine oxidases A and B. Progress in Nucleic Acid Research and Molecular Biology, 65, 129-156.

Ambrose, V., Miller, J. H., Dickson, S. J., Hampton, S., Truman, P., Lea, R. A., et al. (2007). Tobacco particulate matter is more potent than nicotine at upregulating nicotinic receptors on SH-SY5Y cells. Nicotine \& Tobacco Research, 9(8), 793-799.

Azaryan, A. V., Clock, B. J., \& Cox, B. M. (1996a). Mu opioid receptor mRNA in nucleus accumbens is elevated following dopamine receptor activation. Neurochemical Research, 21(11), 1411-1415.

Azaryan, A. V., Coughlin, L. J., Buzas, B., Clock, B. J., \& Cox, B. M. (1996b). Effect of chronic cocaine treatment on mu- and delta-opioid receptor mRNA levels in dopaminergically innervated brain regions. Journal of Neurochemistry, 66(2), 443-448.

Bach, A. W., Lan, N. C., Johnson, D. L., Abell, C. W., Bembenek, M. E., Kwan, S. W., et al. (1988). cDNA cloning of human liver monoamine oxidase $A$ and $B$ : molecular basis of differences in enzymatic properties. Proceedings of the National Academy of Sciences of the USA, 85(13), 4934-4938.

Bagdy, G., \& Rihmer, Z. (1986). Measurement of platelet monoamine oxidase activity in healthy human volunteers. Acta physiologica Hungarica, 68(1), 19-24.

Balfour, D. J. (2002). The neurobiology of tobacco dependence: a commentary. Respiration, 69(1), 7-11.

Balfour, D. J., Bates, C., Benowitz, N., Berridge, V., Britton, J., Callum, C., et al. (2000a). Nicotine Addiction in Britain. London: Royal College of Physicians of London.

Balfour, D. J., \& Fagerstrom, K. O. (1996). Pharmacology of nicotine and its therapeutic use in smoking cessation and neurodegenerative disorders. Pharmacology \& Therapeutics, 72(1), 51-81.

Balfour, D. J., \& Ridley, D. L. (2000). The effects of nicotine on neural pathways implicated in depression: a factor in nicotine addiction? Pharmacology Biochemistry and Behavior, 66(1), 79-85.

Balfour, D. J., Wright, A. E., Benwell, M. E., \& Birrell, C. E. (2000b). The putative role of extrasynaptic mesolimbic dopamine in the neurobiology of nicotine dependence. Behavioral Brain Research, 113(1-2), 73-83.

Baron, J. A. (1986). Cigarette smoking and Parkinson's disease. Neurology, 36(11), 1490-1496.

Barrett, S. P., Boileau, I., Okker, J., Pihl, R. O., \& Dagher, A. (2004). The hedonic response to cigarette smoking is proportional to dopamine release in the human striatum as measured by positron emission tomography and [11C]raclopride. Synapse, 54(2), 6571.

Benowitz, N., Dains, K. M., Hall, S. M., Stewart, S., Wilson, M., Dempsey, D., et al. (2009). Progressive commercial cigarette yield reduction: biochemical exposure and behavioural assessment. Cancer epidemiology, biomarkers \& prevention: a publication of the American Association for Cancer Research cosponsored by the American Society of Preventative Oncology, 18(3), 876-883.

Benowitz, N. L. (2008). Clinical pharmacology of nicotine: implications for understanding, preventing, and treating tobacco addiction. Clinical Pharmacology \& Therapeutics, 83(4), 531-541.

Benwell, M. E., \& Balfour, D. J. (1992). The effects of acute and repeated nicotine treatment on nucleus accumbens dopamine and locomotor activity. British Journal of Pharmacology, 105(4), 849-856. 
Berlin, I., Aubin, H. J., Pedarroisse, A. M., Trames, A., Lancrenon, S., \& Lagrue, G. (2002). Lazabemide, a selective, reversible monoamine oxidase $B$ inhibitor, as an aid to smoking cessation. Addiction, 97(10), 1347-1354.

Berlin, I., Said, S., Spreux-Varoquaux, O., Launay, J. M., Olivares, R., Millet, V., et al. (1995a). A reversible monoamine oxidase $A$ inhibitor (moclobemide) facilitates smoking cessation and abstinence in heavy, dependent smokers. Clinical Pharmacology and Therapeutics, 58(4), 444-452.

Berlin, I., Said, S., Spreux-Varoquaux, O., Olivares, R., Launay, J. M., \& Puech, A. J. (1995b). Monoamine oxidase A and B activities in heavy smokers. Biological Psychiatry, 38(11), 756-761.

Berrendero, F., Kieffer, B. L., \& Maldonado, R. (2002). Attenuation of nicotine-induced antinociception, rewarding effects, and dependence in mu-opioid receptor knock-out mice. The Journal of Neuroscience, 22(24), 10935-10940.

Berrendero, F., Robledo, P., Trigo, J. M., Martin-Garcia, E., \& Maldonado, R. (2010). Neurobiological mechanisms involved in nicotine dependence and reward: participation of the endogenous opioid system. Neuroscience and Behavioural Reviews, 35, 220-231.

Berry, M. D., Juorio, A. V., \& Paterson, I. A. (1994). The functional role of monoamine oxidases $A$ and $B$ in the mammalian central nervous system. Progress in Neurobiology, 42(3), 375-391.

Betz, C., Mihalic, D., Pinto, M. E., \& Raffa, R. B. (2000). Could a common biochemical mechanism underlie addictions? Journal of Clinical Pharmacy and Therapeutics, 25, 11-20.

Biberman, R., Neumann, R., Katzir, I., \& Gerber, Y. (2003). A randomized controlled trial of oral selegiline plus nicotine skin patch compared with placebo plus nicotine skin patch for smoking cessation. Addiction, 98(10), 1403-1407.

Binda, C., Hubalek, F., Li, M., Edmondson, D. E., \& Mattevi, A. (2004). Crystal structure of human monoamine oxidase $B$, a drug target enzyme monotopically inserted into the mitochondrial outer membrane. FEBS Letters, 564(3), 225-228.

Binda, C., Newton-Vinson, P., Hubalek, F., Edmondson, D. E., \& Mattevi, A. (2002). Structure of human monoamine oxidase $B$, a drug target for the treatment of neurological disorders. Nature Structural Biology, 9(1), 22-26.

Boyadjieva, N. I., \& Sarkar, D. K. (1997). The secretory response of hypothalamic betaendorphin neurons to acute and chronic nicotine treatments and following nicotine withdrawal. Life Sciences, 61(6), 59-66.

Britt, J. P., \& McGehee, D. S. (2008). Presynaptic opioid and nicotinic receptor modulation of dopamine overflow in the nucleus accumbens. The Journal of Neuroscience, 28(7), 1672-1681.

Brodsky, M., Elliott, K., Hynansky, A., \& Inturrisi, C. E. (1995). CNS levels of mu opioid receptor (MOR-1) mRNA during chronic treatment with morphine or naltrexone. Brain Research Bulletin, 38(2), 135-141.

Brody, A. L., Olmstead, R. E., London, E. D., Farahi, J., Meyer, J. H., Grossman, P., et al. (2004). Smoking-induced ventral striatum dopamine release. The American Journal of Psychiatry, 161(7), 1211-1218.

Bullen, C., McRobbie, H., Thornley, S., Glover, M., Lin, R., \& Laugesen, M. (2010). Effect of an electronic nicotine delivery device (e cigarette) on desire to smoke and withdrawal, user preferences and nicotine delivery: randomised cross-over trial. Tobacco Control, 19, 98-103.

Bustin, S. A., Benes, V., Nolan, T., \& Pfaffl, M. W. (2005). Quantitative real-time RT-PCR - a perspective. Journal of Molecular Endocrinology, 34, 597-601. 
Cadoni, C., \& Di Chiara, G. (2000). Differential changes in accumbens shell and core dopamine in behavioral sensitization to nicotine. European Journal of Pharmacology, 387, R23R25.

Calafat, A. M., Polzin, G. M., Saylor, J., Richter, P., Ashley, D. L., \& Watson, C. H. (2004). Determination of tar, nicotine, and carbon monoxide yields in the mainstream smoke of selected international cigarettes. Tobacco Control, 13, 45-51.

Castagnoli, K., Steyn, S. J., Magnin, G., Van Der Schyf, C. J., Fourie, I., Khalil, A., et al. (2002). Studies on the interactions of tobacco leaf and tobacco smoke constituents and monoamine oxidase. Neurotoxicity Research, 4(2), 151-160.

Castelli, M. P., Melis, M., Mameli, M., Fadda, P., Diaz, G., \& Gessa, G. L. (1997). Chronic morphine and naltrexone fail to modify mu-opioid receptor mRNA levels in the rat brain. Molecular Brain Research, 45, 149-153.

Checkoway, H., Franklin, G. M., Costa-Mallen, P., Smith-Weller, T., Dilley, J., Swanson, P. D., et al. (1998). A genetic polymorphism of MAO-B modifies the association of cigarette smoking and Parkinson's disease. Neurology, 50(5), 1458-1461.

Chen, G., Yee, D. J., Gubernator, N. G., \& Sames, D. (2005). Design of optical switches as metabolic indicators: new fluorogenic probes for monoamine oxidases (MAO A and B). Journal of the American Chemical Society, 127, 4544-4545.

Chen, J., Higby, R., Tian, D., Tan, D., Johnson, M. D., Xiao, Y., et al. (2008). Toxicological analysis of low-nicotine and nicotine-free cigarettes. Toxicology, 249, 194-203.

Chen, K. (2004). Organization of MAO A and MAO B promoters and regulation of gene expression. Neurotoxicology, 25(1-2), 31-36.

Chen, Z. Y., Hotamisligil, G. S., Huang, J. K., Wen, L., Ezzeddine, D., Aydin-Muderrisoglu, N., et al. (1991). Structure of the human gene for monoamine oxidase type A. Nucleic Acids Research, 19(16), 4537-4541.

Christoffers, K. H., Li, H., Keenan, S. M., \& Howells, R. D. (2003). Purification and mass spectrometric analysis of the mu opioid receptor. Molecular Brain Research, 118, 119131.

Coccini, T., Castoldi, A. F., Gandini, C., Randine, G., Vittadini, G., Baiardi, P., et al. (2002). Platelet monoamine oxidase $B$ activity as a state marker for alcoholism: Trend over time during withdrawal and influence of smoking and gender. Alcohol and Alcoholism, 37(6), 566-572.

Constantinescu, R., Constantinescu, A. T., Reichmann, H., \& Janetzky, B. (2007). Neuronal differentiation and long-term culture of the human neuroblastoma line SH-SY5Y. Journal of Neural Transmission(Suppl 72), 17-28.

Contet, C., Kieffer, B. L., \& Befort, K. (2004). Mu opioid receptor: a gateway to drug addiction. Curr Opin Neurobiol, 14(3), 370-378.

Corrigall, W. A., Coen, K. M., Adamson, K. L., Chow, B. L. C., \& Zhang, J. (2000). Response of nicotine self-administration in the rat to manipulations of mu-opioid and gammaaminobutyric acid receptors in the ventral tegmental area. Psychopharmacology, 149, 107-114.

Costa-Mallen, P., Costa, L. G., \& Checkoway, H. (2005). Genotype combinations for monoamine oxidase-B intron 13 polymorphism and dopamine $D 2$ receptor TaqIB polymorphism are associated with ever-smoking status among men. Neuroscience Letters, 385(2), 158-162.

Counts, M. E., Hsu, F. S., Laffoon, S. W., Dwyer, R. W., \& Cox, R. H. (2004). Mainstream smoke constituent yields and predicting relationships from a worldwide market sample of cigarette brands: ISO smoking conditions. Regulatory Toxicology and Pharmacology, 39, 111-134.

Covey, L. S., Glassman, A. H., \& Stetner, F. (1997). Major depression following smoking cessation. American Journal of Psychiatry, 154(2), 263-265. 
D'Addario, C., ming, Y., Ogren, S. O., \& Terenius, L. (2008). The role of acetaldehyde in mediating effects of alcohol on expression of endogenous opioid system genes in a neuroblastoma cell line. The FASEB Journal, 22(3), 662-670.

Darrall, K. G., \& Figgins, J. A. (1998). Roll-your-own smoke yields: theoretical and practical aspects. Tobacco Control, 7, 168-175.

Davenport, K. E., Houdi, A. A., \& Van Loon, G. R. (1990). Nicotine protects against mu-opioid receptor antagonism by beta-funaltrexamine: evidence for nicotine-induced release of endogenous opioids in brain. Neuroscience Letters, 113(40-46).

Dhatt, R. K., Gudehithlu, K. P., Wemlinger, T. A., Tejwani, G. A., Neff, N. H., \& Hadjiconstantinou, M. (1995). Preproenkephalin mRNA and methionine-enkephalin content are increased in mouse striatum after treatment with nicotine. Journal of Neurochemistry, 64, 1878-1883.

Di Chiara, G., Bassareo, V., Fenu, S., De Luca, M. A., Spina, L., Cadoni, C., et al. (2004). Dopamine and drug addiction: the nucleus accumbens shell connection. Neuropharmacology, 47 Suppl 1, 227-241.

Di Chiara, G., \& Imperato, A. (1988). Drugs abused by humans preferentially increase synaptic dopamine concentrations in the mesolimbic system of freely moving rats. Proceedings of the National Academy of Sciences of the U S A, 85(14), 5274-5278.

Dunckley, T., \& Lukas, R. J. (2003). Nicotine modulates the expression of a diverse set of genes in the neuronal SH-SY5Y cell line. J Biol Chem, 278(18), 15633-15640.

Dunckley, T., \& Lukas, R. J. (2006). Nicotinic modulation of gene expression in SH-SY5Y neuroblastoma cells. Brain Research, 1116(1), 39-49.

Edmondson, D. E., Binda, C., \& Mattevi, A. (2004a). The FAD binding sites of human monoamine oxidases A and B. Neurotoxicology, 25(1-2), 63-72.

Edmondson, D. E., Binda, C., Wang, J., Upadhyay, A. K., \& Mattevi, A. (2009). Molecular and Mechanistic Properties of the Membrane-Bound Mitochondrial Monoamine Oxidases. Biochemistry, 48(20), 4220-4230.

Edmondson, D. E., Mattevi, A., Binda, C., Li, M., \& Hubalek, F. (2004b). Structure and mechanism of monoamine oxidase. Current Medicinal Chemistry, 11(15), 1983-1993.

Ekblom, J., Jossan, S. S., Bergstrom, M., Oreland, L., Walum, E., \& Aquilonius, S. M. (1993). Monoamine oxidase-B in astrocytes. Glia, 8(2), 122-132.

Ekblom, J., Zhu, Q. S., Chen, K., \& Shih, J. C. (1996). Monoamine oxidase gene transcription in human cell lines: treatment with psychoactive drugs and ethanol. Journal of Neural Transmission, 103(6), 681-692.

Elhwuegi, A. S. (2004). Central monoamines and their role in major depression. Progress in Neuropsychopharmacology \& Biological Psychiatry, 28(3), 435-451.

Emilsson, L., Saetre, P., Balciuniene, J., Castensson, A., Cairns, N., \& Jazin, E. (2002). Increased monoamine oxidase messenger RNA expression levels in frontal cortex of Alzheimer's disease patients. Neuroscience Letters, 326, 56-60.

Emmerson, P. J., Clark, M. J., Mansour, A., Akil, H., Woods, J. H., \& Medzihradsky, F. (1996). Characterization of opioid agonist efficacy in a $\mathrm{C}_{6}$ glioma cell line expressing the $\mu$ opioid receptor. The Journal of Pharmacology and Experimental Therapeutics, 278(3), 1121-1121.

Feindt, J., Becker, I., Blomer, U., Hugo, H. H., Mehdorn, H. M., Krisch, B., et al. (1995). Expression of somatostatin receptor subtypes in cultured astrocytes and gliomas. Journal of Neurochemistry, 65(5), 1997-2005.

Fichna, J., Janecka, A., Costentin, J., \& Do Rego, J.-C. (2007). The endomorphin system and its evolving neurophysiological role. Pharmacological Reviews, 59(1), 88-123.

Fowler, C. J., Mantle, T. J., \& Tipton, K. F. (1982). The nature of the inhibition of rat liver monoamine oxidase types $A$ and $B$ by the acetylenic inhibitors clorgyline, I-deprenyl and pargyline. Biochemical Pharmacology, 31(22), 3555-3561. 
Fowler, J. S., Logan, J., Wang, G. J., Volkow, N. D., Telang, F., Zhu, W., et al. (2003). Low monoamine oxidase $B$ in peripheral organs in smokers. Proceedings of the National Academy of Sciences of the U S A, 100(20), 11600-11605.

Fowler, J. S., Logan, J., Wang, G. J., Volkow, N. D., Telang, F., Zhu, W., et al. (2005). Comparison of monoamine oxidase $A$ in peripheral organs in nonsmokers and smokers. Journal of Nuclear Medicine, 46(9), 1414-1420.

Fowler, J. S., Volkow, N. D., Wang, G. J., Pappas, N., Logan, J., MacGregor, R., et al. (1996a). Inhibition of monoamine oxidase B in the brains of smokers. Nature, 379(6567), 733736.

Fowler, J. S., Volkow, N. D., Wang, G. J., Pappas, N., Logan, J., Shea, C., et al. (1996b). Brain monoamine oxidase A inhibition in cigarette smokers. Proceedings of the National Academy of Science of the U S A, 93(24), 14065-14069.

Fowler, J. S., Wang, G. J., Volkow, N. D., Franceschi, D., Logan, J., Pappas, N., et al. (2000). Maintenance of brain monoamine oxidase $B$ inhibition in smokers after overnight cigarette abstinence. American Journal of Psychiatry, 157(11), 1864-1866.

Fowles, J. (2003). Chemical composition of tobacco and cigarette smoke in two brands of New Zealand cigarettes. (Prepared as part of a New Zealand Ministry of Health contract for scientific services). Porirua, Wellington, NZ: Environmental Science and Research Ltd., from

http://www.ndp.govt.nz/moh.nsf/pagescm/1005/\$̧File/chemicalcompositioncigarette sbrands.pdf

Fowles, J., \& Dybing, E. (2003). Application of toxicological risk assessment principles to the chemical constituents of cigarette smoke. Tobacco Control, 12(4), 424-430.

George, T. P., \& O'Malley, S. S. (2004). Current pharmacological treatments for nicotine dependence. Trends in Pharmacological Sciences, 25(1), 42-48.

George, T. P., Vessicchio, J. C., Termine, A., Jatlow, P. I., Kosten, T. R., \& O'Malley, S. S. (2003). A preliminary placebo-controlled trial of selegiline hydrochloride for smoking cessation. Biological Psychiatry, 53(2), 136-143.

Gilbert, D. G., Meliska, C. J., Williams, C. L., \& Jensen, R. A. (1992). Subjective correlatesof cigarette-smoking-induced elevations of peripheral beta-endorphin and cortisol. Psychopharmacology (Berl), 106(2), 275-281.

Gilbert, D. G., Zuo, Y., Browning, R. A., Shaw, T. M., Rabinovich, N. E., Gilbert-Johnson, A. M., et al. (2003). Platelet monoamine oxidase $B$ activity changes across 31 days of smoking abstinence. Nicotine \& Tobacco Research, 5(6), 813-819.

Gotti, C., \& Clementi, F. (2004). Neuronal nicotinic receptors: from structure to pathology. Progress in Neurobiology, 74(6), 363-396.

Gourlay, S. G., \& Benowitz, N. (1997). Arteriovenous differences in plasma concentration of nicotine and catecholamines and related cardiovascular effects after smoking, nicotine nasal spray, and intravenous nicotine. Clinical Pharmacology and Therapeutics, 62, 453-463.

Gourlay, S. G., \& Benowitz, N. L. (1997). Arteriovenous differences in plasma concentration of nicotine and catecholamines and related cardiovascular effects after smoking, nicotine nasal spray, and intravenous nicotine. Clinical pharmacology and therapeutics, 62, 453-463.

Greenbaum, D., Jansen, R., \& Gerstein, M. (2002). Analysis of mRNA expression and protein abundance data: an approach for the comparison of the enrichment of features in the cellular population of proteins and transcripts. Bioinformatics, 18(4), 585-596.

Grimsby, J., Chen, K., Wang, L. J., Lan, N. C., \& Shih, J. C. (1991). Human monoamine oxidase A and $B$ genes exhibit identical exon-intron organization. Proceedings of the National Academy of Sciences of the U S A, 88(9), 3637-3641. 
Grimsby, J., Lan, N. C., Neve, R., Chen, K., \& Shih, J. C. (1990). Tissue distribution of human monoamine oxidase A and B mRNA. Journal of Neurochemistry, 55(4), 1166-1169.

Guang, H.-M., \& Du, G.-H. (2006). High-throughput screening for monoamine oxidase-A and monoamine oxidase-B inhibitors using one-step fluorescence assay. Acta Pharmacologica Sinica, 27(6), 760-766.

Guillem, K., Vouillac, C., Azar, M. R., Parsons, L. H., Koob, G. F., Cador, M., et al. (2005). Monoamine oxidase inhibition dramatically increases the motivation to selfadminister nicotine in rats. The Journal of Neuroscience, 25(38), 8593-8600.

Harro, J., Fischer, K., Vansteelandt, S., \& Harro, M. (2004). Both low and high activities of platelet monoamine oxidase increase the probability of becoming a smoker. European Neuropsychopharmacology, 14(1), 65-69.

Harro, M., Eensoo, D., Kiive, E., Merenakk, L., Alep, J., Oreland, L., et al. (2001). Platelet monoamine oxidase in health 9- and 15-year old children: the effect of gender, smoking and puberty. Progress in Neuro-psychopharmacology \& Biological Psychiatry, 25(8), 1497-1511.

Hauptmann, N., \& Shih, J. C. (2001). 2-Naphthylamine, a compound found in cigarette smoke, decreases both monoamine oxidase A and B catalytic activity. Life Sciences, 68(11), 1231-1241.

Heath, A. C., Kirk, K. M., Meyer, J. M., \& Martin, N. G. (1999). Genetic and social determinants of initiation and age at onset of smoking in Australian twins. Behavioral Genetics, 29(6), 395-407.

Herraiz, T., \& Chaparro, C. (2005). Human monoamine oxidase is inhibited by tobacco smoke: beta-carboline alkaloids act as potent and reversible inhibitors. Biochemical and Biophysical Research Communications, 326(2), 378-386.

Hoerndli, F. J., Toigo, M., Schild, A., Gotz, J., \& Day, P. J. (2004). Reference genes identified in SH-SY5Y cells using custom-made gene arrays with validation by quantitative polymerase chain reaction. Analytical Biochemistry, 335(1), 30-41.

Holschneider, D. P., \& Shih, J. C. (2000). Monoamine oxidase: Basic and clinical perspectives Psychopharmacology - The Fourth Generation of Progress: American College of Neuropsychopharmacology.

Horner, K. A., \& Zadina, J. E. (2004). Internalization and down-regulation of mu opioid receptors by endomorphins and morphine in SH-SY5Y human neuroblastoma cells. Brain Research, 1028, 121-132.

Houdi, A. A., Dasgupta, R., \& Kindy, M. S. (1998). Effect of nicotine use and withdrawal on brain preproenkephalin A mRNA. Brain Research, 799(2), 257-263.

Hwang, C. K., Song, K. Y., Kim, C. S., Choi, H. S., Guo, X.-H., Law, P.-Y., et al. (2007). Evidence of endogenous mu opioid receptor regulation by epigenetic control of the promoters. Molecular and Cellular Biology, 27(13), 4720-4736.

Inazu, M., Takeda, H., Ikoshi, H., Sugisawa, M., Uchida, Y., \& Matsumiya, T. (2001). Pjarmacological characterization and visualization of the glial serotonin transporter. Neurochemistry international, 39(1), 39-49.

Inazu, M., Takeda, H., \& Matsumiya, T. (2003). Expression and functional characterization of the extraneuronal monoamine transporter in normal human astrocytes. Journal of Neurochemistry, 84(1), 43-52.

Ino, M., Cole, G. M., \& Timiras, P. S. (1986). Tyrosine hydroxylase and monoamine oxidase-A activity increases in differentiating human neuroblastoma after elimination of dividing cells. Brain Research, 395(1), 120-123.

Ito, H., Hamajima, N., Matsuo, K., Okuma, K., Sato, S., Ueda, R., et al. (2003). Monoamine oxidase polymorphisms and smoking behaviour in Japanese. Pharmacogenetics, 13(2), 73-79. 
Jahng, J. W., Houpt, T. A., Wessel, T. C., Chen, K., Shih, J. C., \& Joh, T. H. (1997). Localization of monoamine oxidase $A$ and $B$ mRNA in the rat brain by in situ hybridization. Synapse, 25(1), 30-36.

Jarvik, M. E., Caskey, N. H., Wirshing, W. C., Madsen, D. C., Iwamoto-Schaap, P. N., Elins, J. L., et al. (2000). Bromocriptine reduces cigarette smoking. Addiction, 95(8), 1173-1183.

Karras, A., \& Kane, J. M. (1980). Naloxone reduces cigarette smoking. Life Sciences, 27, 15411545.

Keith, D. E., Anton, B., Murray, S. R., Zaki, P. A., Chu, P. C., Lissin, D. V., et al. (1997). $\mu$-opioid receptor internalization: opiate drugs have differential effects on a conserved endocytic mechanism in vitro and in the mammalian brain. Molecular Pharmacology, $53,377-384$.

Keith, D. E., Murray, S. R., Zaki, P. A., Chu, P. C., Lissin, D. V., Kang, L., et al. (1996). Morphine activates opioid receptors without causing their rapid internalization. The Journal of Biological Chemistry, 271(32), 19021-19024.

Khalil, A., Davies, B., \& Castagnoli, N., Jr. (2006). Isolation and characterization of a monoamine oxidase B selective inhibitor from tobacco smoke. Bioorganic \& Medicinal Chemistry, 14(10), 3392-3398.

Khalil, A., Steyn, S. J., \& Castagnoli, N. (2000). Isolation and Characterization of a Monoamine Oxidase Inhibitor from Tobacco Leaves. Chemical Research in Toxicology, 13, 31-35.

Khalil, A. A., Davies, B., \& Castagnoli, N. (2006). Isolation and characterization of a monoamine oxidase B selective inhibitor from tobacco smoke. Bioorganic \& Medicinal Chemistry, 14, 3392-3398.

Kieffer, B. L. (1999). Opioids: first lessons from knockout mice. Trends in Pharmacological Sciences, 20, 19-26.

Kivell, B. M., Day, D. J., McDonald, F. J., \& Miller, J. H. (2004). Developmental expression of $\mu$ and $\delta$ opioid receptors in the rat brainstem: evidence for a postnatal switch in $\mu$ isoform expression. Developmental Brain Research, 148, 185-196.

Knackstedt, L. A., LaRowe, S., Mardikian, P., Malcolm, R., Upadhyaya, H., Hedden, S., et al. (2009). The role of cystine-glutamate exchange in nicotine dependence in rats and humans. Biological Psychiatry, 65, 841-845.

Konradi, C., Kornhuber, J., Froelich, L., Fritze, J., Heinsen, H., Beckmann, H., et al. (1989). Demonstration of monoamine oxidase- $A$ and $-B$ in the human brainstem by a histochemical technique. Neuroscience, 33(2), 383-400.

Kraml, M. (1965). A rapid microfluorimetric determination of monoamine oxidase. Biochemical Pharmacology, 14, 1684-1686.

Krueger, M. J., \& Singer, T. P. (1993). An Examination of the Reliability of the Radiochemical Assay for Monoamine Oxidases A and B Analytical Biochemistry, 214, 116-123.

Laugesen, M. (2008). Safety report on the Ruyan e-cigarette cartridge and inhaled aerosol. Christchurch, New Zealand: Health New Zealand Ltd, from http://www.healthnz.co.nz/RuyanCartridgeReport30-Oct-08.pdf

Laugesen, M., Epton, M., Frampton, C. M. A., Glover, M., \& Lea, R. (2009). Hand-rolled cigarette smoking patterns compared with factory-made cigarette smoking in New Zealand men. BMC Public Health, 9(194).

Launay, J.-M., Del Pino, M., Chironi, G., Callebert, J., Peoc'h, K., Megnien, J.-L., et al. (2009). Smoking induces long-lasting effects through a monoamine-oxidase epigenetic regulation. PLOS ONE, 4(11).

Laviolette, S. R., \& van der Kooy, D. (2004). The neurobiology of nicotine addiction: bridging the gap from molecules to behaviour. Nature Reviews Neuroscience, 5(1), 55-65.

Law, P.-Y., Wong, Y. H., \& Loh, H. H. (2000). Molecular mechanisms and regulation of opioid receptor signalling. Annual Review of Pharmacology and Toxicology, 40, 389-430. 
Lawrence, D. M. P., Thomas, D. A., \& Wu, D.-Y. (2007). Glial Cells and the Neurobiology of Addiction. TheScientificWorldJOURNAL, 7(2), 86-88.

Lee, P. W., Wu, S., \& Lee, Y. M. (2004). Differential expression of mu-opioid receptor gene in CXBK and B6 mice by Sp1. Molecular Pharmacology, 66(6), 1580-1584.

Lena, I., Ombetta, J., Chalon, S., Dognon, A., Baulieu, J., Frangin, Y., et al. (1995). lododerivative of pargyline: a potential tracer for the exploration of monoamine oxidase sites by SPECT. Nuclear Medicine and Biology, 22(6), 727-736.

Levitt, P., Pintar, J. E., \& Breakefield, X. O. (1982). Immunocytochemical demonstration of monoamine oxidase $B$ in brain astrocytes and serotonergic neurons. Proceedings of the National Academy for Sciences of the U S A, 79(20), 6385-6389.

Livak, K. J., \& Schmittgen, T. D. (2001). Analysis of relative gene expression data using realtime quantitative PCR and the 2(-Delta Delta C(T)) Method. Methods, 25(4), 402-408.

Loh, H. H., \& Smith, A. P. (1990). Molecular characterization of opioid receptors. Annual Review of Pharmacology and Toxicology, 30, 123-147.

Lukas, R. J., Norman, S. A., \& Lucero, L. (1993). Characterization of nicotinic acetylcholine receptors expressed by cells of the SH-SY5Y human neuroblastoma clonal line. Molecular and Cellular Neurosciences, 4(1), 1-12.

Mahmood, I., Neau, S. H., \& Mason, W. D. (1994). An enzymatic assay for the MAO-B inhibitor selegiline in plasma. Journal of Pharmaceutical \& Buimedical Analysis, 12(7), 895-899.

Mansour, A., Fox, C. A., Akil, H., \& Watson, S. J. (1995). Opioid-receptor mRNA expression in the rat CNS: anatomical and functional implications. Trends in Neuroscience, 18(1), 2229.

Mansvelder, H. D., Keath, J. R., \& McGehee, D. S. (2002). Synaptic mechanisms underlie nicotine-induced excitability of brain reward areas. Neuron, 33(6), 905-919.

Marty, M. A., Erwin, V. G., Cornell, K., \& Zgombick, J. M. (1985). Effects of nicotine on betaendorphin, alpha MSH, and ACTH secretion by isolated perfused mouse brains and pituitary glands, in vitro. Pharmacol Biochem Behav, 22(2), 317 - 325.

Massey, J. B., \& Churchich, J. E. (1977). Kynuramine, a fluorescent substrate and probe of plasma amine oxidase. The Journal of Biological Chemistry, 252(22), 8081-8084.

Matsuzawa, S., Suzuki, T., \& Misawa, M. (2000). Involvement of mu-opioid receptor in the salsolinol-associated place preference in rats exposed to conditioned fear stress. Alcoholism: Clinical and Experimental Research, 24(3), 366-372.

Mazzio, E. A., Kolta, M. G., Reams, R. R., \& Soliman, K. F. (2005). Inhibitory effects of cigarette smoke on glial inducible nitric oxide synthase and lack of protective properties against oxidative neurotoxins in vitro. Neurotoxicology, 26(1), 49-62.

McConalogue, K., Grady, E. F., Minnis, J., Balestra, B., Tonini, M., Brecha, N. C., et al. (1999). Activation and internalization of the mu-opioid receptor by the newly disfcovered endogenous agonists, endomorphin-1 and endomorphin-2. Neuroscience, 90(3), 10511059.

McDonald, G. R., Hudson, A. L., Dunn, S. M. J., You, H., Baker, G. B., Whittal, R. M., et al. (2008). Bioactive contaminants leach from disposable laboratory plasticware. Science, $322,917$.

McGehee, D. S. (2006). Nicotinic and opioid receptor interactions in nicotine addiction. Mol Interv, 6(6), 311-314.

McGehee, D. S., Heath, M. J., Gelber, S., Devay, P., \& Role, L. W. (1995). Nicotine enhancement of fast excitatory synaptic transmission in CNS by pre-synaptic receptors. Science, 269, 1692-1696.

Mendez-Alvarez, E., Soto-Otero, R., Sanchez-Sellero, I., \& Lopez-Rivadulla Lamas, M. (1997a). Inhibition of brain monoamine oxidase by adducts of 1,2,3,4-tetrahydroisoquinoline with components of cigarette smoke. Life Sciences, 60(19), 1719-1727. 
Mendez-Alvarez, E., Soto-Otero, R., Sanchez-Sellero, I., \& Lopez-Rivadulla Lamas, M. (1997b). Inhibition of brain monoamine oxidase by adducts of 1,2,3,4-tetrahydroisoquinoline with components of cigarette smoke. Life Sci, 60(19), 1719-1727.

Meng, S. Z., Ozawa, Y., Itoh, M., \& Takashima, S. (1999). Developmental and age-related changes of dopamine transporter, and dopamine D1 and D2 receptors in human basal ganglia. Brain Research, 843(1-2), 136-144.

Minami, M., \& Satoh, M. (1995). Molecular biology of the opioid receptors: structures, functions and distributions. Neuroscience Research, 23, 121-145.

Mitoma, J., \& Ito, A. (1992). Mitochondrial targeting signal of rat liver monoamine oxidase B is located at its carboxy terminus. Journal of Biochemistry (Tokyo), 111(1), 20-24.

Morens, D. M., Grandinetti, A., Reed, D., White, L. R., \& Ross, G. W. (1995). Cigarette smoking and protection from Parkinson's disease: false association or etiologic clue? Neurology, 45(6), 1041-1051.

Morinan, A., \& Garratt, H. M. (1985). An improved fluorimetric assay for brain monoamine oxidase. J Pharmacol Methods, 13(3), 213-223.

Nagatsu, T. (2004). Progress in monoamine oxidase (MAO) research in relation to genetic engineering. Neurotoxicology, 25(1-2), 11-20.

Nie, L., Wu, G., \& Zhang, W. (2006). Correlation of mRNA expression and protein abundance affected by multiple sequence features related to translational efficiency in Desulfovibrio vulgaris: A quantitative analysis. Genetics, 174, 2229-2243.

O'Carroll, A. M., Fowler, C. J., Phillips, J. P., Tobbia, I., \& Tipton, K. F. (1983). The deamination of dopamine by human brain monoamine oxidase. Specificity for the two enzyme forms in seven brain regions. Naunyn Schmiedebergs Archives of Pharmacology, 322(3), 198-202.

O'Sullivan, J., Unzeta, M., Healy, J., O'Sullivan, M. I., Davey, G., \& Tipton, K. F. (2004). Semicarbazide-Sensitive Amine Oxidases: Enzymes with quite a lot to do. NeuroToxicology, 25, 303-315.

Okuyemi, K. S., Ahluwalia, J. S., \& Harris, K. J. (2000). Pharmacotherapy of smoking cessation. Archives of Family Medicine, 9(3), 270-281.

Olson, J. M., McNeel, W., Young, A. B., \& Mancini, W. R. (1992). Localization of the periphrealtype benzodiazepine binding site to mitochondria of human glioma cells. Journal of Neuro-oncology, 13(1), 35-42.

Ono, K., Toyono, T., \& Inenaga, K. (2008). Nicotinic receptor subtypes in rat subfornical organ neurons and glial cells. Neuroscience, 154(3), 994-1001.

Oreland, L. (1993). Monoamine oxidase in neuro-psychiatric disorders. In H. Yasuhara (Ed.), Monoamine oxidase (pp. 219-247).

Oreland, L., Fowler, C. J., \& Schalling, D. (1981). Low platelet monoamine oxidase activity in cigarette smokers. Life Sciences, 29(24), 2511-2518.

Pahlman, S., Hoehner, J. C., Nanberg, E., Hedborg, F., Fagerstrom, S., Gestblom, C., et al. (1995). Differentiation and survival influences of growth factors in human neuroblastoma. European Journal of Cancer, 31A(4), 453-458.

Pahlman, S., Mamaeva, S., Meyerson, G., Mattsson, M. E. K., Bjelfman, C., Ortoft, E., et al. (1990). Human neuroblastoma cells in culture: a model for neuronal cell differentiation and function. Acta physiologica Scandinavica. Supplementum., 592, 2537.

Paldyova, E., Bereczki, E., Santha, M., Wenger, T., Borsodi, A., \& Benyhe, S. (2007). Altered gene expression and functional activity of opioid receptors in the cerebellum of CB1 cannabinoid receptor knock out mice after acute treatments with cannabinoids. Acta Biologica Hungarica, 58 Suppl, 113-129. 
Pan, L., Xu, J., Yu, R., Xu, M.-M., Pan, Y.-X., \& Pasternak, G. W. (2005). Identification and characterization of six new alternatively spliced variants of the human mu opioid receptor gene, Oprm. Neuroscience, 133, 209-220.

Pare, C. M. B., \& Sandler, M. (1959). A clinical and biochemical study of a trial of Iproniazid in the treatment of depression. Journal of Neurology, Neurosurgery \& Psychiatry, 22(3), 247-251.

Patel, M. B., Patel, C. N., Rajashekara, V., \& Yoburn, B. C. (2002). Opioid agonists differentially regulate mu-opioid receptors and trafficking proteins in vivo. Molecular Pharmacology, 62(6), 1464-1470.

Pauly, J., Li, Q., \& Barry, M. B. (2007). Tobacco-free electronic cigarettes and cigars deliver nicotine and generate concern. Tobacco Control, 16(5), 357.

Peckys, D., \& Landwehrmeyer, G. B. (1998). Expression of mu, kappa, and delta opioid receptor messenger RNA in the human CNS: ${ }^{33} \mathrm{P}$ in situ hybridization study. Neuroscience, 88(4), 1093-1135.

Pelto-Huikko, M., Dagerlind, A., Kononen, J., Lundberg, J. M., Villar, M., Koistinaho, J., et al. (1995). Neuronal regulation of c-fos, c-jun, and junB immediate-early genes in rat adrenal medulla. The Journal of Neuroscience, 15(3 Pt 1), 1854-1868.

Peng, X., Gerzanich, V., Anand, R., Wang, F., \& Lindstrom, J. (1997). Chronic nicotine treatment up-regulates alpha3 and alpha7 acetylcholine receptor subtypes expressed by the human neuroblastoma cell line SH-SY5Y. Molecular Pharmacology, 51(5), 776-784.

Peters, I. R., Helps, C. R., Hall, E. J., \& Day, M. J. (2004). Real-time RT-PCR: considerations for efficient and sensitive assay design. Journal of Immunological Methods, 286, 203-217.

Pidoplichko, V. I., DeBiasi, M., Williams, J. T., \& Dani, J. A. (1997). Nicotine activates and densensitizes midbrain dopamine neurons. Nature, 390, 401-404.

Pidoplichko, V. I., Noguchi, J., Areola, O. O., Liang, Y., Peterson, J., Zhang, T., et al. (2004). Nicotinic cholinergic synaptic mechanisms in the ventral tegmental area contribute to nicotine addiction. Learning \& Memory, 11(1), 60-69.

Pinsonneault, J. K., Papp, A. C., \& Sadee, W. (2006). Allelic mRNA expression of X-linked monoamine oxidase a (MAOA) in human brain: dissection of epigenetic and genetic factors. Human Molecular Genetics, 15(17), 2636-2649.

Pomerleau, O. F., Fertig, J. B., Seyler, L. E., \& Jaffe, J. (1983a). Neuroendocrine reactivity to nicotine in smokers. Psychopharmacology (Berl), 81(1), 61-67.

Pomerleau, O. F., Fertig, J. B., Seyler, L. E., \& Jaffe, J. (1983b). Neuroendocrine reactivity to nicotine in smokers. Psychopharmacology, 81, 61-67.

Pontieri, F. E., Tanda, G., Orzi, F., \& Di Chiara, G. (1996). Effects of nicotine on the nucleus accumbens and similarity to those of addictive drugs. Nature, 382(6588), 255-257.

Quick, M. W., \& Lester, R. A. (2002). Desensitization of neuronal nicotinic receptors. Journal of Neurobiology, 53(4), 457-478.

Ramonet, D., Rodriguez, M., Saura, J., Lizcano, J. M., Romera, M., Unzeta, M., et al. (2003). Localization of monoamine oxidase $A$ and $B$ and semicarbazide-sensitive amine oxidase in human peripheral tissues. Inflammopharmacology, 11(2), 111-117.

Rao, T. S., Correa, L. D., Adams, P., Santori, E. M., \& Sacaan, A. I. (2003). Pharmacological characterization of dopamine, norepinephrine and serotonin release in the rat prefrontal cortex by neuronal nicotinic acetylcholine receptor agonists. Brain Research, 990(1-2), 203-208.

Richards, J. G., Saura, J., Ulrich, J., \& Da Prada, M. (1992). Molecular neuroanatomy of monoamine oxidases in human brainstem. Psychopharmacology (Berl), $106 \mathrm{Suppl,}$ S21-23.

Riederer, P., Danielczyk, W., \& Grunblatt, E. (2004). Monoamine Oxidase-B Inhibition in Alzheimer's Disease. Neurotoxicology, 25(1-2), 271. 
Rodriguez, M. J., Saura, J., Billett, E. E., Finch, C. C., \& Mahy, N. (2001). Cellular localization of monoamine oxidase $A$ and $B$ in human tissues outside of the central nervous system. Cell and Tissue Research, 304(2), 215-220.

Rommelspacher, H., Meier-Henco, M., Smolka, M., \& Kloft, C. (2002). The levels of norharman are high enough after smoking to affect monoamine oxidase $B$ in platelets. European Journal of Pharmacology, 441, 115-125.

Rose, J. E., Behm, F. M., Ramsey, C., \& Ritchie, J. C., Jr. (2001). Platelet monoamine oxidase, smoking cessation, and tobacco withdrawal symptoms. Nicotine \& Tobacco Research, 3(4), 383-390.

Rose, J. E., Behm, F. M., Westman, E. C., \& Coleman, R. E. (1999). Arterial nicotine kinetics during cigarette smoking and intravenous nicotine administration: implications for addiction. Drug and Alcohol Dependence, 56, 99-107.

Roth-Deri, I., Green-Sadan, T., \& Yadid, G. (2008). Beta-endorphin and drug-induced reward and reinforcement. Progress in Neurobiology, In press.

Sainz, A. (1960). Indications, Contraindications and Treatment with Monoamine Oxidase Inhibiting Antidepressant Drugs. Angiology, 11(94), 94-98.

Saland, L. C., Hastings, C. M., Abeyta, A., \& Chavez, J. B. (2005). Chronic ethanol modulates delta and mu-opioid receptor expression in rat CNS: immunohistochemical analysis with quantitative confocal microscopy. Neuroscience Letters, 381, 163-168.

Salminen, O., Murphy, K. L., McIntosh, J. M., Drago, J., Marks, M. J., Collins, A. C., et al. (2004). Subunit composition and pharmacology of two classes of striatal presynaptic nicotinic acetylcholine receptors mediating dopamine release in mice. Molecular Pharmacology, 65(6), 1526-1535.

Salokangas, R. K., Vilkman, H., Ilonen, T., Taiminen, T., Bergman, J., Haaparanta, M., et al. (2000). High levels of dopamine activity in the basal ganglia of cigarette smokers. The American Journal of Psychiatry, 157(4), 632-634.

Sandler, M., Reveley, M. A., \& Glover, V. (1981). Human platelet monoamine oxidase activity in health and disease: a review. Journal of Clinical Pathology, 34(3), 292-302.

Sano, M., Ernesto, C., Thomas, R. G., Klauber, M. R., Schafer, K., Grundman, M., et al. (1997). A controlled trial of selegiline, alpha-tocopherol, or both as treatment for Alzheimer's disease. The Alzheimer's Disease Cooperative Study. New England Journal of Medicine, 336(17), 1216-1222.

Satoh, M., \& Minami, M. (1995). Molecular pharmacology of the opioid receptors. [Review]. Pharmacology and therapeutics, 68(3), 343-364.

Saura, J., Bleuel, Z., Ulrich, J., Mendelowitsch, A., Chen, K., Shih, J. C., et al. (1996a). Molecular neuroanatomy of human monoamine oxidases $A$ and $B$ revealed by quantitative enzyme radioautography and in situ hybridization histochemistry. Neuroscience, 70(3), 755-774.

Saura, J., Kettler, R., Da Prada, M., \& Richards, J. G. (1992). Quantitative enzyme radioautography with $3 \mathrm{H}-\mathrm{Ro}$ 41-1049 and $3 \mathrm{H}-\mathrm{Ro}$ 19-6327 in vitro: localization and abundance of MAO-A and MAO-B in rat CNS, peripheral organs, and human brain. The Journal of Neuroscience, 12(5), 1977-1999.

Saura, J., Nadal, E., van den Berg, B., Vila, M., Bombi, J. A., \& Mahy, N. (1996b). Localization of monoamine oxidases in human peripheral tissues. Life Sciences, 59(16), 1341-1349.

Scherer, G. (1999). Smoking behaviour and compensation: a review of the literature. Psychopharmacology, 145, 1-20.

Schousboe, A., Sarup, A., Bak, L. K., Waagepetersen, H. S., \& Larsson, O. M. (2004). Role of astrocytic transport processes in glutamatergic and GABAergic neurotransmission. Neurochemistry international, 45(4), 521-527. 
Schroeder, J. A., Niculescu, M., \& Unterwald, E. M. (2003). Cocaine alters mu but not delta or kappa opioid receptor-stimulated in situ $\left[{ }^{35} \mathrm{~S}\right] \mathrm{GTP}$-deltaS binding in rat brain. Synapse, 47, 26-32.

Schurr, A., Porath, O., Krup, M., \& Livne, A. (1978). The effects of hashish components and their mode of action on monoamine oxidase from the brain. . Biochemical Pharmacology, 27(21), 2513-2517.

Schurr, A., \& Rigor, B. M. (1984). Cannabis extracts, but not delta 1-tetrahydrocannabinol, inhibits human brain and liver monoamine oxidase. General Pharmacology, 15(2), 171-174.

Serres, F., \& Carney, S. L. (2006). Nicotine regulates SH-SY5Y neuroblastoma cell proliferation through the release of brain-derived neurotrophic factor. Brain Research, 1101(1), 3642.

Sharma, G., \& Vijayaraghavan, S. (2002). Nicotinic receptor signaling in nonexcitable cells. Journal of Neurobiology, 53, 524-534.

Sherer, T. B., Trimmer, P., Borland, K., Parks, J. K., Bennett, J. P. J., \& Tuttle, J. B. (2001). Chronic reduction in complex I function alters calcium signalling in $\mathrm{SH}-\mathrm{SY} 5 \mathrm{Y}$ neuroblastoma cells. Brain Research, 891, 94-105.

Shih, J. C., Chen, K., \& Ridd, M. J. (1999). Monoamine oxidase: from genes to behavior. Annual Review of Neuroscience, 22, 197-217.

Simons, C. T., Cuellar, J. M., Moore, J. A., Pinkerton, K. E., Uyeminami, D., Carstens, M. I., et al. (2005). Nicotinic receptor involvement in antinociception induced by exposure to cigarette smoke. Neuroscience Letters, 389(2), 71-76.

Simpson, G. M., Shih, J. C., Chen, K., Flowers, C., Kumazawa, T., \& Spring, B. (1999). Schizophrenia, monoamine oxidase activity, and cigarette smoking. Neuropsychopharmacology, 20(4), 392-394.

Sivasubramaniam, S. D., Finch, C. C., Rodriguez, M. J., Mahy, N., \& Billett, E. E. (2003). A comparative study of the expression of monoamine oxidase-A and -B mRNA and protein in non-CNS human tissues. Cell and Tissue Research, 313(3), 291-300.

Snell, L. D., Glanz, J., \& Tabakoff, B. (2002). Relationships between effects of smoking, gender, and alcohol dependence on platelet monoamine oxidase-B: activity, affinity labeling, and protein measurements. Alcoholism: Clinical and Experimental Research, 26(7), 1105-1113.

Sokolova, E., Matteoni, C., \& Nistri, A. (2005). Desensitization of neuronal nicotinic receptors of human neuroblastoma SH-SY5Y cells during short or long exposure to nicotine. British Journal of Pharmacology, 146(8), 1087-1095.

Spanagel, R., Herz, A., \& Shippenberg, T. S. (1990). Identification of the opioid receptor types mediating beta-endorphin-induced alterations in dopamine release in the nucleus accumbens. European Journal of Pharmacology, 190, 177-190.

Stella, N. (2004). Cannabinoid signaling in glial cells. Glia, 48(4), 267-277.

Stephens, W. E. (2007). Dependence of tar, nicotine and carbon monoxide yields on physical parameters: implications for exposure, emissions control and monitoring. Tobacco Control, 16, 170-176.

Sweetman, A. J., \& Weetman, D. F. (1969). Polarographic assay of monoamine oxidase. British Journal of Pharmacology, 37(2), 550P.

Takeda, H., Inazu, M., \& Matsumiya, T. (2002). Astroglial dopamine transport is mediated by norepinephrine transporter. Naunyn Schmiedebergs Arch Pharmacol, 366(6), 620-623.

Talhout, R., Opperhuizen, A., \& van Amsterdam, J. G. (2007). Role of acetaldehyde in tobacco smoke addiction. European Neuropsychopharmacology, 17(10), 627-636.

Tan, E. K., Chai, A., Lum, S. Y., Shen, H., Tan, C., Teoh, M. L., et al. (2003). Monoamine oxidase B polymorphism, cigarette smoking and risk of Parkinson's disease: a study in an Asian 
population. American Journal of Medical Genetics Part B (Neuropsychiatric Genetics), 120(1), 58-62.

Tanda, G., \& Di Chiara, G. (1998). A dopamine-mu1 opioid link in the rat ventral tegmentum shared by palatable food (Fonzies) and non-psychostimulant drugs of abuse. European Journal of Neuroscience, 10(3), 1179-1187.

Teaktong, T., Graham, A. J., Johnson, M., Court, J. A., \& Perry, E. K. (2003). Selective changes in nicotinic acetylcholine receptor subtypes related to tobacco smoking: an immunohistochemical study. Neuropathology and Applied Neurobiology, 30, 243-254.

Teodorov, E., Modena, C. C., Sukikara, M. H., \& Felicio, L. F. (2006). Preliminary study of the effects of morphine treatment on opioid receptor gene expressionin brain structures of the female rat. Neuroscience, 141, 1225-1231.

Thorpe, L. W., Westlund, K. N., Kochersperger, L. M., Abell, C. W., \& Denney, R. M. (1987). Immunocytochemical localization of monoamine oxidases $A$ and $B$ in human peripheral tissues and brain. The Journal of Histochemistry and Cytochemistry, 35(1), 23-32.

Van Amsterdam, J. G., Talhout, R., Vleeming, W., \& Opperhuizen, A. (2006). Contribution of monoamine oxidase (MAO) inhibition to tobacco and alcohol addiction. [Minireview]. Life Sciences, 79, 1969-1973.

Vaughan, P. F. T., Kaye, D. F., Reeve, H. L., Ball, S. G., \& Peers, C. (1993). Nicotinic receptormediated release of noradrenaline in the human neuroblastoma SH-SY5Y. Journal of Neurochemistry, 60(6), 2159-2166.

Vaughan, P. F. T., Peers, C., \& Walker, J. H. (1995). The use of the human neuroblastoma SHSY5Y to study the effect of second messengers on noradrenaline release. [Review]. General Pharmacology, 26(6), 1191-1201.

Vecchiola, A., Collyer, P., Figueroa, R., Labarca, R., Bustos, G., \& Magendzo, K. (1999). Differential regulation of mu-opioid receptor mRNA in the nucleus accumbens shell and core accompanying amphetamine behavioural sensitization. Molecular Brain Research, 69(1), 1-9.

Vijayaraghavan, S. (2009). Glial-neuronal interactions - implications for plasticity and drug addiction. The AAPS Journal, 11(1), 123-132.

Villegier, A. S., Blanc, G., Glowinski, J., \& Tassin, J. P. (2003). Transient behavioral sensitization to nicotine becomes long-lasting with monoamine oxidases inhibitors. Pharmacology, Biochemistry and Behavior, 76(2), 267-274.

Villegier, A. S., Lotfipour, S., McQuown, S. C., Belluzzi, J. D., \& Leslie, F. M. (2007). Tranylcypromine enhancement of nicotine self-administration. Neuropharmacology, 52(6), 1415-1425.

Waldhoer, M., Bartlett, S. E., \& Whistler, J. L. (2004). Opioid Receptors. Annual Review of Biochemistry, 73, 953-990.

Walters, C. L., Cleck, J. N., Kuo, Y. C., \& Blendy, J. A. (2005). Mu-opioid receptor and CREB activation are required for nicotine reward. Neuron, 46(6), 933-943.

Watkins, L. R., Hutchinson, M. R., Johnston, I. N., \& Maier, S. F. (2005). Glia: novel counterregulators of opioid analgesia. Trends in Neurosciences, 28(12), 661-669.

Wei, L.-N. (2008). Epigenetic control of the expression of opioid receptor genes. Epigenetics, 3(3), 119-121.

Weinberger, A. H., Reutenauer, E. L., Jatlow, P. I., O'Malley, S. S., \& George, T. P. (2010). A double-blind, placebo-controlled, randomized clinical trial of oral selegiline hydrochloride for smoking cessation in nicotine-dependent cigarette smokers. Drug and Alcohol Dependence, 107(2-3), 188-195.

Westenberger, B. J. (2009). Evaluation of e-cigarettes. St Louis, Missouri, USA.: Food and Drug Administration, from http://www.fda.gov/downloads/Drugs/ScienceResearch/UCM173250.pdf 
Westlund, K. N., Denney, R. M., Kochersperger, L. M., Rose, R. M., \& Abell, C. W. (1985). Distinct monoamine oxidase A and B populations in primate brain. Science, 230(4722), 181-183.

Westlund, K. N., Denney, R. M., Rose, R. M., \& Abell, C. W. (1988). Localization of distinct monoamine oxidase $A$ and monoamine oxidase $B$ cell populations in human brainstem. Neuroscience, 25(2), 439-456.

Wewers, M. E., Dhatt, R. K., Snively, T. A., \& Tejwani, G. A. (1999). The effect of chronic administration of nicotine on antinociception, opioid receptor binding and metenkelphalin levels in rats. Brain Research, 822(1-2), 107-113.

Wewers, M. E., Dhatt, R. K., \& Tejwani, G. A. (1998). Naltrexone administration affects ad libitum smoking behaviour. Psychopharmacology, 140, 185-190.

Wewers, M. E., Tejwani, G. A., \& Anderson, J. (1994). Plasma nicotine, plasma beta-endorphin and mood states during periods of chronic smoking, abstinence and nicotine replacement. Psychopharmacology (Berl), 116(1), 98-102.

Weyler, W., Hsu, Y. P., \& Breakefield, X. O. (1990). Biochemistry and genetics of monoamine oxidase. Pharmacology \& Therapeutics, 47(3), 391-417.

WHO (2008). Marketers of electronic cigarettes should halt unproved therapy claims. Retrieved 27/01/2010, from http://www.who.int/mediacentre/news/releases/2008/pr34/en/index.html

Winkler, A., Buzas, B., Siems, W. E., Heder, G., \& Cox, B. M. (1998). Effect of ethanol drinking on the gene expression of opioid receptors, enkephalinase, and angiotensinconverting enzyme in two inbred mice strains. Alcoholism: Clinical and Experimental Research, 22(6), 1262-1271.

Wonnacott, S., Sidhpura, N., \& Balfour, D. J. (2005). Nicotine: from molecular mechanisms to behaviour. Current Opinion in Pharmacology, 5(1), 53-59.

Xue, Y., \& Domino, E. F. (2007). Tobacco/nicotine and endogenous brain opioids. Progress in Neuropsychopharmacology \& Biological Psychiatry.

Yabaluri, N., \& Medzihradsky, F. (1997). Down-regulation of mu-opioid receptor by full but not partial agonists is independent of G protein coupling. Molecular Pharmacology, 52, 896-902.

Yamada, M., \& Yasuhara, H. (2004). Clinical Pharmacology of MAO Inhibitors: Safety and Future. Neurotoxicology, 25(1-2), 215.

Yan, Z., Caldwell, G. W., Zhao, B., \& Reitz, A. B. (2004). A high-throughput monoamine oxidase inhibition assay using liquid chromatography with tandem mass spectrometry. Rapid Communications in Mass Spectrometry, 18, 834-840.

Youdim, M. B., \& Finberg, J. P. (1987). Monoamine oxidase B inhibition and the "cheese effect". Journal of Neural Transmission Supplement, 25, 27-33.

Young, D., Borland, R., Hammond, D., Cummings, K. M., Devlin, E., Yong, H.-H., et al. (2006). Prevalence and attributes of roll-your-own smokers in the International Tobacco Control (ITC) Four Country Survey. Tobacco Control, 15 (Suppl III).

Yu, P. H., \& Boulton, A. A. (1987). Irreversible inhibition of monoamine oxidase by some components of cigarette smoke. Life Sciences, 41(6), 675-682.

Yu, P. H., Davis, B. A., \& Boulton, A. A. (1992). Neuronal and astroglial monoamine oxidase: pharmacological implications of specific MAO-B inhibitors. Progress in Brain Research, 94, 309-315.

Yu, X., Mao, X., Blake, A. D., Li, W. X., \& Chang, S. L. (2003). Morphine and endomorphins differentially regulate mu-opioid receptor mRNA in SH-SY5Y human neuroblastoma cells. The Journal of Pharmacology and Experimental Therapeutics, ???

Yuan, J. S., Reed, A., Chen, F., \& Stewart, C. N., Jr. (2006). Statistical analysis of real-time PCR data. BMC Bioinformatics, 7, 85 . 
Yuan, J. S., Wang, D., \& Stewart, C. N., Jr. (2008). Statistical methods for efficiency adjusted real-time PCR quantification. Biotechnol Journal, 3(1), 112-123.

Zadina, J. E., Chang, S. L., Ge, L.-J., \& Kastin, A. J. (1993). Mu opiate receptor down-regulation by morphine and up-regulation by naloxone in SH-SY5Y human neuroblastoma cells. The Journal of Pharmacology and Experimental Therapeutics, 265(1), 254-262.

Zadina, J. E., Hackler, L., Ge, L.-J., \& Kastin, A. J. (1997). A potent and selective endogenous agonist for the mu-opiate receptor. Nature, 386, 499-502.

Zadina, J. E., Harrison, L. M., Ge, L.-J., Kastin, A. J., \& Chang, S. L. (1994). Differential regulation of mu and delta opiate receptors by morphine, selective agonists and antagonists and differentiating agents in SH-SY5Y human neuroblastoma cells. The Journal of Pharmacology and Experimental Therapeutics, 270(3), 1086-1096.

Zaki, P. A., Bilsky, E. J., Vanderah, T. W., Lai, J., Evans, C. J., \& Porreca, F. (1996). Opioid receptor types and subtypes: the delta receptor as a model. Annual Review of Pharmacology and Toxicology, 36, 379-401.

Zhou, M., \& Panchuk-Voloshina, N. (1997). A one-step fluorometric method for the continuous measurement of monoamine oxidase activity. Analytical Biochemistry, 253(2), 169174.

Zhu, Q. S., Grimsby, J., Chen, K., \& Shih, J. C. (1992). Promoter organization and activity of human monoamine oxidase (MAO) A and B genes. Journal of Neuroscience, 12(11), 4437-4446.

Zhuang, Z. P., Marks, B., \& McCauley, R. B. (1992). The insertion of monoamine oxidase A into the outer membrane of rat liver mitochondria. Journal of Biological Chemistry, 267(1), 591-596.

Zitova, A., Hynes, J., Kollar, J., Borisov, S. M., Klimant, I., \& Papkovsky, D. B. (2009). Analysis of activity and inhibition of oxygen-dependent enzymes by optical respirometry on the LightCycler system. Analytical Biochemistry, in press. 


\section{Chapter Nine: Appendix}

\section{$\underline{9.1-\text { BUFFERS \& SOLUTIONS }}$}

\section{Cell Lysis Buffer}

(1 mM MgCl2, 2mM EGTA, 1\% Nonidet P40, 50 mM Tris)

$250 \mu \mathrm{L} 2 \mathrm{M} \mathrm{MgCl} 2$

$0.38 \mathrm{~g}$ EGTA

$5 \mathrm{~mL} \quad$ Nonidet P40

$50 \mathrm{~mL} \quad 0.5 \mathrm{M}$ Tris-HCl, $\mathrm{pH} 6.8$

All reagents were dissolved in $300 \mathrm{~mL}$ of $\mathrm{dd}_{2} \mathrm{O}$, before the total volume was adjusted to 50 $\mathrm{mL}$ with additional $\mathrm{dd}_{2} \mathrm{O}$. This buffer was stored at $-20^{\circ} \mathrm{C}$ in $50 \mathrm{~mL}$ aliquots.

\section{0x Stock Phosphate Buffered Saline(PBS)}

$80.0 \mathrm{~g} \mathrm{NaCl}$

$2.0 \mathrm{~g} \mathrm{KCl}$

$26.8 \mathrm{~g} \mathrm{Na}_{2} \mathrm{HPO}_{4}$

All salts were dissolved in $800 \mathrm{~mL}$ of $\mathrm{ddH}_{2} \mathrm{O}$, and the solution $\mathrm{pH}$ was adjusted to $\mathrm{pH}=7.4$ with addition of $\mathrm{HCl}$ or $\mathrm{NaOH}$. Additional $\mathrm{ddH} 2 \mathrm{O}$ was added to make the solution up to 1 Litre. This stock was diluted to a $1 x$ solution before use. 


\section{Sodium Phosphate Buffer}

(50 mM Sodium phosphate, $150 \mathrm{mM}$ Sodium chloride)

$3.58 \mathrm{~g}$ Sodium Phosphate $\left(\mathrm{Na}_{2} \mathrm{HPO}_{4} \cdot 12 \mathrm{H}_{2} \mathrm{O}\right)$

$1.75 \mathrm{~g}$ Sodium chloride

Salts were dissolved in $100 \mathrm{~mL}$ of $\mathrm{ddH}_{2} \mathrm{O}$, and the $\mathrm{pH}$ of the solution was adjusted to $\mathrm{pH}=7.4$ with addition of concentrated $\mathrm{HCl}$ or $\mathrm{NaOH}$. Additional $\mathrm{ddH}_{2} \mathrm{O}$ was added to make the solution up to $200 \mathrm{~mL}$.

Tris Buffered Saline (TBS)

(50 mM Tris, $150 \mathrm{mM} \mathrm{NaCl)}$

$6.05 \mathrm{~g}$ Tris-base

$8.76 \mathrm{~g} \mathrm{NaCl}$

Salts were dissolved in $800 \mathrm{~mL}$ of $\mathrm{ddH} 2 \mathrm{O}$, and the $\mathrm{pH}$ was adjusted to $\mathrm{pH}=7.5$ with addition of $\mathrm{HCl}$. The solution was made up to a total 1 Litre volume with additional ddH2O.

\section{T-TBS}

$1 \mathrm{~mL}$ of Tween ${ }^{\circledR} 20$ was added to 1 Litre of TBS (see above).

\section{Western Transfer Buffer}

$9.09 \mathrm{~g}$ Tris-base

43.2 g Glycine

$3 \mathrm{~g} \quad$ Sodium diodecyl sulphate

$300 \mathrm{~mL}$ Methanol

All reagents were combined with ddH2O to a volume of 3 Litres. 
Agarose Gel (2\%)

$1 \mathrm{~g} \quad$ Agarose

$50 \mathrm{~mL}$ TAE Buffer

All reagents were combined in a glass beaker, and heated in a microwave for approximately 1 minute, until all agarose had dissolved. The solution was cooled briefly (approximately 1

minute), before addition of $10 \mu \mathrm{L}$ ethidium bromide solution. The gel solution was then poured smoothly into a agarose gel apparatus, and well forming combs were applied. Gels were run at $100 \mathrm{~V}$ for approximately 30 minutes.

DNA Loading Dye (6x)

$250 \mathrm{mg}$ Bromophenol Blue

$33 \mathrm{~mL} 150 \mathrm{mM}$ Tris, $\mathrm{pH} 7.6$

$60 \mathrm{~mL}$ Glycerol

$7 \mathrm{~mL} \quad \mathrm{ddH}_{2} \mathrm{O}$

Bromophenol blue was dissolved in Tris solution, before the addition of glycerol. The glycerol was mixed with the bromophenol solution thoroughly, and $\mathrm{ddH}_{2} \mathrm{O}$ was added to make the total volume $100 \mathrm{~mL}$.

\section{0x TAE Buffer}

$242 \mathrm{~g}$ Tris-base

$100 \mathrm{~mL} 0.5 \mathrm{M} \mathrm{Na}_{2} \mathrm{EDTA}(\mathrm{pH} 8.0)$

$57.1 \mathrm{mLGlacial}$ acetic acid

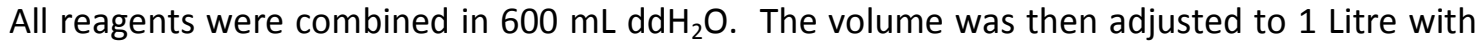
additional ddH2O. This stock was diluted to a $1 x$ solution with $\mathrm{ddH}_{2} \mathrm{O}$ before use. 


\section{2-TPM SAMPLE NUMBERING SCHEME}

The below numbering scheme was used to track TPM filters and the extracts produced from the in the laboratory. The data is reproduced here to elucidate sample abbreviations used in the text.

Table 21: TPM Sample numbering scheme and abbreviations

\begin{tabular}{lllll}
\hline & $\begin{array}{c}\text { Sample } \\
\text { Abbreviation }\end{array}$ & ESR Sample no. & Brand & Description \\
\hline Cigarettes & 15-Win & VA00015-20060317XEb & Winfield & King Size Filter \\
& 16-WinEM & VA00016-20060317XEb & Winfield & Extra Mild \\
& 17-B\&H & VA00017-20060317XEb & Benson \& Hedges & Special Filter \\
& 18-Pall & VA00018-20060317XEb & Pall Mall & Filter \\
& 19-Hol & VA00019-20060317XEb & Holiday & Special Filter \\
& 20-HolM & VA00020-20060317XEb & Holiday & Menthol Mild \\
& 21-Roth & VA00021-20060317XEb & Rothmans & King Size \\
35-Hol & VA00035-20070511XEb & Holiday & Regular \\
& 36-Hol & VA00036-20070511XEe & Holiday & Regular \\
& 32-Q3 & VA00032-20070511XEg & Quest 3 & Nicotine free \\
& 34-Q1 & VA00034-20070511XEg & Quest 1 & Low nicotine \\
& & & & \\
23-Port & VA00023-20060317XEb & Port Royal & \\
& 24-Park & VA00024-20060317XEb & Park Drive & \\
& 25-Drum & VA00025-20060317XEb & Drum & Menthol \\
& 26-Park & VA00026-20060317XEb & Park Drive & \\
\hline 27-HolTM & VA00027-20060317XEb & Holiday & Holiday & \\
\hline
\end{tabular}




\section{$\underline{9.3-\text { RUYAN }^{\circledR} \text { CARTRIDGE INGREDIENTS LIST }}$}

Reproduced from the RUYAN ${ }^{\circledR}$ Electronic Cigarette package insert:

Final Formulation:

Purified water

Alcohol

Tobacco Key Base \#1

Tobacco Key Base \#2

Nicotine

Propylene glycol

Tobacco Key Base \#1

Beta-Damascone

2,3-Diethylpyrazine

Michelia alba flower oil

Kentucky tobacco extract

Vanilla extract

5-methylfuran-2-one

Propylene glycol

Tobacco Key Base \#2

Benzyl carbonyl acetate

Ethyl tetra decanoate

Sweet fennel oil

Coffee flavouring base

Geranium oil

Leaf alcohol

Propylene glycol
$\%$

$$
\begin{gathered}
3.5-6 \\
4-6 \\
5 \\
0.8 \\
0.5-1.8 \\
80.4-86.2
\end{gathered}
$$




\section{4-PCR PRIMER EFFICIENCY DATA}

PCR efficiency of each primer pairs used in this study was tested as described in section 6.2.2.2. Briefly, $2 \mu \mathrm{g} / \mu \mathrm{L}$ total RNA from SH-SY5Y cells was serially diluted with $\mathrm{dH}_{2} \mathrm{O}$ over the range $1-256$ fold. Dilutions of cDNA were amplified in duplicate using relevant primer concentrations according to the protocol described in section 2.6 of this thesis. Mean $\mathrm{Ct}$ values for each dilution were plotted against the logarithm-base-10 transformed dilution factor, and linear regression analysis was used to fit a straight line to the data. The PCR

efficiency was calculated from the slope of this line using the equation: Efficiency $=10^{(-1 / \text { slope })}$ -1 .

Presented here are efficiency plots for all primer pairs used in this study.

(a)

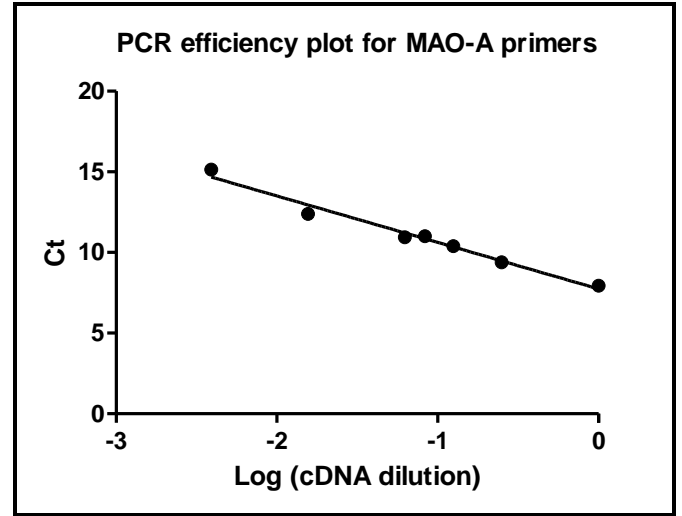

Figure 64a: Efficiency plot for MAO-A primer pairs. $y=-3.036 x+7.747, R^{2}=0.98$, Efficiency $=91.4 \%$. (b)

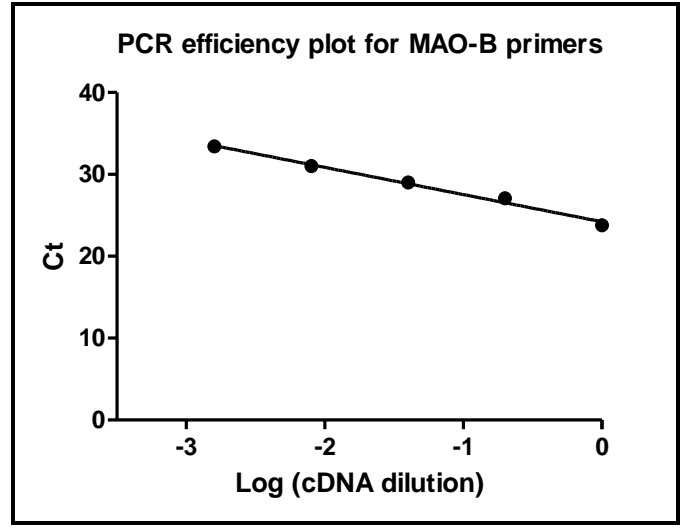

Figure 64b: Efficiency plot for MAO-B primer pairs. $y=-3.321 x+24.21, R^{2}=0.99$, Efficiency $=100 \%$. 
(c)

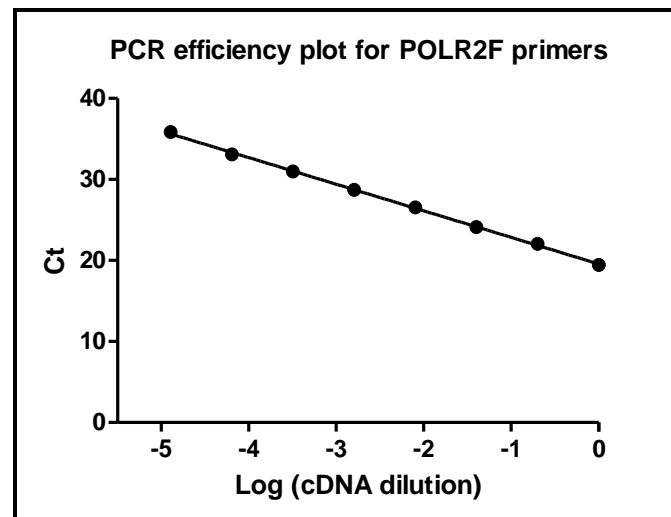

Figure 64c: Efficiency plot for POLR2F primer pairs. $y=-3.28 x+24.21, R^{2}=0.99$, Efficiency $=101.8 \%$.

(e)

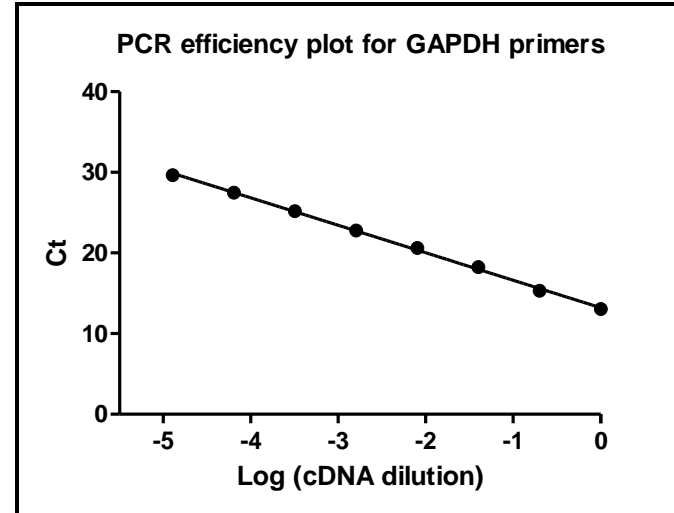

Figure 64e: Efficiency plot for GAPDH primer pairs. $y=-3.198 x+13.25, R^{2}=0.99$, Efficiency $=96.6 \%$.

(g)

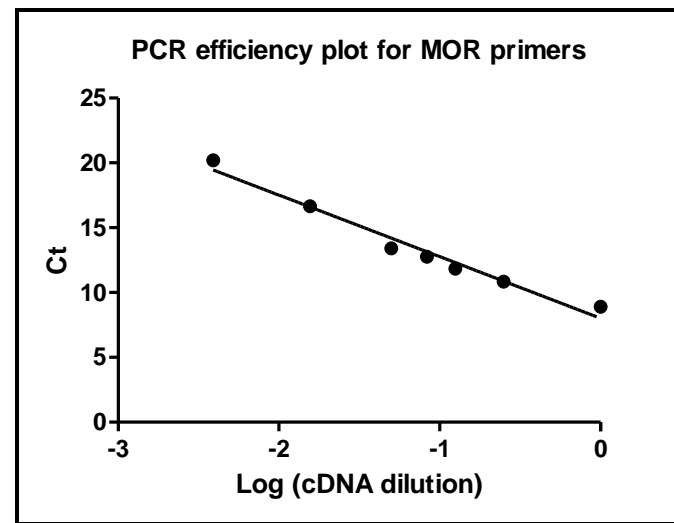

Figure 64g: Efficiency plot for MOR primer pairs. $y=-3.36 x+8.87, R^{2}=0.97$, Efficiency $=98.4 \%$. (d)

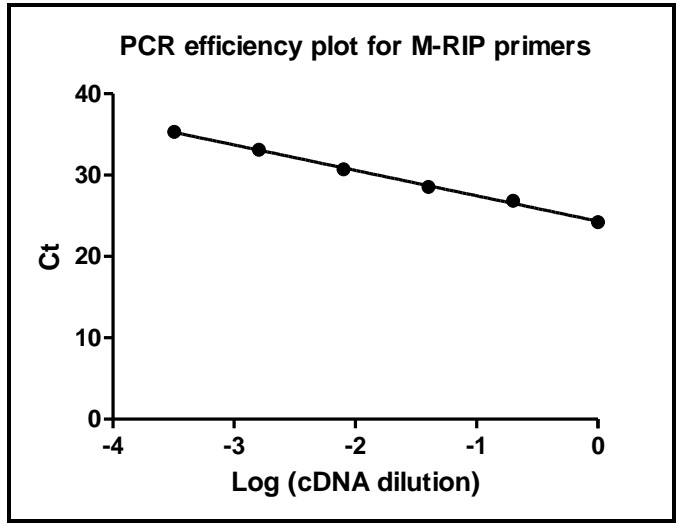

Figure 64d: Efficiency plot for M-RIP primer pairs. $y=-3.124 x+24.33, R^{2}=0.99$, Efficiency $=108 \%$.

(f)

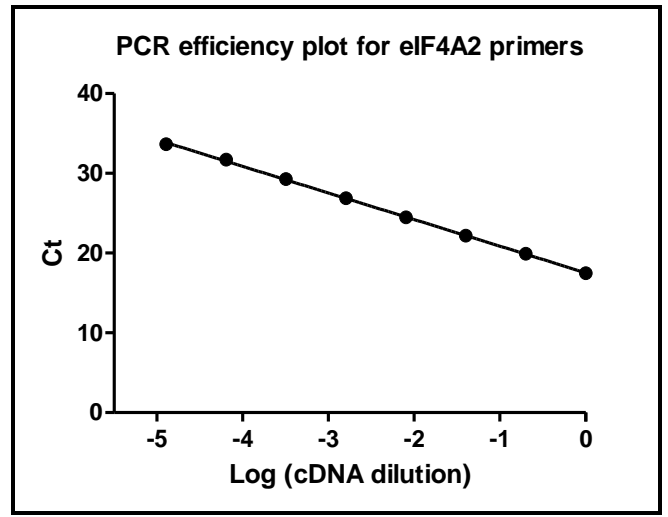

Figure 64f: Efficiency plot for elF4A2 primer pairs. $y=-3.335 x+17.53, R^{2}=0.99$, Efficiency $=99.5 \%$. 